\title{
Mineral dust induced pneumoconiosis: a pivotal role for the inflammasome
}

Citation for published version (APA):

Peeters, P. M. (2015). Mineral dust induced pneumoconiosis: a pivotal role for the inflammasome.

[Doctoral Thesis, Maastricht University]. Maastricht University. https://doi.org/10.26481/dis.20150529pp

Document status and date:

Published: 01/01/2015

DOI:

10.26481/dis.20150529pp

Document Version:

Publisher's PDF, also known as Version of record

\section{Please check the document version of this publication:}

- A submitted manuscript is the version of the article upon submission and before peer-review. There can be important differences between the submitted version and the official published version of record.

People interested in the research are advised to contact the author for the final version of the publication, or visit the DOI to the publisher's website.

- The final author version and the galley proof are versions of the publication after peer review.

- The final published version features the final layout of the paper including the volume, issue and page numbers.

Link to publication

\footnotetext{
General rights rights.

- You may freely distribute the URL identifying the publication in the public portal. please follow below link for the End User Agreement:

www.umlib.nl/taverne-license

Take down policy

If you believe that this document breaches copyright please contact us at:

repository@maastrichtuniversity.nl

providing details and we will investigate your claim.
}

Copyright and moral rights for the publications made accessible in the public portal are retained by the authors and/or other copyright owners and it is a condition of accessing publications that users recognise and abide by the legal requirements associated with these

- Users may download and print one copy of any publication from the public portal for the purpose of private study or research.

- You may not further distribute the material or use it for any profit-making activity or commercial gain

If the publication is distributed under the terms of Article $25 \mathrm{fa}$ of the Dutch Copyright Act, indicated by the "Taverne" license above, 


\section{MINERAL DUST INDUCED PNEUMOCONIOSIS: A PIVOTAL ROLE FOR THE INFLAMMASOME}


(C) 2015 Paul Peeters, Hasselt, België

All rights reserved. No part of this book may be reproduced or transmitted, in any form or by any means, without written permission from the author.

Production: Datawyse | Universitaire Pers Maastricht

ISBN: 978-94-6159-442-6

\section{Oltín \\ intintit}

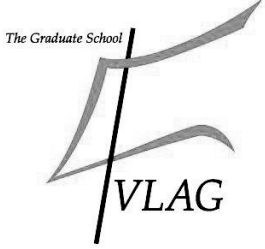

The studies presented in this thesis were performed at the Nutrition and Toxicology Research Institute Maastricht (NUTRIM), which participates in the Graduate School VLAG (Food Technology, Agrobiotechnology, Nutrition, and Health Sciences), accredited by the Royal Netherlands Academy of Arts and Sciences (KNAW).

Maastricht UMC+

az 


\title{
MINERAL DUST INDUCED PNEUMOCONIOSIS: A PIVOTAL ROLE FOR THE INFLAMMASOME
}

\author{
Dissertation \\ To obtain the degree of doctor at Maastricht University \\ on the authority of the Rector Magnificus, Prof. dr. L.L.G. Soete \\ in accordance with the decision of the Board of Deans, \\ to be defended in public on Friday May $29^{\text {th }} 2015$ at 10.00 am \\ by
}

Paul Michel Peeters 


\section{Promotors:}

Prof. dr. E.F.M. Wouters

Prof. dr. B.T. Mossman, University of Vermont, USA

\section{Copromotor:}

Dr. N.L. Reynaert

\section{Beoordelingscommissie:}

Prof.dr B. Kramer, voorzitter/chairman

Prof.dr. A. Bast

Prof.dr. B. Nemery, KU Leuven, BE

Prof.dr. P. De Vuyst, Erasmus Hospital Brussel, BE

Dr. R. Sverdlov

The studies described in this thesis were supported by The Weijerhorst Foundation. 
"I do not believe in a personal God and I have never denied this but have expressed it clearly. If something is in me which can be called religious then it is the unbounded admiration for the structure of the world so far as our science can reveal it."

Albert Einstein 



\section{TABLE OF CONTENTS}

Table of contents

List of abbreviations

9

Chapter 1 General introduction and thesis outline

Chapter 2 Pathogenesis and mechanisms of asbestos and silicosis

Chapter 3 Indications for distinct pathogenic mechanisms of asbestos and silica through gene expression profiling of the response of lung epithelial cells

Chapter 4 Differences in gene expression and cytokine production by crystalline vs. amorphous silica in human lung epithelial cells

Chapter 5 Immune homeostasis in epithelial cells: evidence and role of inflammasome signaling reviewed

Chapter 6 Silica induces NLRP3 inflammasome activation in human lung epithelial cells

Chapter 7 Silica-induced NLRP3 inflammasome activation in vitro and in rat lungs

Chapter 8 Inflammasome activation in human silicosis

Chapter 9 Discussion and future directions

Chapter 10 Summary/Samenvatting

Appendices Valorisation paragraph

Dankwoord/acknowledgements

List of publications

Curriculum vitae 



\begin{tabular}{|c|c|}
\hline AP-1 & Activator protein-1 \\
\hline $\mathrm{AEC}$ & Alveolar epithelial cell \\
\hline AIM2 & Absent in melanoma 2 \\
\hline ALR & AIM2-like receptor \\
\hline AM & Alveolar macrophage \\
\hline ASC & Apoptosis-associated speck-like protein containing a CARD \\
\hline ATP & Adenosine-5'-triphosphate \\
\hline BALF & Bronchoalveolar lavage fluid \\
\hline bFGF & basic fibroblast growth factor \\
\hline CARD & Caspase recruitment domain \\
\hline CINC & Cytokine-induced neutrophil chemoattractant \\
\hline $\mathrm{COX}-2$ & Cyclooxygenase-2 \\
\hline DAMPs & Danger-associated molecular patterns \\
\hline Der p1 & Dermatophagoides pteronyssinus 1 \\
\hline DNA & Deoxyribonucleic acid \\
\hline dsDNA & Double-stranded deoxyribonucleic acid \\
\hline DSS & Dextran sodium sulphate \\
\hline ECM & Extracellular matrix \\
\hline EGF & Epidermal growth factor \\
\hline EGR & Early growth response \\
\hline ELISA & Enzyme-linked immunosorbent assay \\
\hline ERK & Extracellular signal regulated kinase \\
\hline FGF & Fibroblast growth factor \\
\hline GPX & Glutathione peroxidase \\
\hline GSH & Glutathione \\
\hline HIN-200 & 200 amino acid hemopoietic IFN- inducible nuclear proteins \\
\hline HMGB1 & High mobility group box protein 1 \\
\hline HO-1 & Heme oxygenase-1 \\
\hline HPV & Human papillomavirus \\
\hline IBD & Irritable bowel disease \\
\hline ICE & Interleukin 1 converting enzyme \\
\hline IEC & Intestinal epithelial cell \\
\hline IFI16 & Interferon alpha-inducible protein 16 \\
\hline IFIX & Interferon inducible protein $\mathrm{X}$ \\
\hline IFN & Interferon \\
\hline IGF & Insulin-like growth factor \\
\hline IL & Interleukin \\
\hline IL-18 & Interleukin 18 \\
\hline IL-1R & Interleukin 1 receptor \\
\hline $\mathrm{IL}-1 \alpha$ & Interleukin $1 \alpha$ \\
\hline IL-1 $\beta$ & Interleukin $1 \beta$ \\
\hline ІкB & I kappa B kinase \\
\hline JNK & c-jun N-terminal kinase \\
\hline
\end{tabular}




\begin{tabular}{|c|c|}
\hline LPS & Lipopolysaccharide \\
\hline LRR & Leucine-rich repeat domain \\
\hline MAC & Membrane attack complex \\
\hline MAPK & Mitogen-activated protein kinase \\
\hline MARCO & Macrophage receptor with collagenous structure \\
\hline MCP-1 & Monocyte chemotactic protein-1 \\
\hline MIP & Macrophage inflammatory protein \\
\hline MMP & Matrix metalloproteases \\
\hline MNDA & Myeloid nuclear differentiation antigen \\
\hline MSU & Monosodium Urate \\
\hline MTB & Mycobacterium tuberculosis \\
\hline MWCNT & Multi-walled carbon nanotubes \\
\hline MyD88 & Myeloid differentiation primary response protein \\
\hline NACHT & Nucleotide-binding and oligomerization domain \\
\hline NADPH & Nicotinamide adenine dinucleotide phosphate \\
\hline $\mathrm{NADPH}(\mathrm{O})$ & Nicotinamide adenine dinucleotide phosphate-(oxidase) \\
\hline NAIP & NLR family, apoptosis inhibitory protein \\
\hline $\mathrm{NF} \kappa \mathrm{B}$ & Nuclear factor kappa B \\
\hline NK cells & Natural killer cells \\
\hline NLR & Nucleotide-binding domain leucine-rich repeat containing receptors \\
\hline NLRC4 & NLR with a CARD domain 4 \\
\hline NLRC5 & NLR with a CARD domain 5 \\
\hline NLRP1 & NLR with a pyrin domain 1 \\
\hline NLRP10 & NLR with a pyrin domain 10 \\
\hline NLRP12 & NLR with a pyrin domain 12 \\
\hline NLRP3 & NLR with a pyrin domain 3 \\
\hline NLRP6 & NLR with a pyrin domain 6 \\
\hline NLRP7 & NLR with a pyrin domain 7 \\
\hline NOD & Nucleotide-binding oligomerization containing domain \\
\hline P2X7 & Purinergic receptor P2X, ligand-gated ion channel, 7 \\
\hline PAMPs & Pathogen-associated molecular patterns \\
\hline PDGF & Platelet-derived growth factor \\
\hline PGE & Prostaglandin E \\
\hline PKC & Protein kinase $\mathrm{C}$ \\
\hline PMF & Progressive massive fibrosis \\
\hline PRR & Pattern recognition receptor \\
\hline PRX & Peroxiredoxin \\
\hline PYD & Pyrin domain \\
\hline PYHIN & Pyrin and HIN200 domain containing protein \\
\hline RAGE & Receptor for advanced glycation end-product \\
\hline RNA & Ribonucleic acid \\
\hline ROS/RNS & Reactive oxygen and nitrogen species \\
\hline RSV & Respiratory syncytial virus \\
\hline
\end{tabular}


$\mathrm{SiO} 2 \quad$ Silicon

siRNA Small interfering RNA

SOD2 Superoxide dismutase 2

SR-AI Scavenger receptor AI

TGF- $\beta \quad$ Transforming growth factor $\beta$

TIMP Tissue inhibitor of matrix metalloproteinases

TiO2 Titanium oxide

TNBSA 2,4,6-trinitrobenzene sulfonic acid

TNF- $\alpha \quad$ Tumor necrosis factor $\alpha$

WCL Whole cell lysate 



\section{CHAPTER 1}

GENERAL INTRODUCTION AND THESIS OUTLINE 



\section{GENERAL INTRODUCTION}

Inhalation is a vital process for all human life. By definition, inhalation is an active or voluntary drawing of air (and its contents) from the external environment, through the airways, and into the alveoli via nose or mouth. Since environmental air is mostly unfiltered, inhalation of these other contents can swing back with unfavorable consequences. The variety and range of pulmonary and systemic abnormalities that environmental exposure by inhalation of substances such as cigarette smoke, air pollutants and allergens but also asbestos and silica can cause, is vast. Pneumoconiosis refers to a range of diseases that are caused by the inhalation of organic and non-organic dusts which are then retained in the lung. Documentation on exposure to mineral dust particles from occupational and environmental sources and their associations with pulmonary abnormalities, including fibrotic lung disease, is present since the $1500 \mathrm{~s}$. The resultant severity of pulmonary disorders depends on the susceptibility of lungs; size, concentration, solubility and fibrogenic properties of the inhaled particles; and duration of exposure. Inflammatory signaling is an overlapping hallmark in the developmental process of asbestos and silica-induced diseases. Upon inhalation, toxic particulates initially interact with the epithelial cells lining the respiratory tract and with resident macrophages. Upon interaction and engulfment of particles macrophages are stimulated and accompanied with the activation of intracellular danger sensors, such as the inflammasome, many proinflammatory mediators, fibrogenic factors, reactive oxygen and nitrogen species are released. This has paracrine effects on the epithelium and promotes further inflammatory cell recruitment as well as fibroblast proliferation. Generally, it is believed that macrophages are the critical mediators in the onset and fueling of the inflammatory processes although in recent years a much larger role has been attributed to the epithelium as being far less passive in its contributions to the inflammatory cascade and the disease development. Evidence is now suggesting that epithelial cells are important barrier building blocks that actively cooperate with neighboring cells to mediate innate inflammatory responses upon dust exposures. Activated molecular patterns upon contact of epithelium with silica and asbestos as well as the characterization and understanding of the epithelial cell as a danger sensor in vitro and in vivo are the general concepts that will be investigated in this thesis. 


\section{THESIS OUTLINE}

Both types of pneumoconiosis, asbestosis and silicosis, eventually drastically diverge in the pathological presentation or phenotypical outcome, regardless of the prolonged chronic inflammation induced by both minerals. The objective to create a state of the art review with respect to definition, history, physicochemical background and presentation as well as possible molecular explanations on the pathogenesis is assessed in Chapter 2. However, the exact reason why these two interstitial lung diseases show such a distinct histopathological phenotype and if there is a role for the epithelium in this specifically altered disturbed defense mechanism remains largely unknown.

Therefore, the aim was to demonstrate if early and intrinsic divergent molecular mechanisms in epithelial cells could lead to distinct aspects of proliferation and cancer development by asbestos fibers. And on the other hand, if chronic inflammatory signaling pathways induced by silica, could be predominantly associated with remodeling and fibrosis. In Chapter $\mathbf{3}$ gene profiling was used to examine commonalities and differences in response of normal human bronchial epithelial cells to crocidolite asbestos or cristobalite silica in vitro.

In Chapter $\mathbf{4}$ it was determined if the degree and magnitude of these responses in vitro could be predictive in determining the pathogenicity associated with the crystallinity of potentially harmful particulates. Here, robust microarray profiling was utilized to assess the extent of changes in gene expression followed by comparing responses cytokine production in human lung epithelial cells exposed to pathogenic crystalline vs. amorphous silica.

Innate immune activation through sensing of asbestos and silica by the inflammasome in vivo was previously demonstrated. However, only myeloid cells were investigated with no further focus on the epithelium in vitro neither an attempt was made to localize the inflammasome in this in vivo model as well as in other animal models for silicosis. Large surface areas in many organs in contact with the environment, such as the epithelium, actively contribute to combat unwanted and potentially harmful influences leading to inflammation. Therefore, literature evidence that underscores a role for epithelial inflammasome signaling and immune homeostasis in mucosal barriers was reviewed in Chapter 5.

From the gene expression profiling in Chapter 3 we found indications of patterns associated with inflammasome signaling upon impingement of respirable silica in bronchial epithelial cells in vitro. This was further elaborated on in Chapter 6. Here became investigated if in these epithelial cells silica was sensed by the inflammasome and if, with respect to the disease phenotype of interstitial fibrosis, silica-induced functionality of this protein complex could have effect on proliferative capacity of fibroblasts.

In order to extrapolate our findings to an in vivo model exposure to different crystalline silica polymorphs on lung epithelial cells was tested in Chapter 7. Additionally, the objective was to demonstrate if impaired pulmonary function was associated with inflammasome-dependent 
inflammatory and fibrotic remodeling of lung tissue of rats exposed to silica in a surface reactivity dependent manner.

Chronic inflammation and fibrosis are typical hallmarks of silicosis. In order to translate research findings into clinical and patient outcomes, in Chapter 8, the aim was to localize various inflammasome-related proteins in healthy lung tissue, and further to determine the presence and functional activity of this danger sensor in the lung tissue of miners that developed silicosis compared to miners that did not.

Chapter 9 provides general discussion of thesis and outlines directions for future research. 



\section{CHAPTER 2}

\section{PATHOGENESIS AND MECHANISMS OF ASBESTOSIS AND SILICOSIS}

Paul M. Peeters ${ }^{1,2}$

Timothy N. Perkins ${ }^{1,2}$

Emiel F. M. Wouters ${ }^{1}$

Niki L. Reynaert ${ }^{1}$

Brooke T. Mossman ${ }^{2}$

${ }^{1}$ Department of Respiratory Medicine, Maastricht University Medical Centre, Maastricht University, Maastricht, The Netherlands

${ }^{2}$ Department of Pathology, University of Vermont College of Medicine, 89 Beaumont Avenue, Burlington, VT 05405, USA

In: Pathobiology of Human Disease. San Diego: Elsevier; 2014. p. 2654-2664. 



\section{GLOSSARY}

Asbestosis: a pulmonary fibrosis associated with exposure to asbestos and characterized by diffuse thickening of the interstitium of the lung.

Granulomas: small, well-organized nodules consisting of epithelioid macrophages and multinucleated giant cells found in chronic silicosis.

Inflammasome: a multi-protein cytoplasmic structure comprised of a NLRP or NALP3 domain, adapter protein complexes, and pro-caspase-1. Activation of the inflammasome causes activation of caspase-1 and release of mature interleukins-1 (IL-1), IL-18 and IL-33 from phagocytic cells, such as monocytes or macrophages, and bronchial epithelial cells.

Lung interstitium: the interior supporting structure of the lung comprised of matrix materials and surrounded by epithelial lining cells in individual respiratory units.

Phagolysosome: an intracellular vacuolar structure encompassing phagocytized fibers or particles after fusion with lysosomes.

Pneumoconiosis: a general term for fibrotic diseases of the lung occurring after exposure to a number of silicates or dust mixtures.

Silicosis: a group of interstitial lung diseases associated with exposure to crystalline silica and assuming several pathologies including alveolar proteinosis (filling of airspaces with lipoproteins), simple and chronic nodular disease.

\section{SUMMARY POINTS}

1. Asbestosis and silicosis are occupational diseases of the lung interstitium characterized by early epithelial injury and hyperplasia, accumulation of cells of the immune system, and the development of fibrogenesis. Once established, these diseases can progress in the absence of further exposures to asbestos fibers or silica particles. These particulates exist in different physicochemical forms that may be modified by interactions with other minerals or elements such as iron in the lung.

2. The pathological presentation of asbestosis vs. silicosis is complex and unique. The severity and extent of lesions may reflect initial deposition and durability of particulates, host susceptibility factors such as impaired clearance by smoking or infection, and immune responses governing lung repair. The intensity and duration of exposure as well as sites of accumulation of particulates also appear to play roles in governing susceptibility, development, and progression of lung disease.

3. Early epithelial cell injury, hyperplasia and/or repair after inhalation of these pathogenic particulates are becoming more apparent as critical features of response. Cross-talk between epithelial cells, alveolar macrophages and other cells of the immune system, and fibroblasts occurs via production of ROS/RNS, cytokines and chemokines. A critical mechanism sensing asbestos or silica as "danger signals" is the inflammasome which has been well-characterized in monocytes and macrophages, but less so in lung epithelial cells which also internalize particulates and have a functional inflammasome response.

4. Fibrogenesis is a complex process that involves components of the extracellular matrix that may be chemotactic towards inflammatory cells and fibroblasts. Circuitry between these cell types may lead to increases in fibroblast proliferation, collagen metabolism, disruption of epithelial and endothelial cell basement membranes, and aberrant ECM remodeling by cross- 
linking of collagen and reticulin fibers. An imbalance between MMPs and TIMPs promotes breakdown or increased production of matrix components.

5. The reasons for the different pathological presentation of asbestosis vs. silicosis may be related to initial responses of lung epithelial cells and can be dissected using modern techniques in molecular and protein biology. For example, different genes are over-expressed or under-expressed in response to crystalline silica or asbestos. Gene profiling studies also reveal common trends in expression of genes known to be critical to fibrogenesis. These changes, as well as release of cytokines and chemokines from epithelial cells, the cell types first encountering asbestos or silica after inhalation, are less striking after exposures to amorphous silica. 


\section{INTRODUCTION}

Exposure to naturally occurring airborne particulates from occupational and environmental sources is associated with a number of pulmonary abnormalities including fibrotic lung disease due to exposure to a number of distinct silicates. Originally, these diseases were referred to as pneumoconioses, a term collectively encompassing kaolinosis, talcosis, coal workers pneumoconiosis, etc., and categorized by workplace histories as well as accumulation of the respective minerals (kaolin, talc, coal mine dust, beryllium, hematite) in the lung. Of major concern both historically and presently is the inhalation of the known pathogenic minerals, crystalline silica and asbestos, distinct minerals with a number of polymorphs (Table 1) that are associated with the development of asbestosis and silicosis, irreversible and debilitating diseases developing in the lung interstium. Here, we focus first on the mineralogy and physicochemical characterstics of silica and asbestos types. Secondly, we describe the pathogenesis and distinctive features of these lesions including the cell types and products contributing to their development. Lastly, we emphasize common features and culprits of inflammation and fibrogenesis such as reactive oxygen and nitrogen species (ROS/RNS) and elaboration of cytokines and chemokines. Lastly, we present mechanistic data emphasizing unique gene and cytokine expression patterns that may explain the different histologic presentation of fibrotic lesions caused by silica and asbestos types.

Table 1: Types and polymorphs of asbestos and crystalline silica.

\section{Asbestos}

Serpentine

Chrysotile*: $\mathrm{Mg} 6 \mathrm{Si} 4 \mathrm{O} 10(\mathrm{OH}) 8$

Amphiboles

Crocidolite*: $(\mathrm{Na} 2(\mathrm{Fe} 3+) 2(\mathrm{Fe} 2+) 3 \mathrm{Si} 8 \mathrm{O} 22(\mathrm{OH}) 2$

Amosite*: (Fe,Mg)7Si8O22(OH)2

Tremolite: $\mathrm{Ca} 2 \mathrm{Mg} 5 \mathrm{Si} 8 \mathrm{O} 22(\mathrm{OH}) 2$

Anthophyllite*: (Mg,Fe)7Si8O22(OH)2

Actinolite: $(\mathrm{Ca} 2(\mathrm{Mg}, \mathrm{Fe}) 5 \mathrm{Si} 8 \mathrm{O} 22(\mathrm{OH}) 2$

Crystalline Silica Polymorphs: (all SiO2) with small percentages of other elements

Quartz*

Cristobalite*

Tridymite

Stishovite

Moganite

Coesite

Melanophlogite

*= Of present or past commercial value 


\section{MINERALOGY, CHEMISTRY, AND OTHER PROPERTIES OF ASBESTOS AND SILICA LINKED TO FIBROGENICITY}

Ironically, silica or silicon dioxide $\left(\mathrm{SiO}_{2}\right)$, is a mineral comprised of the two elements that account for the majority of the earth's crust. Sandblasting, hammer drilling, and tunneling were occupations of concern associated with inhalation of fibrogenic crystalline silica since the mid-1800s. Three forms of silica are considered to be of risk to human health (i.e. alpha quartz, cristobalite, and tridymite). Alpha quartz, hereafter referred to as quartz, is accountable for the majority of occupational exposures worldwide, as it is found in abundance in granite, sandstones, and most common types of rock. Little attention has focused on tridymite because of its rarity and lack of commercial value, whereas cristobalite, like tridymite, is formed from quartz at high temperatures in refractories and from diatomaceous earth. In contrast to these crystalline polymorphs, amorphous silica is less toxic and fibrogenic in experimental animals and man.

'Asbestos' is a commercial term for a number of both chemically and physically distinct silicate fibers (defined as having a greater than 3 to 1 length to diameter ratio (Table 1). Overall, they have been exploited worldwide in over 2000 industrial uses and occur in distinct localities. For example, chrysotile asbestos, the only member of the serpentine group of fibers, has been mined predominately in the Northern hemisphere, including the USA, Canada, and Russia. This type of asbestos accounts for over $95 \%$ of that used historically worldwide. In contrast, the amphibole group of asbestos encompasses 5 fiber types of which crocidolite and amosite have predominated in terms of their use and potency, especially in a unique asbestosassociated tumor, mesothelioma. They have been mined in Africa and Australia, among other countries, and were imported into the US and Britain during World War I and II. Amosite also exists in lungs of the US insulation workers, the largest occupational asbestos-associated cohort in the US, despite the fact that these workers were exposed predominately to chrysotile. These and studies with crystalline silica point to the common occurrence of mixed lung lesions and the importance of durability of crystalline silica and amphibole asbestos types. High acute and occupational exposure to crystalline silica dust or asbestos fibers is still a hazard worldwide in a number of third world countries where sandblasting, quarrying, abrasive blasting, construction, foundry work, ceramics, diatomaceous earth processing, and various types of mining are performed in the absence of permissible exposure levels. However, most countries have banned the commercialization, importation and use of asbestos, primarily due to its history in the induction of both lung cancers and mesothelioma.

\section{CLEARANCE AND DEPOSITION OF SILICA PARTICLES AND ASBESTOS FIBERS IN THE LUNG}

Upon the crushing, cutting and grinding of minerals and during their processing, respirable dust is created and inhaled. Silica particles, especially those with a diameter $>10 \mu \mathrm{m}$, are trapped in the upper airways and removed by mucociliary mechanisms. However, at high concentrations termed "overload", a fraction of the inhaled dust evades these clearance mechanisms. Particles or fibers $<5 \mu \mathrm{m}$ in diameter are thought to be most pathogenic, as they make their way to alveolar ducts and the alveoli. Their injury and interaction with a number of 
cell types, including epithelial cells that line the surface of the lung and alveolar macrophages (AMs) which accumulate at sites of particle or fiber deposition, initiates persistent inflammation and triggers fibrogenic responses described in detail below. In addition, long thin fibers, particularly amphibole types of asbestos, can align themselves with airways and be carried into the distal lungs. Some research suggests that fibers penetrate intercellularly through the epithelium or are transported into the interstitium of the lung by accumulating macrophages and neutrophils. Regardless, the accumulation and amounts of minerals over time are linked to the development of silicotic nodules and diffuse interstitial fibrosis associated with high asbestos exposures. Research on patients exposed to a number of silicates suggests that particles and fibers are translocated to the periphery of the lung over time. It is also widely acknowledged that chrysotile fibers can break down in the lung into smaller fibrils, a process facilitated by the acidic $\mathrm{pH}$ of phagolysosomes, intracellular structures encompassing fibers after cell uptake.

\section{PATHOLOGICAL PRESENTATION AND DIAGNOSIS OF SILICOSIS AND ASBESTOS \\ Silicosis}

Acute silicosis, or silico-proteinosis, rapidly develops in just a few months to a few years after initial exposure to silica dusts, and is usually seen after high exposures to freshly fractured dusts as in sandblasting and abrasives. Patients usually present with extreme shortness of breath and rapid decreases in respiratory function. Acute silicosis presents with excessive protein-rich, eosinophilic exudates in the alveolar space, interstitial inflammation, and the development of pulmonary lesions. Similar to the acute form of silicosis, accelerated silicosis presents with pulmonary edema and inflammation, as well as granuloma formation, alveolar epithelial cell hyperplasia, and collagen-rich silicotic nodules. Acute and accelerated silicosis have a rapid onset. In contrast, chronic silicosis (simple), a latent, more severe form of pulmonary fibrosis, can develop anywhere from 10 years to multiple decades after initial exposure. The hallmark of disease is the presence of characteristic silicotic nodules, which are distinctive lesions of acellular, concentric collagen fibers, surrounded by fibroblasts, immune cells, and connective tissue (Figure 1). Often, use of polarized light microscopy on tissue sections reveals weakly bi-fringent silica particles within nodules and surrounding dust-laden cells. Lesions in simple silicosis, are $\leq 1 \mathrm{~cm}$ in diameter and can be seen as distinct opacities on chest CT scans. In addition, patients tend to have signs of airflow obstruction via spirometry analyses. Simple silicosis can progress to conglomerate silicosis in which the characteristic nodules grow and coalesce with others to form larger silicotic nodules that continue to progress to a growth of $\geq 2 \mathrm{~cm}$ in diameter. This results in progressive massive fibrosis (PMF), an extremely severe form of disease with destruction of the normal respiratory architecture and rapid diminshment of proper lung function of which there is no known cure. In silicotuberculosis, silicotics tend to have increased susceptibility to respiratory infections (e.g. M. tuberculosis), as well as other mycobacteria, and respiratory pathogens. 


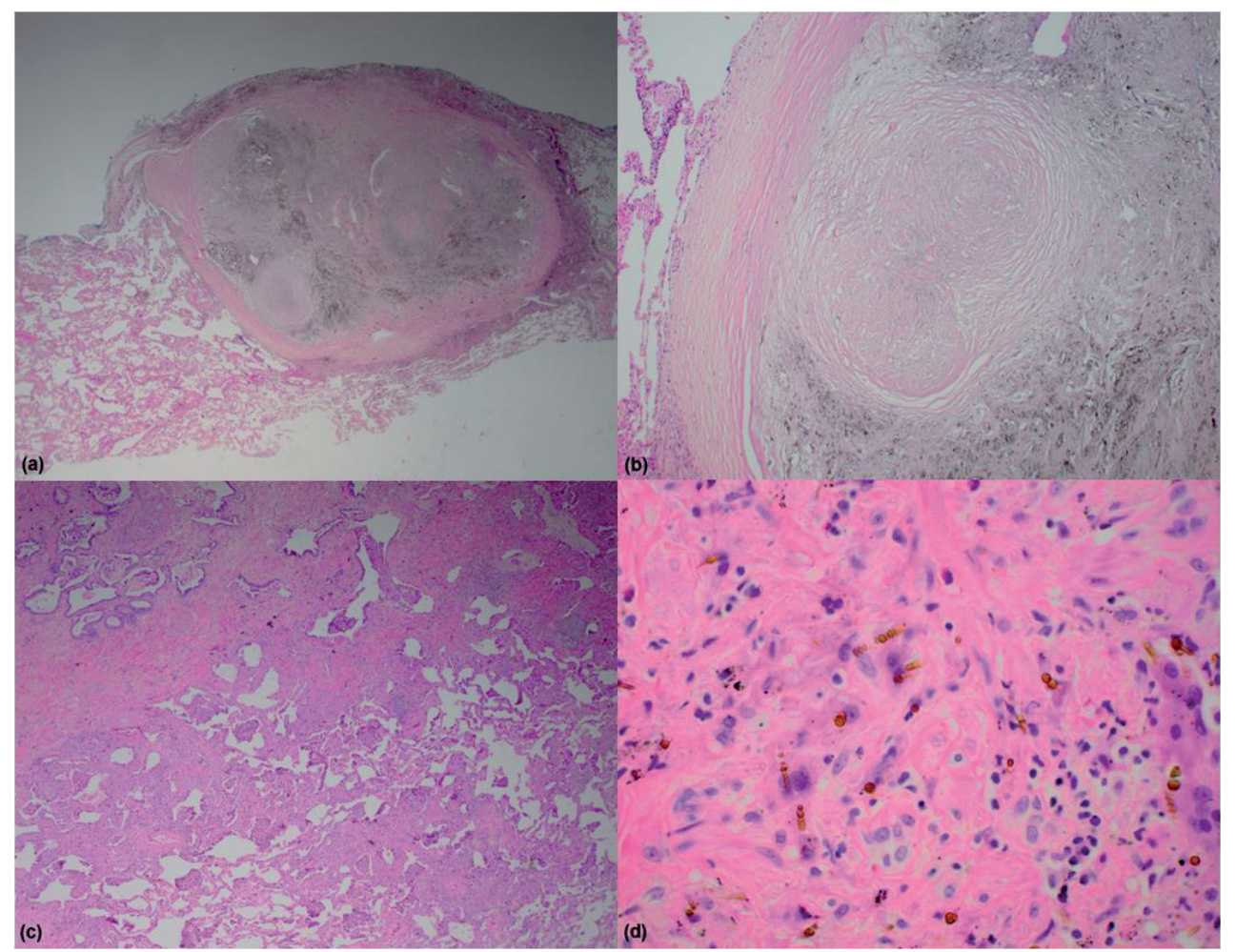

Figure 1: Histological presentation of silicosis and asbestosis. (a) A circumscribed white-gray, acellular silicotic nodule. The nodule, which contains silica and some carbonaceous material, is surrounded by a cellular capsule comprised primarily of AMs and other cell of the immune system. X 200; (b) Progressive massive fibrosis illustrated by coalescence of two acellular nodules. Note thick layer of ECM to left of nodules as well as cellularity and particle deposition to the right. X 1000; (c) Advanced asbestosis in which increased ECM occurs diffusely throughout the lung. Note the occasional bronchioles and airspaces filled with inflammatory exudates. X 200; (d) Complete obliteration of an asbestotic lung by ECM. This illustration also shows marked accumulation of interstitial inflammatory cell types and asbestos (ferruginous) bodies that are copper-colored and represent fibers coated with iron. $X$ 600. All illustrations were kindly provided by Kelly Butnor, MD, a board-certified pathologist at the University of Vermont.

\section{Asbestosis}

The severity of asbestosis depends on the type and duration of exposure as shown in various cohorts of asbestos workers, the concentration of dust in the work place, and the fiber "burden" or amount in the lung. Often disease is clearly evident on gross examination of lungs, and is chronic and progressive. In contrast to silicosis that occurs more proximally in the lung, asbestosis arises most often in the lower lobes, observations that may reflect differences in initial deposition or accumulation of each particulate. Early lesions are thought to reflect thickened walls of respiratory bronchioles with only occasional involvement of pulmonary units and discrete foci with accumulations of asbestos bodies, i.e. iron-coated or ferruginous bodies thought to represent accumulation of hemosiderin from macrophages on 
long, primarily amphibole fibers. Whether this is a protective or destructive mechanism integral to lung injury is unclear. However, diagnosis of asbestosis requires demonstration of both asbestos bodies and histologic demonstration of fibrosis. As lung disease progresses, the pulmonary subunits are affected in a radial fashion, and scarring progresses, obliterating airspaces with fibrotic tissue. Often asbestosis is detected by radiology or computer tomography (CT), less invasive techniques, and grading systems for the severity and extent of abnormalities have been developed by pathologists and radiologists.

\section{Animal Models of Asbestosis and Silicosis}

Insights into the pathogenicity and elucidation of cellular and molecular responses to silica and asbestos largely stem from experimental animal models. These studies indicate that both silica and asbestos induce a rapid inflammatory response. The concentrations, lengths and methods of exposure as well as surface area characteristics of particles and fibers are critical determinants in the development and progression of disease. Rats appear to be the most commonly used animal species for studying asbestos- and silica-induced effects because of their propensity to develop fibrogenicity and lung cancers. However, their short life span does not reflect important differences in fiber or particle durability that are thought to be important in the long latency periods of human disease. Both silicosis and asbestosis can be induced by intratracheal instillation, nasal or oropharangeal aspiration or by inhalation of mineral particles. Since the former methods of exposure bypass normal clearance mechanisms occurring after inhalation, different patterns and mechanisms of inflammation and disease have been observed in these models. For example, whereas apoptosis, i.e. programmed cell death, predominates in instillation models, a number of anti-apoptotic markers occur in lung after inhalation of silica, suggesting mechanisms of lung defense. Increased expression of antioxidant enzymes, particularly the mitochondrial superoxide dismutase, SOD2, are also observed in type II epithelial cells after inhalation of either asbestos or silica. These presumed defense mechanisms occur before the advent of fibrogenic lesions and persist for many months after cessation of exposures. Increased expression and elaboration of proinflammatory cytokines occur earlier in an intratracheal instillation model of acute silica exposure, but were biphasic and less pronounced in an inhalation model of chronic silicosis.

Silica-induced lung fibrosis in rats is strongly related to chronic inflammation and can be ameliorated by treatment with anti-inflammatory agents. This process encompasses the activation of alveolar macrophages and enhanced humoral and cell-mediated immunities. Rats exposed for 3 to 6 months typically show aggregates of macrophages appearing at interstitial, subpleural, and peribronchiolar locations. These changes occur parallel with septal remodeling, granulomatous lesions and focal fibrosis. Furthermore, enlarged lymph nodes with associated increases in lymphocyte numbers have been described. Intratracheal administration of silica results in increased levels of cell adhesion molecules in lung tissue as well as in BALF fluid of mice, followed by a marked neutrophil influx to the lung. Mice exposed to silica have a massive inflammatory response with proinflammatory cytokines and chemokines as key contributors of the pathogenesis. Silica-induced nuclear factor kappa B $(\mathrm{NF}-\kappa \mathrm{B})$ dependent gene expression in vivo through the use of luciferase reporter mice 
exposed to quartz. Systemic inhibition of NF- $\kappa$ B activation using a pharmacologic inhibitor of I $\kappa$ B alpha phosphorylation decreases silica-induced inflammation, immune cell recruitment and collagen deposition. Recently, recruitment of IL-17A-producing $\mathrm{T}$ cells in the lung mediated by macrophage-derived IL-23, has been associated with experimental silicosis induced by intratracheal instillations in mice. This study indicates that acute alveolitis induced by silica is IL-17A dependent. However, several studies report that exacerbated lung inflammation is not always followed by increased lung fibrosis, emphasizing the importance of lung defense mechanisms.

\section{Cellular and molecular mechanisms of asbestosis and silicosis}

\section{Reactive oxygen and nitrogen species (ROS/RNS)}

Quartz exposure by intratracheal injection elicits increased ROS production in lung tissue compared to rats receiving nonpathogenic titanium dioxide. Introduction of free radical scavengers, including antioxidant enzymes, in this model and in inhalation studies using asbestos prevent lung injury, inflammation and fibrogenicity. Thus, the enhanced formation of ROS can be considered as a primary event in disease development. Oxidants also are produced in abundance by freshly fractured silica and generated by iron-driven reactions on asbestos fibers. For example, crystalline silica particles produce particle-derived ROS by interaction of particles with molecular oxygen and other surrounding molecules. During crushing and grinding of silica-containing materials, the reactive sianols $(\mathrm{SiOH})$, and dangling bonds $\left(\mathrm{SiO}^{\circ}\right)$ generated at the surface in cleavage planes may interact with molecular oxygen to produce surface radicals $\left(\mathrm{SiO}_{3}{ }^{-}\right.$or $\left.\mathrm{Si}^{+}{ }^{-} \mathrm{O}_{2}{ }^{\circ}\right)$, as well as particle-derived $\mathrm{ROS}$ such as hydrogen peroxide $\left(\mathrm{H}_{2} \mathrm{O}_{2}\right)$ and damaging hydroxyl radicals $\left(\mathrm{HO}^{\circ}\right)$. It has been established that freshly fractured silica dust has an increased pathogenic potential compared to aged silica dust because surface reactivity diminishes over time as reactive surface molecules dissipate.

Excessive oxidant production in the lungs upon exposure to silica or asbestos is also associated with increases in lipid peroxidation and inflammation. Lipid peroxidation is observed in lung tissues in experimental animal models and in BALF cells in humans exposed to silica or asbestos. These data suggest that oxidants are intrinsic to the damaging effects of these pathogenic particles after inhalation.

An intrinsic antioxidant defense system, comprised of various antioxidant enzymes and oxidant scavengers, is intended to keep the oxidative environment balanced. As noted above, a number of antioxidant enzymes are induced or structurally altered by inhalation of crystalline silica or asbestos including superoxide dismutases (SODs), heme oxygenase-1 (HO-1), peroxiredoxins (PRXs), glutathione (GSH), and glutathione peroxidase (GPX). Thus, at high concentrations of particulates, the overall balance between oxidative stress, i.e. the production of fiber/particle-and cell-derived oxidants as well as dysregulation of antioxidant defenses, prevails. 


\section{Importance of inflammatory cell types}

In animal inhalation models of exposure, the alveolar macrophage (AM) is one of the first cell types to accumulate at points of particle or fiber deposition. For these reasons and because they elaborate a plethora of chemokines and cytokines affecting a number of cell types, AMs are regarded as a pivotal cell type in development of pulmonary inflammation that is critical to fibrogenesis. AMs engulf silica particles via scavenger receptors such as SR-AI (scavenger receptor AI) or macrophage receptor with collagenous structure (MARCO/SRAII), as well as FcyIIR. Phagocytosis can stimulate NADPH oxidases, especially during frustrated phagocytosis of long asbestos fibers, leading to production of ROS/RNS. In addition, damage or activation of macrophages and surrounding cells may occur via particle or fiber-derived oxidants.

Macrophages are a "two- edged sword" as they clear fibers or particles from the lung via mucociliary mechanisms, or transport to them to lymphatics. Alternatively, they can release damaging proteases and other enzymes, chemokines, cytokines, and other factors upon cell death and lysis. These events are thought to perpetuate influx of other immune populations, particularly neutrophils and a prolonged inflammatory response. For example, increased expression of Tumor Necrosis Factor- $\alpha(\mathrm{TNF} \alpha)$ and IL-1, promotes the production of acute phase response proteins, leukocyte chemoattractants, and fibrogenic mediators. Epithelial cell proliferation, recruitment of fibroblasts, and excessive collagen deposition, has also been attributed to factors released from AMs.

Potent cytokine-induced neutrophil chemoattractants (CINC) also have been implicated in experimental silicosis. Of the four isoforms reported, three contribute to neutrophil recruitment in the alveolar space and granuloma formation (CINC-2 $\alpha$, CINC-2 $\beta$, and CINC3). Macrophage inflammatory proteins (MIPs) are a family of chemokines,secreted by macrophages and epithelial cells in response to silica or asbestos exposure. MIP-1 $\alpha$ and $\beta$ are chemoattractants for monocytes and lymphocytes, while MIP-2 is a potent neutrophil chemoattractant. Interleukin-10 (IL-10) has been shown to have an anti-inflammatory effect in experimental silicosis, as there is less inflammatory cell infiltration and cytokine or chemokine release in the lungs. However, there may also be a profibrotic effect as IL-10 increases the expression of TGF $\beta$, a critical cytokine in models of asbestosis and silicosis. This potent signaling molecule has a multiplicity of effects that promote fibroblast responses and deposition of collagen.

Recent evidence indicates that the binding of crystalline silica to scavenger receptors leads to cell death of AMs and release of proinflammatory chemokines and cytokines contributing to lung inflammation and fibrosis. This form of inflammatory cell death is called pyroptosis and is accompanied by secretion of the pro-inflammatory cytokines IL-1 $\beta$, IL-18, and alarmins such as IL-33, HMGB1 and S100-proteins in a process linked to activation of the inflammasome that has been reported in monocytes and THP-1 macrophage-like cells in response to asbestos or silica. The NLRP3 inflammasome is causally related to cell inflammation and lung injury as verified in asbestos inhalation experiments using wild-type 
vs. NLRP3 inflammasome knock-out mice. Pyroptosis, or cell swelling, particularly of ciliated epithelial cells, has been documented previously by asbestos in tracheal explants. Studies suggest that proteins and proteases and free particles or fibers are released by this process can then be re-engulfed by AMs and other cell types in a vicious cycle of prolonged inflammation.

Mast cells also have a vital role in the development of pulmonary inflammation in silicosis and asbestosis. Mast cells are crucial to the induction of pulmonary neutrophilia, ROS production, protease release (e.g. chymase), as well as pro-inflammatory cytokine and chemokine secretion. Mast cells secrete significant amounts of TNF $\alpha$, IL-13, and monocyte chemo-attractant protein-1 (MCP-1), promoting neutrophil, lymphocyte, and monocyte/macrophage recruitment in response to silica. Mast cells, like macrophages, interact with silica particles via scavenger receptors, e.g. (SR-AI), which in part, mediate secretion of TNF $\alpha$ and ROS generation, as well as apoptosis, which further potentiates inflammation and tissue damage.

The role of lymphocytes in silicosis and asbestosis is still not well understood. However, lymphocytes, albeit smaller in number in BALF than macrophages and neutrophils in experimental animal models, are clearly attracted to lung and stimulated by both particulates. They also can be seen encompassing silicotic nodules. The lymphocyte response seen in silicosis is mostly $\mathrm{CD}^{+}, \mathrm{CD}^{+}$, and $\mathrm{NK}$ cells and is driven towards a $\mathrm{T}_{\mathrm{H}} 1$ response with increases in IL-12, IL-18, and IFN $\gamma$. The production of IFN $\gamma$ likely enhances the inflammatory response by increasing $\mathrm{TNF} \alpha$ through $\mathrm{NF} \kappa \mathrm{B}$ activation in macrophages; however, it also has an inhibitory effect on TGF $\beta$ and fibroblast responses.

\section{The lung epithelium, a key player in fibrogenesis}

The epithelial cells of the lung have a vital role in airway defense, as they are the first target cells to come in contact with inhaled foreign materials. If particles cannot be cleared by normal mucociliary mechanisms, epithelial cells can be injured or stimulated. The epithelium is heavily involved in cell to cell signaling, in which they release -cytokines and growth factors. Additionally they respond to factors released by surrounding macrophages and other cell types that have been activated, injured, or recruited to sites of particle deposition and injury.

Recently, much focus has been placed upon the role of lung epithelial cells in fibrogenesis. Like AMs, they phagocytose silica and asbestos, and recent work from our laboratories suggests that they also have functional inflammasomes. Type II cell hyperplasia in animal models is an early event following type I epithelial cell damage by both minerals, and hyperplasia of both tracheobronchiolar and alveolar type II epithelial cells is a precursor to metaplasia and tumor formation in organ explants and rat models of particle or fiber-induced lung cancers. Normally, epithelial cell proliferation is induced after injury to restore a functional epithelial cell barrier to toxins. However, unregulated epithelial proliferation can lead to hyperplasia, and plays a critical role in fibrotic and mitogenic responses. After 
exposure to silica or asbestos, factors produced by AMs and direct interaction of epithelial cells with long asbestos fibers stimulate induction of S-phase cells and DNA synthesis in both bronchiolar and alveolar type II epithelial cells. Cell signaling cascades strongly associated with increases in S-phase cells and epithelial cell proliferation include mitogen activated protein kinases (MAPK) pathways and subsequent activation of early response protooncogenes comprising the AP-1 transcription factor complex. In effort to restore a functional epithelial barrier, it is possible that a lack of control of these cell-signaling pathways occurs in response to asbestos or silica.

Growth factors released by macrophages such as platelet-derived growth factor (PDGF), insulin growth factors (IGF), and fibroblast growth factors (FGF) are associated with the induction of epithelial cell proliferation by both minerals. Epithelial cells are also capable of secreting such factors which may contribute to an autocrine and/or paracrine response. For example, alveolar type II epithelial cells synthesize macrophage inflammatory protein (MIP2 ), both in vitro and in vivo in response to asbestos or silica. Alveolar epithelial cells in vitro also release mitogens including PDGF-like, IGF-1-like, and FGF-like molecules. These agents are involved in epithelial repair as well as alveolar type II cell hyperplasia and fibrogenesis. In addition to production of a number of factors increasing inflammation and autocrine cell proliferation, lung epithelial cells secrete basic fibroblast growth factor (bFGF) that promotes fibroblast proliferation in a transforming growth factor- $\beta$ (TGF- $\beta$ ) model of epithelial-mesenchymal transition. Use of anti-bFGF antibodies significantly attenuates experimental silicosis, and bFGF also is associated with increased numbers of mast cells in silicotic lungs.

Affymetrix gene profiling analysis and multiplex immunoassays for chemokines and cytokines in cell medium have been used by our laboratories to identify increased mRNA levels and release of chemokines and cytokines from human bronchial epithelial cells in vitro. These data indicate epithelial responses to cristobalite silica that include: 1) key inflammatory chemokines and cytokines, including bFGF; 2) matrix metalloproteinases and other genes related to dissolution of the extracellular matrix and tissue remodeling; 3) genes that participate in wound healing, stress responses, and immune responses; 4) genes related to cell signaling; and 5) genes linked to antioxidant defense such as SOD2. Dose-related expression and secretion of similar chemokines and cytokines were not observed with equal surface area doses of amorphous silica. Moreover, both types of silica had comparable effects on the viability of human bronchial epithelial cells, challenging the paradigm that toxicity per se can predict pathogenicity of particulates. In recent work, we have also established that both commonalities and differences exist in gene profiling of normal human bronchial epithelial cells in vitro to crocidolite asbestos or cristobalite silica. Common changes included upregulation of genes linked to signaling cascades including the mitogen-activated protein kinases (MAPK), activator protein-1 (AP-1), and NF- $\kappa \mathrm{B}$, stress/inflammatory-response signaling, ECM dissolution, and cell migration, as well as genes governing a number of cellular defense mechanisms. In opposition to silica, asbestos caused increased expression of genes linked to cell injury, small molecule biochemistry (including genes encoding enzymes 
involved in detoxification of xenobiotics), alterations in tissue morphology, and cell proliferation, tumorigenesis, and metastasis. Uniquely upregulation of genes at higher concentrations of silica were those encoding membrane receptors (FAS, TNFRs) previously reported in models of silicosis, receptors linked to inflammation and connective tissue remodeling, and mediators of cell injury and proliferation induced by oxidative stress. Decreased mRNA levels of genes linked to cell survival and apoptosis as well as increases in expression of genes governing interferon signaling were observed uniquely with silica.

Silica can initiate apoptosis in both AMs and lung epithelial cells in intratracheal instillation models, but apoptosis has not been noted in inhalation models using asbestos or silica. In vitro, asbestos and silica-induced apoptosis is typically mediated through a complex chain of cellular responses leading to caspase activation. Activated caspases are governed by a coordinated hierarchy of initiator caspases (e.g., caspases 8 and 9) that activate an effector caspase (e.g., caspase 3). These are responsible for apoptosis via proteolytic cleavage of homeostatic and structural proteins. The physiologic consequences of silica- or asbestosinduced apoptosis in epithelial cells of the lung in vivo are still speculative, but a recent study suggests that apoptosis is a major pathway responsible for the removal of proliferating alveolar type II epithelial cells in acute lung injury.

\section{Fibroblasts as effector and target cells in fibrosis}

Lung fibroblasts produce a number of inflammatory and other mediators in fibrogenesis and are a major source of collagen and other interstitial matrix components. As emphasized above, a hallmark of the development of fibrosis is the replacement of normal pulmonary parenchyma with a collagen matrix. However, particles deposited in the alveoli may also reach fibroblasts directly at places where the permeability of the epithelial barrier is increased or at sites where the epithelium has been damaged and or replaced by migrating fibroblasts. In asbestosis and silicosis, significantly increased type I collagen exists in fibrotic tissues as compared to normal tissues. The net amount of collagen deposited by fibroblasts is regulated continuously by collagen synthesis and collagen catabolism. The turnover of collagen and other extracellular matrix (ECM) proteins is also controlled by various matrix metalloproteinases (MMPs) and their inhibitors (tissue inhibitors of metalloproteinases (TIMPs).

Both silica and asbestos stimulate lung fibroblasts both directly, by increasing cell proliferation, and indirectly, by stimulating the release of factors (TNF- $\alpha$, TGF- $\beta$, etc.) from activated AMs. These factors increase proliferative and biosynthetic activities of fibroblasts and also lead to an increase in collagen and IL-6 production. When exposed to pathogenic mineral dusts, fibroblasts produce a diverse array of inflammatory mediators, including interleukin-8 (IL-8), MCP-1, IL-6, cyclooxygenase-2 (COX-2), prostaglandins (PGE synthase and PGE2) and transforming growth factors (TGF- $\alpha$ and TGF- $\beta$ ). IL-1 and PDGF as well as fibronectin and type 1 insulin-like growth factor (IGF-1) have been reported to increase fibroblast proliferation and fibrogenesis by both asbestos and silica. Secretion of a number of growth factors that promote the migration, activation, differentiation, and proliferation of 
fibroblasts also has been demonstrated in both asbestos and silica- exposed cells and lung tissues. It is generally acknowledged that secreted cytokines from a number of cell types promote pulmonary fibroblasts to proliferate and synthesize excess collagen, which directly contributes to formation of silicotic nodules and interstitial fibrosis.

Fibrogenic responses to asbestos or silica may be similar in the initiation of fibrogenesis, but different in the establishment of lung lesions which are dissimilar and distinctive (Figure 1). Abnormal wound healing in response to ongoing alveolar epithelial injury at the site of impingement of inhaled particulates and activation of repair mechanisms is associated with the formation of patchy fibroblast-myofibroblast foci. Driving fibroblast responses instigates the deposition of collagen and various ECM components, leading to the development of collagen-rich nodules in silicosis or diffuse interstitial fibrosis observed in asbestos. The main growth factors involved in these processes include, TGF- $\beta$, PDGF, IGF-1, bFGF, and epidermal growth factors such as EGF or TGF- $\alpha$. AMs and monocytes exposed to silica or asbestos release key pro-inflammatory cytokines such as TNF- $\alpha$ and IL- $1 \beta$ which directly stimulate fibroblast activation and pulmonary deposition of matrix proteins. For example, IL1 is described as a direct stimulant of collagen production and fibroblast response.

As described above, some studies support the notion that apoptosis contributes to the pathogenesis of lung fibrosis in robust models such as injection of bleomycin or upregulation of Fas on the lung epithelium that is thought to induce epithelial apoptosis as a prelude to fibrogenesis. This process then leads to accumulation of mesenchymal cells including abnormal fibroblast populations emerging in the interstitium and further migrating into the alveolar spaces. These emerging fibroblast subpopulations may be further catalysts for tissue damage, wound healing and fibrogenesis as they are the main cell types producing collagen fibers, elastin proteins, and mucopolysaccharides.

\section{Dissolution and deposition of extracellular matrix (ECM)}

Under normal conditions, the turnover of ECM is tightly controlled to maintain morphostasis. ECM includes proteins such as laminins, entactin, collagens (I, III, V, VI, and VII), fibronectin, elastin, hyaluronan and proteoglycans that serve as a supporting matrix for the lung architecture, but also have chemotactic activity towards inflammatory cells and fibroblasts. Interstitial fibrosis is characterized by fibroblast activation and aberrant ECM remodeling with significant discrepancies in quantity and composition as well as accumulation of these proteins in the interstitial and alveolar spaces. These changes occur with disruption of epithelial cell and endothelial cell basal laminas. Many studies have demonstrated that elastases, gelatinases and cathepsins participate in the breakdown of ECM during asbestosis or silicosis. Therefore, these diseases can be conceptualized as consequences of failure in regulation of synthesis and degradation of ECM molecules.

In experimental models of silicosis and asbestosis, fibroblast-stimulating factors are released that promote hyalinization and collagen deposition, thus leading to an increase in deposition of excess lung collagen and increased hydroxylproline. Changes in cross-linking of lung collagen and reticulin fibers seem to be common alterations in all animal models of interstitial fibrosis. Both asbestosis and silicosis are accompanied by increased deposition and 
degradation of connective tissue matrix material as governed by MMPs and their inhibitors (TIMPs). A number of fibrogenic cytokines regulate these enzymes as well as NFkB and AP1 transcription factors that tightly regulate their enzymatic activity at the transcriptional level. Modulation of MMP secretion also occurs by cytokines such as IL- 1 , TNF- $\alpha$ and TGF- $\beta$. It is assumed that TIMPs are involved in a compensatory mechanism to counter increased MMP levels by binding at the active sites of these enzymes and thereby rendering them enzymatically inactive. Imbalances in the expression of matrix MMPs and TIMPs are likely integral to ECM remodeling and basement membrane disruption during fibrogenesis. Additionally, MMPs as ECM degradation enzymes, can lead to a favorable environment for tumorigenesis and proliferation of tumor cells. In this regard, an ongoing debate is the role of asbestos-induced fibrosis in carcinogenesis as some investigators regard fibrosis as a necessary process in creating a favorable environment in the lung for the development of tumors.

\section{Cell signaling in lung inflammation and fibrogenesis}

Both asbestos and crystalline silica exposures cause activation of a number of cell signaling pathways, which in turn induce the activation of key transcription factors. The key signaling pathways include protein kinase $\mathrm{C}$ (PKC), the mitogen-activated protein kinases (MAPKs), and the phosphoinositide-3 kinase (PI3K) pathway. The activation of these signaling cascades leads to the activation of the well known transcription factor complexes, AP-1 and NFkB.

Silica exposures in vitro induce activation of extracellular signal regulated kinases (ERKs1/2), c-jun N-terminal kinases ( $\mathrm{JNKs}$ ), and p38 kinases in an oxidant-dependent manner via upstream activation of protein kinase c (PKC). In contrast, asbestos fibers appear to preferentially stimulate ERKs1/2 and 5 in lung epithelial cells that most often occur after phosphorylation of the EGF receptor. However, oxidant-dependent activation of ERKs can be facilitated by Src protein kinases, PKC and protein kinase D (PKD). A key event is nuclear translocation of ERKs and transcription of early response genes (e.g. jun, fos, fra-1) whose proteins comprise the AP-1 transcription factor. AP-1 activation is linked to increased expression of a number of proinflammatory cytokines (e.g. IL-8), proteases (e.g. MMPs), transcription factors/regulators (e.g. jun, fos, egr1/2), and transcription of cell cycle progression molecules such as. cyclin $\mathrm{D}_{1}$. Thus, AP-1 transactivation is a key, common pathway that mediates inflammation, extracellular matrix (ECM) dissolution, and cell proliferation.

Activation of the NFKB transcription factor complex is another crucial event in both asbestos and silica-induced lung disease. Through activation of the PI3K signaling pathway, as well as a number of protein tyrosine kinases (PTKs), pathogenic minerals cause nuclear translocation of active subunits of $\mathrm{NF \kappa B}$, a process known to consequently up-regulate levels of potent inflammatory cytokines (e.g. TNF $\alpha$, IL-1, MCP-1). Use of various antioxidants impairs activation of $\mathrm{NF \kappa B}$, release of inflammatory molecules, and neutrophil recruitment. This pathway is induced by both asbestos fibers and crystalline silica particles in key cell types in fibrogenesis including AMs, bronchial and alveolar epithelial cells, and fibroblasts. A schematic diagram indicating the role of these pathways in promulgating the production of chemokines, cytokines and other events in fibrogenesis is provided in Figure 2. 


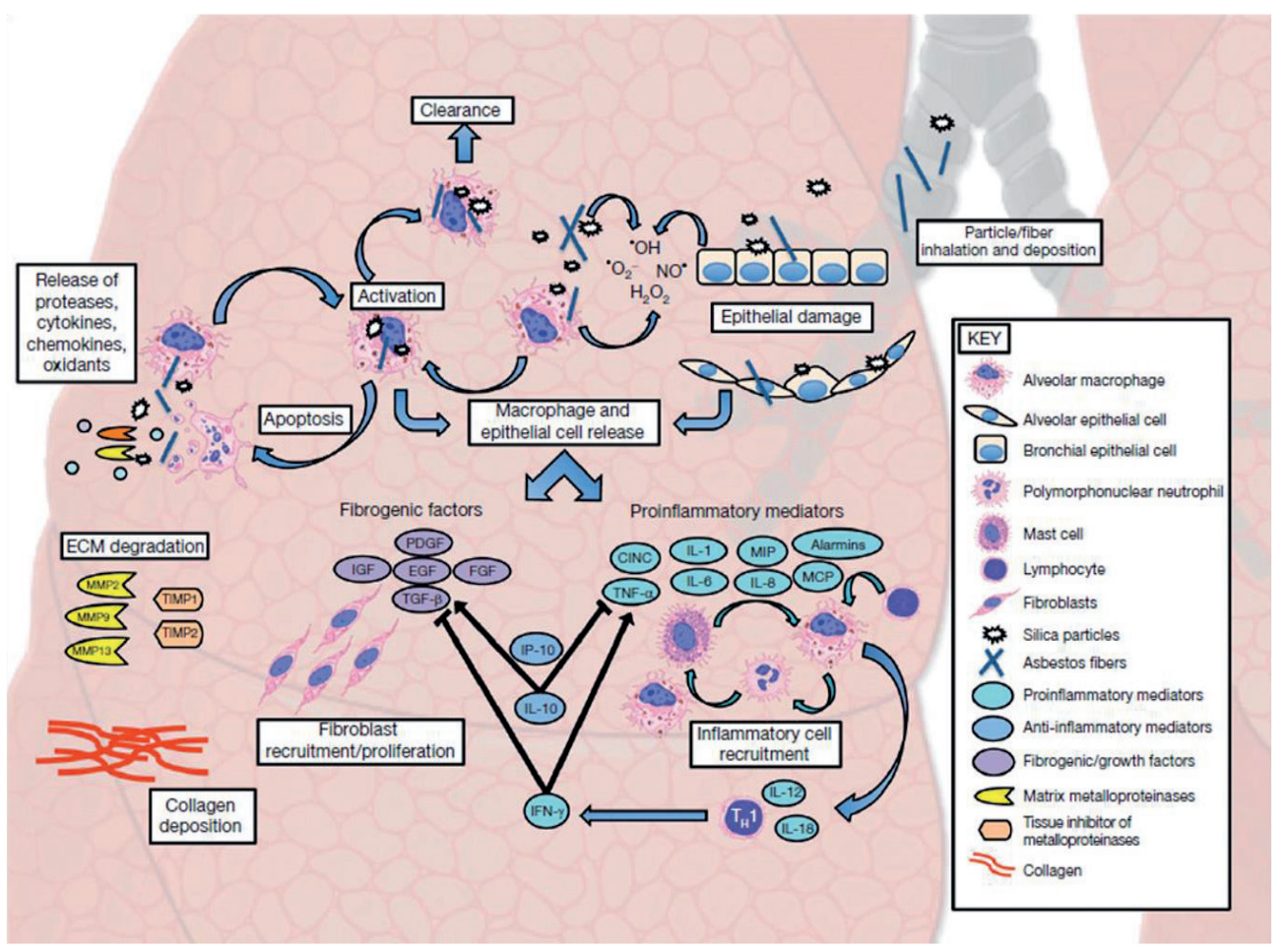

Figure 2. Schematic diagram of commonalities in asbestos- and silica-induced pulmonary fibrosis. Upon inhalation of crystalline silica particles or asbestos fibers $(<10 \mu \mathrm{m}$ in diameter), these minerals infiltrate the distal airways and alveoli. Particles initially interact with the bronchial and alveolar epithelium and resident macrophages. Macrophages are activated upon interaction with and engulfment of particles, releasing many common proinflammatory mediators (e.g. IL-1, TNFa), fibrogenic factors (e.g. TGF $\beta, F G F$ ), and ROS/RNS. This promotes inflammatory cell recruitment and fibroblast proliferation. AMs which attempt to phagocytose particulates clear the particles from the lung at low exposures or undergo cell death at high exposures. Upon death of AMs or epithelial cells by lysis, apoptosis or pyroptosis, damaging proteases, cytokines, chemokines, and oxidants are released, and free particles and fibers recirculated to other cell types, promoting cycles of prolonged inflammation. In addition, damage to the epithelium also induces the release of a number of proinflammatory and fibrogenic factors, promoting chemotaxis of inflammatory cells and fibroblasts, as well as epithelial cell proliferation and hyperplasia.

Abbreviations : nitric oxide $\left(\mathrm{NO}^{*}\right)$ hydroxl radical $(\mathrm{OH})$, superoxide $\left(\mathrm{O}_{2}{ }^{-}\right)$, hydrogen peroxide $\left(\mathrm{H}_{2} \mathrm{O}_{2}\right)$, cytokine induced neutrophil chemoattractant (CINC), Macrophage inflammatory protein (MIP), macrophage chemoattractant protein-1 (MCP-1), tumor necrosis factor-alpha (TNFa), interleukin-1 (IL-1, IL-6, IL-8, IL-10, IL-12, IL-18), interferon-gamma (IFN $)$ ), Interferon-gamma induced protein-10 (IP-10), platelet-derived growth factor (PDGF), epidermal growth factor (EGF), insulin-like growth factor (IGF), fibroblast growth factor (FGF), transforming growth factor-beta (TGF $\beta$ ), matrix metalloproteinases (MMPS), Tissue inhibitor of metalloproteinases (TIMPS). 


\section{SUMMARY}

Asbestosis and silicosis are the two most studied lesions of a number of pneumoconioses associated with accidental or workplace inhalation of silicates. Asbestos and silicas are fibers and particles, respectively, comprised of a number of types or polymorphs. Data from animal and cell culture models as well as clinical studies suggest that a number of cell types, namely lung epithelial cells, cells of the immune system (AMs, neutrophils, mast cells) and fibroblasts are affected directly by fiber or particle contact and uptake. Injury also may occur indirectly via ROS production occurring on fiber or particle surfaces. Common and repeated cycles of fiber/particle uptake, inflammation and deposition within the lung interstitium may perpetuate the development of asbestosis and silicosis. However, the pathologic presentation of both diseases is distinct and may progress in the absence of further exposures to minerals. There are no effective regimens for treatment or cure of these diseases although glucocorticoids and anti-inflammatory agents are used currently, and new agents may be indicated based upon novel observations such as inflammasome activation and gene profiling that may be linked to early disease. 


\section{FURTHER READING}

\section{Reviews}

American Thoracic Society, 2004. Diagnosis and initial management of nonmalignant diseases related to asbestos. American journal of respiratory and critical care medicine. 170, 691-715.

Bernstein, D. M., Hoskins, J. A., 2006. The health effects of chrysotile: current perspective based upon recent data. Regulatory toxicology and pharmacology : RTP. 45, 252-64.

Castranova, V., 2004. Signaling pathways controlling the production of inflammatory mediators in response to crystalline silica exposure: role of reactive oxygen/nitrogen species. Free radical biology \& medicine. 37, 916-25.

Castranova, V., Vallyathan, V., 2000. Silicosis and coal workers' pneumoconiosis. Environmental health perspectives. 108 Suppl 4, 675-84.

Craighead, J. E., et al., 1982. The pathology of asbestos-associated diseases of the lungs and pleural cavities: diagnostic criteria and proposed grading schema. Report of the Pneumoconiosis Committee of the College of American Pathologists and the National Institute for Occupational Safety and Health. Archives of pathology \& laboratory medicine. 106, 544-96.

Craighead, J. E., Mossman, B. T., 1982. The pathogenesis of asbestos-associated diseases. The New England journal of medicine. 306, 1446-55.

Ding, M., et al., 2002. Diseases caused by silica: mechanisms of injury and disease development. International immunopharmacology. 2, 173-82.

Fubini, B., Hubbard, A., 2003. Reactive oxygen species (ROS) and reactive nitrogen species (RNS) generation by silica in inflammation and fibrosis. Free radical biology \& medicine. 34, 1507-16.

Leung, C. C., et al., 2012. Silicosis. Lancet. 379, 2008-18.

Manning, C. B., et al., 2002. Diseases caused by asbestos: mechanisms of injury and disease development. International immunopharmacology. 2, 191-200.

Mossman, B. T., Churg, A., 1998. Mechanisms in the pathogenesis of asbestosis and silicosis. American journal of respiratory and critical care medicine. 157, 1666-80.

Mossman, B. T., et al., 2011. Pulmonary endpoints (lung carcinomas and asbestosis) following inhalation exposure to asbestos. Journal of toxicology and environmental health. Part B, Critical reviews. $14,76-121$.

Rimal, B., et al., 2005. Basic pathogenetic mechanisms in silicosis: current understanding. Current opinion in pulmonary medicine. 11, 169-73.

Robledo, R., Mossman, B., 1999. Cellular and molecular mechanisms of asbestos-induced fibrosis. Journal of cellular physiology. 180, 158-66.

Sterling, T. D., et al., 1993. Comments on the Health Effects Institute-Asbestos Research (HEI-AR) report: "Asbestos in public and commercial buildings," with emphasis on risk assessment methods used. American journal of industrial medicine. 24, 767-81.

Vallyathan, N. V., et al., 1980. Recent advances in the study of mineral pneumoconiosis. Pathology annual. $15,77-104$. 


\section{Animal models of asbestosis and silicosis}

ILSI, 2000. The relevance of the rat lung response to particle overload for human risk assessment: a workshop consensus report. ILSI Risk Science Institute Workshop Participants. Inhalation toxicology. $12,1-17$.

Bernstein, D., et al., 2005. Testing of fibrous particles: short-term assays and strategies. Inhalation toxicology. 17, 497-537.

BeruBe, K. A., et al., 1996. Comparative proliferative and histopathologic changes in rat lungs after inhalation of chrysotile or crocidolite asbestos. Toxicology and applied pharmacology. 137, 67-74.

Brass, D. M., et al., 1999. Reduced tumor necrosis factor-alpha and transforming growth factor-beta1 expression in the lungs of inbred mice that fail to develop fibroproliferative lesions consequent to asbestos exposure. The American journal of pathology. 154, 853-62.

Cummins, A. B., et al., 2003. Persistent localization of activated extracellular signal-regulated kinases (ERK1/2) is epithelial cell-specific in an inhalation model of asbestosis. The American journal of pathology. 162, 713-20.

Haegens, A., et al., 2005. Asbestos-induced lung inflammation and epithelial cell proliferation are altered in myeloperoxidase-null mice. Cancer research. 65, 9670-7.

Lasky, J. A., et al., 1998. Upregulation of the PDGF-alpha receptor precedes asbestos-induced lung fibrosis in rats. American journal of respiratory and critical care medicine. 157, 1652-7.

Manning, C. B., et al., 2008. Targeting the MEK1 cascade in lung epithelium inhibits proliferation and fibrogenesis by asbestos. American journal of respiratory cell and molecular biology. 38, 618-26.

Manning, C. B., et al., 2002. Diseases caused by asbestos: mechanisms of injury and disease development. International immunopharmacology. 2, 191-200.

\section{Cellular and Molecular Mechanisms of Asbestosis and Silicosis}

\section{Reactive oxygen and nitrogen species (ROS/RNS)}

Fenoglio, I., et al., 2003. Reaction of cysteine and glutathione (GSH) at the freshly fractured quartz surface: a possible role in silica-related diseases? Free radical biology \& medicine. 35, 752-62.

Fubini, B., Hubbard, A., 2003. Reactive oxygen species (ROS) and reactive nitrogen species (RNS) generation by silica in inflammation and fibrosis. Free radical biology \& medicine. 34, 1507-16.

Guo, W. X., et al., 1995. Effects of silica on serum phospholipid, lipid peroxide and morphological characteristics of rat lung. Biomedical and environmental sciences : BES. 8, 169-75.

Hillegass, J. M., et al., 2010. Mechanisms of oxidative stress and alterations in gene expression by Libby six-mix in human mesothelial cells. Particle and fibre toxicology. 7, 26.

Holley, J. A., et al., 1992. Increased manganese superoxide dismutase protein in type II epithelial cells of rat lungs after inhalation of crocidolite asbestos or cristobalite silica. The American journal of pathology. 141, 475-85.

Janssen, Y. M., et al., 1992. Expression of antioxidant enzymes in rat lungs after inhalation of asbestos or silica. The Journal of biological chemistry. 267, 10625-30. 
Lee, H. L., et al., 2009. Selectively decreased expression of peroxiredoxins induced by silica in pulmonary epithelial cells. The Korean journal of internal medicine. 24, 220-6.

Nagatomo, H., et al., 2007. Change of heme oxygenase-1 expression in lung injury induced by chrysotile asbestos in vivo and in vitro. Inhalation toxicology. 19, 317-23.

Sato, T., et al., 2006. Heme oxygenase-1, a potential biomarker of chronic silicosis, attenuates silicainduced lung injury. American journal of respiratory and critical care medicine. 174, 906-14.

Schins, R. P., 2002. Mechanisms of genotoxicity of particles and fibers. Inhalation toxicology. 14, 5778.

Shukla, A., et al., 2003. Multiple roles of oxidants in the pathogenesis of asbestos-induced diseases. Free radical biology \& medicine. 34, 1117-29.

Vallyathan, V., et al., 1995. Freshly fractured quartz inhalation leads to enhanced lung injury and inflammation. Potential role of free radicals. American journal of respiratory and critical care medicine. 152, 1003-9.

\section{Importance of inflammatory cell types}

Barbarin, V., et al., 2004. Characterization of the effect of interleukin-10 on silica-induced lung fibrosis in mice. American journal of respiratory cell and molecular biology. 31, 78-85.

Brown, J. M., et al., 2007. Silica-directed mast cell activation is enhanced by scavenger receptors. American journal of respiratory cell and molecular biology. 36, 43-52.

Cassel, S. L., et al., 2008. The Nalp3 inflammasome is essential for the development of silicosis. Proceedings of the National Academy of Sciences of the United States of America. 105, 9035-40.

Davis, G. S., et al., 1998. Persistent overexpression of interleukin-1beta and tumor necrosis factoralpha in murine silicosis. Journal of environmental pathology, toxicology and oncology : official organ of the International Society for Environmental Toxicology and Cancer. 17, 99-114.

Davis, G. S., et al., 2006. Interleukin-12 is not essential for silicosis in mice. Particle and fibre toxicology. 3,2 .

Driscoll, K. E., 2000. TNFalpha and MIP-2: role in particle-induced inflammation and regulation by oxidative stress. Toxicology letters. 112-113, 177-83.

Driscoll, K. E., et al., 1993. Macrophage inflammatory proteins 1 and 2: expression by rat alveolar macrophages, fibroblasts, and epithelial cells and in rat lung after mineral dust exposure. American journal of respiratory cell and molecular biology. 8, 311-8.

Haberzettl, P., et al., 2008. Impact of the FcgammaII-receptor on quartz uptake and inflammatory response by alveolar macrophages. American journal of physiology. Lung cellular and molecular physiology. 294, L1137-48.

Haegens, A., et al., 2007. Airway epithelial NF-kappaB activation modulates asbestos-induced inflammation and mucin production in vivo. Journal of immunology. 178, 1800-8.

Hamilton, R. F., Jr., et al., 2006. MARCO mediates silica uptake and toxicity in alveolar macrophages from C57BL/6 mice. The Journal of biological chemistry. 281, 34218-26. 
Kokkinis, F. P., et al., 2011. Bronchoalveolar lavage fluid cellular profile in workers exposed to chrysotile asbestos. Toxicology and industrial health. 27, 849-56.

Maeda, M., et al., 2011. Decreased CXCR3 expression in CD4+ T cells exposed to asbestos or derived from asbestos-exposed patients. American journal of respiratory cell and molecular biology. 45, 795803.

Olbruck, H., et al., 1998. Supernatants from quartz dust treated human macrophages stimulate cell proliferation of different human lung cells as well as collagen-synthesis of human diploid lung fibroblasts in vitro. Toxicology letters. 96-97, 85-95.

Piguet, P. F., 1993. Cytokines involved in pulmonary fibrosis. International review of experimental pathology. 34 Pt B, 173-81.

Song, L., et al., 2012. Tregs promote the differentiation of Th17 cells in silica-induced lung fibrosis in mice. PloS one. 7, e37286.

Suzuki, N., et al., 1993. Mast cells are essential for the full development of silica-induced pulmonary inflammation: a study with mast cell-deficient mice. American journal of respiratory cell and molecular biology. 9, 475-83.

Thakur, S. A., et al., 2008. Role of scavenger receptor a family in lung inflammation from exposure to environmental particles. Journal of immunotoxicology. 5, 151-7.

\section{The lung epithelium, a key player in fibrogenesis}

Albrecht, C., et al., 2001. Clara-cell hyperplasia after quartz and coal-dust instillation in rat lung. Inhalation toxicology. 13, 191-205.

Bardales, R. H., et al., 1996. Apoptosis is a major pathway responsible for the resolution of type II pneumocytes in acute lung injury. The American journal of pathology. 149, 845-52.

Churg, A., 1996. The uptake of mineral particles by pulmonary epithelial cells. American journal of respiratory and critical care medicine. $154,1124-40$.

Dixon, D., et al., 1995. Incorporation of bromodeoxyuridine (BrdU) in the bronchiolar-alveolar regions of the lungs following two inhalation exposures to chrysotile asbestos in strain $\mathrm{A} / \mathrm{J}$ mice. Journal of environmental pathology, toxicology and oncology : official organ of the International Society for Environmental Toxicology and Cancer. 14, 205-13.

Driscoll, K. E., et al., 1998. Crocidolite activates NF-kappa B and MIP-2 gene expression in rat alveolar epithelial cells. Role of mitochondrial-derived oxidants. Environmental health perspectives. 106 Suppl 5, 1171-4.

Driscoll, K. E., et al., 1993. Macrophage inflammatory proteins 1 and 2: expression by rat alveolar macrophages, fibroblasts, and epithelial cells and in rat lung after mineral dust exposure. American journal of respiratory cell and molecular biology. 8, 311-8.

Driscoll, K. E., et al., 1996. Alpha-quartz-induced chemokine expression by rat lung epithelial cells: effects of in vivo and in vitro particle exposure. The American journal of pathology. 149, 1627-37.

Gwinn, M. R., et al., 2009. The role of p53 in silica-induced cellular and molecular responses associated with carcinogenesis. Journal of toxicology and environmental health. Part A. 72, 1509-19. 
Heintz, N. H., et al., 2010. Asbestos, lung cancers, and mesotheliomas: from molecular approaches to targeting tumor survival pathways. American journal of respiratory cell and molecular biology. 42, 133-9.

Herseth, J. I., et al., 2008. IL-1beta differently involved in IL-8 and FGF-2 release in crystalline silicatreated lung cell co-cultures. Particle and fibre toxicology. 5, 16.

Kamp, D. W., et al., 1995. Asbestos causes DNA strand breaks in cultured pulmonary epithelial cells: role of iron-catalyzed free radicals. The American journal of physiology. 268, L471-80.

Lesur, O., et al., 1995. Alterations of surfactant lipid turnover in silicosis: evidence of a role for surfactant-associated protein A (SP-A). International journal of experimental pathology. 76, 287-98.

Liu, G., et al., 2010. Molecular mechanisms of asbestos-induced lung epithelial cell apoptosis. Chemico-biological interactions. 188, 309-18.

Melloni, B., et al., 1994. Partial characterization of the proliferative activity for fetal lung epithelial cells produced by silica-exposed alveolar macrophages. Journal of leukocyte biology. 55, 574-80.

Melloni, B., et al., 1996. Effect of exposure to silica on human alveolar macrophages in supporting growth activity in type II epithelial cells. Thorax. 51, 781-6.

Mossman, B. T., et al., 2006. Oxidants and signaling by mitogen-activated protein kinases in lung epithelium. American journal of respiratory cell and molecular biology. 34, 666-9.

Olbruck, H., et al., 1998. Supernatants from quartz dust treated human macrophages stimulate cell proliferation of different human lung cells as well as collagen-synthesis of human diploid lung fibroblasts in vitro. Toxicology letters. 96-97, 85-95.

Ovrevik, J., et al., 2009. Cytokine and chemokine expression patterns in lung epithelial cells exposed to components characteristic of particulate air pollution. Toxicology. 259, 46-53.

Perkins, T. N., et al., 2012. Differences in gene expression and cytokine production by crystalline vs. amorphous silica in human lung epithelial cells. Particle and fibre toxicology. 9, 6.

\section{Fibroblasts as effector and target cells in fibrosis}

Absher, M., et al., 1993. Patterns of secretion of transforming growth factor-alpha (TGF-alpha) in experimental silicosis. Acute and subacute effects of cristobalite exposure in the rat. Regional immunology. 5, 225-31.

Chen, F., et al., 1994. Excessive production of insulin-like growth factor-I by silicotic rat alveolar macrophages. APMIS: acta pathologica, microbiologica, et immunologica Scandinavica. 102, 581-8.

Driscoll, K. E., 2000. TNFalpha and MIP-2: role in particle-induced inflammation and regulation by oxidative stress. Toxicology letters. 112-113, 177-83.

Gardi, C., et al., 1997. Iron mobilization from crocidolite as enhancer of collagen content in rat lung fibroblasts. Biochemical pharmacology. 53, 1659-65.

Jagirdar, J., et al., 1996. Transforming growth factor-beta (TGF-beta) in silicosis. American journal of respiratory and critical care medicine. 154, 1076-81.

Kopnin, P. B., et al., 2004. Cell type-specific effects of asbestos on intracellular ROS levels, DNA oxidation and G1 cell cycle checkpoint. Oncogene. 23, 8834-40. 
Kumar, R. K., et al., 1994. Epidermal growth factor-like activity in bronchoalveolar lavage fluid in experimental silicosis. Growth factors. 10, 163-70.

Lasky, J. A., et al., 1995. Chrysotile asbestos stimulates platelet-derived growth factor-AA production by rat lung fibroblasts in vitro: evidence for an autocrine loop. American journal of respiratory cell and molecular biology. 12, 162-70.

Mariani, T. J., et al., 1996. Localization of type I procollagen gene expression in silica-induced granulomatous lung disease and implication of transforming growth factor-beta as a mediator of fibrosis. The American journal of pathology. 148, 151-64.

Partanen, R., et al., 1994. The detection of increased amounts of the extracellular domain of the epidermal growth factor receptor in serum during carcinogenesis in asbestosis patients. Journal of occupational medicine. : official publication of the Industrial Medical Association. 36, 1324-8.

Perdue, T. D., Brody, A. R., 1994. Distribution of transforming growth factor-beta 1, fibronectin, and smooth muscle actin in asbestos-induced pulmonary fibrosis in rats. The journal of histochemistry and cytochemistry: official journal of the Histochemistry Society. 42, 1061-70.

Williams, A. O., et al., 1993. Immunohistochemical localization of transforming growth factor-beta 1 in rats with experimental silicosis, alveolar type II hyperplasia, and lung cancer. The American journal of pathology. 142, 1831-40.

Zhang, Y., et al., 1993. Enhanced IL-1 beta and tumor necrosis factor-alpha release and messenger RNA expression in macrophages from idiopathic pulmonary fibrosis or after asbestos exposure. Journal of immunology. 150, 4188-96.

\section{Dissolution and deposition of extracellular matrix (ECM)}

Absher, M. P., et al., 1989. Biphasic cellular and tissue response of rat lungs after eight-day aerosol exposure to the silicon dioxide cristobalite. The American journal of pathology. 134, 1243-51.

Churg, A., Wright, J. L., 2002. Airway wall remodeling induced by occupational mineral dusts and air pollutant particles. Chest. 122, 306S-309S.

Dai, J., Churg, A., 2001. Relationship of fiber surface iron and active oxygen species to expression of procollagen, PDGF-A, and TGF-beta(1) in tracheal explants exposed to amosite asbestos. American journal of respiratory cell and molecular biology. 24, 427-35.

Dai, J., et al., 1998. Mineral dusts directly induce epithelial and interstitial fibrogenic mediators and matrix components in the airway wall. American journal of respiratory and critical care medicine. 158, 1907-13.

Delgado, L., et al., 2006. Apoptosis and extracellular matrix remodelling in human silicosis. Histopathology. 49, 283-9.

Kroczynska, B., et al., 2006. Crocidolite asbestos and SV40 are cocarcinogens in human mesothelial cells and in causing mesothelioma in hamsters. Proceedings of the National Academy of Sciences of the United States of America. 103, 14128-33.

Langley, R. J., et al., 2011. Fibrogenic and redox-related but not proinflammatory genes are upregulated in Lewis rat model of chronic silicosis. Journal of toxicology and environmental health. Part A. 74, 1261-79. 
Li, J., et al., 2004. Effect of platelet-derived growth factor on the development and persistence of asbestos-induced fibroproliferative lung disease. Journal of environmental pathology, toxicology and oncology : official organ of the International Society for Environmental Toxicology and Cancer. 23, $253-66$.

Li, K., et al., 1996. Mineral dusts cause elastin and collagen breakdown in the rat lung: a potential mechanism of dust-induced emphysema. American journal of respiratory and critical care medicine. $153,644-9$.

Mariani, T. J., et al., 1999. Fibroblast tropoelastin and alpha-smooth-muscle actin expression are repressed by particulate-activated macrophage-derived tumor necrosis factor-alpha in experimental silicosis. American journal of respiratory cell and molecular biology. 21, 185-92.

Morimoto, Y., et al., 1997. Expression of matrix metalloproteinases, tissue inhibitors of metalloproteinases, and extracellular matrix mRNA following exposure to mineral fibers and cigarette smoke in vivo. Environmental health perspectives. 105 Suppl 5, 1247-51.

Mutsaers, S. E., et al., 1998. Fibroblast mitogens in bronchoalveolar lavage (BAL) fluid from asbestos-exposed subjects with and without clinical evidence of asbestosis: no evidence for the role of PDGF, TNF-alpha, IGF-1, or IL-1 beta. The Journal of pathology. 185, 199-203.

Perez-Ramos, J., et al., 1999. Matrix metalloproteinases 2, 9, and 13, and tissue inhibitors of metalloproteinases 1 and 2 in experimental lung silicosis. American journal of respiratory and critical care medicine. 160, 1274-82.

Scabilloni, J. F., et al., 2005. Matrix metalloproteinase induction in fibrosis and fibrotic nodule formation due to silica inhalation. American journal of physiology. Lung cellular and molecular physiology. 288, L709-17.

Shukla, A., et al., 2006. Transcriptional up-regulation of MMP12 and MMP13 by asbestos occurs via a PKCdelta-dependent pathway in murine lung. FASEB journal : official publication of the Federation of American Societies for Experimental Biology. 20, 997-9.

Tan, R. J., et al., 2006a. Matrix metalloproteinases promote inflammation and fibrosis in asbestosinduced lung injury in mice. American journal of respiratory cell and molecular biology. 35, 289-97.

Tan, R. J., et al., 2006b. Inflammatory cells as a source of airspace extracellular superoxide dismutase after pulmonary injury. American journal of respiratory cell and molecular biology. 34, 226-32.

Yee, H., et al., 2008. Immunohistochemical study of fibrosis and adenocarcinoma in dominantnegative p53 transgenic mice exposed to chrysotile asbestos and benzo(a)pyrene. Journal of environmental pathology, toxicology and oncology: official organ of the International Society for Environmental Toxicology and Cancer. 27, 267-76.

\section{Cell signaling in lung Inflammation and fibrogenesis}

Buder-Hoffmann, S., et al., 2001. Different accumulation of activated extracellular signal-regulated kinases (ERK 1/2) and role in cell-cycle alterations by epidermal growth factor, hydrogen peroxide, or asbestos in pulmonary epithelial cells. American journal of respiratory cell and molecular biology. 24, 405-13.

Castranova, V., 2004. Signaling pathways controlling the production of inflammatory mediators in response to crystalline silica exposure: role of reactive oxygen/nitrogen species. Free radical biology \& medicine. 37, 916-25. 
Cummins, A. B., et al., 2003. Persistent localization of activated extracellular signal-regulated kinases (ERK1/2) is epithelial cell-specific in an inhalation model of asbestosis. The American journal of pathology. 162, 713-20.

Ding, M., et al., 2002. Diseases caused by silica: mechanisms of injury and disease development. International immunopharmacology. 2, 173-82.

Driscoll, K. E., et al., 1998. Crocidolite activates NF-kappa B and MIP-2 gene expression in rat alveolar epithelial cells. Role of mitochondrial-derived oxidants. Environmental health perspectives. 106 Suppl 5, 1171-4.

Haegens, A., et al., 2007. Airway epithelial NF-kappaB activation modulates asbestos-induced inflammation and mucin production in vivo. Journal of immunology. 178, 1800-8.

Heintz, N. H., et al., 1993. Persistent induction of c-fos and c-jun expression by asbestos. Proceedings of the National Academy of Sciences of the United States of America. 90, 3299-303.

Lehnert, B. E. (1993). Defense mechanisms against inhaled particles and associated particle-cell interactions. In G. D. Guthrie, Jr. and B. T. Mossman, editors. Health Effects of Mineral Dusts. Reviews in Mineralogy, Vol. 28. Mineralogical Society of America, Washington, DC. 427-470.

Manning, C. B., et al., 2002. Diseases caused by asbestos: mechanisms of injury and disease development. International immunopharmacology. 2, 191-200.

Ovrevik, J., et al., 2004. p38 and Src-ERK1/2 pathways regulate crystalline silica-induced chemokine release in pulmonary epithelial cells. Toxicological sciences : an official journal of the Society of Toxicology. 81, 480-90.

Partanen, R., et al., 1994. The detection of increased amounts of the extracellular domain of the epidermal growth factor receptor in serum during carcinogenesis in asbestosis patients. Journal of occupational medicine. : official publication of the Industrial Medical Association. 36, 1324-8.

Piguet, P. F., Vesin, C., 1994. Treatment by human recombinant soluble TNF receptor of pulmonary fibrosis induced by bleomycin or silica in mice. The European respiratory journal : official journal of the European Society for Clinical Respiratory Physiology. 7, 515-8.

Piguet, P. F., et al., 1993. Interleukin 1 receptor antagonist (IL-1ra) prevents or cures pulmonary fibrosis elicited in mice by bleomycin or silica. Cytokine. 5, 57-61.

Ramos-Nino, M. E., et al., 2002. Role of mitogen-activated protein kinases (MAPK) in cell injury and proliferation by environmental particulates. Molecular and cellular biochemistry. 234-235, 111-8.

Robledo, R., Mossman, B., 1999. Cellular and molecular mechanisms of asbestos-induced fibrosis. Journal of cellular physiology. 180, 158-66.

Robledo, R. F., et al., 2000. Increased phosphorylated extracellular signal-regulated kinase immunoreactivity associated with proliferative and morphologic lung alterations after chrysotile asbestos inhalation in mice. The American journal of pathology. 156, 1307-16.

Scapoli, L., et al., 2004. Src-dependent ERK5 and Src/EGFR-dependent ERK1/2 activation is required for cell proliferation by asbestos. Oncogene. 23, 805-13. 
Shukla, A., et al., 2007. Asbestos-induced peribronchiolar cell proliferation and cytokine production are attenuated in lungs of protein kinase C-delta knockout mice. The American journal of pathology. $170,140-51$.

Shukla, A., et al., 2003a. Cell signaling and transcription factor activation by asbestos in lung injury and disease. The international journal of biochemistry \& cell biology. 35, 1198-209.

Shukla, A., et al., 2003b. Asbestos-induced apoptosis is protein kinase C delta-dependent. American journal of respiratory cell and molecular biology. 29, 198-205.

Timblin, C. R., et al., 1995. Transcriptional activation of the proto-oncogene c-jun by asbestos and $\mathrm{H} 2 \mathrm{O} 2$ is directly related to increased proliferation and transformation of tracheal epithelial cells. Cancer research. 55, 2723-6. 



\title{
CHAPTER 3
}

\section{INDICATIONS FOR DISTINC PATHOGENIC MECHANISMS OF ASBESTOS AND SILICA THROUGH GENE EXPRESSION PROFILING OF LUNG EPITHELIAL CELLS}

\author{
Timothy N Perkins ${ }^{1,2} *$, Paul M Peeters ${ }^{1,2} *$, Arti Shukla ${ }^{1}$, Ingrid Arijs ${ }^{3,4}$, Julie Dragon ${ }^{5}$, Emiel \\ FM Wouters ${ }^{2}$, Niki L Reynaert ${ }^{2}$, Brooke $\mathrm{T} \operatorname{Mossman}^{1}(*=$ equally contributing first \\ authors)

\footnotetext{
${ }^{1}$ Department of Pathology, University of Vermont College of Medicine, 89 Beaumont Avenue, Burlington, VT 05405, USA

${ }^{2}$ Department of Respiratory Medicine, Maastricht University Medical Centre, Maastricht University, Maastricht, The Netherlands.

${ }^{3}$ Department of Gastroenterology, Translational Research Center for Gastrointestinal Disorders (TARGID), and ${ }^{4}$ Gene Expression Unit, Department of Molecular Cell Biology, KU Leuven, Belgium

${ }^{5}$ Department of Microbiology and Molecular Genetics, University of Vermont, 95 Carrigan Drive, Burlington, VT 05405, USA
}

Hum Mol Genet. 2015 Mar 1;24(5):1374-89.

\begin{abstract}
Occupational and environmental exposures to airborne asbestos and silica are associated with the development of lung fibrosis in the forms of asbestosis and silicosis, respectively. However, both diseases display distinct pathological presentations, likely associated with differences in gene expression induced by different mineral structures, composition and biopersistent properties. We hypothesized that effects of mineral exposure in the airway epithelium may dictate deviating molecular events that may explain the different pathologies of asbestosis vs. silicosis. Using robust gene expression-profiling in conjunction with in-depth pathway analysis, we assessed early $(24 \mathrm{~h})$ alterations in gene expression associated with crocidolite asbestos or cristobalite silica exposures in primary human bronchial epithelial cells (NHBE). Observations were confirmed in an immortal human bronchial epithelial cell line (BEAS-2B) by QRT-PCR and protein assays. Utilization of overall gene expression, unsupervised hierarchical cluster analysis and integrated pathway analysis revealed gene alterations that were common to both minerals or unique to either mineral. Our findings reveal that both minerals had potent effects on genes governing cell adhesion/migration, inflammation, and cellular stress, key features of fibrosis. Asbestos exposure was most specifically associated with aberrant cell proliferation and carcinogenesis, whereas silica exposure was highly associated with additional inflammatory responses, as well as pattern recognition, and fibrogenesis. These findings illustrate the use of gene-profiling as a means to determine early molecular events that may dictate pathological processes induced by exogenous cellular insults. In addition, it is a useful approach for predicting the pathogenicity of potentially harmful materials.
\end{abstract}





\section{BACKGROUND}

Asbestosis and silicosis, the most common forms of pneumoconioses, are caused by inhalation of respirable asbestos fibers and crystalline silica particles, respectively. 'Asbestos' describes a group of silicate minerals comprised of serpentine fibers (i.e. chrysotile) and rigid, highly pathogenic amphibole fibers (e.g. crocidolite, amosite, tremolite, actinolite, anthophyllite). Inhalation of asbestos causes major changes in parenchymal architecture, including diffuse interstitial collagen deposition and accumulation of dense connective tissue at specific loci (1-3). In addition, amphibole asbestos causes pleural fibrosis, and results in lung cancer and malignant mesotheliomas (3). The three naturally occurring polymorphs of crystalline silica of health concern are quartz, cristobalite and tridymite (2). Unlike asbestosis, silicosis is associated with the formation of concentric "whorled" nodules of collagen-rich, dense fibrotic tissue and a prominent granulomatous response $(1,3)$. Occupational exposures to silica, especially in smokers, have been associated with increased risks of lung cancer, but not mesotheliomas, in some cohorts, although this is a topic of debate (4-7).

Impingement of high concentrations of both fibers and particles on alveolar macrophages and epithelial cells lining the upper and lower respiratory tract, is characterized by cell damage associated with the production of oxidants, a persistent inflammatory response, the generation of profibrotic mediators, and scarring (8). Although exposure to each type of mineral can give rise to interstitial fibrosis, physicochemical differences between crystalline silica particles and asbestos fibers may lead to distinctive gene expression patterns resulting in the dissimilar pathological characteristics of silicosis and asbestosis. The role of the lung epithelium has until recently had been viewed as a passive, physical barrier against inhaled particulates and other toxicants. However, studies show that lung epithelial cells play an active role in particulate uptake $(9,10)$, immune cell recruitment $(3)$, proliferation of epithelial and other cell types (11), and matrix remodeling (12). Since epithelial cells are target cells of lung carcinomas and contribute to the development of pulmonary fibrosis, understanding the molecular responses of human lung epithelial cells to pathogenic minerals is critical to unraveling the complex molecular events that initiate inflammatory responses, consequent fibrogenesis and other mechanisms intrinsic to these diseases. In this study, we therefore compared gene alterations induced by exposure to equitoxic doses of crocidolite asbestos and cristobalite silica in an isolate of normal human bronchial epithelial cells (NHBE) (9). By gene-profiling, we furthermore explored expression signatures and their biological interactions. Selected common as well as mineral-specific gene alterations were confirmed in an immortalized bronchial epithelial cell line (BEAS-2B) by QRT-PCR. Additionally, further confirmation of selected findings at the protein level was obtained using various techniques. To our knowledge, these studies are the first to explore comparative gene expression profiling in human airway epithelial cells using equitoxic doses of pathogenic minerals, i.e. an amphibole asbestos and a crystalline silica. 


\section{RESULTS}

\section{Effects of mineral exposure on NHBE and BEAS-2B cell viability}

We initially compared the effects of crocidolite asbestos and cristobalite silica on cell viability using surface area-based dosimetry, as the biological activity of these particulates is derived largely from their surface reactivity (13). Based on data from these experiments, a concentration of $15 \times 10^{6} \mu^{2} / \mathrm{cm}^{2}$ dish was chosen for asbestos, and 15 and $75 \times 10^{6}$ $\mu \mathrm{m}^{2} / \mathrm{cm}^{2}$ dish for silica for NHBE gene expression studies $\left(<\mathrm{LD}_{50}\right.$ concentrations) (Supplemental material, Figure S1). The viability of BEAS-2B cells exposed to minerals for $24 \mathrm{~h}$ was determined similarly (Supplemental material, Figure S2). As cells from the immortalized cell line were more resistant to equal surface-area based doses as compared to primary cell cultures, mineral concentrations of 75 and $150 \times 10^{6} \mu \mathrm{m}^{2} / \mathrm{cm}^{2}$ dish were used for gene expression studies in BEAS-2B cells $(9,14)$.

\section{Strategies to detect gene expression alterations by minerals}

Using equitoxic doses noted above, gene expression was assessed by Affymetrix GeneChip arrays analyzed with GeneSifter ${ }^{\circledR}$ and Bioconductor. An exposure period of $24 \mathrm{~h}$ was used as this is the time-point for maximal gene expression changes by a number of pathogenic fibers and particles $(9,10,15,16)$. In our previous studies, non-pathogenic glass beads and fine $\mathrm{TiO}_{2}$ did not induce any significant numbers of gene changes compared to unexposed cells $(10,17)$, which were therefore used as controls here. First, to visualize the gene-sample relationship, an unsupervised average-linkage hierarchical clustering was applied to the 50 probe-sets with the highest variation across the 12 samples. This analysis clearly identified distinct clusters related to different minerals and doses (Figure 1A). Cluster I was comprised of two subclusters: Ia including the unexposed control samples and the low dose $\left(15 \mathrm{X} 10^{6}\right.$ $\left.\mu \mathrm{m}^{2} / \mathrm{cm}^{2}\right)$ silica group, and $\mathrm{Ib}$ containing the asbestos $\left(15 \mathrm{X} 10^{6} \mu \mathrm{m}^{2} / \mathrm{cm}^{2}\right)$ samples. Cluster II contained the high dose $\left(75 \times 10^{6} \mu \mathrm{m}^{2} / \mathrm{cm}^{2}\right)$ silica samples.

To compare the magnitude of alterations in gene expression by each mineral and dose, we examined the total number of significant gene changes $>1.5$-fold vs. unexposed controls, with an adj. p-value $<0.05(\mathrm{FDR}<0.05)$. Asbestos $\left(15 \times 10^{6} \mathrm{~mm}^{2} / \mathrm{cm}^{2}\right)$ altered levels of 321 probesets (262 genes) which was much more robust than silica, which altered only 3 probe-sets (3 genes) at an equal concentration $\left(15 \times 10^{6} \mu \mathrm{m}^{2} / \mathrm{cm}^{2}\right)$. However, at the higher amount, silica $\left(75 \times 10^{6} \mu^{2} / \mathrm{cm}^{2}\right)$ exposures showed significant changes in 1247 probe-sets (1033 genes) (Table 1, Figure 1B). A comprehensive list of significant gene changes for each group is described in supplemental materials (Supplemental Tables 1-3). 

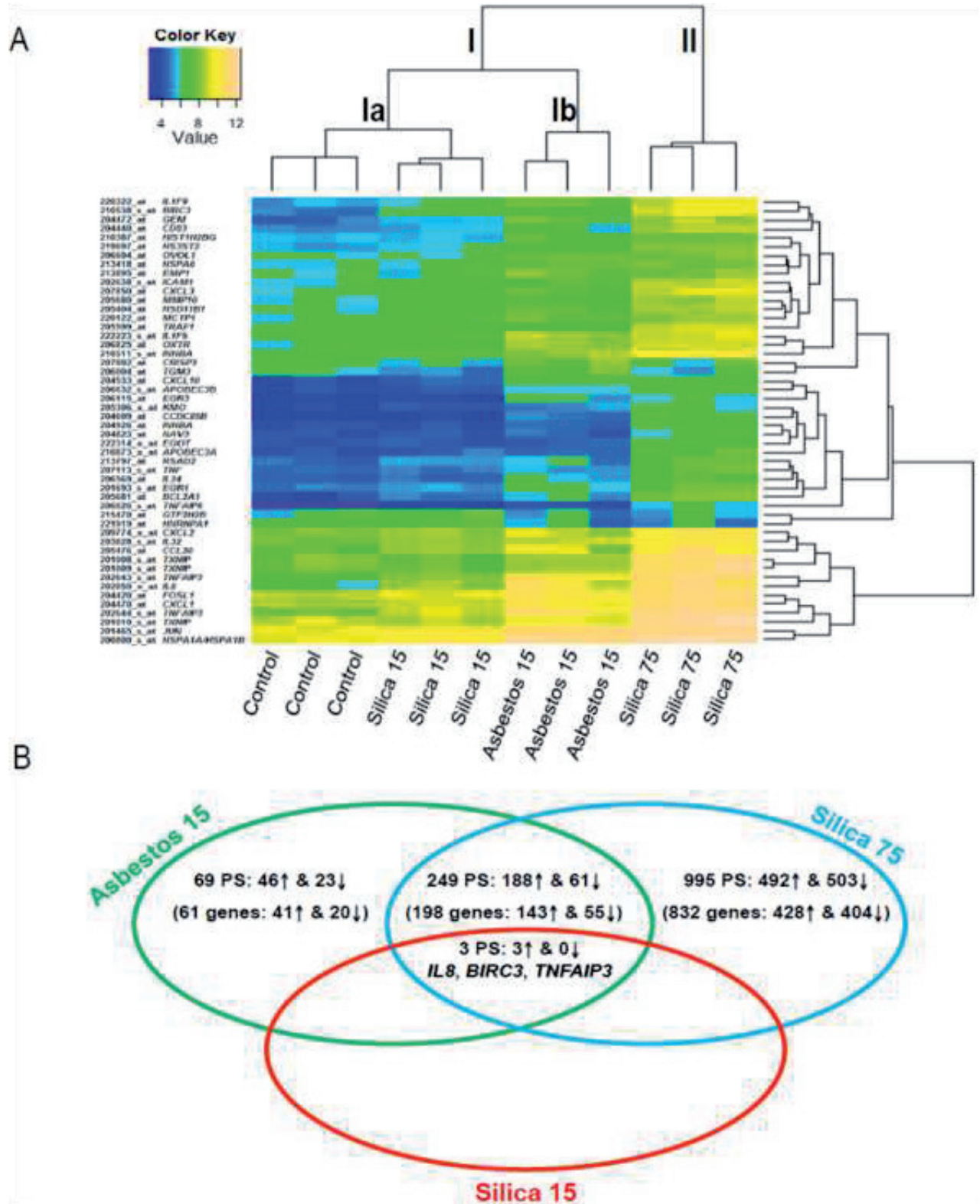

Figure 1: Unsupervised hierarchical cluster analysis and overall gene changes in mineral exposed NHBE cells.

(A) Unsupervised hierarchical cluster analysis of gene expression profiles of unexposed control NHBE cells and cells exposed to crocidolite asbestos $15 \times 10^{6} \mathrm{\mu m}^{2} / \mathrm{cm}^{2}$ (Asbestos 15), cristobalite silica $15 \times 10^{6} \mathrm{\mu m}^{2} / \mathrm{cm}^{2}$ (Silica 15), or $75 \times 10^{6} \mathrm{\mu m}^{2} / \mathrm{cm}^{2}$ (Silica 75) $(N=3$ samples/group) based on the $\log _{2}$ expression values of the top 50 most variable probe-sets. Individual samples are shown in columns and genes in rows. The $\log _{2}$ expression values for individual genes are indicated by color (yellow indicates a high and blue a low level of expression). (B) Venn diagram representation of the 
total number of probe-sets and genes (up-regulated and down-regulated) significantly altered by all 3 exposure groups compared. This includes those specific to each exposure as well as those commonly affected by different exposures [ $\geq 1.5$-fold vs. controls; adj. $p<0.05(F D R<0.05)]$.

Table 1: Total number of gene alterations in NHBE cells $(24 \mathrm{~h})$. Total numbers of probe-sets, and total numbers of genes significantly altered by each mineral and dose compared to unexposed controls. A threshold cut-off of 1.5-fold vs. controls with an adj.p-value $<0.05$ was used.

\begin{tabular}{lllll}
\hline Mineral & Dose (S.A.) & Dose (mass) & Probe-sets & Genes \\
\hline Crocidolite Asbestos & $15 \times 10^{6} \mu \mathrm{m}^{2} / \mathrm{cm}^{2}$ & $1.0 \mu \mathrm{g} / \mathrm{cm}^{2}$ & 321 & 262 \\
Cristobalite Silica & $15 \times 10^{6} \mu \mathrm{m}^{2} / \mathrm{cm}^{2}$ & $2.94 \mu \mathrm{g} / \mathrm{cm}^{2}$ & 3 & 3 \\
Cristobalite Silica & $75 \times 10^{6} \mu \mathrm{m}^{2} / \mathrm{cm}^{2}$ & $14.7 \mu \mathrm{g} / \mathrm{cm}^{2}$ & 1247 & 1033 \\
\hline
\end{tabular}

\section{Comparative gene expression and divergent signaling pathways induced by asbestos and silica}

To determine both common and divergent gene expression patterns and signaling pathways associated with mineral-induced gene expression changes, IPA ${ }^{\circledR}$ was used. Comparative analysis revealed that of 1316 probe-sets significantly altered across all three exposures (compared to controls), 252 were commonly altered by asbestos $\left(15 \times 10^{6} \mu^{2} / \mathrm{cm}^{2}\right)$ and silica $\left(75 \times 10^{6} \mu \mathrm{m}^{2} / \mathrm{cm}^{2}\right.$ ) (Supplemental Table 4), 3 of which were also significantly altered by the low dose silica $\left(15 \times 10^{6} \mathrm{\mu m}^{2} / \mathrm{cm}^{2}\right)$ (Figure 1B). Conversely, 69 probe-sets were specifically altered by asbestos $\left(15 \times 10^{6} \mu \mathrm{m}^{2} / \mathrm{cm}^{2}\right)$ (Supplemental Table 5) and 995 by silica $\left(75 \times 10^{6} \mu^{2} / \mathrm{cm}^{2}\right.$ ) (Supplemental Table 6). These gene changes were analyzed to determine signaling pathways, biological, and disease functions associated with mineral exposures. The top ten canonical pathways and genes associated with these pathways were used to construct schematic representations of the major signaling events affected commonly or specifically by asbestos- or silica (Figure 2, 5, 7; Tables 2-4).

\section{Common responses}

Canonical pathways affected commonly by both minerals were associated with cell membrane receptors and down-stream signaling [e.g. Caspase-1 (CASP1)], transcriptional regulators [i.e. Early growth response (EGR) and Activator protein-1 (AP-1), and Nuclear factor kappa B $(\mathrm{NF}-\kappa \mathrm{B})]$, and down-stream biological functions. These biological functions included extracellular matrix (ECM) dissolution, leukocyte adhesion and migration, inflammation, fibrotic response, apoptosis regulation, and cellular stress responses, as well as other biological activities such as mitogen-activated protein kinase (MAPK) inhibition and glucocorticoid signaling (Table 2, Figure 2). It should be noted that all gene symbols are defined in Supplemental material, Supplemental Tables 4-6. The up-regulation of interleukin-8 (IL8), matrix metallopeptidase 1 (MMP1), and baculoviral IAP-repeatcontaining 3 (BIRC3) by both asbestos and silica exposures observed by microarray was confirmed in NHBE cells by QRT-PCR in a separate experiment (Supplemental material, Figure S3). These findings were also confirmed in BEAS-2B cells (Figure 3A-C). Common up-regulation of the early-response gene, JUN, that encodes a member of the AP-1 transcription factor complex (Figure 3D), and is known to have a pivotal role in asbestos- and silica-induced disease processes (18-20) was confirmed in BEAS-2B cells. In addition, up- 
regulation of a number of novel genes by both asbestos and silica exposure was confirmed in BEAS-2B cells including the transcriptional regulators EGR1 and TNFAIP3 (Figure 3E and F), the inflammatory receptor IL1RL1 (Figure 3G), the apoptosis inducer IL24 (Figure 3H), and the cell stress response protein HSPA6 (Figure 3I).

Additionally, some common changes observed at the mRNA level were investigated at the protein level in BEAS-2B cells (Figure 4). In addition to the increased mRNA levels of CASP1 observed for both minerals in NHBE cells (Figure 2), the enzymatic activity levels of this effector molecule of the NLRP3 inflammasome were found to be increased in BEAS2B exposed to asbestos and silica for $24 \mathrm{~h}$ (Figure 4A). Furthermore, we confirm that asbestos and silica both cause secretion of IL-8 (Figure 4B), although comparing equal surface-area based doses, asbestos induced higher levels of secreted IL-8 which is in agreement with our mRNA data (Figure 3A). Moreover, both fibers and particles caused a marked increase in the protein expression of CLDN1 (Figure 4C), a tight junction protein important in supporting the epithelium as a physical barrier and in regulating the inflammatory response $(21,22)$.

Table 2: Top 10 canonical pathways altered by both asbestos and silica in NHBE (24 h). Top 10 canonical pathways significantly enriched by alteration in gene expression by both asbestos and silica as determined by core analysis of commonly altered gene changes in IPA.

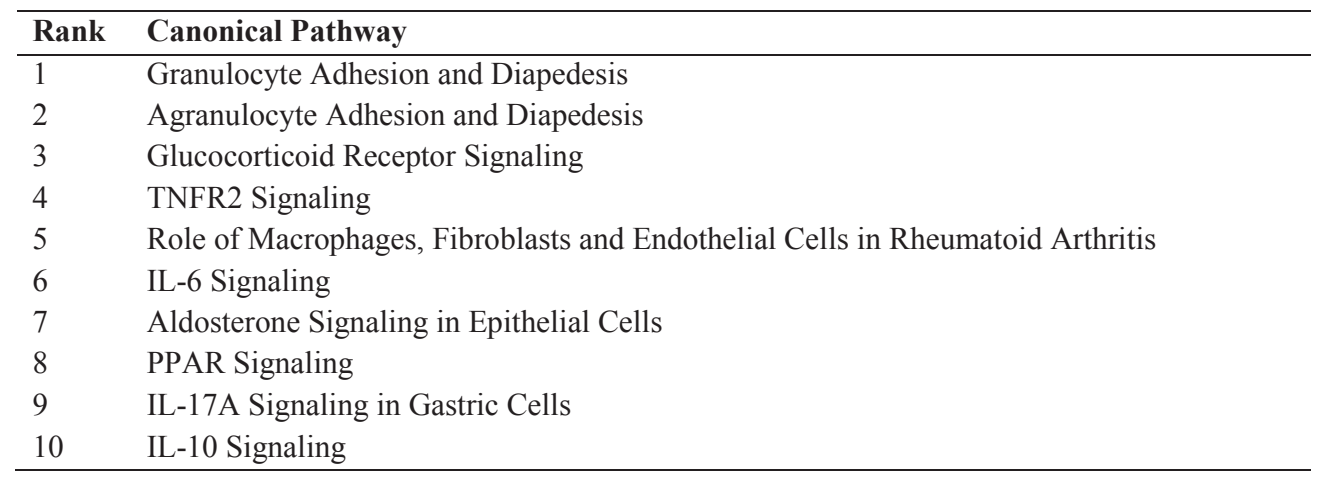




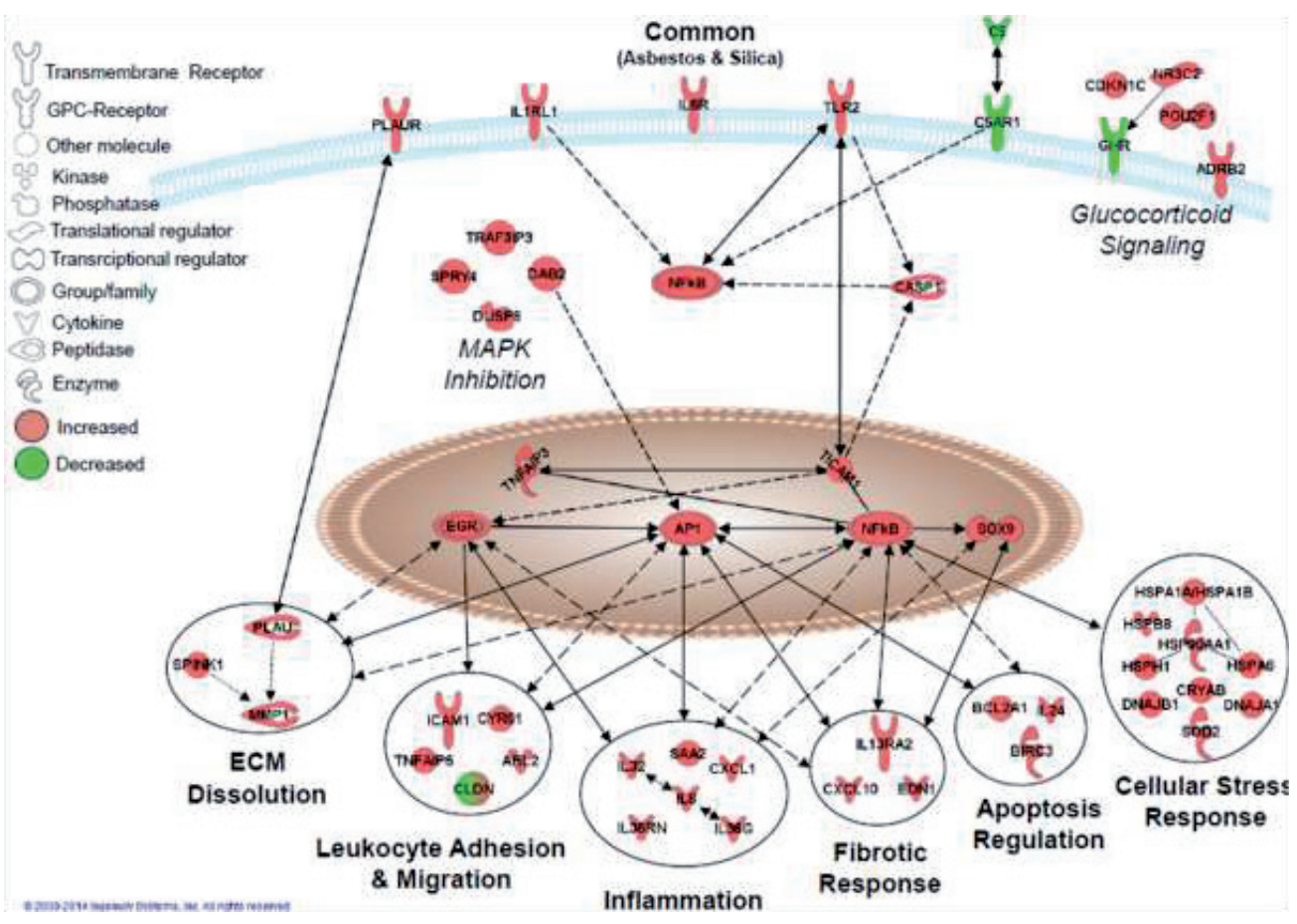

Figure 2: Pathways commonly affected by asbestos and silica in NHBE cells. A schematic representation of 38 genes with altered expression by asbestos $\left(15 \times 10^{6} \mu^{2} / \mathrm{cm}^{2}\right)$ and silica $\left(75 \times 10^{6} \mathrm{\mu m}^{2} / \mathrm{cm}^{2}\right)$ at $24 \mathrm{~h}$. Red: up-regulated, green: down-regulated [ $\geq 1.5$-fold vs. controls; adj. $p<0.05(F D R<0.05)]$. Lines and arrows interconnecting molecules represent associations determined by IPA from the Ingenuity Knowledge Base, including both direct and indirect relationships. All gene names, fold-change values, and adj.p-values are listed in Supplemental Table 4. 

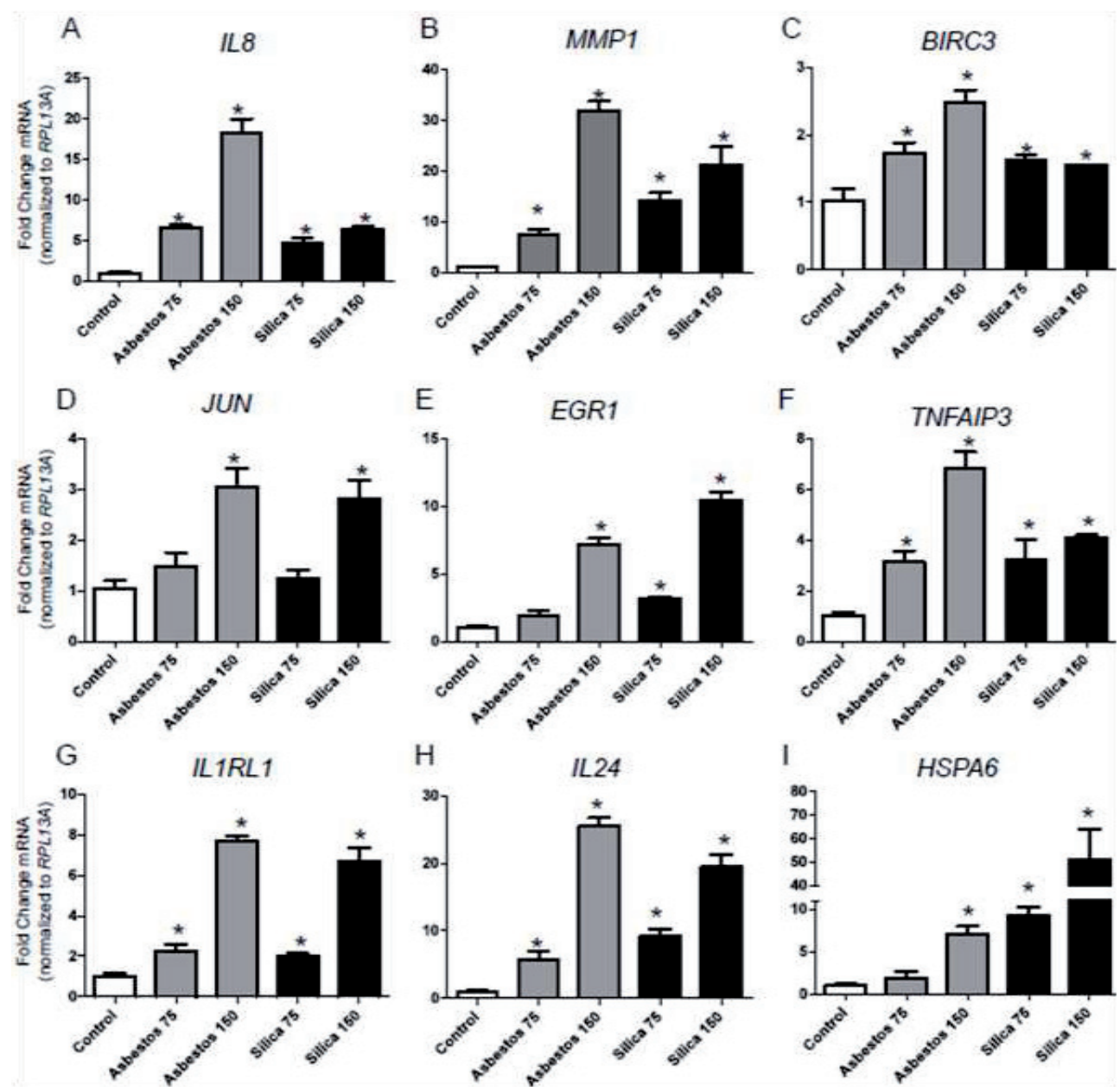

Figure 3: Commonly altered genes by asbestos and silica exposure in BEAS-2B cells. Steady-state mRNA levels were measured by QRT-PCR in BEAS-2B cells exposed to asbestos and silica with two doses (75 and $\left.150 \times 10^{6} \mathrm{\mu m}^{2} / \mathrm{cm}^{2}\right)$ for $24 \mathrm{~h}$. Genes affected by both minerals were (A) IL8 (Interleukin8), (B) MMP1 (Matrix metallopeptidase-1), (C) BIRC3 (Baculoviral IAP-repeat containing 3), (D) JUN (c-jun proto-oncogene), (E) EGR1 (Early growth response-1), (F) TNFAIP3 (TNF $\alpha$-induced protein 3), (G) IL1RL1 (IL-1 receptor-like 1), (H) IL24 (Interleukin-24), (I) HSPA6 (Heat shock protein A6). Gene expression levels were normalized to the housekeeping gene RPL13A (Ribosomal protein $L 13 A$ ) values represent the mean $\pm S E M(*) p<0.05 \mathrm{vs}$. unexposed controls, $N=3$, graphs are representative of 2-3 repeat experiments. 

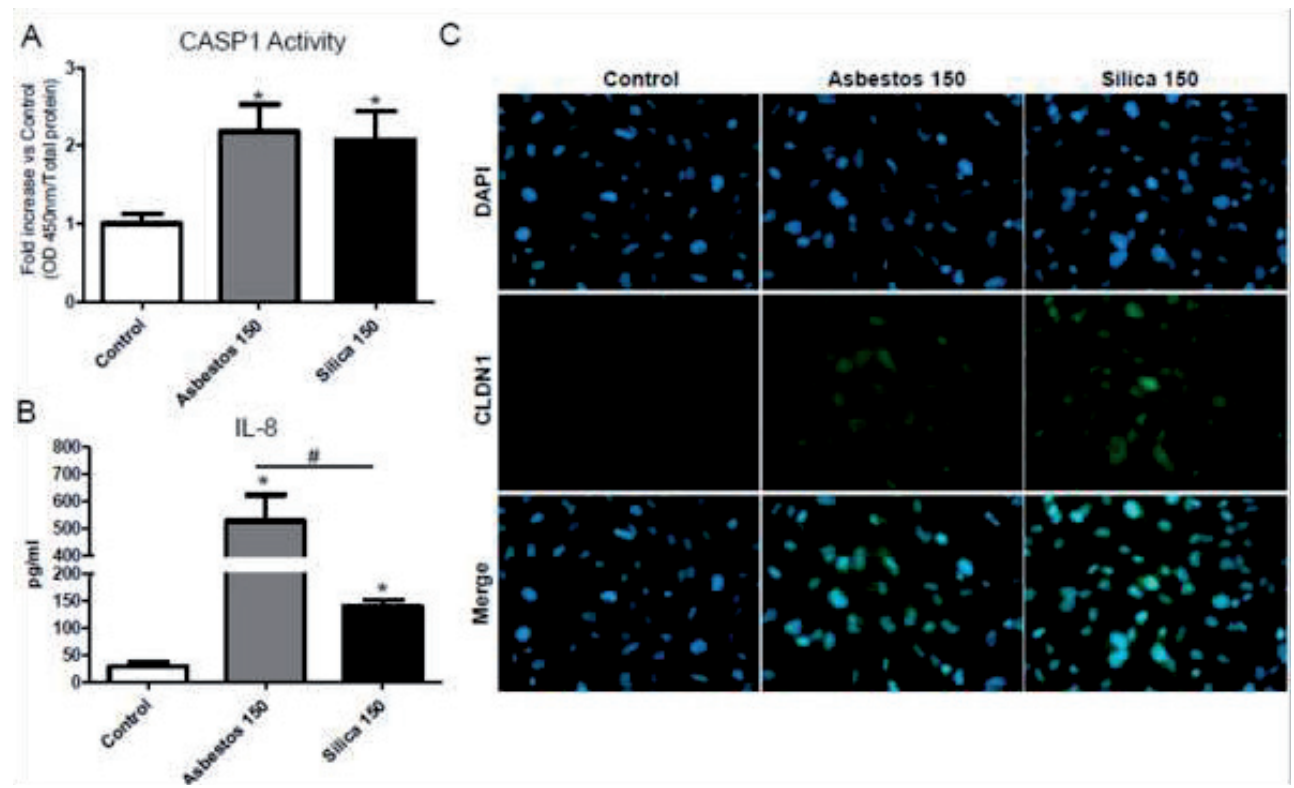

Figure 4: Commonly altered protein by asbestos and silica exposure in BEAS-2B cells. Confirmation of microarray findings at protein level in BEAS-2B cells after $24 \mathrm{~h}$ exposure to asbestos and silica $\left(150 \times 10^{6} \mathrm{\mu m}^{2} / \mathrm{cm}^{2}\right)$. (A) Caspase-1 enzymatic activity levels relative to unexposed controls. (B) Secreted levels of $I L-8$ protein in conditioned medium of $B E A S-2 B \quad(\mathrm{pg} / \mathrm{ml})$. (C) Immunofluorescence images of BEAS-2B (left to right), unexposed controls, asbestos, silica, and nuclear stain DAPI (top), CLDN1 in green (middle), and merged DAPI and CLDN1 (bottom). Negative controls (no primary antibody) were also included and all were negative for staining. Images were taken with $40 X$ objective lens.

\section{Asbestos specific responses}

Genes selectively altered in expression after exposure to crocidolite asbestos $\left(15 \times 10^{6}\right.$ $\mu \mathrm{m}^{2} / \mathrm{cm}^{2}$ ), were grouped in a unique set of pathways which are listed in Table 3 and represented in Figure 5. This collection of experimentally confirmed relationships is associated with biological functions related to tissue damage (ILIRN) and iron homeostasis $(T F R C)$ as well as small molecule biochemistry and cellular detoxification mechanisms (S100A12, CYP2C18 and SULT family members). Further downstream, asbestos specific biological responses were linked to tumor morphology (MGEA5, CRNN, PLCG, and TGMs) and cancer (CEACAM1, SEMA3A) as well as proliferation (FST, NCF1) and cell survival (ABCC10, RBCK1). 
Table 3: Top 10 canonical pathways altered by asbestos specifically in NHBE (24 h). Top 10 canonical pathways significantly enriched by alteration in gene expression by asbestos only as determined by core analysis of asbestos-specific gene changes in IPA.
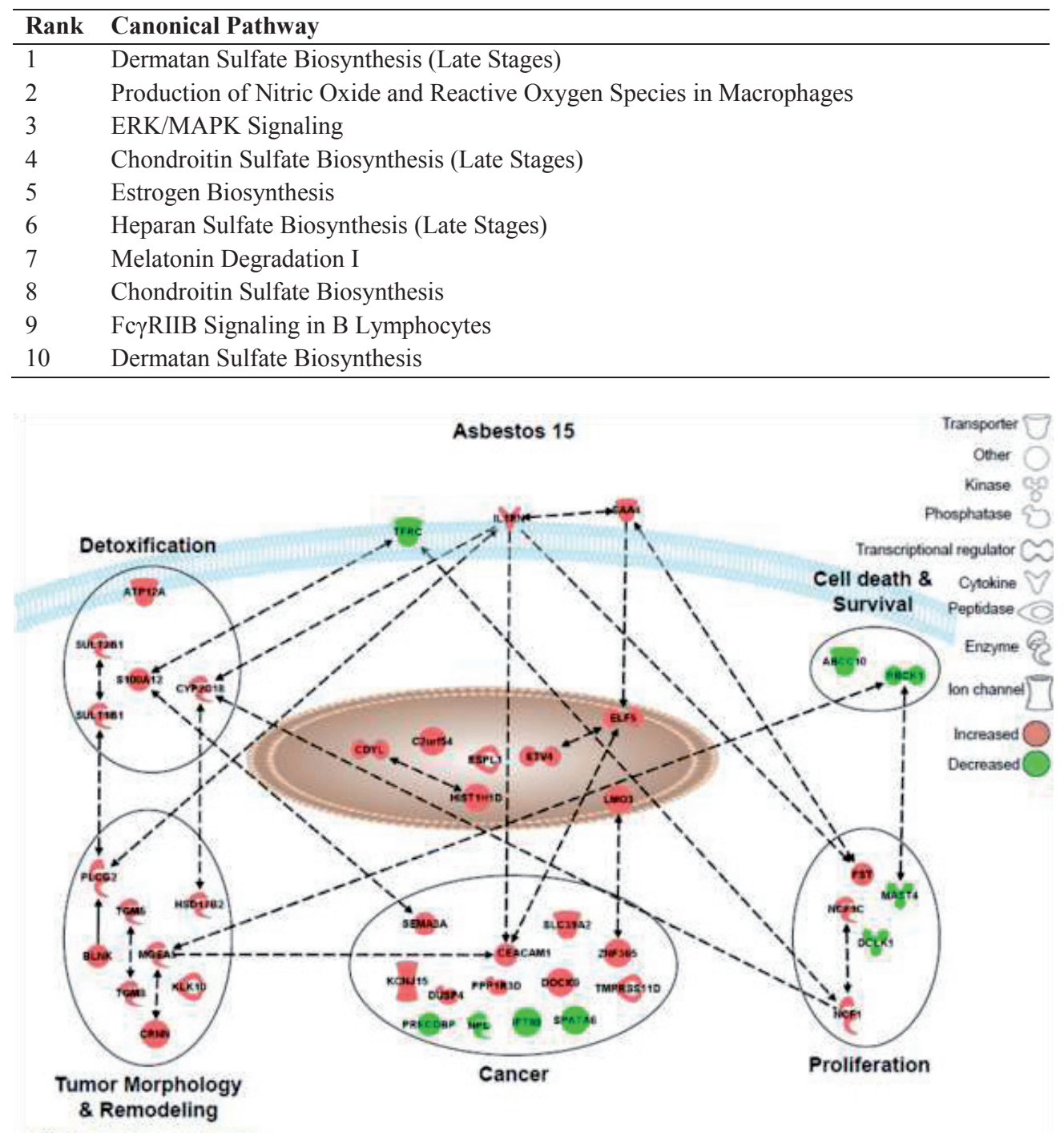

Figure 5: Pathways selectively affected by asbestos in NHBE cells. Graphical representation of the experimentally confirmed relationships between genes distinctively altered in expression by asbestos $\left(15 \times 10^{6} \mathrm{\mu m}^{2} / \mathrm{cm}^{2}\right)$ using IPA software. Red and green colors indicate up-or down-regulation of gene expression levels respectively compared to unexposed control cells [ $\geq 1.5$-fold vs. controls; adj. $p<0.05(F D R<0.05)]$. All gene names, fold-change values, and adj.p-values are listed in Supplemental table 5. 
A selection of these findings was also investigated in the BEAS-2B epithelial cell line by QRT-PCR to confirm microarray-based findings in primary cells. Crocidolite-specific induction of $S E M A 3 A$ expression was confirmed, and interestingly, silica exposure conversely decreased SEMA3A mRNA levels (Figure 6A). In addition to the confirming of crocidolitespecific reduction in TFRC expression, we found that cristobalite silica also had an inverse effect on TFRC mRNA levels in BEAS-2B cells (Figure 6B). Furthermore, exposure to asbestos, but not silica, caused a marked decrease $(50 \%)$ in TFRC protein levels in BEAS-2B cells at $48 \mathrm{~h}$ post-exposure. Silica-induced increases in TFRC mRNA levels (24h), were however not reflected at the protein level at $24 \mathrm{~h}$ and $48 \mathrm{~h}$, which were not significantly affected compared to controls (Figure 6C and data not shown).

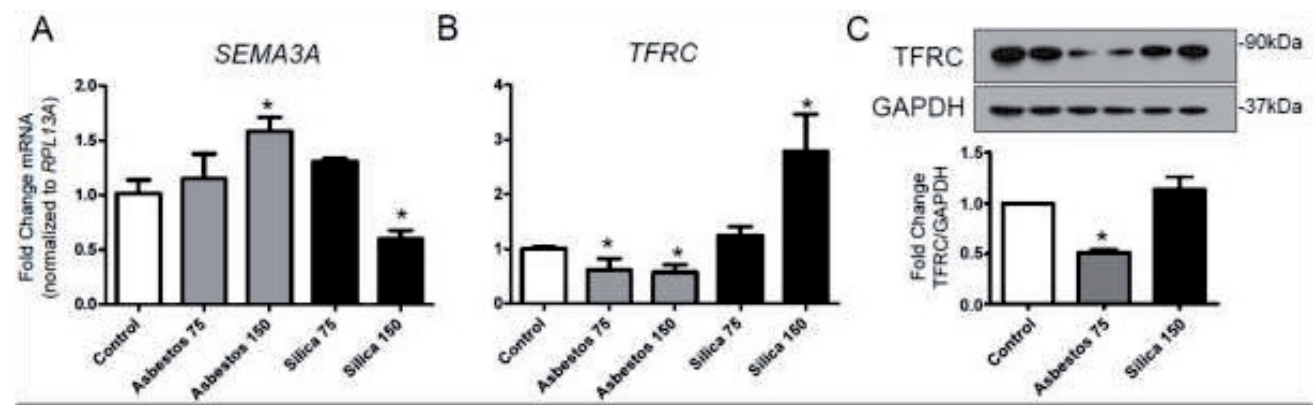

Figure 6: Asbestos-specific genes and protein altered in BEAS-2B cells. Steady-state mRNA levels were measured by QRT-PCR in BEAS-2B cells exposed to asbestos and silica at 2 doses (75 and 150 $X 10^{6} \mathrm{\mu m}^{2} / \mathrm{cm}^{2}$ ) for 24h. Genes significantly altered by asbestos only were (A) SEMA3A (Semaphorin $3 A$ ), (B) TFRC (Transferrin receptor C). Gene expression levels were normalized to the housekeeping gene RPL13A (Ribosomal protein L13A) values represent the mean $\pm S E M\left(^{*}\right) p<0.05$ vs. unexposed controls $(N=3)(C)$ Western blot of TFRC protein levels $(90 \mathrm{kDa})$ and house keeper GAPDH (37 kDa) after exposure to asbestos $\left(75 \times 10^{6} \mathrm{\mu m}^{2} / \mathrm{cm}^{2}\right)$ or silica $\left(150 \times 10^{6} \mathrm{\mu m}^{2} / \mathrm{cm}^{2}\right)$ for $48 \mathrm{~h}$. Densitometry represents relative levels of TFRC normalized to GAPDH $(N=2)$ and graphs are representative of 2-3 repeat experiments.

\section{Silica specific responses}

In NHBE cells, silica exposure at higher concentrations $\left(75 \times 10^{6} \mu \mathrm{m}^{2} / \mathrm{cm}^{2}\right)$ specifically altered expression levels of various cell membrane receptors associated with pathogenassociated molecular pattern (PAMP) recognition ( $L Y 96, T L R 1, C D 14, T R E M 1)$, cell death $(F A S)$, and inflammation (IFNGR1, TNFRSF9, 10B, CXCR4, IL2RG). Additionally, silica exposure affected a number of transcriptional regulators (e.g. IRF, CREB and alternative $\mathrm{NF} \kappa \mathrm{B}$ family members) and a plethora of downstream effector molecules and biological functions including pattern recognition and interferon responses, and additional inflammation, cell survival regulation, and fibrogenesis pathways (Figure 7, Table 4). Figure 8 depicts confirmation of findings in NHBE cells exposed to silica in BEAS-2B cells. Results demonstrate silica-specific up-regulation of the fibrogenic mediator $F G F 2$ (Figure 8A), the inflammatory regulator PTX3 (Figure 8B), the transcriptional regulator CREB5 (Figure 8C), as well as down-regulation of the SFRP1, a modulator of Wingless type (WNT)-signaling 
(Figure 8D), at the highest concentration $\left(150 \times 10^{6} \mu \mathrm{m}^{2} / \mathrm{cm}^{2}\right)$. In addition, we show that silica selectively induced bFGF (FGF2) secretion from bronchial epithelial cells exposed for 24h (Figure 8E).

Table 4: Top 10 canonical pathways altered by silica-specifically in NHBE (24 h). Top 10 canonical pathways significantly enriched by alteration in gene expression by silica only as determined by core analysis of silica-specific gene changes in IPA.
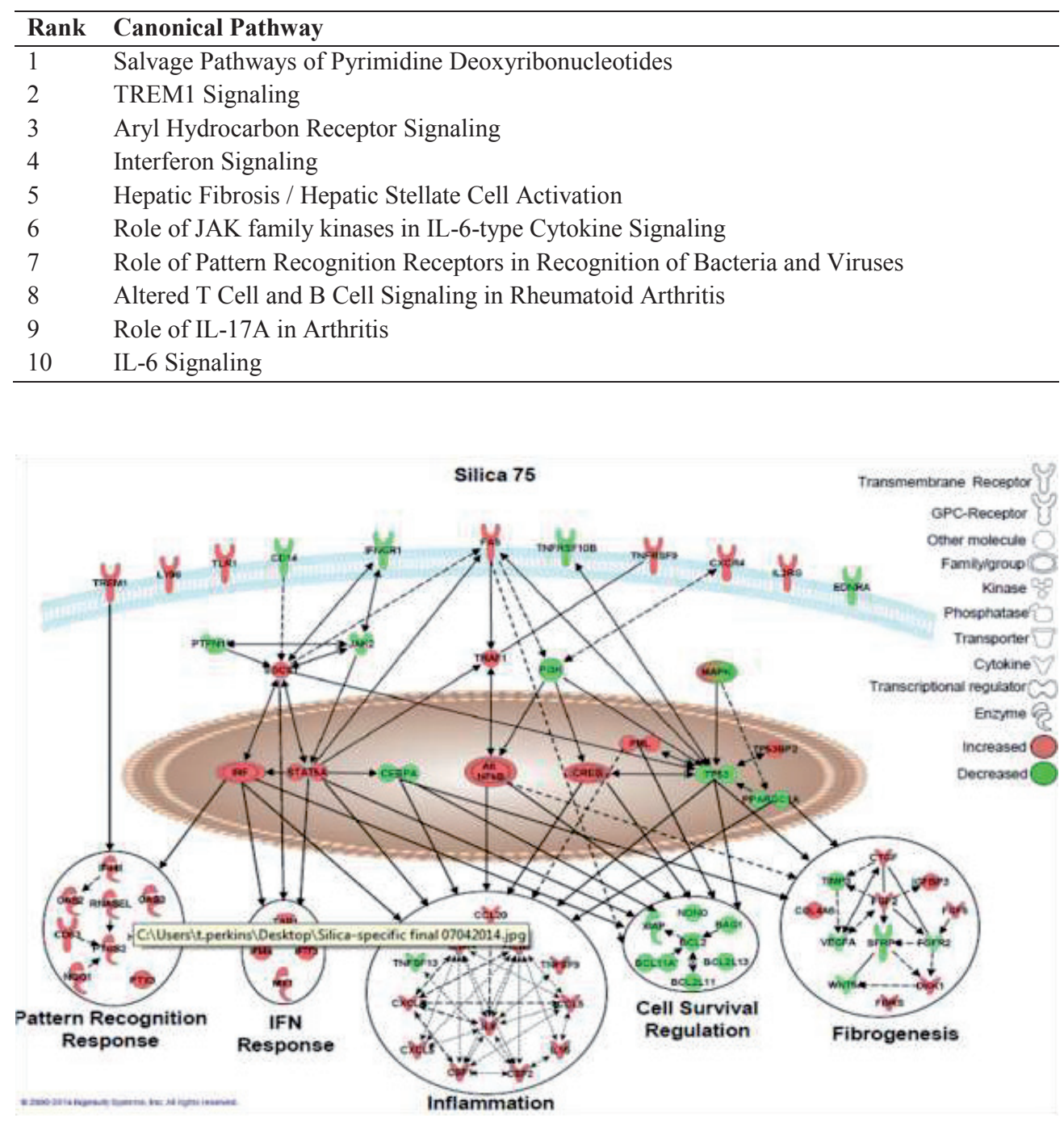

Figure 7: Pathways selectively affected by crystalline silica in NHBE cells. Graphical representation of the experimentally confirmed relationships between genes distinctively altered in expression by silica $\left(75 \times 10^{6} \mathrm{\mu m}^{2} / \mathrm{cm}^{2}\right)$ using IPA software. Red and green colors indicate up- or down-regulation of gene expression levels respectively compared to unexposed control cells $[\geq 1.5$-fold vs. controls; adj. 
p $<0.05(F D R<0.05)]$. All gene names, fold-change values, and adj. p-values are listed in Supplemental table 6.

A

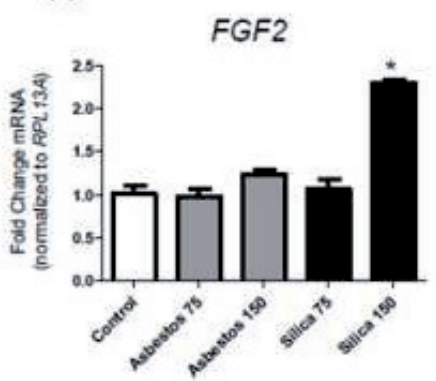

D

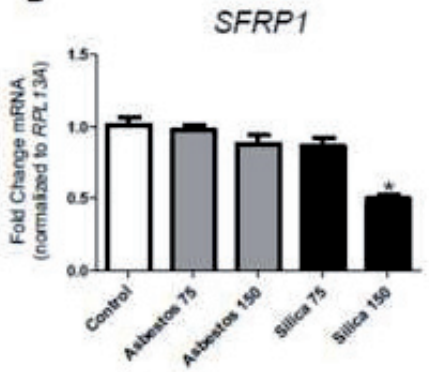

B

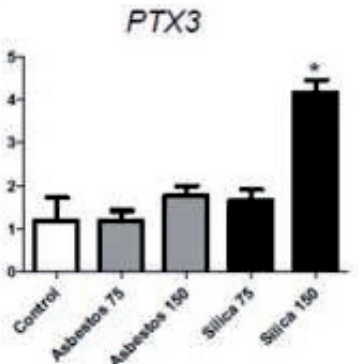

$\mathrm{E}$

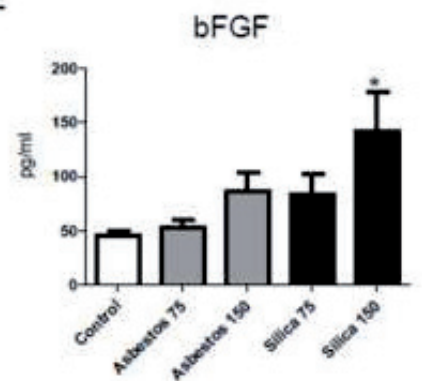

Figure 8: Silica-specific genes and protein altered in BEAS-2B cells. Steady-state mRNA levels were measured by QRT-PCR in BEAS-2B cells exposed to asbestos and silica at two doses (75 and $150 \mathrm{X}$ $10^{6} \mathrm{\mu m}^{2} / \mathrm{cm}^{2}$ ) for $24 \mathrm{~h}$. Genes significantly altered by silica only were (A) FGF2 (fibroblast growth factor 2), (B) PTX3 (Pentraxin 3), (C) CREB5 (cAMP responsive element binding protein 5), and (D) SFRP1 (Secreted frizzled-related protein 1). Gene expression levels were normalized to the housekeeping gene RPL13A (Ribosomal protein L13A). Values represent the mean $\pm S E M(*) p<0.05$ vs. unexposed controls, $N=3$. (E) Secreted levels of bFGF (FGF2) protein in conditioned medium of $B E A S-2 B$ cells exposed $24 \mathrm{~h}(\mathrm{pg} / \mathrm{ml})$. Graphs are representative of 2-3 repeat experiments. 


\section{DISCUSSION}

Our studies demonstrate that robust gene-profiling was able to distinguish different mineral exposures based on global gene expression, as unsupervised average-linkage hierarchical cluster analysis accurately delineated samples based on both mineral exposure, and in the case of silica, the concentration used. Our studies also demonstrate unique features of crocidolite asbestos and cristobalite silica in patterns of toxicity and gene expression alterations. At equal doses $\left(15 \times 10^{6} \mu \mathrm{m}^{2} / \mathrm{cm}^{2}\right)$, silica had minimal effects on gene expression, whereas the number of changes caused by asbestos was dramatically higher. However, at higher doses $\left(75 \times 10^{6}\right.$ $\mu \mathrm{m}^{2} / \mathrm{cm}^{2}$ ), silica induced markedly more gene alterations, mimicking dose-response effects observed in inhalation and intratracheal instillation studies $(23,24)$.

Our data reveal gene changes that likely contribute to the mutual pathogenicity as well as divergent characteristics that may drive the increased carcinogenicity of amphibole asbestos and individual patterns of fibrogenicity. Both minerals altered mRNA levels of various membrane receptors, intracellular signaling molecules, transcriptional regulators and downstream effector molecules some linked to signaling pathways and biological functions that promote the development of mineral exposure-associated disease $(3,25)$. In addition to identifying new genes that may be involved in asbestos and/or silica pathogenicity, our results confirm the highly active role of the epithelium as an initial player in these processes.

\section{Common Responses}

Pathway analysis of genes altered by both minerals revealed that the canonical pathways most highly enriched represented disease mechanisms highly characteristic of the development of both pneumoconioses. These included not only genes well known to be associated with asbestos and silica-disease processes, but also revealed a number of novel genes intrinsic to complex signaling mechanisms that may contribute to disease. Common changes were observed in expression levels of documented membrane receptors associated with proteolysis $(P L A U R)$ and the proinflammatory anaphylatoxin receptor $(C 5 A R)$ as well as its ligand (C5) which has been shown to be activated by both minerals $(26,27)$. Our data also revealed novel genes, associated with propagation of inflammation (IL6R, ILIRLI) and recognition of foreign materials (TLR2). The IL-33 receptor, IL1RL1 (also known as ST2), was up-regulated by both minerals and IL-33 signaling is a driving force in the development of asthma and allergic airways disease $(28,29)$. The lung epithelium is a major source of IL-33 in response to lung injury, propagating signaling to other effector cells (e.g. $\mathrm{T}_{\mathrm{H}} 2$, mast cells, dendritic cells), which may be a feed-forward mechanism. We also have evidence of increased IL-33 secretion from human bronchial epithelial cells by crystalline silica in vitro (unpublished data).

Both AP-1, and NF- $\mathrm{kB}$ have been well established as key transcriptional regulators that drive inflammatory and fibrogenic disease $(3,30,31)$. Moreover, both minerals induced increased levels of EGR1, which is induced by silica in type II alveolar epithelial cells (32) and is associated with epithelial apoptosis, protease expression, and lung fibrosis $(33,34)$. Imbalance of proteolysis and ECM dissolution are key mechanisms in the development of fibrosis (35, 36), by dysregulation of key players such as various MMPs and protease inhibitors. Our study links both asbestos and silica exposure to increased expression of $M M P 1$ in lung epithelial 
cells, which is also increased in human idiopathic pulmonary fibrosis (IPF) (37) and may contribute to further collagen breakdown, activation of cytokines, and promotion of cell migration and proliferation. Increased expression of adherens and junction proteins upon injury to the epithelium is part of the restoration process of the physical barrier. For instance, $C L D N 1$, which is increased in expression after exposure to asbestos and silica here, is also increased in the regenerative epithelium of human IPF and asbestosis lung tissues (21). CLDN1 also has a functional role in regulation of pro-fibrotic processes such as epithelialmesenchymal-transition (EMT) and pro-inflammatory response $(22,38)$. During this response to injury, expression of molecules such as ICAMI and CYR61 promote neutrophil extravasation and migration into the airways. This further perpetuates the potent inflammatory response, which is driven by various mediators including; acute phase proteins, cytokines, and chemokines, which propagates the deregulated cycles of inflammation and injury seen in asbestosis and silicosis.

Activation of the intracellular danger sensing protein complex, the NLRP3 inflammasome, is a crucial step in propagation of the inflammatory response caused by exposure to occupational dusts (e.g. asbestos and silica) $(39,40)$ as well as nanomaterials (e.g. carbon nanotubes) (41). Originally thought to be restricted to myeloid cells, activation has been demonstrated recently in epithelial $(14,42,43)$ and mesothelial cells $(44,45)$. Additionally, certain polymorphisms in the NLRP3 gene may be associated with IL-1 $\beta$ production and severe inflammation which may suggest a genetic predisposition for common inflammatory disorders (46-48).

IL-8, a potent neutrophil chemotactic factor, is a driving force in mineral-induced inflammation, and is shown to be elevated in asbestos (49) and silica-exposed subjects (50). Our data also reveal novel inflammatory molecules increased by both asbestos and silica exposures, including $I L 36 G, I L 36 R N, I L 32$, and SAA2. Interestingly, IL-1 family members,

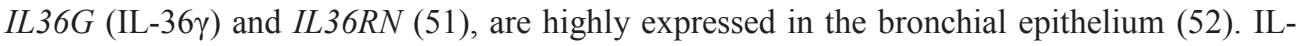
$36 \gamma$ is induced by toll-like receptor (TLR) ligands and $\mathrm{T}_{\mathrm{H}} 17$ cytokines and may promote neutrophilic airway inflammation (53). In conjunction with increased IL-36 $\gamma$ and IL-36Ra, our previous studies show increased expression of their receptors (IL1RL2 and ILIRAP) by crystalline but not amorphous, non-pathogenic silica (14). These seminal data suggest a prominent, and uninvestigated role of IL-36 signaling in mineral exposure-induced inflammation.

Our data in concert suggest a scenario that may be common to pathogenic silicates. Due to massive inflammation and tissue damage caused by exposures to fibrogenic agents, there is a consequent fibrotic response initiated in the epithelium in an attempt to repair damaged areas of the lung. For instance, both minerals increased expression of the profibrotic molecule IL13RA2, which has been shown to promote IL-13 driven induction of TGF $\beta$ in experimental fibrosis (54). Conversely, mineral exposures increased expression of the anti-fibrotic chemokine, CXCL10, which has been shown to alleviate fibrosis in bleomycin-induced experimental lung injury (55). This suggests a balance between anti-fibrotic and pro-fibrotic molecules that may be disrupted in susceptible individuals or at high overload concentrations of minerals. Cell proliferation in response to lung injury, stimulates many biological activities and effector cell types including inflammatory cells and fibroblasts, but may also be compensatory in replenishing epithelial cells after injury and death of surrounding cells. The 
axis balancing cell death and proliferation is often disrupted in chronic disease, as regulation of apoptosis is key to controlling cell turnover of damaged cells. Genes associated with the regulation of apoptosis that were altered by both minerals include the anti-apoptotic molecule, $B I R C 3$, which can be induced by TNF $\alpha$-dependent mechanisms (56), and pro-apoptotic IL24, which is shown to drive apoptosis in various types of cancer cells by inhibition of survival and proliferation signaling and impedes lung cancer cell migration $(57,58)$. In conjunction with exogenous stress, cell death can lead to the excessive release of damaging cellular contents, including oxidants, proteases, and danger molecules, which further propagate cellular stress responses in surrounding tissue. A plethora of heat shock proteins are induced by both minerals, as well as the antioxidant, SOD2. SOD2 has been show to play a pivotal role in mineral-induced disease development (59). Serum levels of SOD2 are elevated in silicotic patients (60) and polymorphisms in this gene are associated with increased asbestosis susceptibility (61).

\section{Asbestos-specific responses}

Many of the genes uniquely affected by crocidolite asbestos have not been described in lung epithelial cells previously, but may have key roles in lung injury and processes leading to carcinogenesis as indicated in Figure 5. Since we, and others have linked asbestos exposures to cellular plasticity and cancer (62-64), it is not surprising that many genes affected by asbestos as opposed to silica are involved in processes related to cancer development. For example, ESPL1 (Separase) acts as an oncogene when overexpressed and plays an important role in the development of aneuploidy and tumorigenesis (65). ELF5 expression appears to be increased significantly in mouse mammary tumors relative to normal tissue (66). Previously, the up-regulation of $S E M A 3 A$ has been linked to a feedback loop modulating growthpromoting signaling in mesothelial cells (67) and influencing apoptosis and proliferation in multiple cell types. SEMA3A, is also essential for structural and functional abnormalities in tumor vascularization (68) and its induction by asbestos in NHBE and BEAS-2B cells, is in line with our recent report on its up-regulation in human mesothelial cells exposed to crocidolite asbestos (69). We were also able to detect elevated CEACAM1 mRNA expression by asbestos. Recently, this gene was linked to the creation of a pro-angiogenic tumor microenvironment that supports tumor vessel maturation (70).

Exposure to foreign toxicants, like asbestos, or other xenobiotic chemicals (e.g. those found in cigarette smoke) activates cellular detoxification mechanisms. For instance, sulfation is an important reaction in detoxification of xenobiotics, and sulfotransferases such as SULT1B1 enzymatically affect the bioactivation of pro-carcinogens to reactive electrophiles (71). In addition to sulfotransferases, asbestos induced increased mRNA levels of CYP2C18, a member of the cytochrome P450 family of detoxification enzymes. Together these data suggest asbestos may activate and potentially disrupt xenobiotic detoxification mechanisms, which is suggested to contribute to asbestos-induced carcinogenic effects (72).

In the group of molecules involved in tumor morphology and remodeling, we observed increased expression levels of various enzymes, including transglutaminases (TGM3, and 5). Rigid amphibole fibers can penetrate and severely disrupt the cell membrane during or after uptake by cells. Upregulation of transglutaminases, which are involved in protein cross- 
linking in molecular scaffolds and wound repair (73), may be part of a repair mechanism in attempt to restore the compromised a cell membrane.

The activin inhibitor FST has been shown to ameliorate inflammation and fibro-proliferative responses in a bleomycin-model of pulmonary fibrosis in rats $(74,75)$. It is possible that the up-regulation of FST, as observed here in the proliferation pathway in response to asbestos, is part of an endogenous defense mechanism of bronchial epithelial cells.

For amphibole asbestos, the surface chemistry, particularly bioavailable iron, is key in driving oxidant production and inflammation $(76,77)$. Iron is mobilized and promotes oxidant production in lung epithelial cells exposed to crocidolite asbestos. Additionally, endogenous iron coats asbestos fibers in the lung, i.e. ferruginous bodies, one of the hallmarks of asbestosis $(78,79)$. Iron sequestration is controlled by a number of molecules, including transferrin and the transferrin receptor (80). Our data indicate significant decreases in mRNA and protein levels of TFRC in human lung epithelial cells exposed to crocidolite asbestos. Crocidolite exposure causes intracellular sequestration of non-transferrin bound iron, potentially via the divalent metal ion transporter-1 (DMT1) (81). Accumulation of such intracellular iron stores likely leads to the down-regulation of TFRC, which in turn, may increase the amount of extracellular iron. Bronchoalveolar lavage (BAL) fluids from asbestosexposed subjects have higher levels of transferrin compared to unexposed subjects (82). Therefore, inhalation of asbestos can lead to severe disruption of normal iron homeostasis in the lungs, which may be a potential marker for exposure and/or disease development.

\section{Silica specific responses}

The presence of massive inflammation is a driving force in the development and progression of silicosis. Our data show a robust inflammatory response to high dose silica exposure with specifically increased expression of many potent inflammatory mediators. Silica induced for instance $I L 1 A, I L 1 B$, colony stimulating factors, CSF1, CSF2, as well as (C-X-C) and (C-C) motif chemokines. Interestingly, silica induced increased expression of TNFSF9 (CD137L/41BB) as well as its receptor TNFRSF9. TNFSF9 signaling is a key factor in the development of immune responses in T-cells, NK cells, macrophages and dendritic cells, and is also expressed in non-immune cells. Crosstalk between epithelial cells and inflammatory cells via TNFSF9-signaling drives neutrophil recruitment (83) which contribute to silica-induced inflammation. In keeping with these findings, there was also an evident interferon response. Type I interferon responses have been shown to play a role in silica-induced chronic inflammation (84), however, the mechanism by which they contribute to silica-induced inflammation is not well understood. In line with progressive inflammation, and interferon response, silica exposure was associated with pattern-recognition signaling pathways, with altered expression of membrane and intracellular pattern recognition-associated receptors (LY96, TLR1, CD14, and IFIH1/MDA-5). In addition, silica caused increases in antiviralresponse proteins $(O A S)$, and antigen-presentation molecules (CD83, HLADQB1). Silica also caused increased expression of the multifunctional pattern recognition molecule, PTX3 (85) which has been implicated in epithelial cell survival in co-culture models of silica-exposure (86). 
In response to silica particles, expression levels of a number of cell membrane receptors that mediate PAMP recognition, cell death, and proinflammatory response were specifically altered. Silica exposure is associated with development of autoimmunity, where increased serum levels of (soluble) sIL2R, are a marker of early immunological dysfunction in silicaexposed subjects (87). This correlates with our data, which reveals increased levels of IL2RG (i.e. the common gamma chain) mRNA. Furthermore, TNF $\alpha$ and the TNFR signaling pathway have been shown to be crucial mediators in the development of silica-induced fibrosis (88) and soluble TNFR1 and TNFR2 are increased in subjects exposed to silica (89).with In conjunction with the up-regulated expression of a number of the TNF-receptor superfamily members, our data reveal up-regulation of the adaptor protein TRAF1, which is

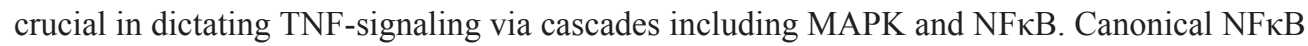
signaling is well-known to drive silica-induced inflammation and fibrosis $(90,91)$. However, our data are the first to reveal modulation of non-canonical (alternative) NFKB signaling, by up-regulation of NFKB2 (p52/p100) and RELB. Alternative NFKB signaling and autoregulation are shown to modulate lung inflammation $(92,93)$ and cross-talk between canonical and non-canonical $\mathrm{NF} \kappa \mathrm{B}$ regulates inflammatory responses in epithelial cells as well as airway inflammation and fibrosis in experimental models of allergic airways disease $(94,95)$.

Due to initial injury and the inability to clear silica particles, followed by and cycles of inflammation that precede and continue during the development of silicosis. Exposure to silica promotes the production of various fibrogenic mediators, proteases, and proliferation and recruitment of fibroblasts in attempt to repair the damaged lung. Our data reveal silica specific alteration of various fibrogenic mediators (e.g. VEGF, CTGF, FBRS, FGF2) and downstream signaling molecules associated with the fibrogenesis pathway. In particular, silica specifically induced mRNA levels and secretion of $F G F 2 / b F G F$. This potent fibrogenic factor (and others associated), which is induced by silica in lung epithelial cells $(9,86)$ and potentially linked indirectly to fibroblast proliferation (14) may have a crucial impact on the intense fibrogenicity of silica. Furthermore, although WNT-signaling is linked with aberrant proliferation and the development of fibrosis $(96,97)$, it has not been associated with silica or silicosis yet. Interestingly we observed down-regulation of the WNT-signaling molecules SFRP1, WNT5A and up-regulation of DKK1 in response to silica. These findings suggest potential alteration of WNT-signaling.

In summary, our study exemplifies how gene expression studies can predict acute responses of respiratory epithelial cells, the first cell type to encounter asbestos fibers or silica particles after inhalation, and link these events to biofunctional pathways leading to lung disease. Many of these alterations can be linked to biomarkers found in clinical samples of exposed subjects. Overall, our data reveal early alterations in both common gene expression that can be linked to inflammation and fibrosis by both pathogenic minerals and unique changes by silica promoting intense fibrogenicity and by asbestos that may be intrinsic to the development of lung cancers. Our analyses also highlight many novel molecules that can be pursued in functional studies, thus permitting their evaluation as new targets in detection, prevention and therapy of fibroproliferative diseases and cancers. 


\section{MATERIALS AND METHODS}

\section{Cell culture}

For primary cells, an isolate of normal primary human bronchial epithelial cells (NHBE) was purchased from Lonza, Clonetics ${ }^{\circledR}$. NHBE cells were cultured and maintained in BEGM ${ }^{\circledR}$ with Reagentpack ${ }^{\mathrm{TM}}$ subculture reagents as previously described (9). For experiments with BEAS-2B cells, cells were cultured as recommended by ATCC as described previously (9).

\section{Fiber/particle exposures}

Respirable preparations of crocidolite asbestos fibers (NIEHS reference sample) and cristobalite silica particles (C\&E Mineral Corp, King of Prussia, PA) were sterilized and diluted to a concentration of $1.0 \mathrm{mg} / \mathrm{ml}$ prior to cell exposures at equal surface areas as described previously (9). Control samples were exposed to an equal volume of Hank's buffered saline solution (HBSS) alone (no-particle/fiber control) The fiber dimension data for this asbestos sample was previously reported (98), and particle characterization of cristobalite silica has previously been published $(9,59)$.

\section{RNA isolation}

After exposure of cells, medium was aspirated and total RNA was isolated using an RNeasy ${ }^{\circledR}$ Plus Mini Kit according to the manufacturers' protocol (Qiagen, Valencia, CA, USA) for NHBE cells. For BEAS-2B cell experiments, total RNA was isolated using the High Pure RNA Isolation Kit according to the manufacturers' protocol (Roche Applied Sciences, Indianapolis, IN, USA)

\section{Quantitative real time polymerase chain reaction (QRT-PCR)}

For primary NHBE experiments, total RNA $(1 \mu \mathrm{g})$ was reverse-transcribed with random primers using the AMV Reverse Transcriptase kit (Promega,Madison, WI) according to the recommendations of the manufacturer, as described previously (15). All Taqman ${ }^{\circledR}$ qRT-PCR was performed using Assays-On-Demand ${ }^{\mathrm{TM}}$ primer and probe sets (IL8, MMPI, BIRC3) used from Applied Biosystems (Foster City, CA) and performed as described previously (15). For all BEAS-2B experiments QRT-PCR was performed as previously described (99). All forward and reverse primer sequences are described in supplemental material.

\section{Affymetrix microarray gene expression profiling}

Microarray analysis was performed using Affymetrix GeneChip ${ }^{\circledR}$ Human Genome U133A 2.0 arrays (Affymetrix, Santa Clara, CA). All procedures were performed by the Microarray Analysis Core Facility of the Vermont Genetics Network (VGN) using a standard Affymetrix protocol as described previously $(100,101)$. GeneChip ${ }^{\circledR}$ Human Genome U133A 2.0 arrays (Affymetrix, Santa Clara, CA) targeting 18,400 genes were scanned twice (Hewlett-Packard GeneArray Scanner), the images overlaid, and the average intensities of each probe cell compiled.

Microarray data were analyzed with Bioconductor tools in $\mathrm{R}$ with Bioconductor software (http://www.r-project.org/) (102), probe level analysis was performed on the Affymetrix raw data (.cel files) with the robust multichip average (RMA) method to obtain a $\log 2$ 
expression value for each gene probe set (103, 104). Unsupervised average-linkage hierarchical clustering, using Euclidian distance as metric, was performed to the RMA normalized data to visualize gene (probe set)/sample relationships of the top 50 most variable probe-set (103).

\section{GeneSifter analysis}

Following data normalization using the RMA algorithm, the GeneSifter software (VizX Labs, Seattle, WA) was used for supervised hierarchical cluster analysis to identify probe sets/genes from $\log _{2}$-transformed data that significantly changed expression between the different groups (unexposed control, silica 15, asbestos 15 and silica 75; $\mathrm{N}=3$ per sample group) by pair-wise comparison analysis ( $t$-test), and for the entire experimental group by multiple comparison analysis procedures (ANOVA). The raw p-values were corrected for multiple testing using the Benjamini and Hochberg false discovery rate method (FDR $<95 \%$ ) or "adjusted p-value" (105). A cut-off limit of 1.5-fold change vs. controls (adj. $\mathrm{p}<0.05$ ) was used for analysis.

\section{Ingenuity pathway analysis}

Comparative analysis of pair-wise data-sets (Asbestos 15 vs. controls; and Silica 75 vs. controls) revealed three groups of observations: 1) probe-sets significantly altered by both (asbestos 15 vs. controls) and (silica 75 vs. controls), 2) probe-sets significantly altered by (asbestos 15 vs. controls) only, and 3) probe-sets significantly altered by (silica 75 vs. controls) only. These data sets were uploaded with gene ID (affymetrix), fold-change value, and adj. p-value in the Ingenuity Pathway Analysis program (IPA, Ingenuity Systems ${ }^{\circledR}$, www.ingenuity.com) for core analysis. Core analysis of each data-set was performed (confidence level: experimentally observed). Genes associated with the top 10 canonical pathways significantly enriched by each comparison, in addition to a selection of genes with evident relevance to the canonical pathways and biological function were used to construct schematic representations. The significance of the association between the input data set and the canonical pathways was determined based on two parameters: (1) A ratio of the number of genes from the data set that overlay to the pathway divided by the total number of genes that map to the canonical pathway and (2) a P-value calculated determining the probability that the association between the genes in the data set and the canonical pathway is not due to chance alone. Using the "connect" tool, experimentally observed, direct, and indirect connections were made for based on the Ingenuity Knowledge Base (Ingenuity Systems Inc., Redwood City, CA). For clarity purposes, not all connections are shown, but only those pertinent to the biological scheme.

\section{Immunofluorescence}

For immunofluorescent staining of BEAS2B cells, sterile glass coverslips (VWR 13mm, Thickness No.1) were placed in 24-well culture plates and coated with coating medium overnight at $37^{\circ} \mathrm{C}$. Cells were seeded, maintained and exposed to minerals as previously described (9). Following exposure culture medium was aspirated, and cells washed 3 times with $1 \mathrm{X}$ PBS. Cells were then fixed in $4 \%$ phosphate buffered formaldehyde solution $\mathrm{pH} 7.0$ (Klinipath 4078-9001) for 20 minutes at RT. Cells were washed 3 times with PBS and placed 
in blocking/permeabilizations buffer (1.0\% BSA, $0.1 \%$ Triton X-100 in PBS) for $1 \mathrm{~h}$ at RT. Cells were incubated in primary antibody $\left(1: 100\right.$ dilution) overnight at $4^{\circ} \mathrm{C}$. Cells were washed 3 times with PBS, incubated with an Alexa Fluor ${ }^{\circledR}$ conjugated secondary antibody (1:1000 in 1\%BSA-PBS) for 1 hour at RT (in dark). Cells were washed 3 times with PBS and incubated with DAPI solution $(0.5 \mu \mathrm{g} / \mathrm{ml})$ for 5 minutes at RT (in dark). Cells were washed 2 times with PBS and once with distilled water. Coverslips were then mounted on glass slides (Knittel Glass, Germany) using fluorescent mounting medium (DAKO). Cells were imaged using Zeiss ZEN (blue) 2012 software. Images were exported in .TIFF format

\section{Western blot}

Cells were washed twice with cold HBSS, and lysed with 1X RIPA buffer [(RIPA buffer 10X Cell Signaling) with 1\% Protease inhibitor cocktail (Sigma), 1\% Sodium orthovanadate, $1 \%$ PMSF, and $1 \mathrm{mM} \mathrm{DTT].} \mathrm{Total} \mathrm{protein} \mathrm{was} \mathrm{determined} \mathrm{using} \mathrm{the} \mathrm{Bio-Rad} \mathrm{DC-protein}$ determination kit (Bio-Rad, Hercules CA, USA). $20 \mu \mathrm{g}$ of protein was loaded onto pre-cast $12 \%$ (bis-tris) polyacrylamide gels (Bio-Rad). Gels were run, and transferred to nitrocellulose membranes. After blocking in 5\% milk, membranes were incubated with primary antibodies (Anti-TFRC 1:5000, Anti-GAPDH 1:20,000) overnight at $4^{\circ} \mathrm{C}$. Membranes were washed 3 times $15 \mathrm{~min}$. in TBS-Tween $(0.1 \%)$, and then incubated in HRP-conjugated secondary antibody (1:5000 in 5\% milk) for $1 \mathrm{~h}$ at RT. Membranes were washed 3 times in TBS-Tween $(0.1 \%)$ and once in TBS, and then incubated in ECL substrate and developed. Densitometry was performed using QuantityOne software (Bio-Rad).

\section{Caspase-1 activity assay}

Caspase-1 activity was measured using a caspase-1 activity kit (R\&D) following the manufacturer's protocol.

\section{ELISA}

To determine levels of bFGF and IL-8 levels in cell culture medium, culture medium was centrifuged for $10 \mathrm{~min}$. at $14,000 \mathrm{rpm}\left(4^{\circ} \mathrm{C}\right)$ to remove all cellular debris and minerals, and supernatant removed. Levels of bFGF and IL-8 in the supernatant were determined using a Human FGF-basic ELISA MAX Deluxe kit (Biolegend) and a Human IL-8 ELISA kit (Sanquin) respectively following the manufacturers' protocols.

\section{Antibodies}

Primary antibodies: Rabbit polyclonal Anti-Transferrin Receptor C (Sino Biological corp., \#11020-RP02), Rabbit polyclonal anti-GAPDH (Cell Signaling), rabbit polyclonal antiClaudin-1 (Cell Signaling \#4933). Secondary Antibodies: Alexa Fluor ${ }^{\circledR} 488$ conjugated antirabbit IgG (Life Technologies), and Peroxidase-conjugated anti-rabbit IgG (Vector Laboratories).

\section{Statistical analyses}

All statistical analyses for microarray and pathway analysis data are described above in their respective sections. For cell viability and QRT-PCR, data were analyzed by one-way analysis 
of variance (ANOVA) using the Student Neuman-Keul's test to adjust for multiple pair-wise comparisons between treatment groups, or the Student's $t$ test where appropriate. Data are expressed as mean values \pm SEM. Differences with p-value $<0.05$ were considered statistically significant. Data presented are representative of multiple independent repeat experiments.

\section{REFERENCES}

1 Chong, S., Lee, K.S., Chung, M.J., Han, J., Kwon, O.J. and Kim, T.S. (2006) Pneumoconiosis: comparison of imaging and pathologic findings. Radiographics, 26, 59-77.

2 Guthrie, G.D., Jr. and Heaney, P.J. (1995) Mineralogical characteristics of silica polymorphs in relation to their biological activities. Scand J Work Environ Health, 21 Suppl 2, 5-8.

3 Mossman, B.T. and Churg, A. (1998) Mechanisms in the pathogenesis of asbestosis and silicosis. Am J Respir Crit Care Med, 157, 1666-1680.

4 Borm, P.J., Tran, L. and Donaldson, K. (2011) The carcinogenic action of crystalline silica: a review of the evidence supporting secondary inflammation-driven genotoxicity as a principal mechanism. Crit Rev Toxicol, 41, 756-770.

5 Gamble, J.F. (2011) Crystalline silica and lung cancer: a critical review of the occupational epidemiology literature of exposure-response studies testing this hypothesis. Crit Rev Toxicol, 41, 404-465.

6 Guha, N., Straif, K. and Benbrahim-Tallaa, L. (2011) The IARC Monographs on the carcinogenicity of crystalline silica. Med Lav, 102, 310-320.

7 Leung, C.C., Yu, I.T. and Chen, W. (2012) Silicosis. Lancet, 379, 2008-2018.

8 Castranova, V. and Vallyathan, V. (2000) Silicosis and coal workers' pneumoconiosis. Environ Health Perspect, 108 Suppl 4, 675-684.

9 Perkins, T.N., Shukla, A., Peeters, P.M., Steinbacher, J.L., Landry, C.C., Lathrop, S.A., Steele, C., Reynaert, N.L., Wouters, E.F. and Mossman, B.T. (2012) Differences in gene expression and cytokine production by crystalline vs. amorphous silica in human lung epithelial cells. Part Fibre Toxicol, 9,6 .

10 Shukla, A., MacPherson, M.B., Hillegass, J., Ramos-Nino, M.E., Alexeeva, V., Vacek, P.M., Bond, J.P., Pass, H.I., Steele, C. and Mossman, B.T. (2009) Alterations in gene expression in human mesothelial cells correlate with mineral pathogenicity. Am J Respir Cell Mol Biol, 41, 114-123.

11 Scapoli, L., Ramos-Nino, M.E., Martinelli, M. and Mossman, B.T. (2004) Src-dependent ERK5 and Src/EGFR-dependent ERK1/2 activation is required for cell proliferation by asbestos. Oncogene, 23, 805-813. 
12 Shukla, A., Barrett, T.F., Nakayama, K.I., Nakayama, K., Mossman, B.T. and Lounsbury, K.M. (2006) Transcriptional up-regulation of MMP12 and MMP13 by asbestos occurs via a PKCdeltadependent pathway in murine lung. Faseb J, 20, 997-999.

13 Fubini, B. and Hubbard, A. (2003) Reactive oxygen species (ROS) and reactive nitrogen species (RNS) generation by silica in inflammation and fibrosis. Free Radic Biol Med, 34, 1507-1516.

14 Peeters, P.M., Perkins, T.N., Wouters, E.F., Mossman, B.T. and Reynaert, N.L. (2013) Silica induces NLRP3 inflammasome activation in human lung epithelial cells. Part Fibre Toxicol, 10, 3.

15 Hillegass, J.M., Shukla, A., MacPherson, M.B., Lathrop, S.A., Alexeeva, V., Perkins, T.N., van der Vliet, A., Vacek, P.M., Gunter, M.E. and Mossman, B.T. (2010) Mechanisms of oxidative stress and alterations in gene expression by Libby six-mix in human mesothelial cells. Part Fibre Toxicol, 7, 26.

16 Huang, Y.C., Karoly, E.D., Dailey, L.A., Schmitt, M.T., Silbajoris, R., Graff, D.W. and Devlin, R.B. (2011) Comparison of gene expression profiles induced by coarse, fine, and ultrafine particulate matter. J Toxicol Environ Health A, 74, 296-312.

17 Hillegass, J.M., Shukla, A., MacPherson, M.B., Bond, J.P., Steele, C. and Mossman, B.T. (2010) Utilization of gene profiling and proteomics to determine mineral pathogenicity in a human mesothelial cell line (LP9/TERT-1). J Toxicol Environ Health A, 73, 423-436.

18 Ding, M., Shi, X., Dong, Z., Chen, F., Lu, Y., Castranova, V. and Vallyathan, V. (1999) Freshly fractured crystalline silica induces activator protein-1 activation through ERKs and p38 MAPK. $J$ Biol Chem, 274, 30611-30616.

19 Heintz, N.H., Janssen, Y.M. and Mossman, B.T. (1993) Persistent induction of c-fos and c-jun expression by asbestos. Proc Natl Acad Sci U S A, 90, 3299-3303.

20 Heintz, N.H., Janssen-Heininger, Y.M. and Mossman, B.T. (2010) Asbestos, lung cancers, and mesotheliomas: from molecular approaches to targeting tumor survival pathways. Am J Respir Cell Mol Biol, 42, 133-139.

21 Lappi-Blanco, E., Lehtonen, S.T., Sormunen, R., Merikallio, H.M., Soini, Y. and Kaarteenaho, R.L. (2013) Divergence of tight and adherens junction factors in alveolar epithelium in pulmonary fibrosis. Hum Pathol, 44, 895-907.

22 Shiozaki, A., Bai, X.H., Shen-Tu, G., Moodley, S., Takeshita, H., Fung, S.Y., Wang, Y., Keshavjee, S. and Liu, M. (2012) Claudin 1 mediates TNFalpha-induced gene expression and cell migration in human lung carcinoma cells. PLoS One, 7, e38049.

23 Mossman, B.T. and Glenn, R.E. (2013) Bioreactivity of the crystalline silica polymorphs, quartz and cristobalite, and implications for occupational exposure limits (OELs). Crit Rev Toxicol, 43, $632-660$.

24 Mossman, B.T., Lippmann, M., Hesterberg, T.W., Kelsey, K.T., Barchowsky, A. and Bonner, J.C. (2011) Pulmonary endpoints (lung carcinomas and asbestosis) following inhalation exposure to asbestos. J Toxicol Environ Health B Crit Rev, 14, 76-121.

25 Vallyathan, V., Shi, X. and Castranova, V. (1998) Reactive oxygen species: their relation to pneumoconiosis and carcinogenesis. Environ Health Perspect, 106 Suppl 5, 1151-1155. 
26 Governa, M., Amati, M., Valentino, M., Visona, I., Fubini, B., Botta, G.C., Volpe, A.R. and Carmignani, M. (2000) In vitro cleavage by asbestos fibers of the fifth component of human complement through free-radical generation and kallikrein activation. $J$ Toxicol Environ Health $A, \mathbf{5 9}, 539-552$.

27 Governa, M., Fenoglio, I., Amati, M., Valentino, M., Bolognini, L., Coloccini, S., Volpe, A.R., Carmignani, M. and Fubini, B. (2002) Cleavage of the fifth component of human complement and release of a split product with $\mathrm{C} 5$ a-like activity by crystalline silica through free radical generation and kallikrein activation. Toxicol Appl Pharmacol, 179, 129-136.

28 Kamekura, R., Kojima, T., Takano, K., Go, M., Sawada, N. and Himi, T. (2012) The role of IL33 and its receptor ST2 in human nasal epithelium with allergic rhinitis. Clin Exp Allergy, 42, 218-228.

29 Yagami, A., Orihara, K., Morita, H., Futamura, K., Hashimoto, N., Matsumoto, K., Saito, H. and Matsuda, A. (2010) IL-33 mediates inflammatory responses in human lung tissue cells. $J$ Immunol, 185, 5743-5750.

30 Castranova, V. (2004) Signaling pathways controlling the production of inflammatory mediators in response to crystalline silica exposure: role of reactive oxygen/nitrogen species. Free Radic Biol Med, 37, 916-925.

31 Rimal, B., Greenberg, A.K. and Rom, W.N. (2005) Basic pathogenetic mechanisms in silicosis: current understanding. Curr Opin Pulm Med, 11, 169-173.

32 Chu, L., Wang, T., Hu, Y., Gu, Y., Su, Z. and Jiang, H. (2013) Activation of Egr-1 in human lung epithelial cells exposed to silica through MAPKs signaling pathways. PLoS One, 8, e68943.

33 Lee, C.G., Cho, S.J., Kang, M.J., Chapoval, S.P., Lee, P.J., Noble, P.W., Yehualaeshet, T., Lu, B., Flavell, R.A., Milbrandt, J. et al. (2004) Early growth response gene 1-mediated apoptosis is essential for transforming growth factor beta1-induced pulmonary fibrosis. J Exp Med, 200, 377389.

34 Xiang, F., Bai, M., Jin, Y., Ma, W. and Xin, J. (2007) Egr-1 mediates Si0(2)-driven transcription of membrane type I matrix metalloproteinase in macrophages. J Huazhong Univ Sci Technolog Med Sci, 27, 13-16.

35 Scabilloni, J.F., Wang, L., Antonini, J.M., Roberts, J.R., Castranova, V. and Mercer, R.R. (2005) Matrix metalloproteinase induction in fibrosis and fibrotic nodule formation due to silica inhalation. Am J Physiol Lung Cell Mol Physiol, 288, L709-717.

36 Tan, R.J., Fattman, C.L., Niehouse, L.M., Tobolewski, J.M., Hanford, L.E., Li, Q., Monzon, F.A., Parks, W.C. and Oury, T.D. (2006) Matrix metalloproteinases promote inflammation and fibrosis in asbestos-induced lung injury in mice. Am J Respir Cell Mol Biol, 35, 289-297.

37 Herrera, I., Cisneros, J., Maldonado, M., Ramirez, R., Ortiz-Quintero, B., Anso, E., Chandel, N.S., Selman, M. and Pardo, A. (2013) Matrix metalloproteinase (MMP)-1 induces lung alveolar epithelial cell migration and proliferation, protects from apoptosis, and represses mitochondrial oxygen consumption. J Biol Chem, 288, 25964-25975.

38 Suh, Y., Yoon, C.H., Kim, R.K., Lim, E.J., Oh, Y.S., Hwang, S.G., An, S., Yoon, G., Gye, M.C., Yi, J.M. et al. (2013) Claudin-1 induces epithelial-mesenchymal transition through activation of the c-Abl-ERK signaling pathway in human liver cells. Oncogene, 32, 4873-4882. 
39 Cassel, S.L., Eisenbarth, S.C., Iyer, S.S., Sadler, J.J., Colegio, O.R., Tephly, L.A., Carter, A.B., Rothman, P.B., Flavell, R.A. and Sutterwala, F.S. (2008) The Nalp3 inflammasome is essential for the development of silicosis. Proc Natl Acad Sci U S A, 105, 9035-9040.

40 Dostert, C., Petrilli, V., Van Bruggen, R., Steele, C., Mossman, B.T. and Tschopp, J. (2008) Innate immune activation through Nalp3 inflammasome sensing of asbestos and silica. Science, 320, 674-677.

41 Yazdi, A.S., Guarda, G., Riteau, N., Drexler, S.K., Tardivel, A., Couillin, I. and Tschopp, J. (2010) Nanoparticles activate the NLR pyrin domain containing 3 (Nlrp3) inflammasome and cause pulmonary inflammation through release of IL-1alpha and IL-1beta. Proc Natl Acad Sci U $S$ A, 107, 19449-19454.

42 Hussain, S., Sangtian, S., Anderson, S.M., Snyder, R.J., Marshburn, J.D., Rice, A.B., Bonner, J.C. and Garantziotis, S. (2014) Inflammasome activation in airway epithelial cells after multi-walled carbon nanotube exposure mediates a profibrotic response in lung fibroblasts. Part Fibre Toxicol, 11, 28 .

43 Hirota, J.A., Hirota, S.A., Warner, S.M., Stefanowicz, D., Shaheen, F., Beck, P.L., Macdonald, J.A., Hackett, T.L., Sin, D.D., Van Eeden, S. et al. (2012) The airway epithelium nucleotidebinding domain and leucine-rich repeat protein 3 inflammasome is activated by urban particulate matter. J Allergy Clin Immunol, 129, 1116-1125 e1116.

44 Hillegass, J.M., Miller, J.M., MacPherson, M.B., Westbom, C.M., Sayan, M., Thompson, J.K., Macura, S.L., Perkins, T.N., Beuschel, S.L., Alexeeva, V. et al. (2013) Asbestos and erionite prime and activate the NLRP3 inflammasome that stimulates autocrine cytokine release in human mesothelial cells. Part Fibre Toxicol, 10, 39.

45 Thompson, J.K., Westbom, C.M., MacPherson, M.B., Mossman, B.T., Heintz, N.H., Spiess, P. and Shukla, A. (2014) Asbestos modulates thioredoxin-thioredoxin interacting protein interaction to regulate inflammasome activation. Part Fibre Toxicol, 11, 24.

46 Kukkonen, M.K., Vehmas, T., Piirila, P. and Hirvonen, A. (2014) Genes involved in innate immunity associated with asbestos-related fibrotic changes. Occup Environ Med, 71, 48-54.

47 Verma, D., Lerm, M., Blomgran Julinder, R., Eriksson, P., Soderkvist, P. and Sarndahl, E. (2008) Gene polymorphisms in the NALP3 inflammasome are associated with interleukin-1 production and severe inflammation: relation to common inflammatory diseases? Arthritis Rheum, 58, 888894.

48 Ji, X., Hou, Z., Wang, T., Jin, K., Fan, J., Luo, C., Chen, M., Han, R. and Ni, C. (2012) Polymorphisms in inflammasome genes and risk of coal workers' pneumoconiosis in a Chinese population. PLoS One, 7, e47949.

49 Ilavska, S., Jahnova, E., Tulinska, J., Horvathova, M., Dusinska, M., Wsolova, L., Kyrtopoulos, S.A. and Fuortes, L. (2005) Immunological monitoring in workers occupationally exposed to asbestos. Toxicology, 206, 299-308.

50 Lee, J.S., Shin, J.H., Lee, J.O., Lee, K.M., Kim, J.H. and Choi, B.S. (2010) Serum Levels of Interleukin-8 and Tumor Necrosis Factor-alpha in Coal Workers' Pneumoconiosis: One-year Follow-up Study. Saf Health Work, 1, 69-79.

51 Debets, R., Timans, J.C., Homey, B., Zurawski, S., Sana, T.R., Lo, S., Wagner, J., Edwards, G., Clifford, T., Menon, S. et al. (2001) Two novel IL-1 family members, IL-1 delta and IL-1 
epsilon, function as an antagonist and agonist of NF-kappa B activation through the orphan IL-1 receptor-related protein 2. J Immunol, 167, 1440-1446.

52 Gresnigt, M.S. and van de Veerdonk, F.L. (2013) Biology of IL-36 cytokines and their role in disease. Semin Immunol, 25, 458-465.

53 Chustz, R.T., Nagarkar, D.R., Poposki, J.A., Favoreto, S., Jr., Avila, P.C., Schleimer, R.P. and Kato, A. (2011) Regulation and function of the IL-1 family cytokine IL-1F9 in human bronchial epithelial cells. Am J Respir Cell Mol Biol, 45, 145-153.

54 Fichtner-Feigl, S., Strober, W., Kawakami, K., Puri, R.K. and Kitani, A. (2006) IL-13 signaling through the IL-13alpha2 receptor is involved in induction of TGF-beta1 production and fibrosis. Nat Med, 12, 99-106.

55 Jiang, D., Liang, J., Campanella, G.S., Guo, R., Yu, S., Xie, T., Liu, N., Jung, Y., Homer, R., Meltzer, E.B. et al. (2010) Inhibition of pulmonary fibrosis in mice by CXCL10 requires glycosaminoglycan binding and syndecan-4. J Clin Invest, 120, 2049-2057.

56 Pryhuber, G.S., Huyck, H.L., Staversky, R.J., Finkelstein, J.N. and O'Reilly, M.A. (2000) Tumor necrosis factor-alpha-induced lung cell expression of antiapoptotic genes TRAF1 and cIAP2. Am J Respir Cell Mol Biol, 22, 150-156.

57 Chada, S., Bocangel, D., Ramesh, R., Grimm, E.A., Mumm, J.B., Mhashilkar, A.M. and Zheng, M. (2005) mda-7/IL24 kills pancreatic cancer cells by inhibition of the Wnt/PI3K signaling pathways: identification of IL-20 receptor-mediated bystander activity against pancreatic cancer. Mol Ther, 11, 724-733.

58 Sieger, K.A., Mhashilkar, A.M., Stewart, A., Sutton, R.B., Strube, R.W., Chen, S.Y., Pataer, A., Swisher, S.G., Grimm, E.A., Ramesh, R. et al. (2004) The tumor suppressor activity of MDA7/IL-24 is mediated by intracellular protein expression in NSCLC cells. Mol Ther, 9, 355-367.

59 Janssen, Y.M., Marsh, J.P., Absher, M.P., Hemenway, D., Vacek, P.M., Leslie, K.O., Borm, P.J. and Mossman, B.T. (1992) Expression of antioxidant enzymes in rat lungs after inhalation of asbestos or silica. J Biol Chem, 267, 10625-10630.

60 Zhang, J.W., Lv, G.C., Yao, J.M. and Hong, X.P. (2010) Assessment of serum antioxidant status in patients with silicosis. $J$ Int Med Res, 38, 884-889.

61 Franko, A., Dodic-Fikfak, M., Arneric, N. and Dolzan, V. (2009) Manganese and extracellular superoxide dismutase polymorphisms and risk for asbestosis. J Biomed Biotechnol, 2009, 493083.

62 Baldys, A., Pande, P., Mosleh, T., Park, S.H. and Aust, A.E. (2007) Apoptosis induced by crocidolite asbestos in human lung epithelial cells involves inactivation of Akt and MAPK pathways. Apoptosis, 12, 433-447.

63 Mossman, B.T., Lounsbury, K.M. and Reddy, S.P. (2006) Oxidants and signaling by mitogenactivated protein kinases in lung epithelium. Am J Respir Cell Mol Biol, 34, 666-669.

64 Tamminen, J.A., Myllarniemi, M., Hyytiainen, M., Keski-Oja, J. and Koli, K. (2012) Asbestos exposure induces alveolar epithelial cell plasticity through MAPK/Erk signaling. J Cell Biochem, 113, 2234-2247.

65 Pati, D. (2008) Oncogenic activity of separase. Cell Cycle, 7, 3481-3482. 
66 Galang, C.K., Muller, W.J., Foos, G., Oshima, R.G. and Hauser, C.A. (2004) Changes in the expression of many Ets family transcription factors and of potential target genes in normal mammary tissue and tumors. J Biol Chem, 279, 11281-11292.

67 Catalano, A., Caprari, P., Rodilossi, S., Betta, P., Castellucci, M., Casazza, A., Tamagnone, L. and Procopio, A. (2004) Cross-talk between vascular endothelial growth factor and semaphorin3 A pathway in the regulation of normal and malignant mesothelial cell proliferation. Faseb J, 18, 358-360.

68 Serini, G., Maione, F., Giraudo, E. and Bussolino, F. (2009) Semaphorins and tumor angiogenesis. Angiogenesis, 12, 187-193.

69 Shukla, A., Bosenberg, M.W., MacPherson, M.B., Butnor, K.J., Heintz, N.H., Pass, H.I., Carbone, M., Testa, J.R. and Mossman, B.T. (2009) Activated cAMP response element binding protein is overexpressed in human mesotheliomas and inhibits apoptosis. Am J Pathol, 175, 21972206.

70 Gerstel, D., Wegwitz, F., Jannasch, K., Ludewig, P., Scheike, K., Alves, F., Beauchemin, N., Deppert, W., Wagener, C. and Horst, A.K. CEACAM1 creates a pro-angiogenic tumor microenvironment that supports tumor vessel maturation. Oncogene, 30, 4275-4288.

71 Gamage, N., Barnett, A., Hempel, N., Duggleby, R.G., Windmill, K.F., Martin, J.L. and McManus, M.E. (2006) Human sulfotransferases and their role in chemical metabolism. Toxicol $\mathrm{Sci}, \mathbf{9 0}, 5-22$.

72 Anttila, S., Raunio, H. and Hakkola, J. (2011) Cytochrome P450-mediated pulmonary metabolism of carcinogens: regulation and cross-talk in lung carcinogenesis. Am J Respir Cell Mol Biol, 44, 583-590.

73 Lorand, L. and Graham, R.M. (2003) Transglutaminases: crosslinking enzymes with pleiotropic functions. Nat Rev Mol Cell Biol, 4, 140-156.

74 Aoki, F., Kurabayashi, M., Hasegawa, Y. and Kojima, I. (2005) Attenuation of bleomycininduced pulmonary fibrosis by follistatin. Am J Respir Crit Care Med, 172, 713-720.

75 de Kretser, D.M., O'Hehir, R.E., Hardy, C.L. and Hedger, M.P. (2011) The roles of activin A and its binding protein, follistatin, in inflammation and tissue repair. Mol Cell Endocrinol.

76 Aust, A.E., Cook, P.M. and Dodson, R.F. (2011) Morphological and chemical mechanisms of elongated mineral particle toxicities. $J$ Toxicol Environ Health B Crit Rev, 14, 40-75.

77 Ghio, A.J., Kennedy, T.P., Whorton, A.R., Crumbliss, A.L., Hatch, G.E. and Hoidal, J.R. (1992) Role of surface complexed iron in oxidant generation and lung inflammation induced by silicates. Am J Physiol, 263, L511-518.

78 Chao, C.C., Lund, L.G., Zinn, K.R. and Aust, A.E. (1994) Iron mobilization from crocidolite asbestos by human lung carcinoma cells. Arch Biochem Biophys, 314, 384-391.

79 Chao, C.C., Park, S.H. and Aust, A.E. (1996) Participation of nitric oxide and iron in the oxidation of DNA in asbestos-treated human lung epithelial cells. Arch Biochem Biophys, 326, 152-157. 
80 Gkouvatsos, K., Papanikolaou, G. and Pantopoulos, K. (2011) Regulation of iron transport and the role of transferrin. Biochim Biophys Acta.

81 Wang, X., Wu, Y., Stonehuerner, J.G., Dailey, L.A., Richards, J.D., Jaspers, I., Piantadosi, C.A. and Ghio, A.J. (2006) Oxidant generation promotes iron sequestration in BEAS-2B cells exposed to asbestos. Am J Respir Cell Mol Biol, 34, 286-292.

82 Lindahl, M., Ekstrom, T., Sorensen, J. and Tagesson, C. (1996) Two dimensional protein patterns of bronchoalveolar lavage fluid from non-smokers, smokers, and subjects exposed to asbestos. Thorax, 51, 1028-1035.

83 Kim, H.J., Lee, J.S., Kim, J.D., Cha, H.J., Kim, A., Lee, S.K., Lee, S.C., Kwon, B.S., Mittler, R.S., Cho, H.R. et al. (2012) Reverse signaling through the costimulatory ligand CD137L in epithelial cells is essential for natural killer cell-mediated acute tissue inflammation. Proc Natl Acad Sci U S A, 109, E13-22.

84 Giordano, G., van den Brule, S., Lo Re, S., Triqueneaux, P., Uwambayinema, F., Yakoub, Y., Couillin, I., Ryffel, B., Michiels, T., Renauld, J.C. et al. (2010) Type I interferon signaling contributes to chronic inflammation in a murine model of silicosis. Toxicol Sci, 116, 682-692.

85 Mantovani, A., Valentino, S., Gentile, S., Inforzato, A., Bottazzi, B. and Garlanda, C. (2013) The long pentraxin PTX3: a paradigm for humoral pattern recognition molecules. Ann N Y Acad Sci, 1285, $1-14$.

86 Herseth, J.I., Volden, V., Schwarze, P.E., Lag, M. and Refsnes, M. (2008) IL-1beta differently involved in IL-8 and FGF-2 release in crystalline silica-treated lung cell co-cultures. Part Fibre Toxicol, $\mathbf{5}, 16$.

87 Hayashi, H., Maeda, M., Murakami, S., Kumagai, N., Chen, Y., Hatayama, T., Katoh, M., Miyahara, N., Yamamoto, S., Yoshida, Y. et al. (2009) Soluble interleukin-2 receptor as an indicator of immunological disturbance found in silicosis patients. Int $J$ Immunopathol Pharmacol, 22, 53-62.

88 Piguet, P.F. and Vesin, C. (1994) Treatment by human recombinant soluble TNF receptor of pulmonary fibrosis induced by bleomycin or silica in mice. Eur Respir J, 7, 515-518.

89 Braz, N.F., Carneiro, A.P., Amorim, M.R., de Oliveira Ferreira, F., Lacerda, A.C., Silva de Miranda, A., Teixeira, M.M., Teixeira, A.L. and Mendonca, V.A. (2014) Association between inflammatory biomarkers in plasma, radiological severity, and duration of exposure in patients with silicosis. J Occup Environ Med, 56, 493-497.

90 Hubbard, A.K., Timblin, C.R., Shukla, A., Rincon, M. and Mossman, B.T. (2002) Activation of NF-kappaB-dependent gene expression by silica in lungs of luciferase reporter mice. Am J Physiol Lung Cell Mol Physiol, 282, L968-975.

91 van Berlo, D., Knaapen, A.M., van Schooten, F.J., Schins, R.P. and Albrecht, C. (2010) NFkappaB dependent and independent mechanisms of quartz-induced proinflammatory activation of lung epithelial cells. Part Fibre Toxicol, 7, 13.

92 Morris, G.F. (2010) An alternative to lung inflammation and fibrosis. Am J Pathol, 176, 25952598 . 
93 Yang, L., Cui, H., Wang, Z., Zhang, B., Ding, J., Liu, L. and Ding, H.F. (2010) Loss of negative feedback control of nuclear factor-kappaB2 activity in lymphocytes leads to fatal lung inflammation. Am J Pathol, 176, 2646-2657.

94 Tully, J.E., Hoffman, S.M., Lahue, K.G., Nolin, J.D., Anathy, V., Lundblad, L.K., Daphtary, N., Aliyeva, M., Black, K.E., Dixon, A.E. et al. (2013) Epithelial NF-kappaB orchestrates house dust mite-induced airway inflammation, hyperresponsiveness, and fibrotic remodeling. $J$ Immunol, 191, 5811-5821.

95 Tully, J.E., Nolin, J.D., Guala, A.S., Hoffman, S.M., Roberson, E.C., Lahue, K.G., van der Velden, J., Anathy, V., Blackwell, T.S. and Janssen-Heininger, Y.M. (2012) Cooperation between classical and alternative NF-kappaB pathways regulates proinflammatory responses in epithelial cells. Am J Respir Cell Mol Biol, 47, 497-508.

96 Guo, Y., Xiao, L., Sun, L. and Liu, F. (2012) Wnt/beta-catenin signaling: a promising new target for fibrosis diseases. Physiol Res, 61, 337-346.

97 Konigshoff, M. (2011) Lung cancer in pulmonary fibrosis: tales of epithelial cell plasticity. Respiration, 81, 353-358.

98 Campbell, W.J., Huggins, C.W., Wylie, A.G. (1980), In U.S. Bur. Mines, Rep. Invest. NIEHS, Vol. 8452.

99 Verhees, K.J., Schols, A.M., Kelders, M.C., Op den Kamp, C.M., van der Velden, J.L. and Langen, R.C. (2011) Glycogen synthase kinase-3beta is required for the induction of skeletal muscle atrophy. Am J Physiol Cell Physiol, 301, C995-C1007.

100 Sabo-Attwood, T., Ramos-Nino, M., Bond, J., Butnor, K.J., Heintz, N., Gruber, A.D., Steele, C., Taatjes, D.J., Vacek, P. and Mossman, B.T. (2005) Gene expression profiles reveal increased mClca3 (Gob5) expression and mucin production in a murine model of asbestos-induced fibrogenesis. Am J Pathol, 167, 1243-1256.

101 Shukla, A., Lounsbury, K.M., Barrett, T.F., Gell, J., Rincon, M., Butnor, K.J., Taatjes, D.J., Davis, G.S., Vacek, P., Nakayama, K.I. et al. (2007) Asbestos-induced peribronchiolar cell proliferation and cytokine production are attenuated in lungs of protein kinase C-delta knockout mice. Am J Pathol, 170, 140-151.

102 Gentleman, R.C., Carey, V.J., Bates, D.M., Bolstad, B., Dettling, M., Dudoit, S., Ellis, B., Gautier, L., Ge, Y., Gentry, J. et al. (2004) Bioconductor: open software development for computational biology and bioinformatics. Genome Biol, 5, R80.

103 Arijs, I., Li, K., Toedter, G., Quintens, R., Van Lommel, L., Van Steen, K., Leemans, P., De Hertogh, G., Lemaire, K., Ferrante, M. et al. (2009) Mucosal gene signatures to predict response to infliximab in patients with ulcerative colitis. Gut, 58, 1612-1619.

104 Irizarry, R.A., Hobbs, B., Collin, F., Beazer-Barclay, Y.D., Antonellis, K.J., Scherf, U. and Speed, T.P. (2003) Exploration, normalization, and summaries of high density oligonucleotide array probe level data. Biostatistics, 4, 249-264.

105 Benjamini, Y. and Hochberg, Y. (1995) Controlling the False Discovery Rate - a Practical and Powerful Approach to Multiple Testing. J Roy Stat Soc B Met, 57, 289-300. 


\section{SUPPLEMENTAL MATERIAL}

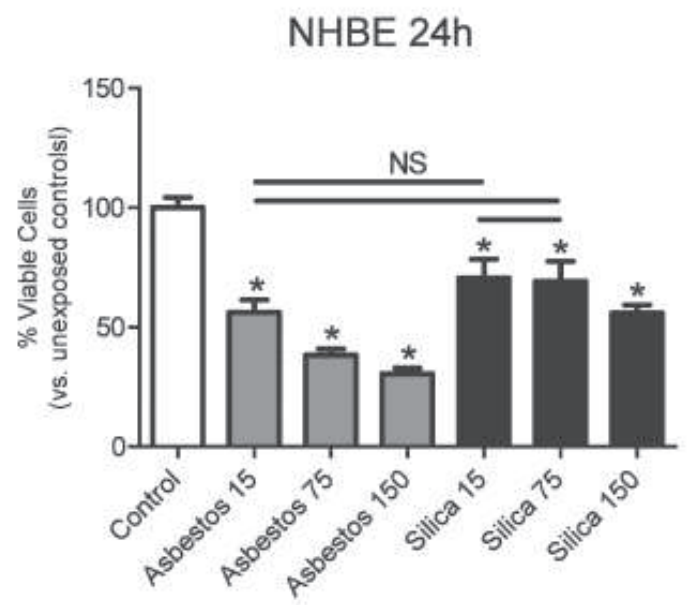

Supplemental figure S1: Effects of mineral exposure on $\mathrm{NHBE}$ cell viability (24h). Cell viability as determined by the trypan blue exclusion assay. NHBE cells were exposed to 3 equal surface area doses $\left(x 10^{6} \mathrm{\mu m}^{2} / \mathrm{cm}^{2}\right.$ ) (respectful mass-based doses below) for 24h. Subsequently cells were collected and viability determined (viable cells unstained). Values are represented as the percent viable cells vs. unexposed controls. For each condition, $N=3$ and results represent pooled data from two independent experiments (due to limited life-span of primary cells). (*) $p<0.05$ vs. unexposed controls.

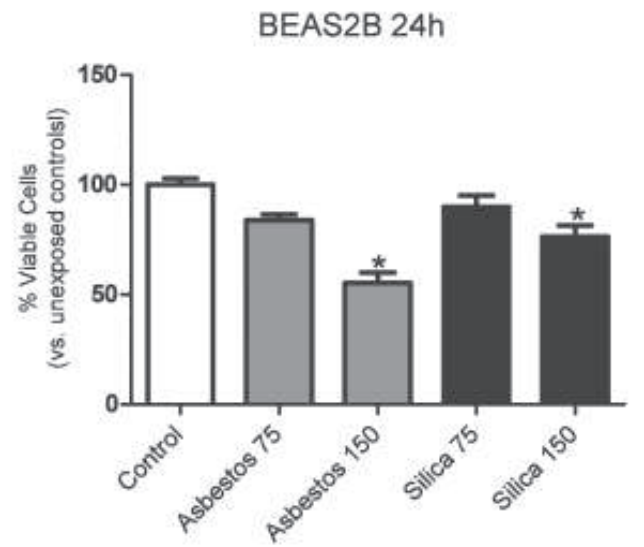

Supplemental figure S2: Effects of mineral exposure on BEAS2B cell viability (24h). Cell viability as determined by the trypan blue exclusion assay. NHBE cells were exposed to 2 equal surface area doses $\left(x 10^{6} \mu^{2} / \mathrm{cm}^{2}\right)$ for $24 \mathrm{~h}$. Subsequently cells were collected and viability determined (viable cells unstained). Values are represented as the percent viable cells vs. unexposed controls. For each 
condition $N=3$ and results are representative of 3 independent experiments. (*) $p<0.05 v$ s. unexposed controls.
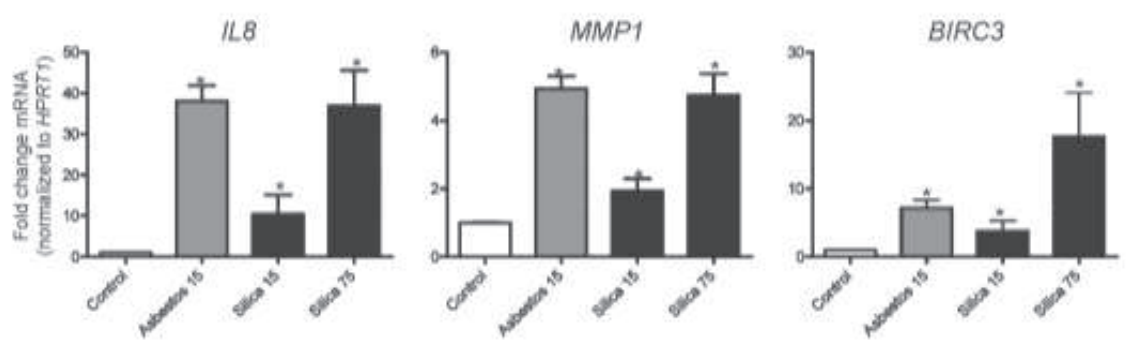

Supplemental figure S3: Confirmation of microarray findings in NHBE cell by QRT-PCR. NHBE cells were exposed to asbestos and silica (same doses as microarray) in an independent experiment (24h). Steady-state mRNA levels were determined by QRT-PCR for (A) IL8, (B) MMP1, and (C) BIRC3. Expression levels were normalized to the housekeeping gene, HPRT (Hypoxanthine phosphoribosyltransferase 1). Values represented are the mean $\pm S E M . N=3$, (*) $p<0.05$.

\section{Supplemental table legends (the supplementary tables are available online via the journal website):}

Supplemental table S1: Probe-sets significantly altered by asbestos (15) vs. unexposed controls. List of all probe-sets significantly altered by asbestos (15) in NHBE cells (24h). List includes Probe-set ID (Affymetrix ID), Entrez Gene Name, Gene Symbol, Fold-change value, and adj.p-value. Probe-sets are listed in order of decreasing fold-change value (highest expression to lowest expression. Threshold cut-off of 1.5-fold vs. controls with an adj.p $<0.05$.

Supplemental table S2: Probe-sets significantly altered by silica (15) vs. unexposed controls. List of all probe-sets significantly altered by silica (15) in NHBE cells (24h). List includes Probe-set ID (Affymetrix ID), Entrez Gene Name, Gene Symbol, Fold-change value, and adj.p-value. Probe-sets are listed in order of decreasing fold-change value (highest expression to lowest expression. Threshold cut-off of 1.5-fold vs. controls with an adj.p $<0.05$.

Supplemental table S3: Probe-sets significantly altered by silica (75) vs. unexposed controls. List of all probe-sets significantly altered by Silica (75) in NHBE cells (24h). List includes Probe-set ID (Affymetrix ID), Entrez Gene Name, Gene Symbol, Fold-change value, and adj.p-value. Probe-sets are listed in order of decreasing fold-change value (highest expression to lowest expression. Threshold cut-off of 1.5-fold vs. controls with an adj.p $<0.05$.

Supplemental table S4: Probe-sets commonly altered by asbestos (15) and silica (75). List of probesets significantly altered by both asbestos (15) and silica (75) in NHBE cells (249 probe-sets; 198 genes). Probe-sets are listed in order of decreasing fold-change value (asbestos (15) vs. unexposed controls). Threshold cut-off of 1.5-fold vs. controls with an adj.p $<0.05$. + Fold-change values for asbestos (15) vs. unexposed controls. 


\title{
CHAPTER 4
}

\section{DIFFERENCES IN GENE EXPRESSION AND CYTOKINE PRODUCTION BY CRYSTALLINE VS. AMORPHOUS SILICA IN HUMAN LUNG EPITHELIAL CELLS}

\author{
Timothy N Perkins ${ }^{1}$, Arti Shukla ${ }^{1}$, Paul M Peeters ${ }^{2}$, Jeremy L Steinbacher ${ }^{3}$, Christopher C Landry ${ }^{3}$, \\ Sherrill L Macura ${ }^{1}$ Chad Steele ${ }^{4}$, Niki L Reynaert ${ }^{2}$, Emiel FM Wouters ${ }^{2}$, Brooke T Mossman ${ }^{1}$ \\ ${ }^{1}$ Department of Pathology, University of Vermont College of Medicine, 89 Beaumont Avenue, Burlington, VT \\ 05405, USA \\ ${ }^{2}$ Department of Respiratory Medicine, Maastricht University Medical Centre, Maastricht University, Maastricht, \\ The Netherlands. \\ ${ }^{3}$ Department of Chemistry, University of Vermont, Burlington, VT 05405, USA \\ ${ }^{4}$ Department of Medicine, University of Alabama at Birmingham School of Medicine, Birmingham, AL \\ 35233,USA
}

\section{Particle and Fibre Toxicology. 2012 Feb 2;9(1):6.}

\begin{abstract}
Background: Exposure to respirable crystalline silica particles, as opposed to amorphous silica, is associated with lung inflammation and pulmonary fibrosis (silicosis). We used Affymetrix/GeneSifter microarray analysis to determine whether gene expression profiles differed in a human bronchial epithelial cell line (BEAS 2B) exposed to cristobalite vs. amorphous silica particles at non-toxic and equal surface areas $\left(75\right.$ and $\left.150 \times 10^{6} \mu \mathrm{m}^{2} / \mathrm{cm}^{2}\right)$. Bioplex analysis also was used to determine profiles of secreted cytokines and chemokines in response to both particles. Finally, primary human bronchial epithelial cells (NHBE) were used to comparatively assess silica particle-induced alterations in gene expression.
\end{abstract}

Results: Microarray analysis at 24 hours in BEAS 2B revealed 333 and 631 significant alterations in gene expression induced by cristobalite at low (75) and high (150 X $10^{6} \mu \mathrm{m}^{2} / \mathrm{cm}^{2}$ ) amounts, respectively ( $\mathrm{p}<0.05 /$ cut off $\geq 2.0$-fold change). Exposure to amorphous silica micro-particles at high amounts $\left(150 \times 10^{6} \mu \mathrm{m}^{2} / \mathrm{cm}^{2}\right)$ induced 108 significant gene changes. Bioplex analysis of 27 human cytokines and chemokines revealed 9 secreted mediators $(\mathrm{p}<0.05)$ induced by crystalline silica, but none were induced by amorphous silica. QRT-PCR revealed that cristobalite selectively up-regulated stress-related genes and cytokines (FOS, ATF3, IL6 and IL8) early and over time (2, 4, 8, and $24 \mathrm{~h}$ ). Patterns of gene expression in NHBE cells were similar overall to BEAS 2B cells. At $75 \times 10^{6} \mu \mathrm{m}^{2} / \mathrm{cm}^{2}$, there were 339 significant alterations in gene expression induced by cristobalite and 42 by amorphous silica. Comparison of genes in response to cristobalite $\left(75 \times 10^{6} \mu \mathrm{m}^{2} / \mathrm{cm}^{2}\right)$ revealed 60 common, significant gene alterations in NHBE and BEAS $2 \mathrm{~B}$ cells.

Conclusion: Cristobalite silica, as compared to synthetic amorphous silica particles at equal surface area concentrations, had comparable effects on the viability of human bronchial 
epithelial cells. However, effects on gene expression, as well as secretion of cytokines and chemokines, drastically differed, as the crystalline silica induced more intense responses. Our studies indicate that toxicological testing of particulates by surveying viability and/or metabolic activity is insufficient to predict their pathogenicity. Moreover, they show that acute responses of the lung epithelium, including up-regulation of genes linked to inflammation, oxidative stress, and proliferation, as well as secretion of inflammatory and proliferative mediators, can be indicative of pathologic potential using either immortalized lines (BEAS 2B) or primary cells (NHBE). Assessment of the degree and magnitude of these responses in vitro are predictive in determining the pathogenicity of potentially harmful particulates. 


\section{BACKGROUND}

Occupational and environmental exposure to fine and ultrafine particulates is rapidly becoming an overwhelming area of concern. With increasing numbers and compositional heterogeneity of potentially harmful natural and synthetic particulates, there is a vital need for screening assays to determine their potential pathogenicity. Crystalline silica particles are known to cause silicosis (a pneumoconiosis) and have other detrimental respiratory effects when inhaled in excessive amounts [1]. Airborne exposures are also associated with lung inflammatory diseases, increased susceptibility to infection, as well as lung cancers, especially in smokers $[2,3]$. Crystalline silica was stated to be a Class I carcinogen (IARC 1997), though epidemiologic data are inconsistent, and its carcinogenic potential in nonsmokers remains controversial [4]. Exposure to crystalline silica is associated with industries and occupations including, sandblasting, ceramics, cement manufacture, construction, and quarry and foundry workers [2, 5-8]. Although crystalline silica exists in many different polymorphs, those of particular concern are the naturally occurring polymorphs quartz, cristobalite and tridymite [9]. Cristobalite was used in studies here because of its higher bioreactivity when compared to quartz in a rat inhalation model [10].

Inhalation of crystalline silica or other pathogenic minerals, such as asbestos and airborne particulate matter (PM), results in lung injury by mechanisms which are not well understood $[11,12]$. Crystalline silica particles induce extensive inflammatory and proliferative effects in vitro and in vivo [13-19]. Activation of several signalling pathways, including the mitogenactivated protein kinases (MAPKs) [20-22] as well as transcription factors including activator protein-1 (AP-1) [23] and nuclear factor kappa $\mathrm{B}(\mathrm{NF \kappa B})$, are thought to contribute to the proinflammatory, toxic and mitogenic effects of silica [24]. Understanding the broad spectrum of initial events induced by particulate-cell interactions is crucial to revealing the molecular mechanisms that contribute to inflammation, abnormal proliferation and cross-talk between epithelial cells and other cell types in the lung.

Here we focused on lung epithelial cells, which initially interact with respirable particles after inhalation to provide insight into molecular events initiating silica-induced disease processes. Since surface area, as opposed to equal mass concentration, has been shown to be a more appropriate parameter of dosing to determine the effects of fine and ultrafine particulates in biological systems [25-28], human bronchial epithelial cells were exposed to crystalline silica (cristobalite) or amorphous silica (synthetic, dense silica spheres) at non-toxic and equal surface area concentrations. As previous studies from our group have shown that the magnitude and duration of gene expression alterations in human mesothelial cells by asbestos fibers may be predictive of their pathogenic potential [29-31], we utilized robust microarray profiling to compare responses of human lung epithelial cells to pathogenic crystalline vs. amorphous silica. Our findings link the extent of changes in gene expression and cytokine production to silica crystallinity and pathogenicity in silicosis. 


\section{RESULTS AND DISCUSSION}

\section{Cristobalite and amorphous silica particles have equal effects on viability of BEAS $2 B$} cells

Surface area concentration is a more relevant dosing parameter than equivalent weight comparisons of particles as the biological activity of particles is largely based on their respective surface characteristics [28, 32-34]. Much of this is accredited to the ability of pathogenic particles to produce reactive oxygen and nitrogen species (ROS and RNS) [35] that promote inflammatory responses and cellular injury [36]. The surface areas of cristobalite and amorphous silica particles (dense silica micro-spheres) were determined by BET nitrogen adsorption analysis [31]. Table 1 presents the surface area/unit mass $\left(\mathrm{m}^{2} / \mathrm{g}\right)$ and other properties of cristobalite and amorphous silica particles, including chemical composition $\left(\mathrm{SiO}_{2}\right)$, which is confirmed in supplementary figure 1 by SEM-EDS analysis. To avoid use of lethal concentrations of particles, we first performed cell viability assays over a range of concentrations. Both particles had dose-responsive effects on viability, inducing only about $15 \%$ loss of viability (non-significant) at highest concentrations $\left(150 \times 10^{6} \mu \mathrm{m} 2 / \mathrm{cm} 2\right)$ over a $24 \mathrm{~h}$ period (Figure 1). Both a low $\left(75 \times 10^{6} \mu \mathrm{m} 2 / \mathrm{cm} 2\right)$ and high dose $\left(150 \times 10^{6} \mu \mathrm{m} 2 / \mathrm{cm} 2\right)$ of crystalline silica particles and a high dose $\left(150 \times 10^{6} \mu \mathrm{m} 2 / \mathrm{cm} 2\right)$ of amorphous silica were evaluated by microarray and Bioplex analysis for gene expression and cytokine/chemokine secretion, respectively.

Table 1: Silica particle characterization. This table summarizes silica particle characteristic of cristobalite and amorphous silica particles used in all experiments, presenting from left to right, the particle name, chemical composition, surface area, mean diameter and the source of the particles.

\begin{tabular}{lllcl}
\hline Particle & $\begin{array}{l}\text { Chemical } \\
\text { Comp. }\end{array}$ & $\begin{array}{l}\text { S.A. } \\
\left(\mathbf{m}^{2} / \mathbf{g}\right)^{\mathbf{a}}\end{array}$ & $\begin{array}{l}\text { Mean size } \\
(\boldsymbol{\mu m})^{\mathbf{b}}\end{array}$ & Source $\dagger$ \\
\hline Cristobalite & $\mathrm{SiO}_{2}$ & 5.1 & $1.5 \pm 2.6$ & C\&E Mineral Corp, King of Prussia, PA \\
Amorphous & $\mathrm{SiO}_{2}$ & 2.2 & $3.5 \pm 0.3$ & University of Vermont, Dept. of Chemistry \\
\hline
\end{tabular}

${ }^{\mathrm{a}}$ S.A. $=$ Surface $\operatorname{area} / \operatorname{mass}\left(\mathrm{m}^{2} / \mathrm{g}\right)$.

${ }^{\mathbf{b}}$ Mean particle diameter \pm geometric standard deviation

$\dagger$ See sources for description of cristobalite [75] and amorphous silica particles described in Methods and [74]. 
A

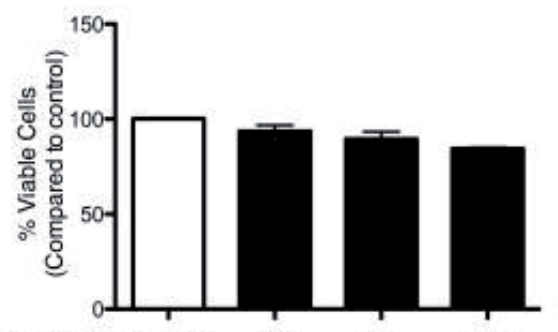

$\left(x 10^{6} \mu \mathrm{m}^{2} / \mathrm{cm}^{2}\right)^{\text {a }}$ Control

\begin{tabular}{ccccc}
$\left(\mathrm{x} 10^{6} \mu \mathrm{m}^{2} / \mathrm{cm}^{2}\right)^{\mathrm{a}}$ & Control & 15 & 75 & 150 \\
\hline$\left(\mu \mathrm{g} / \mathrm{cm}^{2}\right)^{\mathrm{b}}$ & Control & 2.94 & 14.71 & 29.4 \\
\hline
\end{tabular}

B

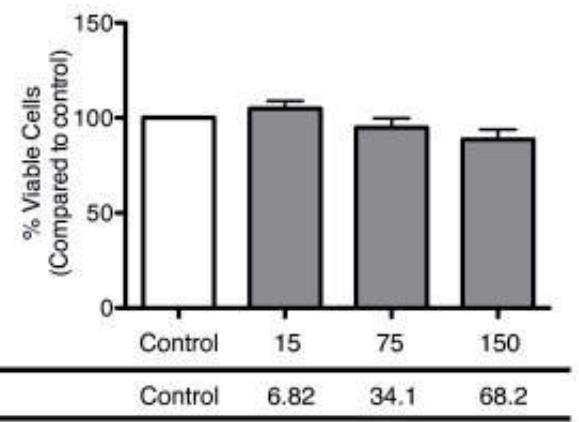

s Surface area concentration

b Mass concentration

$\square$ Control

Cristobalite silica

$\square$ Amorphous silica

Figure 1: Assessment of BEAS $2 B$ cell viability after exposure to silica particles. Cell viability assessed by the Trypan blue exclusion assay of cells exposed to cristobalite (A) and amorphous silica particles (B) for $24 \mathrm{~h}$. Results are expressed as the mean percent viable cells compared to unexposed controls \pm SEM and are representative of 3 independent experiments $(N=3$ for each group in each experiment). *Significantly different from controls $(P<0.05) .{ }^{a}$ denotes the surface area concentrations expressed as ( $\left.X 10^{6} \mu \mathrm{m} 2 / \mathrm{cm} 2\right)$ and ${ }^{b}$ denotes the respective mass concentrations of particles used $\left(\mu \mathrm{g} / \mathrm{cm}^{2}\right)$. Concentrations presented are represented by surface area for all other figures and tables.

\section{Cristobalite and amorphous silica particles interact with and are taken up by BEAS 2B cells}

To determine whether both particle types were taken up by lung epithelial cells, scanning electron micrographs (SEM) of BEAS 2B cells exposed to silica particles $\left(75 \times 10^{6}\right.$ $\mu \mathrm{m} 2 / \mathrm{cm} 2$ ) were examined at 2 and $24 \mathrm{~h}$ (Figure 2). The presence of silica particles was confirmed by electron dispersion spectroscopy (EDS) analysis, in which a spectrum was taken of a point "on-particle" showing a strong silicon ( $\mathrm{Si}$ ) peak and an "off-particle" spectrum, which contained no (Si) peak. Both cristobalite and amorphous silica particles interacted with cells at $2 \mathrm{~h}$ and were internalized by $24 \mathrm{~h}$ (Figure 2). No major toxic effects were observed, correlating with results of cell viability studies. Based on these results, we investigated alterations in gene expression and mediator secretion at these non-toxic particle doses. 


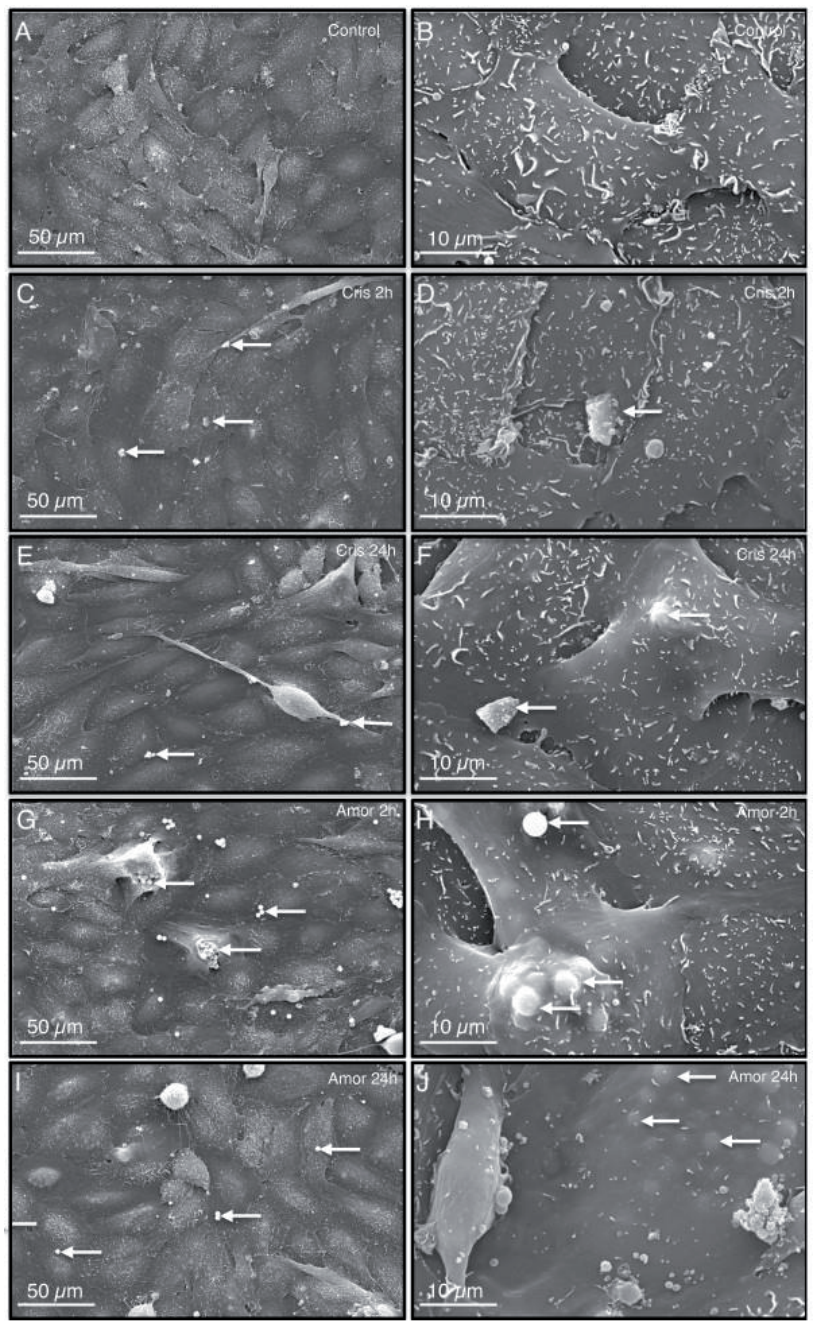

Figure 2: Interaction and uptake of silica particles by BEAS $2 B$ cells. Scanning Electron Micrographs (SEM) of BEAS $2 B$ cells Unexposed controls $(\boldsymbol{A}, \boldsymbol{B})$, Exposed to Cristobalite $\left(75 \times 10^{6}\right.$ $\mu \mathrm{m} 2 / \mathrm{cm} 2)$ for $2 \mathrm{~h}(\boldsymbol{C}, \boldsymbol{D}), 24 \mathrm{~h}(\boldsymbol{E}, \boldsymbol{F})$ and amorphous silica $\left(75 \times 10^{6} \mu \mathrm{m} 2 / \mathrm{cm} 2\right)$ for $2 \mathrm{~h}(\boldsymbol{G}, \boldsymbol{H})$ and $24 \mathrm{~h}$ $(I, J)$. Panels on the left are at low magnification $(500 X)$ scale bar $=50 \mu m$ and on the right at high magnification $(2500 X)$ scale bar $=10 \mu \mathrm{m}$. White arrows indicate silica particles.

Microarray analysis reveals robust changes in gene expression profiles in BEAS 2B cells exposed to cristobalite vs. amorphous silica particles

It has been shown both in vitro and in vivo that crystalline silica particles are more biologically active and pathogenic than amorphous silica particles [19, 37-39]. Therefore, we sought to determine if there were significant differences in how different silica particles (crystalline vs. amorphous) would affect gene expression and mediator secretion at equal surface area concentrations in the BEAS 2B immortalized cell line. Affymetrix/GeneSifter 
analysis of BEAS $2 \mathrm{~B}$ cells exposed to cristobalite silica at 75 and $150 \times 10^{6} \mu \mathrm{m} 2 / \mathrm{cm} 2$ were compared to patterns using amorphous silica particles at $150 \times 10^{6} \mu \mathrm{m} 2 / \mathrm{cm} 2$ for $24 \mathrm{~h}$, the time point of maximum gene expression observed with pathogenic particulates in previous studies [29-31]. A dose-responsive pattern was observed with cristobalite silica that induced a total of 333 and 631 significant gene changes at 75 and $150 \times 10^{6} \mu \mathrm{m} 2 / \mathrm{cm} 2$, respectively ( $\mathrm{p}<0.05$ with a cut-off of $\geq 2.0$-fold change compared to controls). In addition, expression of certain genes was dose-responsive as well. For instance, matrix metallopeptidase 1 (MMP1) gene expression was increased 16-fold and 44-fold at 75 and $150 \times 10^{6} \mu \mathrm{m} 2 / \mathrm{cm} 2$, respectively (Table 2). This dose responsive trend was observed overall in mRNA levels of the 10 most highly expressed genes after cristobalite exposures as presented in Table 2. However, amorphous silica particles, at the highest surface area concentration $\left(150 \times 10^{6}\right.$ $\mu \mathrm{m} 2 / \mathrm{cm} 2$ ), induced only 108 significant gene changes, nearly 6-fold less than cristobalite silica (Figure 3A). Of the 108 changes induced by amorphous silica, 93 of these changes were also induced by cristobalite silica at the same concentration.

Gene ontology (GO) analysis then was used to categorize changes in gene expression caused by silica particle exposures (Figure 3B-D). Ten categories of interest (cell signalling, cell adhesion, cell proliferation, apoptosis, regulation of programmed cell death, protein metabolic processes, immune response, cell motility, oxidation-reduction, and cell matrix adhesion) were used to classify gene changes [29-31]. Interestingly, patterns of both cristobalite silica and amorphous silica-induced mRNA expression were similar, with most gene expression alterations related to cell signalling and proliferation, apoptosis, protein metabolic processes and immune responses. Though the intensity of gene changes (both in number and magnitude or fold-change) was dose-dependent, overall the same patterns of change were seen (Supplementary figure 2). These five categories account for 77,73 and $80 \%$ of gene changes in BEAS 2B cells exposed to cristobalite at 75 and $150 \times 10^{6} \mu \mathrm{m} 2 / \mathrm{cm} 2$ and amorphous silica at $150 \times 10^{6} \mu \mathrm{m} 2 / \mathrm{cm} 2$, respectively.

Table 2 lists the top 10 gene changes induced by cristobalite vs. amorphous silica. The majority of the top 10 up-regulated genes are related to immune response (OAS1, OAS2, OASL, IFI44, IFI44L, BLNK), apoptosis (MX1, BTG2, IL24 and IFI27) cell signalling (MX2) and dissolution of extracellular matrix $(M M P 1)$. These genes may contribute to the development of silicosis or, in the case of $M M P 1$, repair of lung injury. Similar changes in gene expression were observed in a recent study investigating common gene expression responses of macrophages to a number of pathogens including toll-like receptor (TLR) agonists, Salmonella, and silica nanoparticles in which the authors described a "macrophage core response module" [40]. Common changes compared with results in Table 2 include: $M X 1, M X 2, O A S 1, O A S 2$, and IFI44. In our studies, several other genes included in this "macrophage core response module" were also altered in expression $(\mathrm{p}<0.05)$ by cristobalite including EGR1, EGR2, FOS, GADD45B, IFIT1, IFIT2, JUN, MAFB, PLAU, and PTGS1. These results indicate that foreign materials consistently affect a number of common genes in both macrophages and lung epithelial cells. The specific roles these genes play in the development of lung disease is not well understood; however, they appear to be elicited in acute responses to foreign materials and infectious agents. How these rapid responses affect epithelial cells and macrophages, respectively as well as cross-talk between these cells, is not 
understood, but they may be important, initial mechanisms of cell response to crystalline silica.

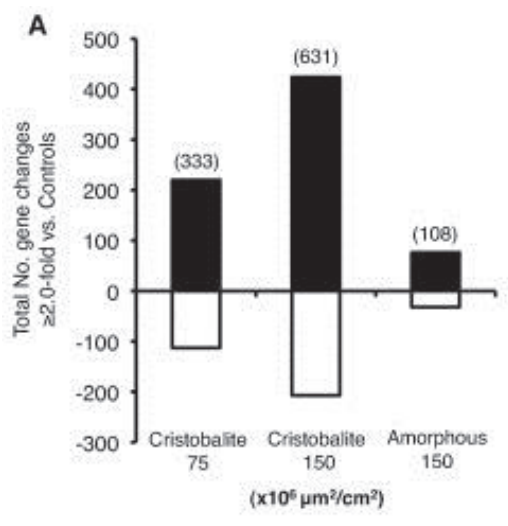

B
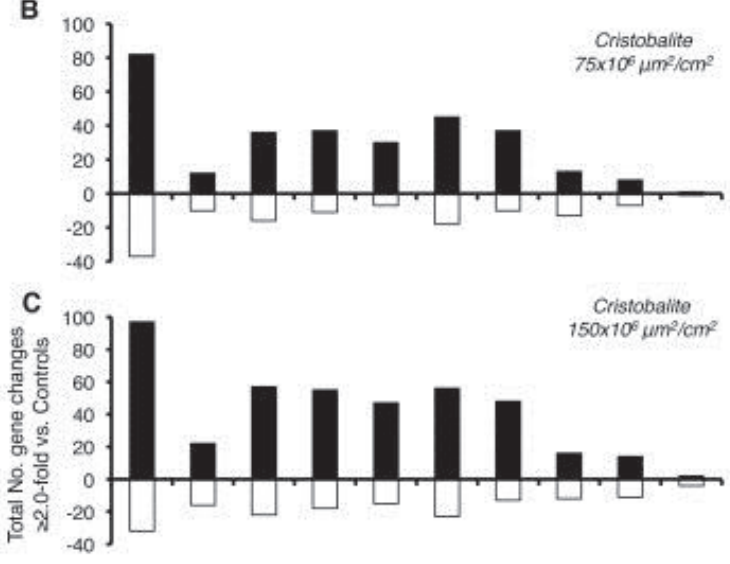

D

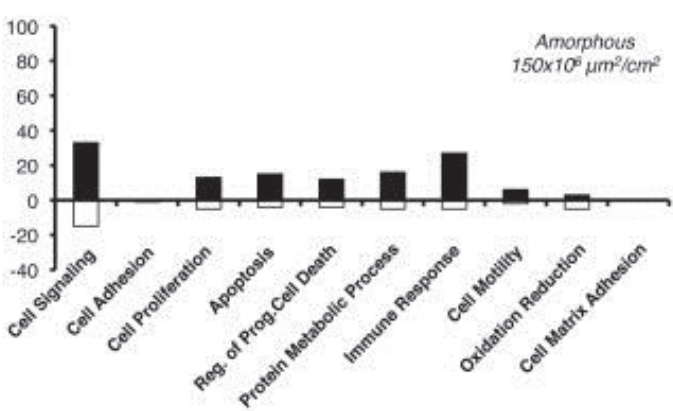

Figure 3: Microarray analysis of BEAS $2 B$ cells in response to silica particle exposure (24 h). (A) Total number of significant gene changes $(p<0.05)$ with a cut-off of $\geq 2.0$-fold change in expression compared to unexposed controls in BEAS $2 B$ exposed for $24 \mathrm{~h}$ (total number of gene changes). Gene ontology analysis of BEAS $2 B$ exposed to (B) Cristobalite at $75 \times 10^{6} \mu \mathrm{m} 2 / \mathrm{cm} 2,(\boldsymbol{C}) 150 \times 10^{6}$ $\mu \mathrm{m} 2 / \mathrm{cm} 2$ and (D) amorphous silica at $150 \times 10^{6} \mu \mathrm{m} 2 / \mathrm{cm} 2$. (A-D) positive values represent number of genes up-regulated (Black bars) and negative values represent number of gene down-regulated (white bars). 
Table 2: Top 10 genes affected by $24 \mathrm{~h}$ exposure to silica particles in BEAS 2B. Top 10 genes in $B E A S 2 B$ cells up and down-regulated by $24 \mathrm{~h}$ exposure to cristobalite silica at 75 and $150 \times 10^{6}$ $\mu^{2} / \mathrm{cm}^{2}$ and amorphous silica particles at $150 \times 10^{6} \mathrm{\mu m}^{2} / \mathrm{cm}^{2}$.

\begin{tabular}{|c|c|c|c|}
\hline \multirow[t]{3}{*}{ Gene Name (Abbreviation) } & \multicolumn{3}{|c|}{ Fold Change $^{a}$} \\
\hline & \multicolumn{2}{|c|}{ Cristobalite } & \multirow{2}{*}{$\begin{array}{c}\text { Amorphous } \\
150^{\mathrm{b}}\end{array}$} \\
\hline & $75^{\mathrm{b}}$ & $150^{b}$ & \\
\hline \multicolumn{4}{|l|}{ Up-regulated $\boldsymbol{A}$} \\
\hline Myxovirus (influenza virus) resistance 2 (mouse) (MX2) & 20.86 & 73.76 & 22.33 \\
\hline Matrix metallopeptidase 1 (interstitial collagenase) (MMP1) & 16.12 & 44.68 & 18.75 \\
\hline Sodium Channel, voltage-gated, type III, beta (SCN3B) & 15.33 & 20.54 & $\mathrm{NC}$ \\
\hline 2'-5'-oligoadenylate-synthetase 2, 69/71kDa (OAS2) & 13.56 & 34.77 & 12.17 \\
\hline $\begin{array}{l}\text { Myxovirus (influenza virus) resistance 1, interferon-inducible } \\
\text { protein p78 (mouse) (MX1) }\end{array}$ & 9.61 & 23.33 & 8.72 \\
\hline Interferon-induced protein 44-like (IFI44L) & 7.64 & 22.82 & 8.63 \\
\hline Interferon-induced protein 44 (IFI44) & 7.51 & 19.90 & 8.24 \\
\hline B-cell Linker (BLNK) & 7.24 & 9.32 & $\mathrm{NC}$ \\
\hline BTG family, member 2 (BTG2) & 7.22 & 8.56 & $\mathrm{NC}$ \\
\hline $\begin{array}{l}\text { Carcinoembryonic antigen-related cell adhesion molecule } 1 \\
\text { (CEACAM1) }\end{array}$ & 7.19 & 11.05 & $\mathrm{NC}$ \\
\hline 2'-5'-oligoadenylate synthetase 1, 40/46kDa (OAS1) & 7.09 & 20.53 & 6.75 \\
\hline Interferon, alpha-inducible protein 27 (IFI27) & 6.59 & 17.62 & 6.15 \\
\hline 2'-5'-oligoadenylate synthetase-like (OASL) & 6.80 & 18.85 & 6.92 \\
\hline Radical S-adenosyl methionine domain containing (RSAD1) & 5.45 & 23.25 & 6.09 \\
\hline Interleukin 24 (IL24) & 3.88 & 8.54 & 6.47 \\
\hline \multicolumn{4}{|l|}{ Down-regulated $\boldsymbol{\nabla}$} \\
\hline Collagen, type I, alpha 2 (COL1A2) & 6.08 & 10.86 & 2.12 \\
\hline Hypoxia inducible factor 3, alpha subunit (HIF3A) & 4.51 & 7.18 & 3.94 \\
\hline Collagen, type I, alpha 1 (COL1A1) & 4.17 & 5.52 & $\mathrm{NC}$ \\
\hline Methyltransferase like 7A (METTL7A) & 4.11 & 5.67 & 2.89 \\
\hline Cytochrome P450, family 4, subfamily B, polypeptide 1 (CYP4B1) & 3.93 & 5.55 & 3.07 \\
\hline Olfactomedin-like 3 (OLFML3) & 3.91 & 5.28 & 2.06 \\
\hline Mitogen-activated protein kinase kinase 6 (MAP2K6) & 3.86 & 4.78 & 3.21 \\
\hline Collagen, type XI, alpha 1 (COL11A1) & 3.85 & 5.46 & $\mathrm{NC}$ \\
\hline $\begin{array}{l}\text { Potassium inwardly-rectifying channel, subfamily J, member } 16 \\
\text { (KCNJ16) }\end{array}$ & 3.78 & 7.11 & 3.49 \\
\hline Cannabinoid receptor 1 (brain) (CNR1) & 3.75 & 5.44 & 3.48 \\
\hline $\begin{array}{l}\text { Calcium channel, voltage-dependent, alpha 2/delta subunit } 3 \\
\text { (CACNA2D3) }\end{array}$ & 3.53 & 3.49 & 2.65 \\
\hline Solute carrier family 38, member 4 (SLC38A4) & 3.16 & 3.76 & 2.65 \\
\hline Leucine rich repeat containing 17 (LRRC17) & 3.14 & 5.07 & 3.61 \\
\hline Protocadherin 9 (PCDH9) & 2.98 & 3.69 & 2.65 \\
\hline
\end{tabular}

${ }^{a}$ Fold change $(\mathrm{mRNA})$ expression $(\mathrm{p}<0.05)$ cut-off $\geq 2.0$-fold compared to controls $(\mathrm{n}=3)$. Top 10 genes from each exposure group.

${ }^{\mathbf{b}}$ Silica particle concentrations: ( X $\left.10^{6} \mu \mathrm{m} 2 / \mathrm{cm} 2\right) \quad \mathrm{NC}=$ No Change 


\section{Steady state mRNA levels of stress-related and inflammatory genes are induced early and selectively by cristobalite in BEAS $2 \mathrm{~B}$ cells.}

Based on the results of our microarray analyses, we investigated some genes of interest using qRT-PCR to confirm changes found in microarrays as well as to determine their time-course of induction in BEAS 2B cells. Genes investigated (up-regulated by cristobalite at 75 and 150 $\mathrm{X} 10^{6} \mu \mathrm{m} 2 / \mathrm{cm} 2$ in microarray) included the early response gene FOS (2.12, 3.51-fold), a member of the activator protein-1 (AP-1) transcription factor complex [41], activating transcription factor 3 ATF3 (2.68, 3.16-fold), which is up-regulated in gene-profiling by several particulates in human mesothelial cells [29-31, 42], and the pro-inflammatory cytokines $\operatorname{IL6}(2.12,2.59$-fold) and $\operatorname{IL8}(2.12,2.65$-fold), both shown to be up-regulated by crystalline silica in A549 cells and a number of in vitro and in vivo models [20, 43-47].

In all cases, mRNA levels of these genes were only up-regulated by crystalline in contrast to amorphous silica (Figure 4). Consistent with its role as an early response gene, FOS expression was significantly increased at $2 \mathrm{~h}$ and peaked at $4 \mathrm{~h}$ post-exposure to silica (Figure 4A). These findings indicate that stress-response genes are rapidly activated by crystalline silica but not by amorphous silica particles in human lung epithelial cells. It has previously been demonstrated that FOS expression is increased by alpha quartz, DQ12 (quartz) and coalmine dust with high quartz concentrations in a murine alveolar type II cell line (C10) [22, 48]. In addition, $A T F 3$ was significantly up-regulated by crystalline silica as early as 4 and up to $24 \mathrm{~h}$ (Figure 4B). In studies investigating gene expression induced by asbestos in human mesothelial and bronchial epithelial cells [30, 31, 42, 49], as well as by cigarette smoke extract in 3T3 fibroblasts [50] and benzo(a)pyrine diol-epoxide (BPDE) in bronchial epithelial cells [42], ATF3 expression is significantly increased. Moreover, Shukla et. al. [31], demonstrated that ATF3 expression was causally linked to the release of cytokines from human mesothelial cells exposed to asbestos fibers using siRNA approaches.

To our knowledge, up-regulation of $A T F 3$ by crystalline silica particles has not been reported previously. It is possible, based on our earlier work [31] and results below, that ATF3 may be linked to secreted-mediator release from bronchial epithelial cells. For example, IL6 and IL8, which are linked to early inflammatory response, are up-regulated at the mRNA level and released from lung epithelial cells, alveolar macrophages, and in BALF of mice exposed to crystalline silica [20, 43-46]. Our studies show that IL6 and IL8 mRNA levels are upregulated significantly $(\mathrm{p}<0.05)$ at 4 and $8 \mathrm{~h}$ by cristobalite, and $24 \mathrm{~h}$ as well for $I L 8$, (Figure 4C, D). In summary, data in Figure 4 show that stress-response and pro-inflammatory genes are up-regulated early and over time, peaking at $4 \mathrm{~h}$, in human bronchial epithelial cells by crystalline, but not amorphous silica particles. As both particle types are similar in size and chemical composition, non-toxic to, and taken up by BEAS $2 \mathrm{~B}$ cells, their differential effects on gene expression may be related to differences in crystallinity. 

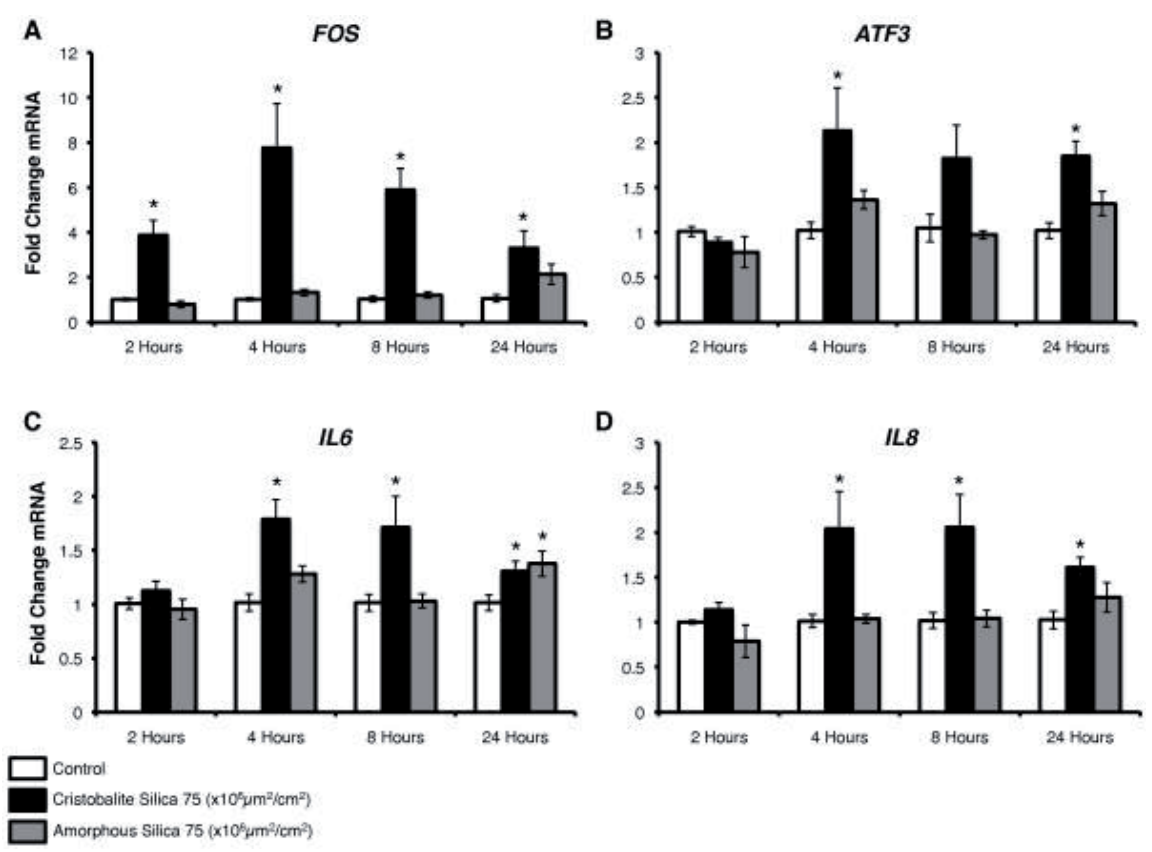

Figure 4: BEAS 2B steady state mRNA levels over time with exposure to silica particles of stressrelated and immune response genes. Time-course analysis of $m R N A$ levels by $q R T-P C R$ of $B E A S 2 B$ cells exposed to $75 \times 10^{6} \mu \mathrm{m} 2 / \mathrm{cm} 2$ cristobalite (black bars) and amorphous silica (gray bars) and unexposed controls (white bars) for 2, 4, 8 and $24 \mathrm{~h}$. Fold change in mRNA of (A) c-fos, (B) ATF3, (C) $I L-6$ and (D) IL-8. ${ }^{*} p<0.05$ compared to unexposed controls. Bars denote mean $\pm S E M$ of $N=3$ samples per group, $\dagger N=6$ samples for $c$-fos and ATF3 at $24 \mathrm{~h}$.

Cristobalite silica selectively induces pro-inflammatory and angiogenic cytokine and chemokine secretion from BEAS $2 B$ cells

Figure 5 shows the results of Bioplex assays. Levels of 9 cytokines/chemokines in medium were increased $(\mathrm{p}<0.05)$ selectively by cristobalite and 2 were decreased by both silica types when compared to unexposed control cells. As for the remainder of the 27 cytokines and chemokines surveyed, 8 (IFN $\gamma$, IP-10, IL-1 $\beta$, IL-7, IL-9, IL-10, MIP-1 $\alpha$ and MIP-1 $\beta$ ) yielded insignificant changes when compared to unexposed controls, and 8 (IL-1ra, IL-2, IL-4, IL-5, IL17, Eotaxin, GM-CSF and TNF $\alpha$ ) were below the lowest detection limit of the assay. Exposure of BEAS 2B cells to cristobalite at 75 and $150 \times 10^{6} \mu \mathrm{m}^{2} / \mathrm{cm}^{2}$ for $24 \mathrm{~h}$ caused dose-responsive and significant release of Interleukin-6 (IL-6), IL-8, IL-12 (p70), IL-13, regulated on activation normal T-cell expressed and secreted (RANTES), granulocyte colony-stimulating factor (G$\mathrm{CSF}$ ), basic fibroblast growth factor (bFGF/FGF-2), platelet-derived growth factor-BB (PDGF$\mathrm{BB}$ ), and vascular endothelial growth factor (VEGF) (Figure 5). Interestingly, amorphous silica, even at the high surface area concentration $\left(150 \times 10^{6} \mu \mathrm{m}^{2} / \mathrm{cm}^{2}\right)$ did not induce increased secretion of any of these mediators. However, amorphous silica induced a significant decrease in the amount of interleukin-15 (IL-15) detected in medium, and both cristobalite and amorphous silica caused significant decreases in the amounts of monocyte chemotactic protein-1 (MCP-1; 
MCAF) secreted by BEAS 2B cells. Possible roles of IL-12 and IL-15 have been addressed in mouse inhalation models of silicosis [51]. However, it appears that innate immunity (as opposed to adaptive responses) plays a much stronger role in the development of silicosis [52], suggesting that IL-12 may have a less likely role in silica-induced disease.

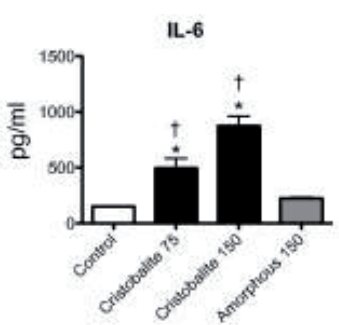

IL-15
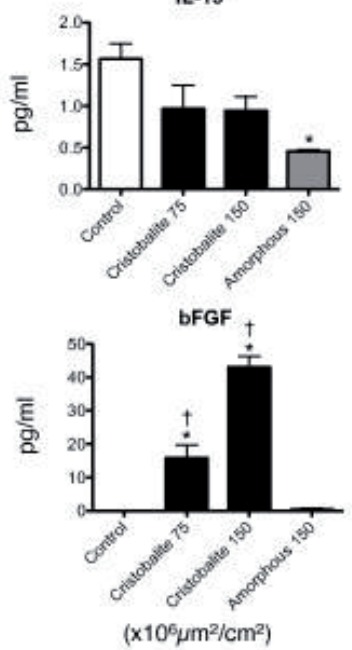

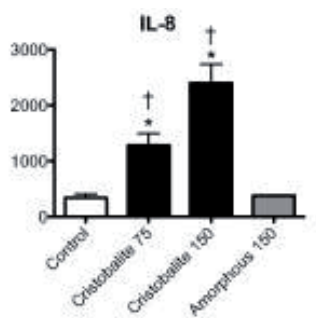

MCAF

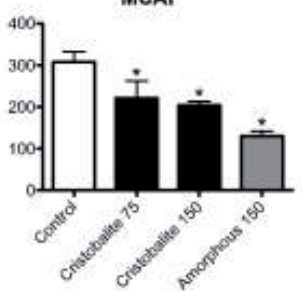

PDGF-BB

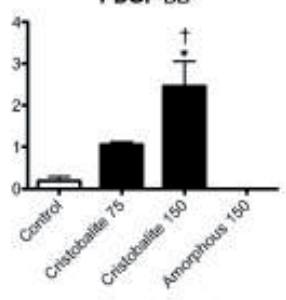

$\left(\mathrm{x} 10^{5} \mu \mathrm{m}^{2} / \mathrm{cm}^{2}\right)$

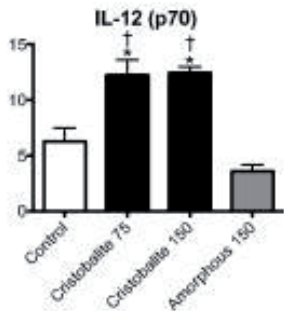

RANTES

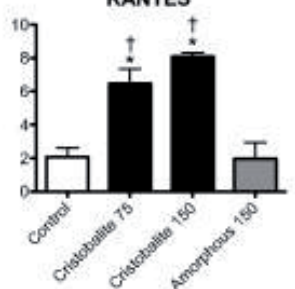

VEGF

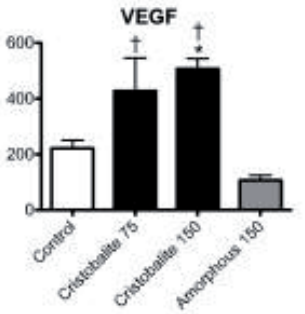

$\left(x 10^{6} \mu \mathrm{m}^{2} / \mathrm{cm}^{2}\right)$
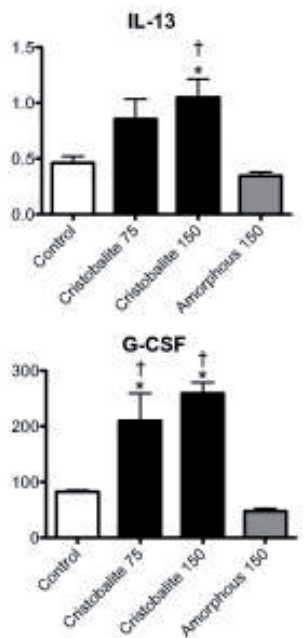

$\left(\times 10^{6} \mu \mathrm{m}^{2} / \mathrm{cm}^{2}\right)$

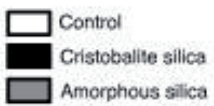

Figure 5: Bioplex analysis of secreted cytokines and chemokines in medium of BEAS $2 B$ cell exposed to silica particles $24 \mathrm{~h}$. Cell-free conditioned medium of BEAS $2 B$ exposed to silica particles for $24 \mathrm{~h}$ was assayed for 27 cytokines and chemokines. Presented in this panel are the 11 which yielded significant differences from untreated controls in levels of secreted proteins: Interleukin-6 (IL6), Interleukin-8 (IL-8), Interleukin-12 (p70) (IL-12 (p70)), Interleukin-13 (IL-13), Interleukin-15 (IL15), Monocyte chemotactic protein-1 (MCP-1 or MCAF), Regulated upon activation, normal T-cell expressed and secreted (RANTES), Granulocyte-colony stimulating factor (G-CSF), Basic fibroblast growth factor (bFGF), Platelet derived growth factor-BB polypeptide (PDGF-BB) and Vascular endothelial growth factor (VEGF). White bars (unexposed controls), black bars (cristobalite silica) and gray bars (amorphous silica) *Significantly different from unexposed controls $(p<0.05)$, $\uparrow$ Significantly different compared to amorphous silica $(p<0.05)$.

It has been shown by others that crystalline silica particles induce expression and secretion of IL-6 and IL-8 [20, 43-47], potent mediators promoting acute phase responses and inflammation in lung diseases [53-56], and thus are implicated in silicosis [57]. Increased levels of RANTES and MCP1/MCAF expression also have been reported in alveolar type II cells exposed to cristobalite silica 
[43], though increased expression of MCP-1 was seen early and had nearly diminished by 24 hours. Our studies show decreased levels of MCP-1/MCAF in medium of both crystalline and amorphous silica exposed BEAS 2B cells. Since both RANTES and MCP-1 are associated with recruitment of monocytes, lymphocytes and granulocytes [58], their decreased release by particles might indicate a mechanism of lung defense. In contrast, G-CSF is an extremely strong potentiator of neutrophilic response and recruitment (as reviewed in [59]), and is implicated in development of inflammation and pulmonary fibrosis [18].

In addition to modulators of inflammation, the proliferative and angiogenic factors, bFGF, VEGF and PDGF-BB were released from BEAS 2B cells exposed to cristobalite silica. PDGF-BB, a promoter of angiogenesis and cell proliferation, is implicated in silicosis and other pneumoconioses $[60,61]$, and its expression may correlate with disease progression. Increased levels of serum VEGF are also observed in patients with idiopathic pulmonary fibrosis (IPF) [62]. In contrast, VEGF derived from myeloid cells is anti-fibrotic in a mouse model of pulmonary fibrosis [63]. Perhaps the cell of origin or location of VEGF secretion or the concentration of VEGF plays pivotal roles in the development and progression of pulmonary fibrosis.

Our data, consistent with other studies using crystalline silica in bronchial epithelial cells, shows that epithelial cells also have the ability to secrete bFGF in response to crystalline silica $[64,65]$. However, our study shows that crystalline silica as opposed to amorphous silica particles selectively induced bFGF secretion. This may indicate an autocrine or paracrine role of epithelial cell-derived bFGF in promoting fibrotic disease as it has been shown that bFGF promotes fibroblast proliferation in a transforming growth factor- $\beta$ (TGF- $\beta$ ) model of epithelialmesenchymal transition $[66,67]$ and in the development and progression of interstitial pulmonary fibrosis (IPF) [68]. Additionally, it has been shown that bFGF is associated with mast cells in human silicotic lungs [69].

Taken together, a number of pro-inflammatory, proliferative and angiogenic factors are released from lung epithelial cells, which are initial target cells of silica-induced disease. How these factors may interact with and regulate the airway environment is crucial in delineation of the role the epithelium plays in initiation and progression of silica-induced fibrosis.

\section{Assessment of cell viability and gene expression induced by silica particles in primary human bronchial epithelial cells (NHBE)}

NHBE cells were used to confirm results from experiments with BEAS 2B cells as these cells are primary as opposed to immortalized human bronchial epithelial cells and may be more responsive to particles. Figure 6 shows changes in cell viability and gene expression in primary NHBE cells by cristobalite and amorphous silica particles. When compared to BEAS 2B cells, NHBE cells were more sensitive to the toxicity of silica particles. After $24 \mathrm{~h}$, both types of silica particles at 15,75 and $150 \times 10^{6} \mu \mathrm{m} 2 / \mathrm{cm} 2$ caused between $25-46 \%$ loss of viable cells (Figure 6A). Although particle exposures induced significant $(p<0.05)$ decreases in the percentage of viable cells compared to unexposed controls, no significant differences were observed between toxicity of cristobalite vs. amorphous silica at equal surface area concentrations. Based on these results, we chose to expose NHBE cells to cristobalite silica at the lowest (15) and a higher (75) surface area concentration and amorphous silica at $75 \times 10^{6} \mu \mathrm{m} 2 / \mathrm{cm} 2$ in microarray analyses. Cristobalite at $15 \times 10^{6} \mu \mathrm{m} 2 / \mathrm{cm} 2$ induced only 6 significant gene changes, though at the higher 
dose (75 X $\left.10^{6} \mu \mathrm{m} 2 / \mathrm{cm} 2\right), 339$ significant changes were detected. Interestingly, this is nearly the same number of significant changes (333) induced in BEAS 2B cells at the same exposure concentration (Figure 3A). In addition, amorphous silica at $75 \times 10^{6} \mu \mathrm{m} 2 / \mathrm{cm} 2$, i.e. half the dose used in BEAS 2B cells (Figure 3A), produced 42 significant changes.

Gene ontology analysis of the gene expression alterations in NHBE cells exposed to silica particles was performed to compare the trends seen in BEAS $2 \mathrm{~B}$ cells and to allow examination of similarities and differences between cells (Figure 6 C-E, Supplementary figure 2). The gene expression pattern of NHBE cells exposed to cristobalite or amorphous silica particles followed nearly the same patterns of expression with most gene changes categorized in the realms of cell signaling and proliferation, apoptosis, protein metabolic process and immune response.
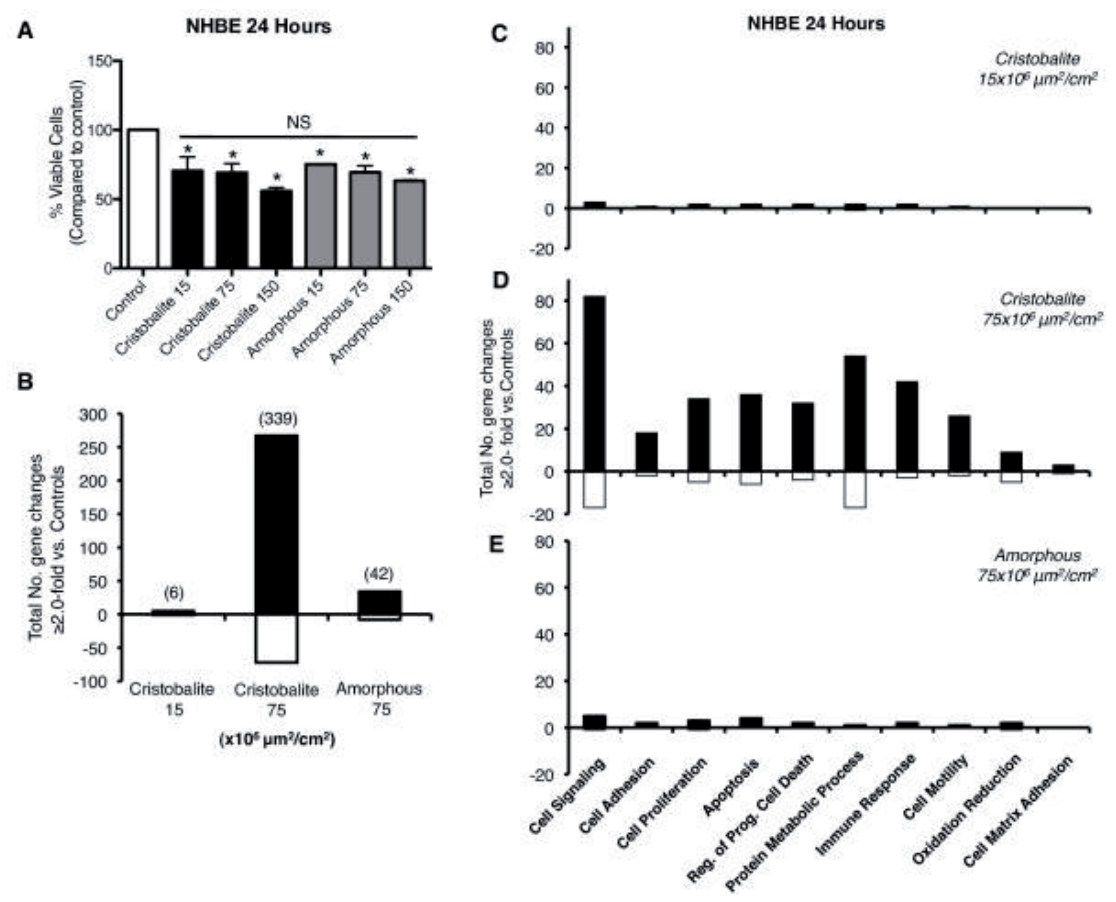

Figure 6: Assessment of cell viability and microarray analysis of $\mathrm{NHBE}$ cells in response to silica particle exposure. Effects of silica particles on NHBE cells after 24 h exposures. (A) Cell viability assessed by the Trypan blue exclusion assay ( $N=3$ in 2 independent experiements) $* p<0.05$ compared to controls. NS = Not Significant for comparison of all exposure groups. (B) Total number of gene changes $\geq 2$.0-fold vs. Controls induced by silica particle exposure for $24 \mathrm{~h}$ (total number gene changes). (C-E) Gene ontology analysis of total number of gene changes in 10 categories of interest (Cell Signaling, Cell Adhesion, Cell Proliferation, Apoptosis, Regulation of Programmed Cell Death, Protein Metabolic Process, Immune Response, Cell Motility, Oxidation-Reduction and Cell Matrix Adhesion) for (A-D) positive values (number of genes up-regulated) and negative values (number of gene down-regulated). 
Table 3 presents the top 10 genes up and down-regulated by cristobalite and amorphous silica particles in NHBE cells. IL8, at the top of this list, was up-regulated by cristobalite 4.37 and 28.47 -fold at 15 and $75 \times 10^{6} \mu \mathrm{m} 2 / \mathrm{cm} 2$ respectively. However, there was no increase in expression by amorphous silica particles. Interestingly, TXNIP was up-regulated by both cristobalite and amorphous silica by 9.74 -fold and 31.14-fold respectively. This protein binds to NLRP3 and promotes inflammasome activation in response to oxidative stress [70]. Other genes of interest in this table include BIRC3, BCL2A1 and TNFAIP3, which are involved in regulation of apoptosis. When comparing the top 10 genes altered in expression by crystalline silica particles in BEAS 2B and NHBE cells, only one gene, Solute carrier family 38, member 4 (SLC38A4), a sodium-dependent amino acid symporter, is common to both cells (within the top 10). However, when all gene expression changes by cristobalite silica were compared in BEAS 2B and NHBE cells (Table 4), we found 60 genes commonly altered (57 up-regulated and 3 down-regulated). Of these 60 genes, 36 were altered in both cell types at the same surface area concentration $\left(75 \times 10^{6}\right.$ $\mu \mathrm{m} 2 / \mathrm{cm} 2)$ and 57 were commonly altered in NHBE cells at $75 \times 10^{6} \mu \mathrm{m} 2 / \mathrm{cm} 2$ vs. BEAS $2 B$ at $150 \times 10^{6} \mu \mathrm{m} 2 / \mathrm{cm} 2$. 
Table 3: Top 10 genes affected by 24 h exposure to silica particles in NHBE. Top 10 genes in NHBE cells up and down-regulated by $24 \mathrm{~h}$ exposure to cristobalite silica at 15 and $775 \times 10^{6} \mu^{2} / \mathrm{cm}^{2}$ $\mu^{2} / \mathrm{cm}^{2}$ and amorphous silica particles at $75 \times 10^{6} \mathrm{\mu m}^{2} / \mathrm{cm}^{2}$.

\begin{tabular}{|c|c|c|c|}
\hline \multirow[t]{3}{*}{ Gene Name (Abbreviation) } & \multicolumn{3}{|c|}{ Fold Change $^{\text {a }}$} \\
\hline & \multicolumn{2}{|c|}{ Cristobalite } & \multirow{2}{*}{$\begin{array}{c}\text { Amorphous } \\
\mathbf{7 5}^{\mathrm{b}}\end{array}$} \\
\hline & $15^{\mathrm{b}}$ & $7^{\mathrm{b}}$ & \\
\hline \multicolumn{4}{|l|}{ Up-regulated $\Delta$} \\
\hline Interleukin 8 (IL8) & 4.37 & 28.47 & $\mathrm{NC}$ \\
\hline Baculoviral IAP repeat-containing 3 (BIRC3) & 2.02 & 19.81 & $\mathrm{NC}$ \\
\hline BCL2-related protein A1 (BCL2A1) & $\mathrm{NC}$ & 19.27 & $\mathrm{NC}$ \\
\hline Tumor necrosis factor, alpha-induced protein 3 (TNFAIP3) & 2.33 & 17.11 & $\mathrm{NC}$ \\
\hline Interleukin 1 family, member 9 (IL1F9) & $\mathrm{NC}$ & 14.14 & $\mathrm{NC}$ \\
\hline GTP binding protein over expressed in skeletal muscle (GEM) & $\mathrm{NC}$ & 12.92 & $\mathrm{NC}$ \\
\hline CD83 molecule (CD83) & $\mathrm{NC}$ & 11.47 & $\mathrm{NC}$ \\
\hline $\begin{array}{l}\text { Transcribed locus, strongly similar to NP_006463.2 thioredoxin } \\
\text { interacting protein }\end{array}$ & $\mathrm{NC}$ & 11.09 & 27.95 \\
\hline Inhibin, beta A (INHBA) & $\mathrm{NC}$ & 10.25 & $\mathrm{NC}$ \\
\hline Thioredoxin interacting protein (TXNIP) & $\mathrm{NC}$ & 9.74 & 31.14 \\
\hline Heat shock 70kDa protein 1A (HSPA1A) & 2.01 & 7.56 & 2.57 \\
\hline Crystallin, mu (CRYM) & $\mathrm{NC}$ & 4.97 & 2.35 \\
\hline G0/G1 switch 2 (G0S2) & $\mathrm{NC}$ & 3.51 & 3.12 \\
\hline Transcribed locus & $\mathrm{NC}$ & $\mathrm{NC}$ & 2.73 \\
\hline Calcium binding tyrosine-(Y)-phosphorylation regulated (CABYR) & $\mathrm{NC}$ & 2.51 & 2.55 \\
\hline Non-protein coding RNA 84 (NCRNA00084) & $\mathrm{NC}$ & $\mathrm{NC}$ & 2.53 \\
\hline $\begin{array}{l}\text { Sarcoglycan, gamma (35kDa dystrophin-associated glycoprotein) } \\
\text { (SGCG) }\end{array}$ & $\mathrm{NC}$ & $\mathrm{NC}$ & 2.50 \\
\hline Cytoplasmic FMR1 interacting protein 2 (CYFIP2) & $\mathrm{NC}$ & $\mathrm{NC}$ & 2.48 \\
\hline \multicolumn{4}{|l|}{ Down-regulated $\nabla$} \\
\hline Sulfatase & $\mathrm{NC}$ & 3.59 & $\mathrm{NC}$ \\
\hline $\begin{array}{l}\text { Peroxisome proliferator-activated receptor gamma, coactivator } 1 \\
\text { alpha (PPARGC1A) }\end{array}$ & $\mathrm{NC}$ & 3.52 & $\mathrm{NC}$ \\
\hline Solute carrier family 38, member 4 (SLC38A4) & $\mathrm{NC}$ & 3.43 & $\mathrm{NC}$ \\
\hline $\begin{array}{l}\text { Amyotrophic lateral sclerosis } 2 \text { (juvenile) chromosome region, } \\
\text { candidate } 8 \text { (ALS2CR } 8 \text { ) }\end{array}$ & $\mathrm{NC}$ & 3.18 & 2.00 \\
\hline Growth arrest-specific 1 (GAS1) & $\mathrm{NC}$ & 2.84 & $\mathrm{NC}$ \\
\hline Hexokinase 2 (HK2) & $\mathrm{NC}$ & 2.69 & $\mathrm{NC}$ \\
\hline F-box protein 9 (FBXO9) & $\mathrm{NC}$ & 2.68 & $\mathrm{NC}$ \\
\hline Hairy/enhancer-of-split related with YRPW motif 1 (HEY1) & $\mathrm{NC}$ & 2.62 & $\mathrm{NC}$ \\
\hline CDNA FLJ37852 fis, clone BRSSN2014513 & $\mathrm{NC}$ & 2.61 & $\mathrm{NC}$ \\
\hline Growth hormone receptor (GHR) & $\mathrm{NC}$ & 2.59 & $\mathrm{NC}$ \\
\hline $\begin{array}{l}\text { Solute carrier family 16, member } 7 \text { (monocarboxylic acid transporter } \\
\text { 2) (SLC16A7) }\end{array}$ & $\mathrm{NC}$ & 2.50 & $\mathrm{NC}$ \\
\hline $\begin{array}{l}\text { Transglutaminase } 3 \text { (E polypeptide, protein-glutamine-gamma- } \\
\text { glutamyltransferase) (TGM3) }\end{array}$ & 2.13 & $\mathrm{NC}$ & $\mathrm{NC}$ \\
\hline Follistatin (FST) & $\mathrm{NC}$ & $\mathrm{NC}$ & 2.43 \\
\hline Homo sapiens clone FLB9440 PRO2550 mRNA, complete cds & $\mathrm{NC}$ & 2.32 & 2.18 \\
\hline
\end{tabular}


Ribonucleotide reductase M2 polypeptide (RRM2)

$\begin{array}{lll}\mathrm{NC} & \mathrm{NC} & 2.12 \\ \mathrm{NC} & 2.23 & 2.06\end{array}$

PPPDE peptidase domain containing 1 (PPPDE1)

$\mathrm{NC} \quad 2.23$

2.06

${ }^{a}$ Fold change (mRNA) expression $(p<0.05)$ cut-off $\geq 2.0$-fold compared to controls $(n=3)$. Top 10 genes from each exposure group.

${ }^{\text {b }}$ Silica particle concentrations: ( X $10^{6} \mu \mathrm{m} 2 / \mathrm{cm} 2$ )

$\mathrm{NC}=$ No Change

Table 4: Genes commonly affected by 24 h cristobalite exposure in NHBE and BEAS $2 B$ cell. Sixty genes (57 up-regulated and 3 down-regulated) found commonly altered by exposure to cristobalite silica in NHBE cells and BEAS $2 B$ cells from microarray analysis.

\begin{tabular}{|c|c|c|c|}
\hline \multirow{2}{*}{$\begin{array}{l}\text { Gene Name (Abbreviation) } \\
\text { Cell Type } \\
\text { (Cristobalite) }^{\mathrm{b}}\end{array}$} & \multicolumn{3}{|c|}{ Fold Change $^{\mathrm{a}}$} \\
\hline & $\begin{array}{l}\text { NHBE } \\
(75)\end{array}$ & $\begin{array}{l}\text { BEAS 2B } \\
\quad(75)\end{array}$ & $\begin{array}{l}\text { BEAS 2B } \\
\text { (150) }\end{array}$ \\
\hline \multicolumn{4}{|l|}{ Up-regulated $\Delta$} \\
\hline Interleukin 8 (IL8) & 28.47 & 2.12 & 2.65 \\
\hline BCL2-related protein A1 (BCL2A1) & 19.27 & $\mathrm{NC}$ & 3.08 \\
\hline Tumor necrosis factor, alpha-induced protein 3 (TNFAIP3) & 17.11 & 2.23 & 2.95 \\
\hline GTP binding protein overexpressed in skeletal muscle (GEM) & 12.92 & $\mathrm{NC}$ & 2.13 \\
\hline Inhibin, Beta A (INHBA) & 10.25 & 2.24 & 3.62 \\
\hline Interleukin 24 (IL24) & 7.62 & 3.88 & 8.54 \\
\hline Heat shock $70 \mathrm{kDa}$ protein $1 \mathrm{~A}$ (HSPA1A) & 7.56 & $\mathrm{NC}$ & 2.34 \\
\hline Chemokine (C-X-C motif) ligand 3 (CXCL3) & 7.42 & $\mathrm{NC}$ & 2.42 \\
\hline TNF receptor-associated factor 1 (TRAF1) & 7.30 & 2.86 & 4.22 \\
\hline Radical S-adenosyl methionine domain containing 2 (RSAD2) & 6.92 & 5.45 & 23.25 \\
\hline Early growth response 1 (EGR1) & 6.72 & 2.65 & 4.63 \\
\hline Coiled-coil domain containing 85B (CCDC85B) & 6.46 & 3.44 & 6.11 \\
\hline FOS-like antigen 1 (FOSL1) & 5.80 & 2.47 & 3.18 \\
\hline Early growth response 3 (EGR3) & 5.31 & $\mathrm{NC}$ & 2.65 \\
\hline Jun oncogene (JUN) & 4.99 & $\mathrm{NC}$ & 2.00 \\
\hline Matrix metallopeptidase 1, interstitial collagenase (MMP1) & 4.99 & 16.12 & 44.68 \\
\hline 2'-5'-oligoadenylate synthetase-like (OASL) & 6.80 & $\mathrm{NC}$ & 18.85 \\
\hline cAMP responsive element binding protein 5 (CREB5) & 3.87 & 3.25 & 4.10 \\
\hline $\begin{array}{l}\text { Transcribed locus, strongly similar to NP_000337.1 transcription } \\
\text { factor SOX9 (SOX9) }\end{array}$ & 3.84 & $\mathrm{NC}$ & 2.12 \\
\hline Interferon induced with helicase $\mathrm{C}$ domain 1(IFIH1) & 3.79 & $\mathrm{NC}$ & 3.86 \\
\hline Heat shock 70kDa protein 6 (HSP70B') & 3.70 & 4.08 & 14.39 \\
\hline Kynureninase (L-kynurenine hydrolase) (KYNU) & 3.66 & $\mathrm{NC}$ & 2.19 \\
\hline Prostaglandin-endoperoxide synthase 2 (PTGS2) & 3.56 & $\mathrm{NC}$ & 2.54 \\
\hline Superoxide dismutase 2, mitochondrial (SOD2) & 3.53 & $\mathrm{NC}$ & 2.86 \\
\hline Interleukin 1 receptor-like 1 (IL1RL1) & 3.52 & 2.89 & 5.36 \\
\hline Interferon-induced protein with tetratricopeptide repeats 1 (IFIT1) & 3.45 & 6.01 & 16.26 \\
\hline Interferon-induced protein 44 (IFI44) & 3.36 & 7.51 & 19.90 \\
\hline Nuclear receptor subfamily 1 , group D, member 1 (NR1D1) & 3.24 & 3.01 & 4.72 \\
\hline Interferon stimulated exonuclease gene $20 \mathrm{kDa}$ (ISG20) & 3.21 & $\mathrm{NC}$ & 2.48 \\
\hline Plasminogen activator, urokinase receptor (PLAUR) & 3.14 & $\mathrm{NC}$ & 2.35 \\
\hline
\end{tabular}


Transmembrane protein 40 (TMEM40)

Distal-less homeobox 2 (DLX2)

$3.06 \quad \mathrm{NC} \quad 4.15$

Niacin receptor 2 (NIACR2 OR GPR109B)

$\begin{array}{lll}2.99 & 2.34 & 3.78\end{array}$

Human 28S ribosomal RNA gene, complete cds. (RNA28S1)

$2.87 \quad 3.14$

$\mathrm{NC}$

Histone deacetylase 9 (HDAC9)

$2.86 \quad \mathrm{NC} \quad 2.21$

2'-5'-oligoadenylate synthetase 1, 40/46kDa (OAS1)

$2.84 \quad 3.24 \quad \mathrm{NC}$

Nuclear receptor subfamily 4 , group A, member 3 (NR4A3)

$7.09 \quad \mathrm{NC} \quad 20.53$

Oxidative stress induced growth inhibitor 1 (OSGIN1)

$\begin{array}{lll}2.79 & 2.58 & 3.49\end{array}$

Smoothelin (SMTN)

$2.79 \quad \mathrm{NC} \quad 2.48$

Heme oxygenase (decycling) 1 (HMOX1)

Basic helix-loop-helix family, member e41 (BHLHE41)

ADAM metallopeptidase domain 8 (ADAM8)

Crystallin, alpha B (CRYAB)

$\begin{array}{lll}2.74 & 2.38 & 2.72\end{array}$

$2.69 \quad \mathrm{NC} \quad 2.57$

$2.64 \quad \mathrm{NC} \quad 2.34$

$2.62 \quad \mathrm{NC} \quad 2.08$

Colony stimulating factor 2 (granulocyte-macrophage) (CSF2)

$\begin{array}{lll}2.58 & 2.26 \quad 2.67\end{array}$

$\begin{array}{lll}2.53 & 4.20 \quad 7.37\end{array}$

Calcium binding tyrosine-(Y)-phosphorylation regulated (CABYR) $\quad 2.51 \quad$ NC $\quad 2.19$

Chromosome 1 open reading frame 38 (C1orf38)

2'-5'-oligoadenylate synthetase 3, 100kDa (OAS3)

$2.44 \quad \mathrm{NC} \quad 3.04$

$\begin{array}{lll}2.29 & 2.41 \quad 3.84\end{array}$

Sprouty homolog 4 (Drosophila) (SPRY4)

$\begin{array}{lll}2.26 & 2.98 & 4.64\end{array}$

Dual specificity phosphatase 5 (DUSP5)

Ring finger protein 39 (RNF39)

2.23

Leukemia inhibitor factor (Cholinergic differentiation factor) (LIF)

2.21

Sphingosine kinase 1 (SPHK1)

$2.11 \quad 3.20$

4.88

2.10

2.12

2.59

Interleukin 1, alpha (IL1A)

2.09

3.97

6.17

FBJ murine osteosarcoma viral oncogene homolog B (FOSB)

2.07

3.65

6.37

Serpin peptidase inhibitor, clade B (ovalbumin), member 2 (PAI2)

2.04

2.16

4.33

DEAD (Asp-Glu-Ala-Asp) box polypeptide 60 (DDX60)

\section{Down-regulated}

Sulfatase 1 (SULF1)

2.02

2.26

5.01

Peroxisome proliferator-activated receptor gamma, coactivator 1

alpha (PPARGC1A)

$\begin{array}{lll}3.52 & 2.85 & 3.75\end{array}$

Chromosome 5 open reading frame 13 (C5orf13)

$2.05 \quad \mathrm{NC}$

2.21

\footnotetext{
${ }^{a}$ Fold change (mRNA) expression $(p<0.05)$ cut-off $\geq 2.0$-fold compared to controls $(n=3)(60$ genes total)

${ }^{\text {b }}$ Cristobalite silica particle concentrations: ( X $10^{6} \mu \mathrm{m} 2 / \mathrm{cm} 2$ )

$\mathrm{NC}=$ No Change; (Listed in descending order of fold-change in NHBE).
}

Within this list of commonly affected genes there are a number of transcription factors, cytokines/chemokines and receptors, oxidative stress-related, and genes related to the regulation of apoptosis, proliferation, cell signaling and ECM breakdown. The transcription factors, FOSL1 (fra-1), FOSB and, JUN, members of the AP-1 complex family, as well as CREB5, which can also interact with jun family members, were up-regulated by cristobalite in both cell lines. The AP-1 trancription factor complex appears to play a major role in cellular response to crystalline silica [48]. In addition, the transcriptional regulators EGR1 and EGR3 were induced by cristobalite silica. 
A number of pro-inflammatory mediators were also increased at the mRNA level by cristobalite in NHBE and BEAS 2B cells including, IL-1 $, I L-6, I L-8, I L 24, C X C L 3$, (MIP-2 $\alpha$ ), CSF2 (GM$\mathrm{CSF})$ and the IL1RL1. Some of these genes (IL-1 $\beta, I L 1 R L 1)$ and their proteins are involved in regulation and activity of the NALP3 inflammasome, a signaling complex that responds to "danger signals" and promotes an inflammatory response after addition of silica to human THP-1 macrophages [71]. In line with the hypothesis that oxidative stress mediates inflammasome activity by silica, oxidative-stress response-genes, PTGS2 (COX2), SOD2 (MnSOD), HMOX-1 and OSGIN1 were also up-regulated by cristobalite in NHBE and BEAS 2B cells. These findings also reinforce the observations that inflammation and oxidative stress are two of the driving forces in silica-induced disease mechanisms [72].

A number of other genes were also altered in expression by cristobalite in both cell lines, including genes involved in ECM breakdown (MMP1 and PLAUR), genes involved in cell signaling regulation (HDAC9), and regulators of MAPK signaling (SPRY4 and DUSP5). Other genes are involved in anti-viral activity and interferon-response including, RSAD2, OAS1, OAS3, OASL, IFIH1, IFIT2, IFI44 and ISG20. Of those genes, IFIT1 and IFI44 were up-regulated in both cell types by amorphous silica particles. 


\section{CONCLUSIONS}

Particulates, especially in the realm of nanotoxicology, are rapidly becoming a question of concern regarding health effects in occupational setting as well as in the environment. Our studies and a recent report in the literature [73] demonstrate that gene expression profiling in human lung epithelial cells in vitro can be used to determine the relative pathogenicity of potentially harmful particulates (i.e. crystalline silica and airborne particulate matter, respectively) as opposed to viability studies and metabolic testing. Changes in expression of genes related to inflammation, oxidative stress, and proliferation as well as the secretion of proinflammatory, angiogenic and proliferative cytokines and chemokines re-emphasize the complex reactions of the lung epithelium to crystalline silica particles and their relation to development of fibrosis [11].

Our studies compared two particles of the same chemical make up $\left(\mathrm{SiO}_{2}\right)$, but of different habit (i.e. crystalline vs. amorphous). The responses observed suggest that the crystallinity of cristobalite may dictate early molecular responses as well as dissolution of particles over time in the lung. Moreover, we show that the magnitude and patterns of differential gene expression by crystalline silica are similar in both BEAS 2B and NHBE cells, suggesting that immortalized and established epithelial cell lines can be used as in vitro models to predict the pathogenicity of potentially harmful respirable particulates as opposed to expensive and timeconsuming isolation of human primary cells and animal inhalation studies. 


\section{METHODS}

\section{Human bronchial epithelial cell cultures}

Non-tumorigenic human bronchial epithelial cells (Ad12-SV40 immortalized) BEAS 2B (ATCC, Manassas, VA) were grown and maintained in DMEM/F12 50:50 media containing $10 \%$ Fetal Bovine Serum (FBS) (CellGro ${ }^{\circledR}$ Mediatech inc, Manassas, VA), with penicillin (50 units $/ \mathrm{ml})$, streptomycin $(100 \mu \mathrm{g} / \mathrm{ml})$ (Invitrogen, Carlsbad, CA), hydrocortisone (100 $\mu \mathrm{g} / \mathrm{ml})$, insulin $(2.5 \mu \mathrm{g} / \mathrm{ml})$, transferrin $(2.5 \mu \mathrm{g} / \mathrm{ml})$ and selenium $(2.5 \mu \mathrm{g} / \mathrm{ml})$ (Sigma, St. Louis, MO). BEAS 2B cells culture flasks and plates (BD, Franklin Lakes, NJ) were precoated with a mixture of fibronectin (Sigma, St. Louis, MO) $(0.01 \mathrm{mg} / \mathrm{ml})$, bovine collagen type I $(0.03 \mathrm{mg} / \mathrm{ml})$ (Invitrogen, Carlsbad, CA) and bovine serum albumin $(0.01 \mathrm{mg} / \mathrm{ml})$ (Sigma, St. Louis, MO), dissolved in DMEM/F12 media for 24 hours at $37^{\circ} \mathrm{C}$ prior to culturing cells. Prior to exposures, medium was aspirated and replaced with a reduction medium containing $0.5 \%$ FBS. Primary normal human bronchial epithelial cells (NHBE17917) were purchased from Lonza, Clonetics ${ }^{\circledR}$. NHBE cells were cultured and maintained in BEGM ${ }^{\circledR}$ and with Reagentpack ${ }^{\mathrm{TM}}$ subculture reagents (Trypsin/EDTA, Trypsin Neutralizing Solution and Hepes-buffered saline solution), all purchased from Lonza, Clonetics ${ }^{\circledR}$. (Switzerland) were used for subculture following manufacturers protocol.

\section{Synthesis, characterization, and determination of surface areas of silica particles.}

The amorphous silica microparticles were prepared according to the procedure of De et al [74] in the Department of Chemistry, University of Vermont, Burlington, VT, USA. Briefly, tetraethylorthosilicate $(32.0 \mathrm{~g}, 0.143 \mathrm{~mol})$ was combined with water $(11.0 \mathrm{~g}, 0.611 \mathrm{~mol})$ and stirred rapidly $(600 \mathrm{rpm})$ to form an emulsion. To this emulsion was rapidly added glacial acetic acid ( $36.8 \mathrm{~g}, 0.643 \mathrm{~mol})$, and stirring was continued for $60 \mathrm{~s}$, at which point stirring was terminated. The mixture was then allowed to sit quiescently for $45 \mathrm{~min}$. Afterward the particles were filtered and washed extensively with water $(5 \mathrm{~mL} \times 4)$ and ethanol $(5 \mathrm{~mL} \times 3)$. After air drying for several hours, the particles were dried overnight under vacuum. Finally, the particles were ground with a mortar and pestle to yield a freely-flowing, white powder ( $4.8 \mathrm{~g}, 56 \%$ yield). Samples were mounted for SEM by dusting onto carbon tape applied to aluminum sample stubs. They were then sputter coated with $\mathrm{Pd} / \mathrm{Au}$ for 3.5 minutes in a Polaron sputter coater (Model 5100). Specimens were then examined with a JSM 6060 scanning electron microscope (JEOL USA, Inc., Peabody, MA) operating at an accelerating voltage of $25 \mathrm{kV}$ and a working distance of $10 \mathrm{~mm}$. Size distribution was determined by measuring the diameter of at least 200 particles. Cristobalite silica particles were characterized previously [75]. Surface area of particles was determined by BET nitrogen adsorption analysis. Nitrogen adsorption and desorption isotherms were obtained on a Micromeritics TriStar instrument after samples were degassed overnight under vacuum. Surface areas were measured using the BET method, and pore size distributions were calculated from a modified KJS method using the adsorption branch [76-78]. 


\section{Particle exposure to cells}

Prior to exposure, particles were weighed in scintillation vials and placed under UV light overnight to be sterilized. Particles were then submerged in Hanks' Balanced Salt Solution (HBSS) (CellGro ${ }^{\circledR}$ Mediatech inc, Manassas, VA) at $[1.0-2.0 \mathrm{mg} / \mathrm{ml}]$. Particle suspensions were sonicated in a waterbath sonicator for 15 minutes followed by being trichurated 5 times through a 22-gauge needle. Suspensions were then administered to cell culture plates and briefly shaken to assure dispersion of particles before they settle.

\section{Assessment of cell viability}

After $24 \mathrm{~h}$, cells were collected by detatching with $0.25 \%$ Trypsin (Invitrogen, Carlsbad, CA) solution in HBSS, and a final volume of $1.5 \mathrm{ml}(0.5 \mathrm{ml}$ trypsin solution $+1.0 \mathrm{ml} 10 \%$ FBS medium was collected on ice. This solution was diluted $1: 5$ in a solution of $0.4 \%$ trypan blue (MP Biomedicals, Solon, OH), which is a dye retained by dead cells and excluded by viable cells and HBSS. After $5 \mathrm{~min}$, unstained viable cells were counted using a hemocytometer to determine the total number of viable cells per dish and particle exposed groups were compared unexposed controls as described previously [79] For each group (particle type/concentration) $\mathrm{n}=3$ and experiments were performed in triplicate (BEAS 2B) and duplicate (NHBE).

\section{Scanning electron microscopy (SEM)}

For imaging of cristobalite silica and amorphous silica particles, 0.0029 or $0.0026 \mathrm{~g}$ was diluted to a final concentration of 1.45 and $1.3 \mu \mathrm{g} / \mathrm{ml}$ ( $4.0 \mathrm{ml}$ total volume), respectively, in a solution of $6 \%$ ethanol and $\mathrm{ddH} 2 \mathrm{O}$ by serial dilution. Silica particle dilutions were filtered through a $0.4 \mu \mathrm{m}$ Nucleopore Track-Etch membrane (Fisher Scientific, Pittsburgh, PA) followed by a rinse with $1.0 \mathrm{ml} 100 \%$ ethanol and drying overnight. Half of the dried filter was adhered to a standard aluminum specimen stub with colloidal silver paste (Electron Microscope Sciences, Hatfield, PA) followed by sputter coating with gold and palladium using a Polaron sputter coater (Model 5100; Quorum Technologies, East Sussex, UK) Specimens were imaged and EDS spectra taken on and off particles. BEAS 2B cells were grown on Thermonox plastic cover slips (Nalge Nunc International, Naperville, IL). Prior to seeding cells, cover slips were coated with fibronectin $(0.01 \mathrm{mg} / \mathrm{ml})$, bovine collagen type I $(0.03 \mathrm{mg} / \mathrm{ml})$ and bovine serum albumin $(0.01 \mathrm{mg} / \mathrm{ml})$ dissolved in DMEM/F12 medium for $24 \mathrm{~h}$ at $37^{\circ} \mathrm{C}$. Cells were grown to near confluency and then put in reduction medium $(0.5 \%$ FBS) $24 \mathrm{~h}$ prior to incubation with particles. Cells were exposed to silica particles for 2 and $24 \mathrm{~h}$ before fixation. Cells were fixed with $2 \%$ glutaraldehyde in $0.1 \mathrm{M}$ phosphate buffered saline (PBS) for $1 \mathrm{~h}$ at $4^{\circ} \mathrm{C}$. Samples then rinsed for $5 \mathrm{~min}$ in PBS (3X) and then (1X) 5 minutes in $0.05 \mathrm{M}$ cacodylate buffer $(\mathrm{pH} 7.2)$. Samples then post-fixed with $1 \%$ osmium tetroxide in $0.05 \mathrm{M}$ cacodylate buffer $(\mathrm{pH} 7.2)$ for $1 \mathrm{~h}$ at room temperature, then rinsed (3X) for $5 \mathrm{~min}$ in $0.05 \mathrm{M}$ cacodylate buffer. Samples immersed in $1 \%$ tannic acid in $0.05 \mathrm{M}$ cacodylate buffer for $1 \mathrm{~h}$ at room temperature and in the dark (covered with foil), rinsed $1 \mathrm{X}$ for $5 \mathrm{~min}$ in cacodylate buffer and $3 \mathrm{X}$ for $5 \mathrm{~min}$ in distilled water. Samples incubated with $0.5 \%$ uranyl acetate in distilled water for $1 \mathrm{hr}$ at room temperature in the dark (covered with 
foil), rinsed $3 \mathrm{X}$ for $5 \mathrm{~min}$ in distilled water, and stored in $0.05 \mathrm{M}$ cacodylate buffer at $4^{\circ} \mathrm{C}$. Cover slips were put in cylindrical holder and dehydrated in ethanol series: using $10 \mathrm{~min}$ changes in $35,50,70,85 \%$ up to $95 \%$ ethanol, then 220 min changes in $95 \%$ ethanol, followed by three 15 min changes in anhydrous $100 \%$ ethanol. Samples were then criticalpoint dried and fixed to aluminium specimen mounts with carbon paint and dried overnight in a desiccator. Samples were then sputter-coated with gold and palladium in a Polaron sputter coater (Model 5100) and imaged on JSM-6060 scanning electron microscope (JEOL USA, Inc. Peabody, MA).

\section{RNA isolation and microarray profiling}

Total RNA was prepared using an RNeasy ${ }^{\circledR}$ Plus Mini Kit according to the manufacturers' protocol (Qiagen, Valencia, CA), as published previously [80]. All procedures were performed by the Microarray analysis core facility of the Vermont Genetics Network (VGN) using a standard Affymetrix protocol as described previously [80, 81]. GeneChip ${ }^{\circledR}$ Human Genome U133A 2.0 arrays (Affymetrix, Santa Clara, CA) targeting 18,400 human transcripts were scanned twice (Hewlett-Packard GeneArray Scanner), the images overlaid, and the average intensities of each probe cell compiled. Microarray data were analyzed using GeneSifter software (VizX Labs, Seattle, WA). This program used a t-test for pair- wise comparison and a Benjamini-Hochberg test for false discovery rate (FDR 5\%) to adjust for multiple comparisons. A 2-fold cutoff limit was used for analysis (only genes up or downregulated $\geq 2$.0-fold vs. unexposed controls)

\section{Quantitative real-time polymerase chain reaction (qRT-PCR)}

Total RNA $(1 \mu \mathrm{g})$ was reverse-transcribed with random primers using the AMV Reverse Transcriptase kit (Pro-mega, Madison, WI) according to the recommendations of the manufacturer, as described previously [80]. To quantify gene expression, the cDNA was amplified by TaqMan ${ }^{\circledR}$ qRT-PCR using the 7700 Sequence Prism Detector (Perkin Elmer Applied Biosystems, Foster City, CA). Fold change in the genes of interest was calculated using the $\Delta \Delta \mathrm{Ct}$ method. Duplicate assays were performed with all samples. The values obtained from cDNAs and hypoxanthine phosphoribosyl transferase (hprt) controls provided relative gene expression levels for the gene locus investigated. The Assays-On-Demand ${ }^{\mathrm{TM}}$ primer and probe sets used for all qRT-PCR experiments were purchased from Applied Biosystems (Foster City, CA).

\section{Bioplex analysis of cytokines and chemokines in medium of BEAS 2B cells}

To quantify cytokine and chemokine levels in conditioned medium of BEAS 2B cells exposed to silica particles, a multiplex suspension protein array was performed using the Bioplex protein array system and a human cytokine 27-plex panel (Bio-Rad, Hercules, CA), as previously described [29]. This method of analysis is based on Luminex technology and simultaneously measures the following proteins: Interleukin-1 $\beta$ (IL-1 $\beta$ ), IL-1 receptor antagonist (IL-1ra), IL-2, IL-4, IL-5, IL-6, IL-7, IL-8, IL-9, IL-10, IL-12 (p70), IL-13, IL-15, IL-17, basic fibroblast growth factor (bFGF), eotaxin, granulocyte colony-stimulating factor (G-CSF), granulocyte macrophage colony-stimulating factor (GM-CSF), interferon- $\gamma$ (IFN- $\gamma$ ), IFN-inducible protein 10 (IP-10), monocyte chemoattractant protein-1 (MCP-1; MCAF), 
macrophage inflammatory protein-1 $\alpha$ (MIP-1 $\alpha$ ), MIP-1 $\beta$, platelet-derived growth factor-BB (PDGF-BB), regulated on activation normal T-cell expressed and secreted (RANTES), tumor necrosis factor- $\alpha$ (TNF- $\alpha$ ), and vascular endothelial growth factor (VEGF). Briefly, anticytokine antibody-conjugated beads were added to individual wells of a 96-well filter plate and washed using vacuum filtration. After washing, $50 \mu \mathrm{L}$ of prediluted standards (range = $32,000-1.95 \mathrm{pg} / \mathrm{mL}$ ) or cell-free conditioned medium from Beas $2 \mathrm{~B}$ cells ( $n=3 /$ group) was added, and the filter plate shaken at $300 \mathrm{rpm}$ for $30 \mathrm{~min}$ at room temperature. Thereafter, the filter plate was washed, and $25 \mu \mathrm{L}$ of prediluted multiplex detection antibody was added for $30 \mathrm{~min}$. After washing, $50 \mu \mathrm{L}$ of prediluted streptavidin-conjugated phycoerythrin was added for $10 \mathrm{~min}$ followed by an additional wash and the addition of $120 \mu \mathrm{L}$ of Bioplex assay buffer to each well. The filter plate was analyzed using the Bioplex protein array system, and concentrations of each cytokine were determined using Bioplex Manager Version 3.0 software (Bio-Rad, Hercules, CA). Data are expressed as pg of cytokine/mL of conditioned medium.

\section{Statistical analyses}

Statistical analysis of results from cell viability and Bioplex analysis were evaluated by analysis of variance (ANOVA) using the Student Neuman-Keul's procedure for adjustment of multiple pair-wise comparisons between treatment groups. Significant differences in gene expression values determined by qRT-PCR were evaluated using a Student's t-test. Differences with $\mathrm{p}$ values $<0.05$ were considered statistically significant. 


\section{REFERENCES}

1. Mundt KA, Birk T, Parsons W, Borsch-Galetke E, Siegmund K, Heavner K, Guldner K: Respirable crystalline silica exposure-response evaluation of silicosis morbidity and lung cancer mortality in the German porcelain industry cohort. J Occup Environ Med 2011, 53:282-289.

2. Cox Jr LA: An Exposure-Response Threshold for Lung Diseases and Lung Cancer Caused by Crystalline Silica. Risk Anal 2011.

3. Rimal B, Greenberg AK, Rom WN: Basic pathogenetic mechanisms in silicosis: current understanding. Curr Opin Pulm Med 2005, 11:169-173.

4. Gamble JF: Crystalline silica and Lung cancer: A critical review of the occupational epidemiology literature of exposure-response studies testing this hypothesis. Crit Rev Toxicol 2011, 41:404-465.

5. Cassidy A, t Mannetje A, van Tongeren M, Field JK, Zaridze D, Szeszenia-Dabrowska N, Rudnai P, Lissowska J, Fabianova E, Mates D, et al: Occupational exposure to crystalline silica and risk of lung cancer: a multicenter case-control study in Europe. Epidemiology 2007, 18:36-43.

6. Finkelstein MM: Silica, silicosis, and lung cancer: a risk assessment. Am J Ind Med 2000, 38:818.

7. Flanagan ME, Seixas N, Majar M, Camp J, Morgan M: Silica dust exposures during selected construction activities. AIHA J (Fairfax, Va) 2003, 64:319-328.

8. Rappaport SM, Goldberg M, Susi P, Herrick RF: Excessive exposure to silica in the US construction industry. Ann Occup Hyg 2003, 47:111-122.

9. Cocco P: The long and winding road from silica exposure to silicosis and other health effects. Occup Environ Med 2003, 60:157-158.

10. Absher MP, Trombley L, Hemenway DR, Mickey RM, Leslie KO: Biphasic cellular and tissue response of rat lungs after eight-day aerosol exposure to the silicon dioxide cristobalite. Am J Pathol $1989,134: 1243-1251$.

11. Mossman BT, Churg A: Mechanisms in the pathogenesis of asbestosis and silicosis. Am J Respir Crit Care Med 1998, 157:1666-1680.

12. Riva DR, Magalhaes CB, Lopes AA, Lancas T, Mauad T, Malm O, Valenca SS, Saldiva PH, Faffe DS, Zin WA: Low dose of fine particulate matter (PM2.5) can induce acute oxidative stress, inflammation and pulmonary impairment in healthy mice. Inhal Toxicol 2011, 23:257-267.

13. Carter JM, Driscoll KE: The role of inflammation, oxidative stress, and proliferation in silicainduced lung disease: a species comparison. J Environ Pathol Toxicol Oncol 2001, 20 Suppl 1:33-43.

14. Donaldson K, Stone V, Duffin R, Clouter A, Schins R, Borm P: The quartz hazard: effects of surface and matrix on inflammogenic activity. J Environ Pathol Toxicol Oncol 2001, 20 Suppl 1:109118.

15. Knaapen AM, Albrecht C, Becker A, Hohr D, Winzer A, Haenen GR, Borm PJ, Schins RP: DNA damage in lung epithelial cells isolated from rats exposed to quartz: role of surface reactivity and neutrophilic inflammation. Carcinogenesis 2002, 23:1111-1120. 
16. Porter DW, Ramsey D, Hubbs AF, Battelli L, Ma J, Barger M, Landsittel D, Robinson VA, McLaurin J, Khan A, et al: Time course of pulmonary response of rats to inhalation of crystalline silica: histological results and biochemical indices of damage, lipidosis, and fibrosis. J Environ Pathol Toxicol Oncol 2001, 20 Suppl 1:1-14.

17. Rao KM, Porter DW, Meighan T, Castranova V: The sources of inflammatory mediators in the lung after silica exposure. Environ Health Perspect 2004, 112:1679-1686.

18. Velan GM, Kumar RK, Cohen DD: Pulmonary inflammation and fibrosis following subacute inhalational exposure to silica: determinants of progression. Pathology 1993, 25:282-290.

19. Warheit DB, McHugh TA, Hartsky MA: Differential pulmonary responses in rats inhaling crystalline, colloidal or amorphous silica dusts. Scand J Work Environ Health 1995, 21 Suppl 2:19-21.

20. Ovrevik J, Lag M, Schwarze P, Refsnes M: p38 and Src-ERK1/2 pathways regulate crystalline silica-induced chemokine release in pulmonary epithelial cells. Toxicol Sci 2004, 81:480-490.

21. Ramos-Nino ME, Haegens A, Shukla A, Mossman BT: Role of mitogen-activated protein kinases (MAPK) in cell injury and proliferation by environmental particulates. Mol Cell Biochem 2002, 234235:111-118.

22. Albrecht C, Borm PJ, Adolf B, Timblin CR, Mossman BT: In vitro and in vivo activation of extracellular signal-regulated kinases by coal dusts and quartz silica. Toxicol Appl Pharmacol 2002, $184: 37-45$.

23. Hubbard AK, Timblin CR, Rincon M, Mossman BT: Use of transgenic luciferase reporter mice to determine activation of transcription factors and gene expression by fibrogenic particles. Chest 2001, 120:24S-25S.

24. Hubbard AK, Timblin CR, Shukla A, Rincon M, Mossman BT: Activation of NF-kappaBdependent gene expression by silica in lungs of luciferase reporter mice. Am J Physiol Lung Cell Mol Physiol 2002, 282:L968-975.

25. Beck-Speier I, Dayal N, Karg E, Maier KL, Schumann G, Schulz H, Semmler M, Takenaka S, Stettmaier K, Bors W, et al: Oxidative stress and lipid mediators induced in alveolar macrophages by ultrafine particles. Free Radic Biol Med 2005, 38:1080-1092.

26. Brown DM, Wilson MR, MacNee W, Stone V, Donaldson K: Size-dependent proinflammatory effects of ultrafine polystyrene particles: a role for surface area and oxidative stress in the enhanced activity of ultrafines. Toxicol Appl Pharmacol 2001, 175:191-199.

27. Mossman BT, Shukla A, Fukagawa NK: Highlight Commentary on "Oxidative stress and lipid mediators induced in alveolar macrophages by ultrafine particles". Free Radic Biol Med 2007, 43:504505.

28. Guthrie GD, Jr., Heaney PJ: Mineralogical characteristics of silica polymorphs in relation to their biological activities. Scand J Work Environ Health 1995, 21 Suppl 2:5-8.

29. Hillegass JM, Shukla A, MacPherson MB, Bond JP, Steele C, Mossman BT: Utilization of gene profiling and proteomics to determine mineral pathogenicity in a human mesothelial cell line (LP9/TERT-1). J Toxicol Environ Health A 2010, 73:423-436. 
30. Hillegass JM, Shukla A, MacPherson MB, Lathrop SA, Alexeeva V, Perkins TN, van der Vliet A, Vacek PM, Gunter ME, Mossman BT: Mechanisms of oxidative stress and alterations in gene expression by Libby six-mix in human mesothelial cells. Part Fibre Toxicol 2010, 7:26.

31. Shukla A, MacPherson MB, Hillegass J, Ramos-Nino ME, Alexeeva V, Vacek PM, Bond JP, Pass HI, Steele C, Mossman BT: Alterations in gene expression in human mesothelial cells correlate with mineral pathogenicity. Am J Respir Cell Mol Biol 2009, 41:114-123.

32. Fubini B, Giamello E, Volante M, Bolis V: Chemical functionalities at the silica surface determining its reactivity when inhaled. Formation and reactivity of surface radicals. Toxicol Ind Health 1990, 6:571-598.

33. Fubini B, Zanetti G, Altilia S, Tiozzo R, Lison D, Saffiotti U: Relationship between surface properties and cellular responses to crystalline silica: studies with heat-treated cristobalite. Chem Res Toxicol 1999, 12:737-745.

34. Ghio AJ, Kennedy TP, Whorton AR, Crumbliss AL, Hatch GE, Hoidal JR: Role of surface complexed iron in oxidant generation and lung inflammation induced by silicates. Am J Physiol 1992, 263:L511-518.

35. Vallyathan V, Shi XL, Dalal NS, Irr W, Castranova V: Generation of free radicals from freshly fractured silica dust. Potential role in acute silica-induced lung injury. Am Rev Respir Dis 1988, 138:1213-1219.

36. Fubini B, Hubbard A: Reactive oxygen species (ROS) and reactive nitrogen species (RNS) generation by silica in inflammation and fibrosis. Free Radic Biol Med 2003, 34:1507-1516.

37. Fubini B, Fenoglio I, Elias Z, Poirot O: Variability of biological responses to silicas: effect of origin, crystallinity, and state of surface on generation of reactive oxygen species and morphological transformation of mammalian cells. J Environ Pathol Toxicol Oncol 2001, 20 Suppl 1:95-108.

38. Johnston CJ, Driscoll KE, Finkelstein JN, Baggs R, O'Reilly MA, Carter J, Gelein R, Oberdorster G: Pulmonary chemokine and mutagenic responses in rats after subchronic inhalation of amorphous and crystalline silica. Toxicol Sci 2000, 56:405-413.

39. Warheit DB: Inhaled amorphous silica particulates: what do we know about their toxicological profiles? J Environ Pathol Toxicol Oncol 2001, 20 Suppl 1:133-141.

40. McDermott JE, Archuleta M, Thrall BD, Adkins JN, Waters KM: Controlling the response: predictive modeling of a highly central, pathogen-targeted core response module in macrophage activation. PLoS One 2011, 6:e14673.

41. Heintz NH, Janssen YM, Mossman BT: Persistent induction of c-fos and c-jun expression by asbestos. Proc Natl Acad Sci U S A 1993, 90:3299-3303.

42. Belitskaya-Levy I, Hajjou M, Su WC, Yie TA, Tchou-Wong KM, Tang MS, Goldberg JD, Rom WN: Gene profiling of normal human bronchial epithelial cells in response to asbestos and benzo(a)pyrene diol epoxide (BPDE). J Environ Pathol Toxicol Oncol 2007, 26:281-294.

43. Barrett EG, Johnston C, Oberdorster G, Finkelstein JN: Silica-induced chemokine expression in alveolar type II cells is mediated by TNF-alpha. Am J Physiol 1998, 275:L1110-1119.

44. Hetland RB, Schwarze PE, Johansen BV, Myran T, Uthus N, Refsnes M: Silica-induced cytokine release from A549 cells: importance of surface area versus size. Hum Exp Toxicol 2001, 20:46-55. 
45. Ovrevik J, Lag M, Holme JA, Schwarze PE, Refsnes M: Cytokine and chemokine expression patterns in lung epithelial cells exposed to components characteristic of particulate air pollution. Toxicology 2009, 259:46-53.

46. Ovrevik J, Refsnes M, Namork E, Becher R, Sandnes D, Schwarze PE, Lag M: Mechanisms of silica-induced IL-8 release from A549 cells: initial kinase-activation does not require EGFR activation or particle uptake. Toxicology 2006, 227:105-116.

47. Ovrevik J, Myran T, Refsnes M, Lag M, Becher R, Hetland RB, Schwarze PE: Mineral particles of varying composition induce differential chemokine release from epithelial lung cells: importance of physico-chemical characteristics. Ann Occup Hyg 2005, 49:219-231.

48. Shukla A, Timblin CR, Hubbard AK, Bravman J, Mossman BT: Silica-induced activation of cJun-NH2-terminal amino kinases, protracted expression of the activator protein-1 proto-oncogene, fra1, and S-phase alterations are mediated via oxidative stress. Cancer Res 2001, 61:1791-1795.

49. Nymark P, Lindholm PM, Korpela MV, Lahti L, Ruosaari S, Kaski S, Hollmen J, Anttila S, Kinnula VL, Knuutila S: Gene expression profiles in asbestos-exposed epithelial and mesothelial lung cell lines. BMC Genomics 2007, 8:62.

50. Hengstermann A, Muller T: Endoplasmic reticulum stress induced by aqueous extracts of cigarette smoke in 3T3 cells activates the unfolded-protein-response-dependent PERK pathway of cell survival. Free Radic Biol Med 2008, 44:1097-1107.

51. Davis GS, Pfeiffer LM, Hemenway DR, Rincon M: Interleukin-12 is not essential for silicosis in mice. Part Fibre Toxicol 2006, 3:2.

52. Beamer CA, Migliaccio CT, Jessop F, Trapkus M, Yuan D, Holian A: Innate immune processes are sufficient for driving silicosis in mice. J Leukoc Biol 2010, 88:547-557.

53. Khalaf H, Jass J, Olsson PE: Differential cytokine regulation by NF-kappaB and AP-1 in Jurkat T-cells. BMC Immunol 2010, 11:26.

54. Kube D, Sontich U, Fletcher D, Davis PB: Proinflammatory cytokine responses to P. aeruginosa infection in human airway epithelial cell lines. Am J Physiol Lung Cell Mol Physiol 2001, 280:L493502.

55. Wedzicha JA, Seemungal TA, MacCallum PK, Paul EA, Donaldson GC, Bhowmik A, Jeffries DJ, Meade TW: Acute exacerbations of chronic obstructive pulmonary disease are accompanied by elevations of plasma fibrinogen and serum IL-6 levels. Thromb Haemost 2000, 84:210-215.

56. Stecenko AA, King G, Torii K, Breyer RM, Dworski R, Blackwell TS, Christman JW, Brigham KL: Dysregulated cytokine production in human cystic fibrosis bronchial epithelial cells. Inflammation 2001, 25:145-155.

57. Zhai R, Ge X, Li H, Tang Z, Liao R, Kleinjans J: Differences in cellular and inflammatory cytokine profiles in the bronchoalveolar lavage fluid in bagassosis and silicosis. Am J Ind Med 2004, 46:338-344.

58. Miller MD, Krangel MS: Biology and biochemistry of the chemokines: a family of chemotactic and inflammatory cytokines. Crit Rev Immunol 1992, 12:17-46.

59. Hamilton JA: Colony-stimulating factors in inflammation and autoimmunity. Nat Rev Immunol 2008, 8:533-544. 
60. Yao W, Feng FF, Jiao J, Wang N: [Expression level and significance of TGF-beta1, PDGF, CTGF in serum of patients with pneumoconiosis]. Sichuan Da Xue Xue Bao Yi Xue Ban 2006, 37:754$756,793$.

61. Brandt-Rauf PW, Smith S, Hemminki K, Koskinen H, Vainio H, Niman H, Ford J: Serum oncoproteins and growth factors in asbestosis and silicosis patients. Int J Cancer 1992, 50:881-885.

62. Ando M, Miyazaki E, Ito T, Hiroshige S, Nureki SI, Ueno T, Takenaka R, Fukami T, Kumamoto T: Significance of serum vascular endothelial growth factor level in patients with idiopathic pulmonary fibrosis. Lung 2010, 188:247-252.

63. Stockmann C, Kerdiles Y, Nomaksteinsky M, Weidemann A, Takeda N, Doedens A, TorresCollado AX, Iruela-Arispe L, Nizet V, Johnson RS: Loss of myeloid cell-derived vascular endothelial growth factor accelerates fibrosis. Proc Natl Acad Sci U S A 2010, 107:4329-4334.

64. Bodo M, Baroni T, Bellocchio S, Calvitti M, Lilli C, D'Alessandro A, Muzi G, Lumare A, Abbritti G: Bronchial epithelial cell matrix production in response to silica and basic fibroblast growth factor. Mol Med 2001, 7:83-92.

65. Herseth JI, Volden V, Schwarze PE, Lag M, Refsnes M: IL-1beta differently involved in IL-8 and FGF-2 release in crystalline silica-treated lung cell co-cultures. Part Fibre Toxicol 2008, 5:16.

66. Selige J, Tenor H, Hatzelmann A, Dunkern T: Cytokine-dependent balance of mitogenic effects in primary human lung fibroblasts related to cyclic AMP signaling and phosphodiesterase 4 inhibition. J Cell Physiol 2010, 223:317-326.

67. Shirakihara T, Horiguchi K, Miyazawa K, Ehata S, Shibata T, Morita I, Miyazono K, Saitoh M: TGF-beta regulates isoform switching of FGF receptors and epithelial-mesenchymal transition. EMBO J 2011, 30:783-795.

68. Khalil N, Xu YD, O'Connor R, Duronio V: Proliferation of pulmonary interstitial fibroblasts is mediated by transforming growth factor-beta1-induced release of extracellular fibroblast growth factor-2 and phosphorylation of p38 MAPK and JNK. J Biol Chem 2005, 280:43000-43009.

69. Hamada H, Vallyathan V, Cool CD, Barker E, Inoue Y, Newman LS: Mast cell basic fibroblast growth factor in silicosis. Am J Respir Crit Care Med 2000, 161:2026-2034.

70. Zhou R, Tardivel A, Thorens B, Choi I, Tschopp J: Thioredoxin-interacting protein links oxidative stress to inflammasome activation. Nat Immunol 2010, 11:136-140.

71. Dostert C, Petrilli V, Van Bruggen R, Steele C, Mossman BT, Tschopp J: Innate immune activation through Nalp3 inflammasome sensing of asbestos and silica. Science 2008, 320:674-677.

72. Timblin CR, Shukla A, Berlanger I, BeruBe KA, Churg A, Mossman BT: Ultrafine airborne particles cause increases in protooncogene expression and proliferation in alveolar epithelial cells. Toxicol Appl Pharmacol 2002, 179:98-104.

73. Huang YC, Karoly ED, Dailey LA, Schmitt MT, Silbajoris R, Graff DW, Devlin RB: Comparison of gene expression profiles induced by coarse, fine, and ultrafine particulate matter. $J$ Toxicol Environ Health A 2011, 74:296-312. 
74. De G, Karmakar B, Ganguli D: Hydrolysis-condensation reactions of TEOS in the presence of acetic acid leading to the generation of glass-like silica microspheres in solution at room temperature. J Mater Chem 2000, 10:2289-2293.

75. Janssen YM, Marsh JP, Absher MP, Hemenway D, Vacek PM, Leslie KO, Borm PJ, Mossman BT: Expression of antioxidant enzymes in rat lungs after inhalation of asbestos or silica. $J$ Biol Chem 1992, 267:10625-10630.

76. Kruk M, Antochshuk V, Jaroniec M, Sayari A: New approach to evaluate pore size distributions and surface areas for hydrophobic mesoporous solids. J Phys Chem B 1999, 103:10670-10678.

77. Kruk M, Jaroniec M, Sakamoto Y, Terasaki O, Ryoo R, Ko CH: Determination of pore size and pore wall structure of MCM-41 by using nitrogen adsorption, transmission electron microscopy, and X-ray diffraction. $J$ Phys Chem B 2000, 104:292-301.

78. Kruk M, Jaroniec M, Sayari A: Application of large pore MCM-41 molecular sieves to improve pore size analysis using nitrogen adsorption measurements. Langmuir 1997, 13:6267-6273.

79. Hillegass JM, Shukla A, Lathrop SA, MacPherson MB, Fukagawa NK, Mossman BT: Assessing nanotoxicity in cells in vitro. Wiley Interdiscip Rev Nanomed Nanobiotechnol 2010, 2:219-231.

80. Shukla A, Lounsbury KM, Barrett TF, Gell J, Rincon M, Butnor KJ, Taatjes DJ, Davis GS, Vacek P, Nakayama KI, et al: Asbestos-induced peribronchiolar cell proliferation and cytokine production are attenuated in lungs of protein kinase C-delta knockout mice. Am J Pathol 2007, 170:140-151.

81. Sabo-Attwood T, Ramos-Nino M, Bond J, Butnor KJ, Heintz N, Gruber AD, Steele C, Taatjes DJ, Vacek P, Mossman BT: Gene expression profiles reveal increased mClca3 (Gob5) expression and mucin production in a murine model of asbestos-induced fibrogenesis. Am J Pathol 2005, 167:12431256. 


\section{SUPPLEMENTARY MATERIAL}

A

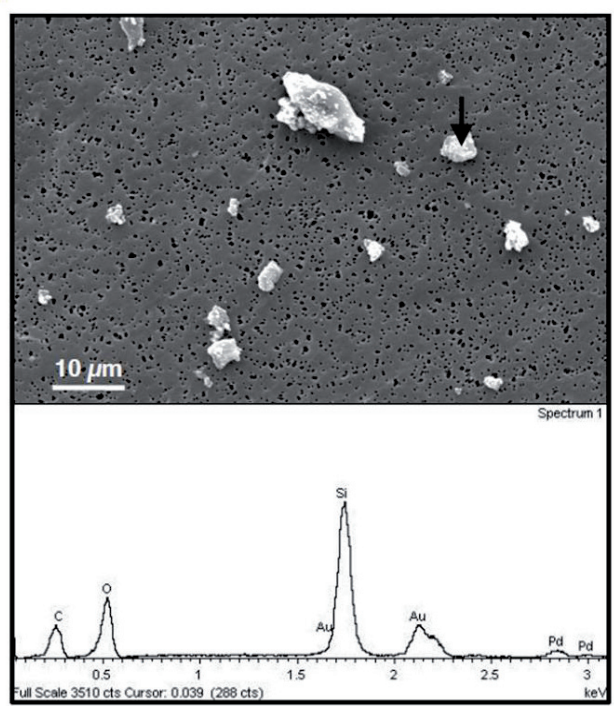

B

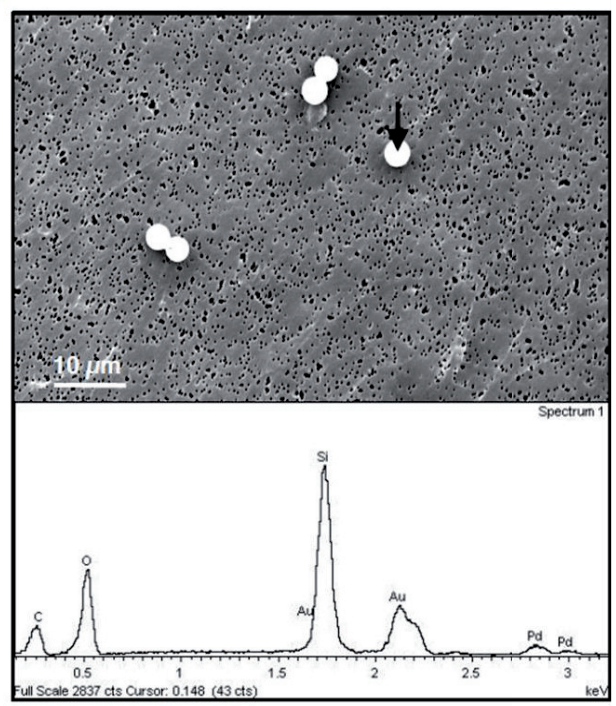

Supplementary Figure 1: SEM imaging and EDS analysis of silica particles. SEM images if (A) cristobalite silica and (B) amorphous silica particles with respectful EDS spectra. Images are at a magnification of $1500 X$ and scale bars equal to $10 \mu \mathrm{m}$. Black arrows indicate the points analyzed by $E D S$, representing each silica particle type. Silicon (Si) and oxygen $(O)$ peaks of each particle type indicate both are pure silica particles. Gold (Au) and palladium (Pd) peaks are present due to the gold and palladium sputter-coating, carbon $(C)$ is present because of carbon paint used to mount particle filters. 


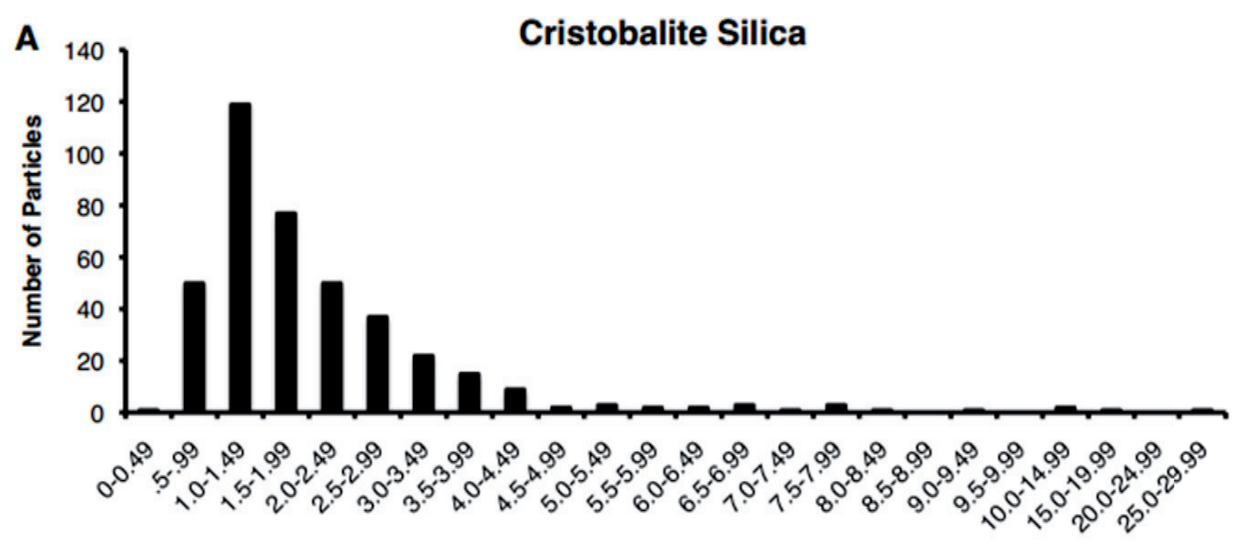

B Amorphous Silica

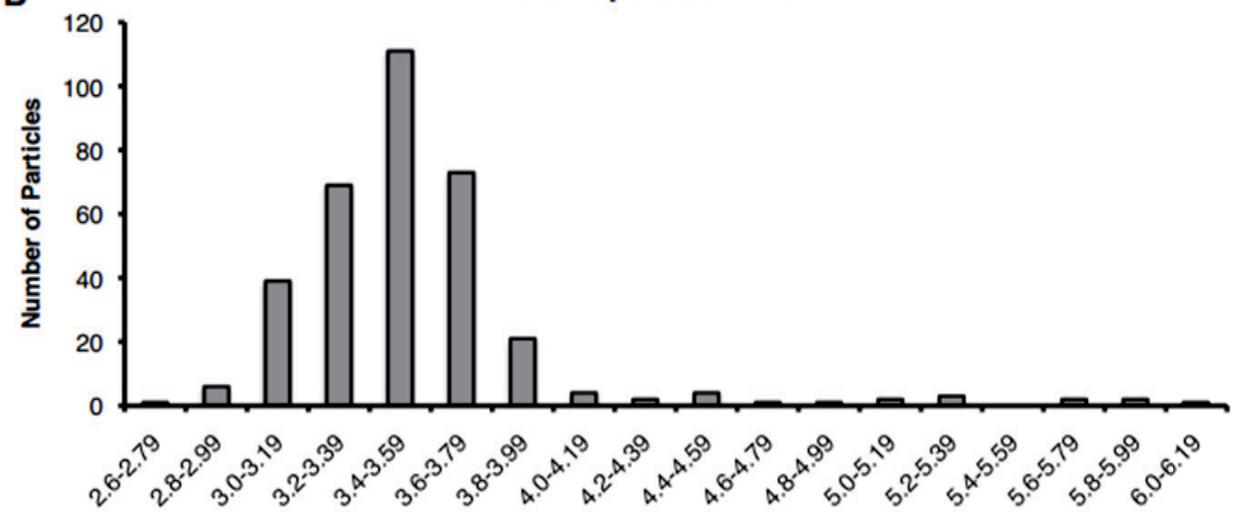

Particle Diameter $(\mu \mathrm{m})$

Supplementary figure 2: Silica particle size distrbution. Histograms represent size-distribution of cristobalite silica particles (A) and amorphous silica particles (B). Particles were filtered and imaged

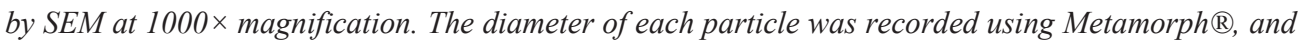
300-400 particles (5 fields/stub) were measured for each type of silica particle. 


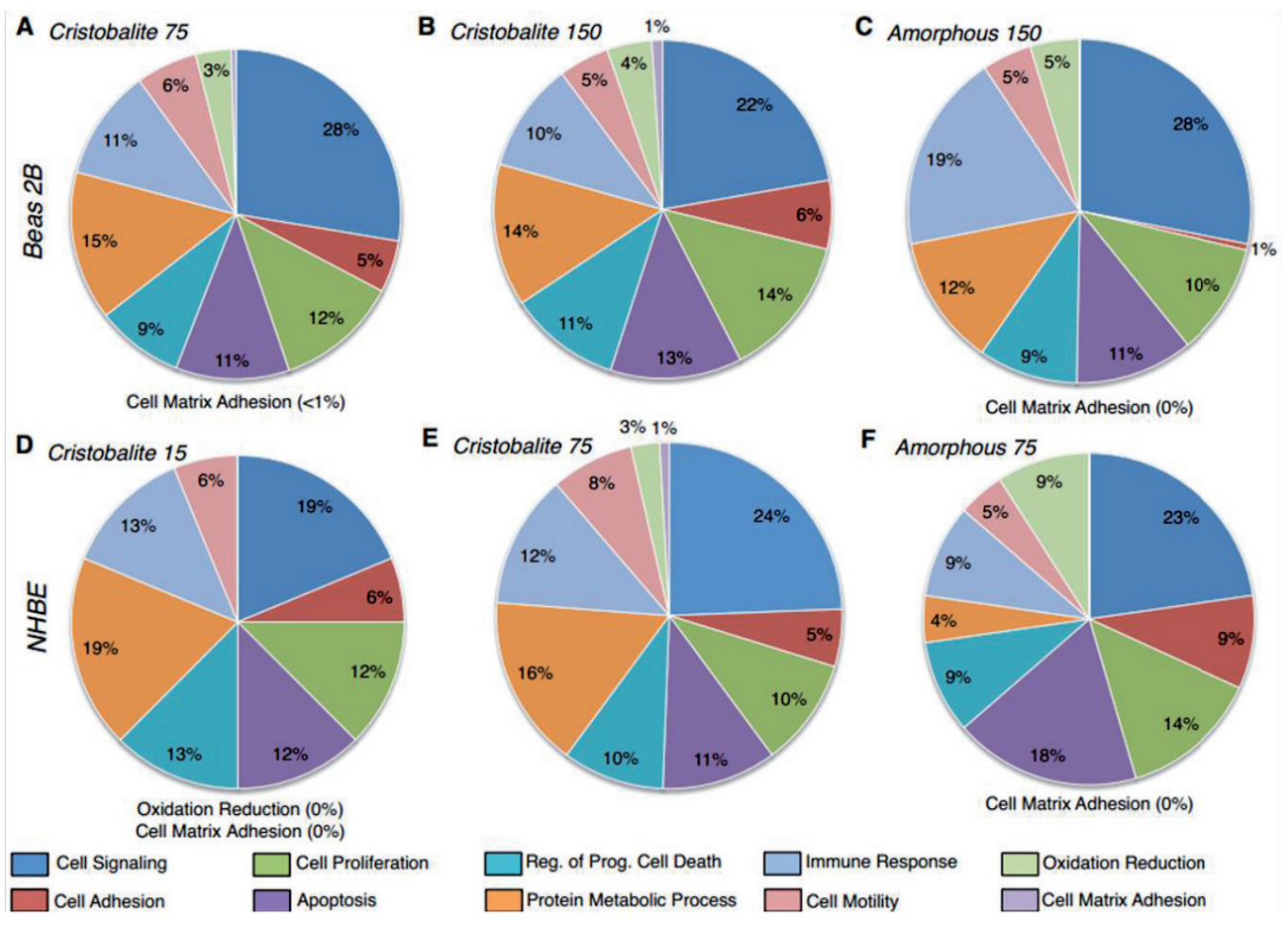

Supplementary figure 3: Gene ontology of BEAS $2 B$ and NHBE Exposed to Silica Particles for 24 $\boldsymbol{h}$ (\% Total/Category). Pie charts represents gene ontology analysis of alterations in gene expression of BEAS $2 B$ cells $(\boldsymbol{A}-\boldsymbol{C})$ exposed to $(\boldsymbol{A})$ cristobalite $75 \times 10^{6} \mu \mathrm{m} 2 / \mathrm{cm} 2$, (B) cristobalite $150 \times 10^{6}$ $\mu \mathrm{m} 2 / \mathrm{cm} 2$ and $(\boldsymbol{C})$ amorphous silica $150 \times 10^{6} \mu \mathrm{m} 2 / \mathrm{cm} 2$. Gene ontology of NHBE cells (D-F) exposed to (D) cristobalite $15 \times 10^{6} \mu \mathrm{m} 2 / \mathrm{cm} 2$, (E) cristobalite $75 \times 10^{6} \mu \mathrm{m} 2 / \mathrm{cm} 2$ and amorphous silica $75 X$ $10^{6} \mu \mathrm{m} 2 / \mathrm{cm} 2$. Ten categories of interest were analyzed and charts represent the percent (\%) of total genes altered in each category for each exposure group. 



\title{
CHAPTER 5
}

\section{IMMUNE HOMEOSTASIS IN EPITHELIAL CELLS: EVIDENCE AND ROLE OF INFLAMMASOME SIGNALING REVIEWED}

\author{
Paul M Peeters ${ }^{1}$, Emiel FM Wouters ${ }^{1}$, Niki L Reynaert ${ }^{1}$ \\ ${ }^{1}$ Department of Respiratory Medicine, Maastricht University Medical Centre, Maastricht University, \\ Maastricht, The Netherlands.
}

\section{Journal of IMMUNOLOGY RESEARCH (In Revision)}

\begin{abstract}
The epithelium regulates the interaction between the noxious xenogenous, as well as the microbial environment and the immune system, not only by providing a barrier but also by expressing a number of immunoregulatory membrane receptors, intracellular danger sensors and their downstream effectors. Amongst these are a number of inflammasome sensor subtypes, which have been initially characterized in myeloid cells and described to be activated upon assembly into multiprotein complexes by microbial and environmental triggers. This review compiles a vast amount of literature that supports a pivotal role for inflammasomes in the various epithelial barriers of the human body as essential factors maintaining immune signaling and homeostasis.
\end{abstract}





\section{INTRODUCTION}

Inflammation is an extremely complex and fascinating weapon in mammalian physiology. It is the body's immediate and carefully orchestrated response to pathogens, noxious stimuli or physical injury. In addition, responsiveness to plasma- and cell-derived inflammatory mediators reflects a more general role for inflammation in restoring functionality of the system to basal homeostatic set points. The process, by which acute inflammation is initiated and develops via molecular and cellular pathways is well defined (1). In case the body does not succeed to eliminate or neutralize this condition over time, a chronic inflammatory state arises and resets the body's reference points and will become maladaptive. This implicates elevated concentrations of cytokines and chemokines including interleukin (IL)-1 $\beta$, IL-1 $\alpha$, IL6, IL-8, IL-10, IL-18, TNF- $\alpha$ and alarmins such as high-mobility group box 1 (HMGB1). These molecules have also been proven to be involved in the progression of chronic inflammatory disorders, infections, fibrotic diseases as well as in cancer, autoimmune and ageing-associated disorders.

It is demonstrated that IL-1, IL-18 and HMGB1 driven inflammation, through inflammasome activation, is initiated by recognition of endogenous or exogenous danger signals. IL- $1 \beta$, one of the main classic instigators of inflammation is, together with IL-18, released from the cell upon activation of the inflammasome. It has the ability to affect various biological properties and has several roles in the proinflammatory response, including activation of the endothelium and leukocytes. Back in 1984 it was predicted that IL-1 was responsible for many of the acute responses to infection and inflammation (2). IL-18, a member of the IL-1 cytokine super family, is recognized as an important regulator of innate and acquired immune responses. Its importance is derived from its prominent biological property of inducing interferon (IFN) $\gamma$. IL-18 is expressed at sites of chronic inflammation, in autoimmune diseases, in a variety of cancers, and in the context of numerous infectious diseases (3). HMGB1 is a multifunctional protein; its earliest functions were described as a non-histone DNA-binding nuclear protein. It facilitates DNA transcription, replication, and repair. Secreted, it has an important danger signaling and inflammation-promoting activity. HMGB1 is unconventionally released such as for instance under caspase-1 activated conditions following a number of posttranslational modifications (4). It forms highly inflammatory complexes with single stranded (ss) DNA, lipopolysacharide (LPS), IL-1 $\beta$ and nucleosomes, and interacts with toll-like receptor (TLR) 9, TLR4, IL-1R and TLR2 and the receptor for advanced glycation end-products (RAGE). It has also been reported that HMGB1 signals to induce the nuclear translocation of nuclear factor kappa-light-chain-enhancer of activated $\mathrm{B}$ cells $(\mathrm{NF}-\mathrm{\kappa B})$ resulting in an enhanced production and release of proinflammatory cytokines, including TNF- $\alpha$ and IL-1 $\beta$. Activation of inflammasome-dependent mediator release is typically a very potent reaction and by virtue of the potentially destructive proinflammatory effects of uncontrolled cytokine and alarmin release their reduction and shutdown mechanisms (e.g. IL-1 receptor antagonist (IL-1RA) signaling) should therefore be tightly regulated by innate sensors.

To date, a number of cytosolic receptors are successful in the recognition of conserved molecular patterns termed pathogen- or danger-associated molecular patterns (PAMPs or DAMPs), by initiating the formation of an inflammasome (5). These include the nucleotide- 
binding oligomerization domain receptors, in short NOD-like receptor (NLR) protein family members NLRP1, NLRP3, NLRP6, NLRP7, NLRP12, NLRC4 and NLRC5 as well as the non-NLR pyrin and HIN200 domain-containing (PYHIN) protein family members absent in melanoma 2 (AIM2), myeloid nuclear differentiation antigen (MNDA), Interferon inducible protein X (IFIX) and Interferon alpha-inducible protein 16 (IFI16) which are all able to oligomerize into a functional inflammasome (6-8). This implies the formation of a multiprotein complex consisting otherwise also of an adaptor protein, apoptosis-like speck protein containing a CARD (ASC) and IL-1 $\beta$ converting enzyme (ICE, Caspase-1), the enzyme responsible for maturation of proinflammatory cytokines (9). Upon this assembly, caspase-1 undergoes autocatalytic activation into heterotetramers, which further enables the cleavage of its substrates pro-IL-1 $\beta$ and pro-IL-18 into mature IL-1 $\beta$ and IL-18, and the unconventional release of fibroblast growth factor (bFGF) and alarmins by the cell as is illustrated in Figure 1 (10). In addition, caspase-1 is associated with the release of IL-1 $\alpha$ (4), although these mechanisms are poorly understood. Inflammasome activation is thus linked to the most important mediators of inflammation. Moreover, their release may be accompanied by pyroptosis, an incompletely characterized proinflammatory mode of cell death (11). Upon activation by a myriad of signaling pathways these inflammasome-dependent mediators are strongly expressed by monocytes, tissue macrophages and dendritic cells, but are also produced by B lymphocytes, natural killer (NK) cells and epithelial cells. As mediators of the acute phase of inflammation, the inflammasomes and the cytokines and alarmins they activate and release play a key and well-controlled role in innate immunity of the lung mucosa and interstitial microenvironment as it is extremely important for the immune system to react to invading pathogens. It is however equally important that inflammasomes distinguish pathogenic from non-pathogenic commensals, implying that a disturbance in normal danger signaling through the inflammasome can acts as a master switch between tolerance and sensitization in many actively participating tissues (12-16).

The activation of inflammasomes in myeloid innate immune cells as well as their contribution to acute and chronic inflammatory diseases has been characterized profoundly over the past 12 years by in vivo and in vitro research (17). Nevertheless, we are still far away from understanding how these molecules actually become activated, how they exert their function, and how they can be targeted in therapy. Aside from the specialized cells of the immune system that evolutionary developed to protect organ systems, most foreign pathogens and noxious stimuli are also encountered by epithelial cells in a barrier lining the organs that are in most proximal contact with the exterior environment (18). These are the tissue specific mucosae of the skin, the lung, gut and urogenital tract, as well as non-keratinizing squamous epithelial cells of the oral mucosa.

The epithelium is more and more appreciated to be less passive than assumed before and evidence is mounting that it participates not only in receiving and relaying inflammatory signals, but functions as an initial sensor of danger and executor of the response as well. We therefore review here the literature on the presence and functionality of inflammasomes in epithelial cells of the various organs exposed to the exterior milieu in response to reported insults. The aim of this review is to deliver a better understanding of inflammatory responses of first line barrier epithelium in multiple organs and mucosal immunity, and encourage 
further laboratory research to dissect out the role of epithelial inflammasomes to these processes, with more effective therapies for the numerous debilitating diseases with an acute and chronic inflammatory component as the ultimate goal.

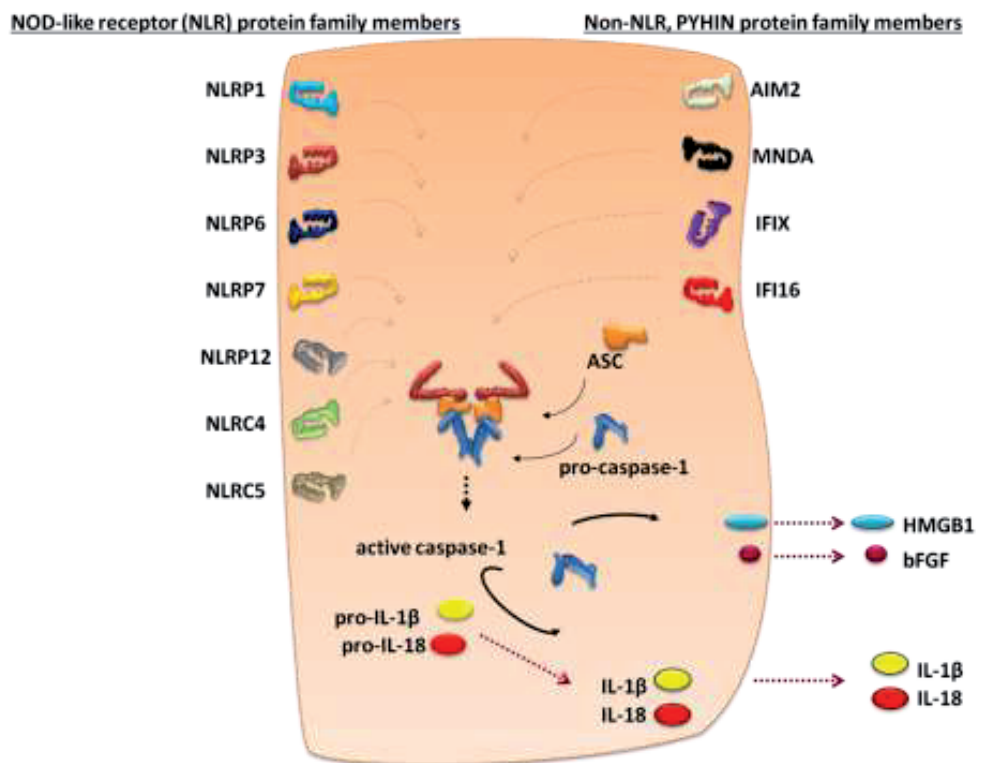

Figure 1: Schematic representation of all intracellular nucleotide-binding oligomerization domain receptors, in short NOD-like receptor (NLR) and pyrin and HIN200 domain-containing (PYHIN) inflammasome members, that are each able to assemble with the protease caspase-1 via the adaptor molecule apoptosis-like speck protein containing a CARD (ASC) when triggered. This allows the activated enzyme to cleave and mature proinflammatory cytokines interleukin (IL)-1 $\beta$ and IL-18 as well as to induce the unconventional release of basic fibroblast growth factor (bFGF) and highmobility group box 1 (HMGB1).

\section{SKIN}

In addition to its properties as a physical barrier, the skin has many active defense mechanisms. Keratinocytes can detect microbial or nonmicrobial danger signals and elicit an immune response prior to the infiltration of myeloid cells (19). Although the action and importance of IL-1 $\beta$, IL-18 and HMGB1 in inflammatory skin disorders is not completely understood, dysregulation of these inflammasome-dependent molecules is an attractive concept that might play a role in many inflammatory abnormalities of the skin. In 1990 already, protein levels of immunoreactive IL-1 $\beta$ were shown to be elevated in psoriatic lesions whereas the amount released by normal keratomes or cultured keratinocytes was undetectable. The presence of IL-1 $\beta$ was suggested to be due to a novel mechanism of posttranslational processing in the epidermis (20). This mechanism was identified in 1997 as caspase-1 dependent cleavage which could be induced in human keratinocytes in response to inflammatory and immunologic stimuli (21).

Later, keratinocytes of the non-diseased skin were scarcely stained positive for NLRP1 and NLRP3 (22) and AIM2-like receptor (ALR) inflammasomes (23). These expression patterns 
suggest that multiple inflammasomes are likely to play a role in the first line of defense against noxious molecules. With respect to different functional inflammasomes in the skin, human keratinocytes express AIM2 and respond to poly (dA:dT) dsDNA with IL-1 $\beta$ secretion (23). Recently, these findings have been supported by detection of active IL-1 $\beta$ and cleaved caspase-1 in human papillomavirus (HPV) infected skin, suggesting inflammasome activation by viral DNA (24). Watanabe $\mathrm{H}$ et al. also demonstrated that the NLRP3 inflammasome is present and can be activated in keratinocytes (16) as in animal models of contact hypersensitivity this inflammasome was identified as a key regulator of innate immunity (16). Keratinocytes are obviously also barrier cells against environmental pollutants such as $\mathrm{TiO}_{2}$ and $\mathrm{SiO}_{2}$. It was shown that these environmental particles in the nanosize could induce cleavage of caspase- 1 and secretion of IL-1 $\beta$ (25). Caspase- 1 activity of stratum corneum and serum IL-18 level were also increased in patients with Netherton syndrome, a disease characterized by chronic skin inflammation (26). In a tetanus toxoid-dependent experimental model using cocultures of monocytes and keratinocytes, others furthermore observed high levels of IL-1 $\beta$ when tetanus toxoid and keratinocytes were present, pointing to the latter cells to secrete caspase-1 and a source for IL-1 $\beta$ (27) (Figure 2).

In skin injury models relevant to the development of cancer, irradiation with a physiological dose of UVB induced secretion of pro-IL- $1 \alpha$ and of mature and active IL-1 $\beta$ and IL-18 in a caspase-1 dependent fashion $(28,29)$. Other studies of UVB overexposure in sunburned skin demonstrated activated inflammasomes (30) and UV light exposure stimulated bFGF and HMGB1 release by keratinocytes as well $(31,32)$. In an allergic skin disease model, mite allergen Dermatophagoides pteronyssinus 1 (Der p1, a major allergen of house dust mite), is recognized as a danger signal, activated caspase- 1 and induced release of IL- $1 \beta$ and IL-18 from keratinocytes which was dependent on the cysteine protease activity. Moreover, Der $\mathrm{p} 1$ stimulated assembly of the inflammasome by recruiting ASC, caspase-1, and NLRP3 to the perinuclear region (33).

The data reviewed in this section demonstrate that keratinocytes are a potent source of cytokines and alarmins upon contact with a broad spectrum of activators. It is clear that keratinocytes do not only have a passive role as target cells in the process of inflammation, but also act as stimulators of the initiation and maintenance of local immune reactions. 


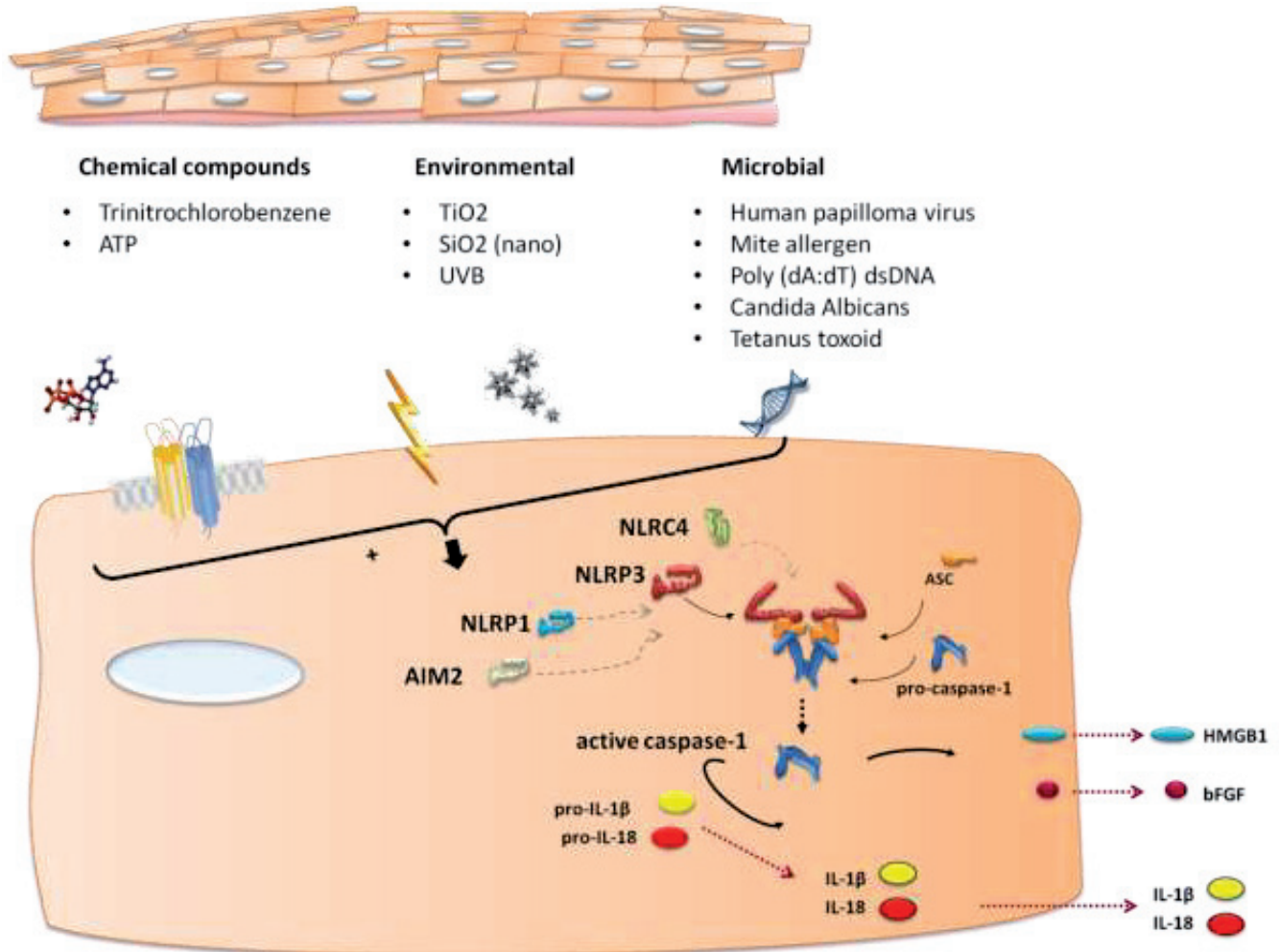

Figure 2: Schematic representation of stratified squamous epithelium of the skin and oral mucosa. Toxic molecules, environmental and cellular stressors as well as microbial antigens can individually activate one or more inflammasome subtypes, leading to caspase-1 activation and the release of $I L-1 \beta$, IL-18, bFGF and HMGB1.

\section{ORAL MUCOSA}

The oral mucosa is exposed to a high density and diversity of potential microbial pathogens such as gram-positive and gram-negative bacteria as well as fungi and others, and therefore has the important function to acts as a physical barrier and to respond to microbial growth and invasion. The inflammasomes, as intracellular immune receptors, are thus likely to be important mediators of the inflammatory response in gingival epithelial cells. In a recent study depletion of NLRP3 by siRNA abrogated the ability of ATP to induce IL- $1 \beta$ secretion in infected cells (34). ATP is sensed by purinergic receptors such as $\mathrm{P} 2 \mathrm{X}$, ligand-gated ion channel 4 (P2X4). Besides numerous reports on the role of P2X7 receptors in ATP-mediated inflammasome activation and mature IL- $1 \beta$ production in macrophages in vitro, recently in gingival epithelial cells that were stimulated with extracellular ATP, a role for P2X7 dependent-ROS production in the activation of the inflammasome was revealed (Figure 2) (35). Despite, its role in vivo has recently been questioned. Interestingly, in addition to the NLRP3 inflammasome, a different inflammasome containing NLRC4 appeared to function in the protection against infection with Candida albicans in the mucosal lining of the mouth and intestines rather than in immune cells (36). No further evidence can be found on how immune 
homeostasis via inflammasome signaling is maintained in this environment. These studies reveal the epithelial specific roles of the NLRP3 and NLRC4 inflammasome in innate immune response of the oral mucosa.

\section{GUT}

In organs where a variety of cell types come in intimate contact with commensals and potentially pathogenic microbes, such as the gut, the regulation and maintenance of normal intestinal mucosal barrier function is primordial for the host's survival and fitness. When cellular integrity and functioning of tight junctions between adjacent epithelial cells is disrupted barrier impairment is easily provided resulting in inflammation and the induction of tissue repair responses. The lack of control of this inflammatory condition is suggested to aggravate in the direction of detrimental chronic inflammation in the gut. Inflammasomedependent mediators such as IL-1 $\beta$, IL-18 and HMGB1 have been identified as potent promoters of intestinal pathology, which suggests that targeting these mediators may represent a useful therapeutic approach in bowel disease (IBD) (37).

Initially, observations suggested that induction of IL-1 $\beta$ mRNA in enterocytes was causally related to the subsequent inflammatory changes seen in a model of acute experimental colitis (38). It was proposed that colon epithelial cells were programmed to provide a set of signals for the activation of the mucosal inflammatory response in the earliest phases after microbial invasion $(39,40)$. Later, a few studies demonstrated that intestinal epithelial cells (IEC), continuously exposed to dietary molecules, microbial antigens and environmental influences, played a much more active role in the host immune and inflammatory response via the secretion of a variety of cytokines not limited to IL-1 $\beta$, but also IL-1 $\alpha$ and IL-8 (41-43). That same year for the first time, mouse IECs were proven to be the main producers of IL-18, formerly called interferon-gamma-inducing factor (44) under normal physiological conditions, suggesting that its constitutive expression in IECs may have an important role in the induction of mucosal immunity (45). Two years later, IL-18 was demonstrated to be localized and increasingly expressed in intestinal mucosal cells of patients with Crohn's disease (CD) $(46,47)$. Within this same period, post-translational activation of IL-18 by caspase-1 cleavage was identified to occur in response to viral and bacterial infections (48, 49). Specifically, a year before inflammasomes were characterized, cleavage of IL-18 in porcine intestinal mucosa by Salmonella choleraesuis was demonstrated, indicating that caspase-1 activation of IL-18 may be a key step in mucosal immune response to bacterial invasion (50). Expression of IL-18 in human gastric mucosal epithelial cells was also increased by Helicobacter pylori infection or by lactoferrin $(51,52)$. Recently, human IECs showed the ability to release of IL-18 upon salmonella treatment in a caspase- 1 dependent fashion (53) and release HMGB1 in their culture medium upon stimulation with LPS (54) and a mixture of TNF- $\alpha$, IL-1 $\beta$, and IFN- $\gamma$ (55).

Microbial activity is required to be constantly monitored in the epithelial lining of the gut. It has become evident that a range of inflammasome family members within different cell types (e.g. epithelial and hematopoietic cells) accomplish different, but often complementary functions, as watchful guardians eliciting mucosal immune responses when activated (56). The most intensively studied inflammasomes in the gut, the NLRP1, NLRP3, NLRP6 and 
NLRC4 inflammasomes, have been shown to regulate a number of common intestinal mucosal infections. Importantly, different enteric infections are sensed by and linked to different inflammasome functionalities. For instance, NLRP3 and NLRC4 activation in the intestinal epithelium is essential for regulation of permeability and epithelial regeneration through sensing of commensal microbes and has been shown to protect against mucosal pathogens $(57,58)$, however, excessive inflammasome activation within the lamina propria contributes to severe intestinal inflammation (59). Moreover, whereas the NLRP6 inflammasome subtype regulated colonic microbial ecology and risk for colitis (60), it was also shown to be involved in control of epithelial self-renewal and colorectal carcinogenesis upon injury (61). Additionally, recent findings suggest both hematopoietic- and nonhematopoietic-derived NLRP12 contributed to inflammation in an experimental colitis model, but the latter dominantly contributed to tumorigenesis. Herein, NLRP12 was profiled as an important add-on in the inflammasome repertoire and new player in colonic inflammation and tumorigenesis (62). Together, these studies reveal intensive and integrated signaling from multiple inflammasomes to regulate inflammation-induced IBD and colon cancer. In addition, unpublished data report the upregulation of most inflammasome sensor subtypes (NLRP1, NLRP3, NLRP12, NLRC4, AIM2, IFI16, MNDA and PYHIN1) in the colonic mucosa of active IBD patients, with the double stranded (ds)DNA responding PYHIN inflammasome subtypes (AIM2 and IFI16) showing the strongest increase. These data are accompanied with enhanced levels of IL-1 $\beta$ in primary IECs in culture following dsDNA exposure. Immunohistochemical data show, next to inflammatory cells, an epithelial presence of these inflammasome sensor subunits and some of their effector molecules (CASP1 and HMGB1) (unpublished data). Together, this indicates that a more profound focus on non-NLR signaling may be justified in IBD. The multiple activators of a broad spectrum of inflammasome subtypes implying caspase-1 activation and subsequent secretion of specific readouts in IECs are summarized in Figure 3. 


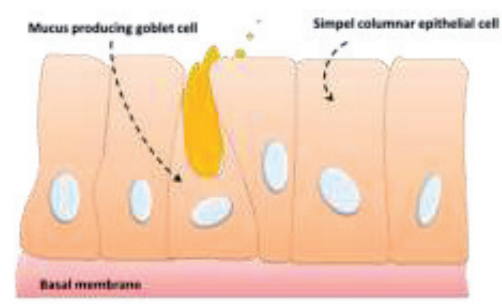

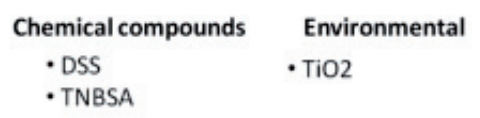

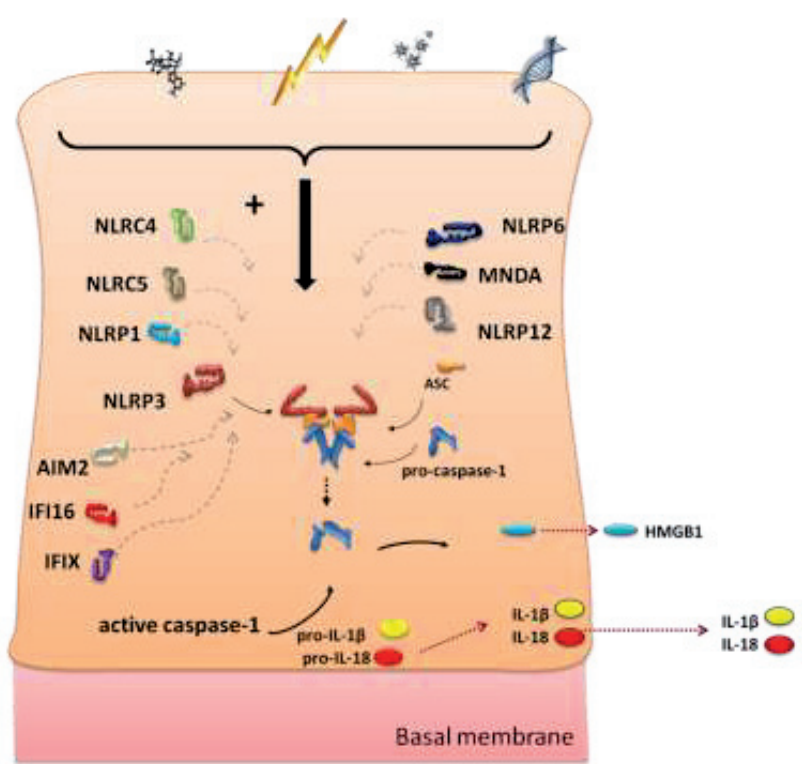

Figure 3: Schematic representations of simple columnar epithelial cells lining the digestive tract reflecting multiple inflammasomes that are described to be activated by different described agents AIM2, IFI16, MNDA and PYHIN1 are subtypes of the non-NLR inflammasome family.

Activation of intestinal inflammasomes in different lineages of cells regulates physiological reactions, and their hyper-activation or absence can lead to deleterious consequences such as inflammation or cancer progression as shown in different models (56). For instance, following tissue damage using the IEC cytotoxic agent dextran sodium sulphate (DSS), the NLRP3 inflammasome assembles, leading to the production of IL-18, which is then released at the mucosal sites (63). Defective NLRP3 inflammasome subtype activation was shown to protect against loss of epithelial integrity and mortality during DSS-induced experimental colitis (64), suggesting that genetic and environmental factors may activate the NLRP3 inflammasome (65). In addition, their absence rather than their over production could be considered deleterious, indicating a multifaceted regulatory role of NLRP3 in intestinal inflammation. Normand et al., on the other hand, demonstrated that NLRP6-deficient mice were highly susceptible to experimental colitis (61). Further, it was shown in humans with a leaky intestinal barrier (such as seen in IBD patients) that $\mathrm{TiO}_{2}$ microparticles were taken up by IEC, and could activate the inflammasome and induce IL-1 $\beta$ and IL-18 secretion in the mucosa of Crohn's disease patients, representing a possible mode of aggravation of inflammation in susceptible individuals (66). Others have shown that 2,4,6-trinitrobenzene sulfonic acid (TNBSA) was unable to induce significant colitis in IL-18 deficient mice and 
that administration of an IL-18 neutralizing antibody resulted in a dramatic attenuation of mucosal inflammation. The proposed function for the NLR and non-NLR inflammasomes is to regulate secretion of IL-18 that stimulates epithelial cell barrier function and regeneration, whereas in hematopoietic cells, inflammasome activation would have a proinflammatory effect $(60,67)$. This suggests that signals produced by the IECs may play an important role in inducing the early host inflammatory response to infection and raises the possibility that interventions that directly target production of inflammatory cytokines by IECs might alter the course of disease. When comparing results of studies by different groups, one should take into consideration that many of the observed effects may be explained by defective inflammasome regulation of the composition of the microflora coupled with differences in native microflora in different facilities (56).

Targeting mediator release that is associated with mucosal inflammasome activation in the gut could lead to a better understanding to which pathological aspects of inflammation and subsequent increases in permeability contribute to the development of IBD.

\section{LUNG}

Barrier epithelia, such as the airway epithelial cells lining the respiratory tract, fulfill multiple functions essential for tissue homeostasis. They are, because of the immense surface area that is in intimate contact with the environment, a primary target of attack by micro-organisms and potentially harmful factors during every single breath. A vicious cycle of exaggerated responses to chronic stimuli or aberrant responses to rather innocent agents may result in chronic inflammation with permanent structural changes in barrier properties, including smooth muscle hyperplasia, airway remodeling and fibrosis. The importance of engagement of pattern recognition receptors (PPRs) and their activation is demonstrated via experimental studies in knockout mice. These resulted in evidence suggesting a deleterious role for excessive production of the inflammasome-dependent proinflammatory cytokines and danger signals IL-1 $\beta$, IL-18, HMGB1 and the growth factor bFGF which possess multiple pathogenic properties that could be further enhanced during episodes of disease exacerbations (68-72). Because the localization and the impact of inflammasome sensor activation in airway epithelial cells associated with pulmonary inflammation has yet to be revealed, the activation of different inflammasomes in lung epithelium in response to triggers relevant to the main chronic inflammatory diseases; asthma, COPD, pulmonary fibrosis and pneumoconiosis, mainly in in vitro settings is summarized in this section.

Historically, with respect to evidence of inflammasome activation or mediator release from lung epithelium one has to go back more than a decade prior to the first characterization of the inflammasome. Immunoreactive IL- $1 \beta$ was shown to be released from bronchial epithelial cells exposed to (toluene di-) isocyanates (73) or nitrogen dioxide $(74,75)$, and in tracheal biopsy material from individuals exposed to endotoxin-contaminated grain dust. A few years later, in 1996 Hastie et al. showed low but significantly higher amounts (2-fold) of IL-1 $\beta$ released from bronchial epithelial cells from allergic compared to non-allergic individuals following segmental challenge with ragweed (76). Moreover, in experiments with exposure to another allergen, Der $\mathrm{p} 1$, cultured human airway epithelial cells were shown to release IL-1 $\beta$ (77). In 1998, the IL-1 $\beta$ release for the first time was linked to ICE expression in alveolar 
epithelial cells upon respiratory syncytial virus (RSV) infection. Interestingly this study showed that this occurred in the absence of apoptosis (45), which could imply that epithelial cell death occurred through what is now known as pyroptosis (Figure 4).

Different animal models of pulmonary fibrosis have been developed to investigate its pathogenesis and potential therapies for idiopathic pulmonary fibrosis (IPF). The most common is the bleomycin model in rodents (mouse, rat and hamster) (78). In 2001, caspase-1 mRNA expression was shown to be elevated in mice treated with bleomycin, and bronchiolar and alveolar epithelial cells, as well as myeloid cells showed increased caspase-1 immunoreactivity in both nucleus and cytoplasm (79). A decade later, it was suggested that inflammasome signaling in airway epithelial cells may play an important role in the pathogenesis of diseases like COPD, as compounds such as LPS and CpG were found to induce the releases of IL-1 $\beta$ from human bronchial epithelial cells (80). Next to these environmental triggers, mechanical stretch was shown that same year to induce enhanced IL$1 \beta$ levels in the supernatants of alveolar epithelial cells (81). In recent years the panel of mediators able to activate the inflammasome-dependent caspase-1 activity and IL-1 $\beta$ release from (primary) lung epithelial cells has expanded rapidly to include Pseudomonas aeroginosa, Simvastatin (82), Influenza A (16, 83, 84), RSV (85) and rhinovirus (86). The list of activators seems to be unlimited. The membrane attack complex of complement, apart from its classical role of lysing cells, can also trigger a range of non-lethal effects on cells, including driving inflammation. Recent findings demonstrated that sublytic attack by the membrane attack complex of complement, leads to caspase-1 activation as well as IL-1 $\beta$ secretion in primary human lung epithelial cells (87). Another class of inflammasome activators in lung epithelial cells includes noxious inhaled particles. A panel of inflammasome-dependent mediators were shown to be released by bronchial epithelial cells following crystalline silica exposure (88) and Tran et al. demonstrated induction of IL-1 $\beta$ and NLRP3 protein by the proinflammatory stimulus LPS and the combination of IFN- $\gamma$ with LPS in primary cell cultures of NHBE cells (89). Hirota and his colleagues characterized airway epithelial NLRP3 inflammasome-mediated immune responses to urban particulate matter exposure and found significant increases in airway epithelial NLRP3 inflammasomemediated production of IL-1 $\beta$ in vitro, results that were corroborated in vivo (90).

For interleukin-18, a profilic cytokine involved in many immune responses already issued, literature research revealed its immunoreactivity in airway epithelial cells was first investigated during early stages of host defense within the bronchial epithelium of biopsies obtained from control subjects and patients with sarcoidosis or asthma (91). Later, Western blot analysis showed that the $18.3 \mathrm{kDa}$ mature form of IL-18 appeared in whole cell lysate of Mycobacterium tuberculosis -stimulated alveolar type II cells, whereas both non-stimulated and Mycobacterium tuberculosis-stimulated alveolar type II cells contained abundant $24 \mathrm{kDa}$ pro-IL-18. These results indicated that Mycobacterium tuberculosis up-regulates IL-18 expression at both transcriptional and posttranscriptional levels (92), implying the involvement of caspase-1 enzymatic activity and therefore inflammasome activation. Piper et al furthermore found that IL-18 was released from Rhinovirus-infected lung epithelia. The release was not associated with cell death but dependent on caspase-1 catalytic activity (86). 
The endogenous danger protein HMGB1 was shown to be released from A549 cells infected with virulent Legionella in association with caspase-1 activity (93). HMGB1 levels were furthermore found to be elevated in cell supernatant from rat alveolar type II cell monolayers that underwent scratch wounding (94). Moreover, mechanical stretch significantly increased HMGB1 protein expression in A549 cells (81). These results are important in the context of injury, since epithelial crosstalk to neighboring cells is important for normal as well aberrant repair, such as in the case of fibrosis. HMGB1 was included in the panel of inflammasomedependent mediators that were released following crystalline silica exposure of bronchial epithelial cells (88).

Epithelial cells not only express and secrete cytokines and alarmins upon exposure to endogenous or exogenous inflammasome activators but also bFGF. The FGFs are involved in morphogenesis, wound repair, inflammation, angiogenesis, and tumour growth and invasion, and require the glycosaminoglycan (GAG) side chains of heparin sulphate proteoglycans for high affinity binding to their specific receptors (95). Late $20^{\text {th }}$ century, bronchial epithelial cells were shown to secrete bFGF which positively impacted myofibroblast proliferation in an animal model of asthma. A role for epithelial cells in the expression and release of bFGF from heparan sulphate binding sites in bronchial asthma was defined a couple years later (96). Treatment of human fibroblasts with caspase- 1 inhibitors significantly reduced the amount of secreted bFGF (4). The lung epithelium is a major source of bFGF as shown by rhinovirusinduced bFGF release in a model that mimics features of airway remodeling (68). We furthermore $\mathrm{s}$ showed that crystalline silica exposure of bronchial epithelial cells caused bFGF release (97) which was inflammasome- and particle uptake- dependent (88). Importantly, our studies showed that the panel of silica-induced NLRP3 inflammasomedependent mediators released from airway epithelium leads to fibroblast proliferation, a characteristic of multiple lung diseases. These findings are paralleled by work of Hussain S. et al., in which it is evidenced that multi-walled carbon nanotubes induce a NLRP3 inflammasome-dependent, but TGF- $\beta$ independent pro-fibrotic response in human bronchial epithelial cells (98). In contrast, there are publications that demonstrate that lung epithelial cells are not able to secrete IL-1 $\beta$ upon exposure to different micro-organisms and particulates among others $(99,100)$. This section however summarizes a vast body of evidence that lung epithelium participates in early first line immune defenses via activation of the inflammasome. The expression of IL-18, IL-1 $\beta$, bFGF as well as HMGB1 by these cells demonstrates its participation in the initial response to encounters with foreign molecules. The described mediators may play a prominent role in the cascade of subsequent steps of the immune response in an autocrine, paracrine as well as chemotactic manner. 


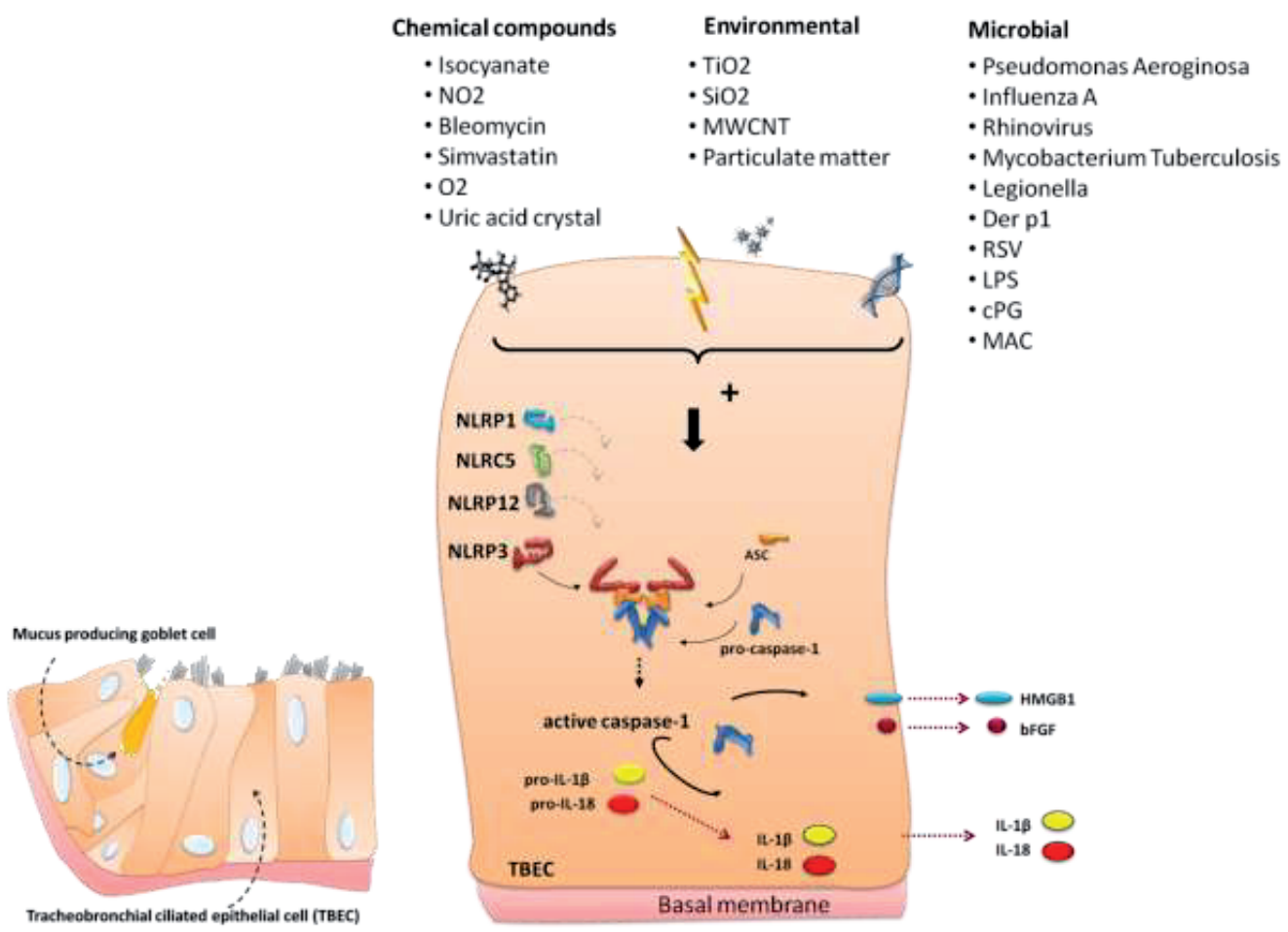

Figure 4: Schematic representation of pseudostratified columnar epithelium of the lung indicating a variety of environmental and microbial molecules that is able to activate the inflammasome with a subsequent release of cytokines, alarmins and growth factors.

To date, only a subset of inflammasomes has been described in lung epithelial cells so far. NLRP1 was reported to contribute to the immune response in lung epithelial cells and alveolar macrophages (22). NLRP3 inflammasome presence and activation in lung epithelial cells was demonstrated as well $(89,90)$. Of most caspase-1 activating inflammasomes that have been studied well, NLRP12 is a unique NLR that has been shown to attenuate inflammatory pathways in biochemical assays and mediates the lymph node homing of activated skin dendritic cells in contact hypersensitivity responses. Although its expression was shown in lung cells, the overall development of allergic airway disease and airway function was not significantly altered by overall NLRP12 deficiency. This suggests that NLRP12 does not play a vital role in regulating airway inflammation in this model (101). Upon rhinovirus pathogenesis, the contribution of NLRP3 and NLRC5 inflammasomes and IL-1 $\beta$ secretion in rhinovirus pathogenesis was investigated and revealed that both inflammasomes act in a cooperative manner during the assembly by sensing intracellular $\mathrm{Ca}^{2+}$ fluxes and triggering IL-1 $\beta$ secretion in primary human bronchial epithelial cells (102). Additionally, the importance of inflammasome signaling in animal models representing a cadre of lung diseases such as asthma, COPD, acute lung injury as well as fibrosis and pneumoconiosis among others has been shown by many groups (103-111) and was recently 
reviewed by Brusselle GG et al. (112). However no focus on the epithelium is present in any model.

This section evidences that the surface epithelium of the conducting airways can be considered a constitutive primary participant in innate immunity with strong evidence that epithelial dysfunction is involved in the development of inflammatory disorders of the lung, and could be a plausible target for therapeutic interventions. Often though, as indicated in the preceding paragraph the importance of inflammasome activation in the epithelium in animal models is not primarily approached. Therefore conditional knockout models or epithelial specific transgenic animal studies will be a necessity.

\section{UROGENITAL}

With respect to cells lining the urogenital tract, evidence suggests that inflammasomes, next to other PPRs, have important roles in associated diseases through regulation of inflammatory and tissue-repair responses to infection and injury (113). First, on the subject of human kidney diseases such as Wegener's granulomatosis and in experimental models of glomerulonephritis, glomerular as well as tubular epithelial cells have been shown to synthesize and release IL-1 $\beta$, constitutively (114-116). In a recent study that analyzed the processing of caspase-1, IL-1 $\beta$, and IL-18 after unilateral ureteral obstruction (UUO) in mice reflecting chronic kidney disease, it was shown that NLRP3 has a biological function in both hematopoietic and renal epithelial compartments during renal injury. Additionally, in models of ischemic tubular necrosis and obstruction-induced epithelial-mesenchymal transition, an important role for caspase-1 and IL-18 has been demonstrated under hypoxic conditions and in the absence of vascular effects (117-119). Other cells lining epithelial tracts in contact with the environment conveying inflammasomes are prostate epithelial cells expressing AIM2 with increased caspase- 1 activity in an experimental model of benign prostate hyperplasia (BPH), and human cervical epithelial cells expressing AIM2 and IFI16 inflammasomes following Chlamydia trachomatis and Herpes simplex virus 2, respectively (120-122). Although the amount of literature on inflammasome activation in these organs is relatively scarce, other studies demonstrate a pivotal role of the presence and activation of various inflammasomes in the epithelium of urogenital organs exposed to the environment (123-125) (Figure 5). 


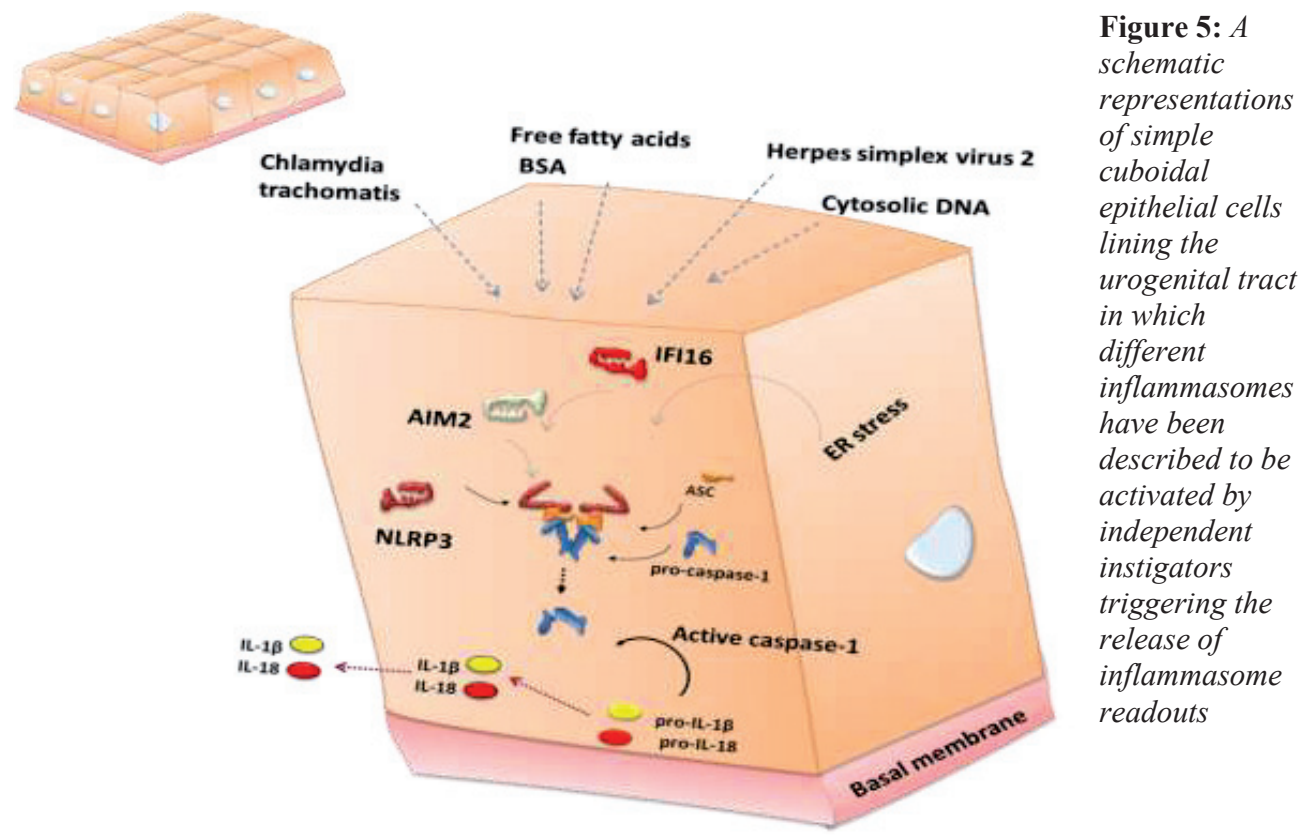

\section{CONCLUSION}

Epithelial cells form an interface between the body and the environment. Therefore, they are important guardians for the detection of danger signals and the consecutive initiation of an inflammatory response. As presented in this review, each organ and cell type expresses different sensor subtypes with discrepancy in the release of various mediators. It should be emphasized that it is very possible that manifold inflammasomes are important in multiple epithelial cell types and become activated to either overcome detrimental signaling or to cooperate in a constructive fashion combating the disease. Likewise, with respect to relatively lower concentrations of cytokines released from epithelial cells versus the myeloid compartment, it could be considered that first line barrier epithelial cells, in contact with many potential danger signals, preferably should not produce high amounts of these very potent inflammatory cytokines and alarmins as it would be harmful for the microenvironment to have a constant "high-alarm situation". Additionally, many more epithelial cells are present in these organs as opposed to for instance macrophages; therefore activated epithelial cells may relay equally large and biologically significant immune signals that build up the important contribution in global inflammasome activation at organ level.

This review demonstrates that inflammasome activation and subsequent secretion of 'alarming' proteins is not restricted to macrophages, indicating that epithelial cells should be considered as highly important cells in innate immune signaling. In future research, epithelial-specific conditional knockout models and transgenic animal studies will be a necessary approach to determine this important contribution more profoundly. 


\section{REFERENCES}

1. Medzhitov R. Origin and physiological roles of inflammation. Nature. 2008 Jul 24;454(7203):428-35.

2. Dinarello CA. Interleukin-1. Rev Infect Dis. 1984 Jan-Feb;6(1):51-95.

3. Gracie JA, Robertson SE, McInnes IB. Interleukin-18. J Leukoc Biol. 2003 Feb;73(2):213-24.

4. Keller M, Ruegg A, Werner S, Beer HD. Active caspase-1 is a regulator of unconventional protein secretion. Cell. 2008 Mar 7;132(5):818-31.

5. Martinon F, Burns K, Tschopp J. The inflammasome: a molecular platform triggering activation of inflammatory caspases and processing of proIL-beta. Mol Cell. 2002 Aug;10(2):417-26.

6. Schattgen SA, Fitzgerald KA. The PYHIN protein family as mediators of host defenses. Immunol Rev. 2011 Sep;243(1):109-18.

7. Keyel PA. How is inflammation initiated? Individual influences of IL-1, IL-18 and HMGB1. Cytokine. 2014 Apr 15.

8. Lamkanfi M, Dixit VM. Mechanisms and Functions of Inflammasomes. Cell. 2014 May $22 ; 157(5): 1013-22$.

9. Petrilli V, Papin S, Tschopp J. The inflammasome. Curr Biol. 2005 Aug 9;15(15):R581.

10. Vande Walle L, Kanneganti TD, Lamkanfi M. HMGB1 release by inflammasomes. Virulence. 2011 Mar-Apr;2(2):162-5.

11. Miao EA, Rajan JV, Aderem A. Caspase-1-induced pyroptotic cell death. Immunol Rev. 2011 Sep;243(1):206-14.

12. Kumar H, Kawai T, Akira S. Pathogen recognition by the innate immune system. Int Rev Immunol. 2011 Feb;30(1):16-34.

13. Liu D, Rhebergen AM, Eisenbarth SC. Licensing Adaptive Immunity by NOD-Like Receptors. Front Immunol. 2013;4:486.

14. Matzinger P. Friendly and dangerous signals: is the tissue in control? Nat Immunol. 2007 Jan;8(1):11-3.

15. Tsuji NM, Kosaka A. Oral tolerance: intestinal homeostasis and antigen-specific regulatory $\mathrm{T}$ cells. Trends Immunol. 2008 Nov;29(11):532-40.

16. Watanabe H, Gaide O, Petrilli V, Martinon F, Contassot E, Roques S, et al. Activation of the IL-1beta-processing inflammasome is involved in contact hypersensitivity. J Invest Dermatol. 2007 Aug;127(8):1956-63.

17. Petrilli V, Dostert C, Muruve DA, Tschopp J. The inflammasome: a danger sensing complex triggering innate immunity. Curr Opin Immunol. 2007 Dec;19(6):615-22.

18. Yazdi AS, Drexler SK, Tschopp J. The role of the inflammasome in nonmyeloid cells. J Clin Immunol. 2010 Sep;30(5):623-7. 
19. Pasparakis M, Haase I, Nestle FO. Mechanisms regulating skin immunity and inflammation. Nat Rev Immunol. 2014 May;14(5):289-301.

20. Cooper KD, Hammerberg C, Baadsgaard O, Elder JT, Chan LS, Taylor RS, et al. Interleukin-1 in human skin: dysregulation in psoriasis. J Invest Dermatol. 1990 Nov;95(5):24S-6S.

21. Zepter K, Haffner A, Soohoo LF, De Luca D, Tang HP, Fisher P, et al. Induction of biologically active IL-1 beta-converting enzyme and mature IL-1 beta in human keratinocytes by inflammatory and immunologic stimuli. J Immunol. 1997 Dec 15;159(12):6203-8.

22. Kummer JA, Broekhuizen R, Everett H, Agostini L, Kuijk L, Martinon F, et al. Inflammasome components NALP 1 and 3 show distinct but separate expression profiles in human tissues suggesting a site-specific role in the inflammatory response. J Histochem Cytochem. 2007 May;55(5):443-52.

23. Kopfnagel V, Wittmann M, Werfel T. Human keratinocytes express AIM2 and respond to dsDNA with IL-1beta secretion. Exp Dermatol. 2011 Dec;20(12):1027-9.

24. Reinholz M, Kawakami Y, Salzer S, Kreuter A, Dombrowski Y, Koglin S, et al. HPV16 activates the AIM2 inflammasome in keratinocytes. Arch Dermatol Res. 2013 Oct;305(8):72332 .

25. Yazdi AS, Guarda G, Riteau N, Drexler SK, Tardivel A, Couillin I, et al. Nanoparticles activate the NLR pyrin domain containing 3 (Nlrp3) inflammasome and cause pulmonary inflammation through release of IL-1alpha and IL-1beta. Proc Natl Acad Sci U S A. 2010 Nov 9;107(45):19449-54.

26. Hosomi N, Fukai K, Nakanishi T, Funaki S, Ishii M. Caspase-1 activity of stratum corneum and serum interleukin-18 level are increased in patients with Netherton syndrome. Br J Dermatol. 2008 Sep;159(3):744-6.

27. Renne J, Schafer V, Werfel T, Wittmann M. Interleukin-1 from epithelial cells fosters $\mathrm{T}$ celldependent skin inflammation. Br J Dermatol. 2010 Jun;162(6):1198-205.

28. Feldmeyer L, Keller M, Niklaus G, Hohl D, Werner S, Beer HD. The inflammasome mediates UVB-induced activation and secretion of interleukin-1beta by keratinocytes. Curr Biol. 2007 Jul $3 ; 17(13): 1140-5$.

29. Feldmeyer L, Werner S, French LE, Beer HD. Interleukin-1, inflammasomes and the skin. Eur J Cell Biol. 2010 Sep;89(9):638-44.

30. Faustin B, Reed JC. Sunburned skin activates inflammasomes. Trends Cell Biol. 2008 Jan;18(1):4-8.

31. Dong L, Li Y, Cao J, Liu F, Pier E, Chen J, et al. FGF2 regulates melanocytes viability through the STAT3-transactivated PAX3 transcription. Cell Death Differ. 2012 Apr;19(4):616-22.

32. Johnson KE, Wulff BC, Oberyszyn TM, Wilgus TA. Ultraviolet light exposure stimulates HMGB1 release by keratinocytes. Arch Dermatol Res. 2013 Nov;305(9):805-15.

33. Dai X, Sayama K, Tohyama M, Shirakata Y, Hanakawa Y, Tokumaru S, et al. Mite allergen is a danger signal for the skin via activation of inflammasome in keratinocytes. J Allergy Clin Immunol. 2011 Mar;127(3):806-14 e1-4. 
34. Yilmaz O, Sater AA, Yao L, Koutouzis T, Pettengill M, Ojcius DM. ATP-dependent activation of an inflammasome in primary gingival epithelial cells infected by Porphyromonas gingivalis. Cell Microbiol. 2010 Feb;12(2):188-98.

35. Hung SC, Choi CH, Said-Sadier N, Johnson L, Atanasova KR, Sellami H, et al. P2X4 assembles with P2X7 and pannexin-1 in gingival epithelial cells and modulates ATP-induced reactive oxygen species production and inflammasome activation. PLoS One. 2013;8(7):e70210.

36. Tomalka J, Ganesan S, Azodi E, Patel K, Majmudar P, Hall BA, et al. A novel role for the NLRC4 inflammasome in mucosal defenses against the fungal pathogen Candida albicans. PLoS Pathog. 2011 Dec;7(12):e1002379.

37. Aguilera M, Darby T, Melgar S. The complex role of inflammasomes in the pathogenesis of Inflammatory Bowel Diseases - Lessons learned from experimental models. Cytokine Growth Factor Rev. 2014 Apr 18.

38. Radema SA, van Deventer SJ, Cerami A. Interleukin 1 beta is expressed predominantly by enterocytes in experimental colitis. Gastroenterology. 1991 May;100(5 Pt 1):1180-6.

39. Jung HC, Eckmann L, Yang SK, Panja A, Fierer J, Morzycka-Wroblewska E, et al. A distinct array of proinflammatory cytokines is expressed in human colon epithelial cells in response to bacterial invasion. J Clin Invest. 1995 Jan;95(1):55-65.

40. Stadnyk AW, Kearsey JA. Pattern of proinflammatory cytokine mRNA expression during Trichinella spiralis infection of the rat. Infect Immun. 1996 Dec;64(12):5138-43.

41. Kagnoff MF. Mucosal immunology: new frontiers. Immunol Today. 1996 Feb;17(2):57-9.

42. Seydel KB, Li E, Swanson PE, Stanley SL, Jr. Human intestinal epithelial cells produce proinflammatory cytokines in response to infection in a SCID mouse-human intestinal xenograft model of amebiasis. Infect Immun. 1997 May;65(5):1631-9.

43. Vallette G, Jarry A, Lemarre P, Branka JE, Laboisse CL. NO-dependent and NO-independent IL-1 production by a human colonic epithelial cell line under inflammatory stress. Br J Pharmacol. 1997 May;121(2):187-92.

44. Nakamura K, Okamura H, Wada M, Nagata K, Tamura T. Endotoxin-induced serum factor that stimulates gamma interferon production. Infect Immun. 1989 Feb;57(2):590-5.

45. Takeuchi M, Nishizaki Y, Sano O, Ohta T, Ikeda M, Kurimoto M. Immunohistochemical and immuno-electron-microscopic detection of interferon-gamma-inducing factor ("interleukin-18") in mouse intestinal epithelial cells. Cell Tissue Res. 1997 Sep;289(3):499-503.

46. Pizarro TT, Michie MH, Bentz M, Woraratanadharm J, Smith MF, Jr., Foley E, et al. IL-18, a novel immunoregulatory cytokine, is up-regulated in Crohn's disease: expression and localization in intestinal mucosal cells. J Immunol. 1999 Jun 1;162(11):6829-35.

47. Monteleone G, Trapasso F, Parrello T, Biancone L, Stella A, Iuliano R, et al. Bioactive IL-18 expression is up-regulated in Crohn's disease. J Immunol. 1999 Jul 1;163(1):143-7.

48. Ghayur T, Banerjee S, Hugunin M, Butler D, Herzog L, Carter A, et al. Caspase-1 processes IFN-gamma-inducing factor and regulates LPS-induced IFN-gamma production. Nature. 1997 Apr 10;386(6625):619-23. 
49. Gu Y, Kuida K, Tsutsui H, Ku G, Hsiao K, Fleming MA, et al. Activation of interferon-gamma inducing factor mediated by interleukin-1beta converting enzyme. Science. 1997 Jan 10;275(5297):206-9.

50. Foss DL, Zilliox MJ, Murtaugh MP. Bacterially induced activation of interleukin-18 in porcine intestinal mucosa. Vet Immunol Immunopathol. 2001 Feb 10;78(3-4):263-77.

51. Shimada M, Ando T, Peek RM, Watanabe O, Ishiguro K, Maeda O, et al. Helicobacter pylori infection upregulates interleukin-18 production from gastric epithelial cells. Eur J Gastroenterol Hepatol. 2008 Dec;20(12):1144-50.

52. Yang JC, Yang HC, Shun CT, Wang TH, Chien CT, Kao JY. Catechins and Sialic Acid Attenuate Helicobacter pylori-Triggered Epithelial Caspase-1 Activity and Eradicate Helicobacter pylori Infection. Evid Based Complement Alternat Med. 2013;2013:248585.

53. Knodler LA, Vallance BA, Celli J, Winfree S, Hansen B, Montero M, et al. Dissemination of invasive Salmonella via bacterial-induced extrusion of mucosal epithelia. Proc Natl Acad Sci U S A. 2010 Oct 12;107(41):17733-8.

54. Vitali R, Palone F, Cucchiara S, Negroni A, Cavone L, Costanzo M, et al. Dipotassium Glycyrrhizate Inhibits HMGB1-Dependent Inflammation and Ameliorates Colitis in Mice. PLoS One. 2013;8(6):e66527.

55. Liu S, Stolz DB, Sappington PL, Macias CA, Killeen ME, Tenhunen JJ, et al. HMGB1 is secreted by immunostimulated enterocytes and contributes to cytomix-induced hyperpermeability of Caco-2 monolayers. Am J Physiol Cell Physiol. 2006 Apr;290(4):C990-9.

56. Elinav E, Henao-Mejia J, Flavell RA. Integrative inflammasome activity in the regulation of intestinal mucosal immune responses. Mucosal Immunol. 2013 Jan;6(1):4-13.

57. Song-Zhao GX, Srinivasan N, Pott J, Baban D, Frankel G, Maloy KJ. Nlrp3 activation in the intestinal epithelium protects against a mucosal pathogen. Mucosal Immunol. 2014 Jul;7(4):763-74.

58. Nordlander S, Pott J, Maloy KJ. NLRC4 expression in intestinal epithelial cells mediates protection against an enteric pathogen. Mucosal Immunol. 2014 Jul;7(4):775-85.

59. Lissner D, Siegmund B. The multifaceted role of the inflammasome in inflammatory bowel diseases. ScientificWorldJournal. 2011;11:1536-47.

60. Elinav E, Strowig T, Kau AL, Henao-Mejia J, Thaiss CA, Booth CJ, et al. NLRP6 inflammasome regulates colonic microbial ecology and risk for colitis. Cell. 2011 May 27;145(5):745-57.

61. Normand S, Delanoye-Crespin A, Bressenot A, Huot L, Grandjean T, Peyrin-Biroulet L, et al. Nod-like receptor pyrin domain-containing protein 6 (NLRP6) controls epithelial self-renewal and colorectal carcinogenesis upon injury. Proc Natl Acad Sci U S A. 2011 Jun 7;108(23):96016.

62. Allen IC, Wilson JE, Schneider M, Lich JD, Roberts RA, Arthur JC, et al. NLRP12 suppresses colon inflammation and tumorigenesis through the negative regulation of noncanonical NFkappaB signaling. Immunity. 2012 May 25;36(5):742-54. 
63. Saleh M, Trinchieri G. Innate immune mechanisms of colitis and colitis-associated colorectal cancer. Nat Rev Immunol. 2011 Jan;11(1):9-20.

64. Zaki MH, Boyd KL, Vogel P, Kastan MB, Lamkanfi M, Kanneganti TD. The NLRP3 inflammasome protects against loss of epithelial integrity and mortality during experimental colitis. Immunity. 2010 Mar 26;32(3):379-91.

65. Bauer C, Duewell P, Mayer C, Lehr HA, Fitzgerald KA, Dauer M, et al. Colitis induced in mice with dextran sulfate sodium (DSS) is mediated by the NLRP3 inflammasome. Gut. 2010 Sep;59(9):1192-9.

66. Becker HM, Bertschinger MM, Rogler G. Microparticles and their impact on intestinal immunity. Dig Dis. 2012;30 Suppl 3:47-54.

67. Siegmund B. Interleukin-18 in intestinal inflammation: friend and foe? Immunity. 2010 Mar 26;32(3):300-2.

68. Skevaki CL, Psarras S, Volonaki E, Pratsinis H, Spyridaki IS, Gaga M, et al. Rhinovirusinduced basic fibroblast growth factor release mediates airway remodeling features. Clin Transl Allergy. 2012;2(1):14.

69. Liou TG, Adler FR, Keogh RH, Li Y, Jensen JL, Walsh W, et al. Sputum biomarkers and the prediction of clinical outcomes in patients with cystic fibrosis. PLoS One. 2012;7(8):e42748.

70. Triantafilou K, Triantafilou M. Ion flux in the lung: virus-induced inflammasome activation. Trends Microbiol. 2014 Jun 28.

71. Chaput C, Sander LE, Suttorp N, Opitz B. NOD-Like Receptors in Lung Diseases. Front Immunol. 2013;4:393.

72. Vanden Berghe T, Demon D, Bogaert P, Vandendriessche B, Goethals A, Depuydt B, et al. Simultaneous targeting of IL-1 and IL-18 is required for protection against inflammatory and septic shock. Am J Respir Crit Care Med. 2014 Feb 1;189(3):282-91.

73. Mattoli S, Miante S, Calabro F, Mezzetti M, Fasoli A, Allegra L. Bronchial epithelial cells exposed to isocyanates potentiate activation and proliferation of T-cells. Am J Physiol. 1990 Oct;259(4 Pt 1):L320-7.

74. Devalia JL, Campbell AM, Sapsford RJ, Rusznak C, Quint D, Godard P, et al. Effect of nitrogen dioxide on synthesis of inflammatory cytokines expressed by human bronchial epithelial cells in vitro. Am J Respir Cell Mol Biol. 1993 Sep;9(3):271-8.

75. Devalia JL, Sapsford RJ, Cundell DR, Rusznak C, Campbell AM, Davies RJ. Human bronchial epithelial cell dysfunction following in vitro exposure to nitrogen dioxide. Eur Respir J. 1993 Oct;6(9):1308-16.

76. Hastie AT, Everts KB, Cho SK, Zangrilli J, Shaver JR, Pollice MB, et al. IL-1 beta release from cultured bronchial epithelial cells and bronchoalveolar lavage cells from allergic and normal humans following segmental challenge with ragweed. Cytokine. 1996 Sep;8(9):730-8.

77. Rusznak C, Sapsford RJ, Devalia JL, Shah SS, Hewitt EL, Lamont AG, et al. Interaction of cigarette smoke and house dust mite allergens on inflammatory mediator release from primary cultures of human bronchial epithelial cells. Clin Exp Allergy. 2001 Feb;31(2):226-38. 
78. Moeller A, Ask K, Warburton D, Gauldie J, Kolb M. The bleomycin animal model: a useful tool to investigate treatment options for idiopathic pulmonary fibrosis? Int J Biochem Cell Biol. 2008;40(3):362-82.

79. Kuwano K, Kunitake R, Maeyama T, Hagimoto N, Kawasaki M, Matsuba T, et al. Attenuation of bleomycin-induced pneumopathy in mice by a caspase inhibitor. Am J Physiol Lung Cell Mol Physiol. 2001 Feb;280(2):L316-25.

80. Mortaz E, Henricks PA, Kraneveld AD, Givi ME, Garssen J, Folkerts G. Cigarette smoke induces the release of CXCL-8 from human bronchial epithelial cells via TLRs and induction of the inflammasome. Biochim Biophys Acta. 2011 Sep;1812(9):1104-10.

81. Ding N, Wang F, Han Y, Xiao H, Xu L, She S. Mitogen-activated protein kinase kinase 6 mediates mechanical stretch-induced high-mobility group box 1 protein expression in pulmonary alveolar epithelial cells. J Trauma Acute Care Surg. 2012 Jan;72(1):162-8.

82. Zeki AA, Thai P, Kenyon NJ, Wu R. Differential effects of simvastatin on IL-13-induced cytokine gene expression in primary mouse tracheal epithelial cells. Respir Res. 2012;13:38.

83. Bauer RN, Brighton LE, Mueller L, Xiang Z, Rager JE, Fry RC, et al. Influenza enhances caspase-1 in bronchial epithelial cells from asthmatic volunteers and is associated with pathogenesis. J Allergy Clin Immunol. 2012 Oct;130(4):958-67 e14.

84. Pothlichet J, Meunier I, Davis BK, Ting JP, Skamene E, von Messling V, et al. Type I IFN triggers RIG-I/TLR3/NLRP3-dependent inflammasome activation in influenza A virus infected cells. PLoS Pathog. 2013;9(4):e1003256.

85. Triantafilou K, Kar S, Vakakis E, Kotecha S, Triantafilou M. Human respiratory syncytial virus viroporin SH: a viral recognition pathway used by the host to signal inflammasome activation. Thorax. 2013 Jan;68(1):66-75.

86. Piper SC, Ferguson J, Kay L, Parker LC, Sabroe I, Sleeman MA, et al. The role of interleukin-1 and interleukin-18 in pro-inflammatory and anti-viral responses to rhinovirus in primary bronchial epithelial cells. PLoS One. 2013;8(5):e63365.

87. Triantafilou K, Hughes TR, Triantafilou M, Morgan BP. The complement membrane attack complex triggers intracellular Ca2+ fluxes leading to NLRP3 inflammasome activation. J Cell Sci. 2013 Jul 1;126(Pt 13):2903-13.

88. Peeters PM, Perkins TN, Wouters EF, Mossman BT, Reynaert NL. Silica induces NLRP3 inflammasome activation in human lung epithelial cells. Part Fibre Toxicol. 2013;10:3.

89. Tran HB, Lewis MD, Tan LW, Lester SE, Baker LM, $\mathrm{Ng}$ J, et al. Immunolocalization of NLRP3 Inflammasome in Normal Murine Airway Epithelium and Changes following Induction of Ovalbumin-Induced Airway Inflammation. J Allergy (Cairo). 2012;2012:819176.

90. Hirota JA, Hirota SA, Warner SM, Stefanowicz D, Shaheen F, Beck PL, et al. The airway epithelium nucleotide-binding domain and leucine-rich repeat protein 3 inflammasome is activated by urban particulate matter. J Allergy Clin Immunol. 2012 Apr;129(4):1116-25 e6.

91. Cameron LA, Taha RA, Tsicopoulos A, Kurimoto M, Olivenstein R, Wallaert B, et al. Airway epithelium expresses interleukin-18. Eur Respir J. 1999 Sep;14(3):553-9. 
92. Pechkovsky DV, Goldmann T, Vollmer E, Muller-Quernheim J, Zissel G. Interleukin-18 expression by alveolar epithelial cells type II in tuberculosis and sarcoidosis. FEMS Immunol Med Microbiol. 2006 Feb;46(1):30-8.

93. Furugen M, Higa F, Hibiya K, Teruya H, Akamine M, Haranaga S, et al. Legionella pneumophila infection induces programmed cell death, caspase activation, and release of highmobility group box 1 protein in A549 alveolar epithelial cells: inhibition by methyl prednisolone. Respir Res. 2008;9:39.

94. Pittet JF, Koh H, Fang X, Iles K, Christiaans S, Anjun N, et al. HMGB1 accelerates alveolar epithelial repair via an IL-1beta- and alphavbeta6 integrin-dependent activation of TGF-beta1. PLoS One. 2013;8(5):e63907.

95. Lander AD, Selleck SB. The elusive functions of proteoglycans: in vivo veritas. J Cell Biol. 2000 Jan 24;148(2):227-32.

96. Shute JK, Solic N, Shimizu J, McConnell W, Redington AE, Howarth PH. Epithelial expression and release of FGF-2 from heparan sulphate binding sites in bronchial tissue in asthma. Thorax. 2004 Jul;59(7):557-62.

97. Perkins TN, Shukla A, Peeters PM, Steinbacher JL, Landry CC, Lathrop SA, et al. Differences in gene expression and cytokine production by crystalline vs. amorphous silica in human lung epithelial cells. Part Fibre Toxicol. 2012;9(1):6.

98. Hussain S, Sangtian S, Anderson SM, Snyder RJ, Marshburn JD, Rice AB, et al. Inflammasome activation in airway epithelial cells after multi-walled carbon nanotube exposure mediates a profibrotic response in lung fibroblasts. Part Fibre Toxicol. 2014 Jun 10;11(1):28.

99. Gillette DD, Shah PA, Cremer T, Gavrilin MA, Besecker BY, Sarkar A, et al. Analysis of human bronchial epithelial cell proinflammatory response to Burkholderia cenocepacia infection: inability to secrete il-1beta. J Biol Chem. 2013 Feb 8;288(6):3691-5.

100. Unno H, Futamura K, Morita H, Kojima R, Arae K, Nakae S, et al. Silica and Double-Stranded RNA Synergistically Induce Bronchial Epithelial Apoptosis and Airway Inflammation. Am J Respir Cell Mol Biol. 2014 Mar 24.

101. Allen IC, Jania CM, Wilson JE, Tekeppe EM, Hua X, Brickey WJ, et al. Analysis of NLRP3 in the development of allergic airway disease in mice. J Immunol. 2012 Mar 15;188(6):2884-93.

102. Triantafilou K, Kar S, van Kuppeveld FJ, Triantafilou M. Rhinovirus-induced calcium flux triggers NLRP3 and NLRC5 activation in bronchial cells. Am J Respir Cell Mol Biol. 2013 Dec;49(6):923-34.

103. Dostert C, Petrilli V, Van Bruggen R, Steele C, Mossman BT, Tschopp J. Innate immune activation through Nalp3 inflammasome sensing of asbestos and silica. Science. 2008 May 2;320(5876):674-7.

104. Willingham SB, Allen IC, Bergstralh DT, Brickey WJ, Huang MT, Taxman DJ, et al. NLRP3 (NALP3, Cryopyrin) facilitates in vivo caspase-1 activation, necrosis, and HMGB1 release via inflammasome-dependent and -independent pathways. J Immunol. 2009 Aug 1;183(3):2008-15.

105. Gasse P, Mary C, Guenon I, Noulin N, Charron S, Schnyder-Candrian S, et al. IL-1R1/MyD88 signaling and the inflammasome are essential in pulmonary inflammation and fibrosis in mice. $\mathrm{J}$ Clin Invest. 2007 Dec;117(12):3786-99. 
106. Gasse P, Riteau N, Charron S, Girre S, Fick L, Petrilli V, et al. Uric acid is a danger signal activating NALP3 inflammasome in lung injury inflammation and fibrosis. Am J Respir Crit Care Med. 2009 May 15;179(10):903-13.

107. Pauwels NS, Bracke KR, Dupont LL, Van Pottelberge GR, Provoost S, Vanden Berghe T, et al. Role of IL-1alpha and the Nlrp3/caspase-1/IL-1 beta axis in cigarette smoke-induced pulmonary inflammation and COPD. Eur Respir J. 2011 Nov;38(5):1019-28.

108. Eltom S, Stevenson CS, Rastrick J, Dale N, Raemdonck K, Wong S, et al. P2X7 receptor and caspase 1 activation are central to airway inflammation observed after exposure to tobacco smoke. PLoS One. 2011;6(9):e24097.

109. Fukumoto J, Fukumoto I, Parthasarathy PT, Cox R, Huynh B, Ramanathan GK, et al. NLRP3 deletion protects from hyperoxia-induced acute lung injury. Am J Physiol Cell Physiol. 2013 Jul 15;305(2):C182-9.

110. Ritter M, Straubinger K, Schmidt S, Busch DH, Hagner S, Garn H, et al. Functional relevance of NLRP3 inflammasome-mediated IL-1beta during acute allergic airway inflammation. Clin Exp Immunol. 2014 Jun 18.

111. Yamagata S, Tomita K, Sato R, Niwa A, Higashino H, Tohda Y. Interleukin-18-deficient mice exhibit diminished chronic inflammation and airway remodelling in ovalbumin-induced asthma model. Clin Exp Immunol. 2008 Dec;154(3):295-304.

112. Brusselle GG, Provoost S, Bracke KR, Kuchmiy A, Lamkanfi M. Inflammasomes in respiratory disease: from bench to bedside. Chest. 2014 May;145(5):1121-33.

113. Leemans JC, Kors L, Anders HJ, Florquin S. Pattern recognition receptors and the inflammasome in kidney disease. Nat Rev Nephrol. 2014 Jul;10(7):398-414.

114. Jenkins DA, Wojtacha DR, Swan P, Fleming S, Cumming AD. Intrarenal localization of interleukin-1 beta mRNA in crescentic glomerulonephritis. Nephrol Dial Transplant. 1994;9(9):1228-33.

115. Noronha IL, Kruger C, Andrassy K, Ritz E, Waldherr R. In situ production of TNF-alpha, IL-1 beta and IL-2R in ANCA-positive glomerulonephritis. Kidney Int. 1993 Mar;43(3):682-92.

116. Tesch GH, Yang N, Yu H, Lan HY, Foti R, Chadban SJ, et al. Intrinsic renal cells are the major source of interleukin-1 beta synthesis in normal and diseased rat kidney. Nephrol Dial Transplant. 1997 Jun;12(6):1109-15.

117. Bani-Hani AH, Leslie JA, Asanuma H, Dinarello CA, Campbell MT, Meldrum DR, et al. IL-18 neutralization ameliorates obstruction-induced epithelial-mesenchymal transition and renal fibrosis. Kidney Int. 2009 Sep;76(5):500-11.

118. Melnikov VY, Faubel S, Siegmund B, Lucia MS, Ljubanovic D, Edelstein CL. Neutrophilindependent mechanisms of caspase-1- and IL-18-mediated ischemic acute tubular necrosis in mice. J Clin Invest. 2002 Oct;110(8):1083-91.

119. Edelstein CL, Hoke TS, Somerset H, Fang W, Klein CL, Dinarello CA, et al. Proximal tubules from caspase-1-deficient mice are protected against hypoxia-induced membrane injury. Nephrol Dial Transplant. 2007 Apr;22(4):1052-61. 
120. Ponomareva L, Liu H, Duan X, Dickerson E, Shen H, Panchanathan R, et al. AIM2, an IFNinducible cytosolic DNA sensor, in the development of benign prostate hyperplasia and prostate cancer. Mol Cancer Res. 2013 Oct;11(10):1193-202.

121. Abdul-Sater AA, Koo E, Hacker G, Ojcius DM. Inflammasome-dependent caspase-1 activation in cervical epithelial cells stimulates growth of the intracellular pathogen Chlamydia trachomatis. J Biol Chem. 2009 Sep 25;284(39):26789-96.

122. Triantafilou K, Eryilmazlar D, Triantafilou M. Herpes simplex virus 2-induced activation in vaginal cells involves Toll-like receptors 2 and 9 and DNA sensors DAI and IFI16. Am J Obstet Gynecol. 2014 Feb;210(2):122 e1- e10.

123. Chang A, Ko K, Clark MR. The emerging role of the inflammasome in kidney diseases. Curr Opin Nephrol Hypertens. 2014 May;23(3):204-10.

124. Fang L, Xie D, Wu X, Cao H, Su W, Yang J. Involvement of endoplasmic reticulum stress in albuminuria induced inflammasome activation in renal proximal tubular cells. PLoS One. 2013;8(8):e72344.

125. Nishi Y, Satoh M, Nagasu H, Kadoya H, Ihoriya C, Kidokoro K, et al. Selective estrogen receptor modulation attenuates proteinuria-induced renal tubular damage by modulating mitochondrial oxidative status. Kidney Int. 2013 Apr;83(4):662-73. 



\title{
CHAPTER 6
}

\section{SILICA INDUCES NLRP3 INFLAMMASOME ACTIVATION IN HUMAN LUNG EPITHELIAL CELLS}

\author{
Paul M Peeters ${ }^{1,2}$, Timothy N Perkins ${ }^{1,2}$, Emiel F M Wouters ${ }^{1}$, Brooke T Mossman ${ }^{2}$, Niki L \\ Reynaert ${ }^{1}$ \\ ${ }^{1}$ Department of Respiratory Medicine, Maastricht University Medical Centre+ (MUMC+), Maastricht \\ University, Maastricht, The Netherlands \\ ${ }^{2}$ Department of Pathology, University of Vermont College of Medicine, 89 Beaumont Avenue, \\ Burlington, VT 05405, USA
}

Particle and Fibre Toxicology. 2013 Feb 12;10:3.

\begin{abstract}
Background: In myeloid cells the inflammasome plays a crucial role in innate immune defenses against pathogen- and danger-associated patterns such as crystalline silica. Respirable mineral particles impinge upon the lung epithelium causing irreversible damage, sustained inflammation and silicosis. In this study we investigated lung epithelial cells as a target for silica-induced inflammasome activation.

Methods: A human bronchial epithelial cell line (BEAS-2B) and primary normal human bronchial epithelial cells (NHBE) were exposed to toxic but nonlethal doses of crystalline silica over time to perform functional characterization of NLRP3, caspase-1, IL-1 $\beta$, bFGF and HMGB1. Quantitative RT-PCR, caspase-1 enzyme activity assay, western blot techniques, cytokine-specific ELISA and fibroblast (MRC-5 cells) proliferation assays were performed.

Results: We were able to show transcriptional and translational upregulation of the components of the NLRP3 intracellular platform, as well as activation of caspase-1. NLRP3 activation led to maturation of pro-IL-1 $\beta$ to secreted IL-1 $\beta$, and a significant increase in the unconventional release of the alarmins bFGF and HMGB1. Moreover, release of bFGF and HMGB1 was shown to be dependent on particle uptake. Small interfering RNA experiments using siNLRP3 revealed the pivotal role of the inflammasome in diminished release of proinflammatory cytokines, danger molecules and growth factors, and fibroblast proliferation.

Conclusion: Our novel data indicate the presence and functional activation of the NLRP3 inflammasome by crystalline silica in human lung epithelial cells, which prolongs an inflammatory signal and affects fibroblast proliferation, mediating a cadre of lung diseases.
\end{abstract}





\section{BACKGROUND}

Crystalline silica $\left(\mathrm{SiO}_{2}\right)$ is the second most common mineral in the earth's crust and is the major component of sand, rock and mineral ores. In occupational and environmental settings, microscopic mineral particles in airborne dust, generated by wind, manufacturing or demolition are inhaled and can get taken up by epithelial cells lining the respiratory tract, initiating and sustaining inflammatory responses at high concentrations. Prolonged exposure in the workplace may lead to the development of silicosis, which can be irreversible and is characterized by the development of progressive pulmonary fibrosis [1]. Crystalline silica exists in many different polymorphs, but those of particular concern are the naturally occurring polymorphs quartz, cristobalite and tridymite.

The Nacht Domain- Leucine-Rich Repeat-, and PYD-containing Protein 3 (NLRP3) inflammasome has recently been recognized as an innate immune signaling receptor important in mediating cell responses to various endo- and exogenous signals [2-4]. This multi- protein platform interacts with the apoptosis-associated speck-like protein PYCARD/ASC, which contains a caspase-1 recruitment domain. Detection of a danger signal such as crystalline silica particles in macrophages, activates the inflammasome complex leading to binding and activation of pro-caspase-1, which in turn causes the cleavage and secretion the of pro-inflammatory cytokines interleukin -1 beta (IL $-1 \beta)$ and interleukin -18 (IL -18$)$ to their active forms [5-7]. IL-1, a very potent and pivotal mediator of inflammatory responses induced by silica exposure $[8,9]$ affects virtually all tissue types and has been implicated in the pathophysiology of human and experimental silicosis [10,11]. Additionally, caspase-1 expression and activation is required for unconventional secretion of IL-33, IL-1 $\alpha$, basic fibroblast growth factor (bFGF) and high mobility group box 1 (HMGB1) although these proteins are not substrates of caspase-1 [12-15].

Until recently, it was assumed that the initial responses of lung tissue to silica were orchestrated mainly by cells of the innate immune system, such as monocytes, macrophages, neutrophils, and dendritic cells. Purified primary human monocyte-derived macrophages, peripheral blood mononuclear cells, and mouse bone marrow-derived macrophages release IL-1 $\beta$ after inflammasome activation by silica [6, 7]. NLRP3 knockout mouse models develop reduced pulmonary inflammation and less abundant collagen deposition compared to wildtype animals following silica administration [5]. Importantly, in these studies the localization of the active inflammasome in lung tissue was not shown.

Non-myeloid cells can also be important guardians for the detection of danger signals, fulfilling tasks usually performed by resident macrophages. Many groups have demonstrated NLRP3 presence and functional activity in non-myeloid cells by various agonists. For example, nanoparticles activate the NLRP3 inflammasome, leading to IL-1 $\beta$ secretion in primary human keratinocytes [16]. Recently, expression of the NLRP3 inflammasome in human airway epithelium following in vivo particulate matter exposure has been shown although its functional significance in lung disease was unknown [17]. Because the lung epithelial surface is one of the largest primary barriers to environmental exposures and the initial site of impingement of respirable silica, we hypothesized that bronchial epithelial cells were an important target of inflammasome activation. This activation may fuel cross-talk 
between neighboring fibroblasts, endothelial cells, as well as cells of the immune system which in turn release secondary mediators and initiate or mediate fibrogenesis.

\section{MATERIALS AND METHODS}

\section{BEAS-2B cell culture}

Non-tumorigenic human bronchial epithelial cells (Ad12-SV40 immortalized) BEAS 2B (ATCC, Manassas, VA) were grown and maintained in Dulbecco's Minimal Essential Medium (DMEM)/F12 containing 10\% Fetal Bovine Serum (FBS) (CellGro® Mediatech inc, Manassas, VA), with penicillin (50 U/ml), streptomycin $(100 \mu \mathrm{g} / \mathrm{ml})$ (Invitrogen, Carlsbad, CA), hydrocortisone $(100 \mu \mathrm{g} / \mathrm{ml})$, insulin $(2.5 \mu \mathrm{g} / \mathrm{ml})$, transferrin $(2.5 \mu \mathrm{g} / \mathrm{ml})$ and selenium $(2.5 \mu \mathrm{g} / \mathrm{ml})$ (Sigma, St. Louis, MO). Culture flasks and plates (BD, Franklin Lakes, NJ) were pre-coated with a mixture of fibronectin (Sigma, St. Louis, MO) $(0.01 \mathrm{mg} / \mathrm{ml})$, bovine collagen type I $(0.03 \mathrm{mg} / \mathrm{ml})$ (Invitrogen, Carlsbad, CA) and bovine serum albumin $(0.01$ $\mathrm{mg} / \mathrm{ml}$ ) (Sigma, St. Louis, MO), in DMEM/F12 media for 24 hours at $37^{\circ} \mathrm{C}$. Prior to exposures, medium was aspirated and replaced with reduction medium containing $0.5 \%$ FBS. In selected experiments BEAS-2B cells were primed with $5 \mu \mathrm{g} / \mathrm{mL}$ LPS for $4 \mathrm{~h}$ prior to silica exposure. Particle uptake was blocked by administration of $0,5 \mu \mathrm{g} / \mathrm{mL}$ cytochalasin $\mathrm{D}$ for $1 \mathrm{~h}$ prior to silica exposure.

\section{NHBE cell culture}

Primary normal human bronchial epithelial cells (NHBE-17917, Lonza, Clonetics ${ }^{\circledR}$ ) were cultured and maintained in BEGM ${ }^{\circledR}$ (Lonza, Clonetics ${ }^{\circledR}$. (Switzerland)) according to the manufacturers' protocol.

\section{MRC-5 cell culture}

The MRC-5 (CCL-171) cell line, a human fetal lung fibroblast cell line, was obtained from the ATCC (Rockville, MD) and maintained in Eagle's Minimum Essential Medium (Gibco) supplemented with L- Glutamine (200 mM, Invitrogen), $100 \mathrm{U} / \mathrm{ml}$ penicillin, $100 \mu \mathrm{g} / \mathrm{ml}$ streptomycin, and $10 \%$ heat-inactivated fetal calf serum (Gibco) and non-essential amino acids (MP Biomedicals) For addition of conditioned media, MRC-5 cells were serum starved for $24 \mathrm{~h}$ in Eagle's Minimum Essential Medium (Gibco) supplemented with L- Glutamine (200 $\mathrm{mM}$, Invitrogen), $100 \mathrm{U} / \mathrm{ml}$ penicillin, $100 \mu \mathrm{g} / \mathrm{ml}$ streptomycin, and $0,5 \%$ heatinactivated fetal calf serum (Gibco) and Non-essential amino acids (MP Biomedicals).

\section{THP-1 cell culture}

THP-1 cells obtained from ATCC (Rockville, MD) were grown in RPMI 1640 medium containing $10 \%$ fetal bovine serum with penicillin $(50 \mathrm{U} / \mathrm{ml})$, streptomycin $(100 \mu \mathrm{g} / \mathrm{ml})$ and 2 $\mathrm{mM}$ 1-glutamine at $37^{\circ} \mathrm{C}$. Ten ng/mL PMA was used to differentiate THP-1 cells for 24-36h prior to experiments.

\section{Particle exposures}

Cristobalite silica particles (C \& E Mineral Corp., King of Prussia, PA) were UV-irradiated over night to inactivate possible contaminating endotoxin. Silica particle suspensions $(1 \mathrm{mg} / \mathrm{mL})$ were sonicated for 15 minutes, aspirated 5 times through a 23 gauge needle and 
added to cell cultures. Throughout the studies presented in this paper, we utilized several particle doses based on their surface area characteristics and toxicity [18]. Glass beads (1-4 $\mu \mathrm{m}$ diameter), obtained from Particle Information Services, Inc. (Kingston,WA) were incorporated as a negative control based on particle surface area metrics.

\section{siRNA mediated knock down in BEAS-2B and THP-1 cells}

siRNA against NLRP3 (ON-TARGET plus SMARTpool L-017367-00-0005: GGAUCAAACUACUCUGUGA,UGCAAGAUCUCUCAGCAAA, AAGUGGGGUUCAGAUAAU, and GCAAGACCAAGACGUGUGA) and the ON-TARGET plus GAPD Control Pool (human); were purchased from Dharmacon, Thermo Scientific, USA. In BEAS-2B cells siRNA transfections were performed with Lipofectamine 2000 and $100 \mathrm{nM}$ siRNA $24 \mathrm{~h}$ prior to stimulations. THP-1 cells were differentiated with $10 \mathrm{ng} / \mathrm{mL}$ PMA over night after which 100 nM siRNA was transfected, using Lipofectamine 2000.

\section{RNA isolation}

Total RNA was prepared using an RNeasy® Plus Mini Kit according to the manufacturers' protocol (Qiagen, Valencia, CA) and $1 \mu \mathrm{g}$ was reverse-transcribed with random primers using the AMV Reverse Transcriptase kit (Pro-mega, Madison, WI). Primers for human NLRP3 were fw:GATCTTCGCTGCGATCAACAG and rev:CGTGCATTATCTGAACCCCAC. Primers used to determine caspase-1 mRNA levels were fw: TTTCCGCAAGGTTCGATTTTCA and rev: GGCATCTGCGCTCTACCATC. HPRT was used as the housekeeper gene (fw: GACCGGTTCTGTCATGTCG, rev: ACCTGGTTCATCATCACTAATCAC). Primer sequences were taken from Primerbank. Fold changes in the expression of genes of interest were calculated using the $\Delta \Delta \mathrm{Ct}$ method. Duplicate assays were performed with all samples.

\section{Western blots}

Cells were lysed in a buffer containing $20 \mathrm{mM}$ Tris, $150 \mathrm{mM} \mathrm{NaCl}, 1 \%$ Nonidet P-40, $1 \mathrm{mM}$ DTT, 1\% Protease Inhibitor Cocktail and 1\% Phosphatase Inhibitor Cocktail. Total protein content was determined by the Bio-Rad DC Protein Assay kit (Bio-Rad, Hercules, CA), and $20-40 \mu \mathrm{g}$ of protein was loaded onto polyacrylamide gels. After transfer of proteins to a nitrocellulose membrane, primary antibodies against caspase-1 (Santa Cruz Biotechnology, Santa Cruz, CA), $\beta$-actin (Cell Signaling Technologies), IL-1 $\beta$ (Cell Signaling Technologies), NLRP3 (Santa Cruz Biotechnology) or HMGB1 (Cell Signaling Technologies) were applied. After three washes with TBS-Tween, HRP conjugated secondary antibodies were detected by chemiluminescence according to the manufacturer's instructions. Densitometry was performed on scanned immunoblot images using the QuantityOne software (Bio-Rad Laboratories). Supernatants were concentrated using Amicon Ultra centrifugal filters (Millipore) and analyzed as described above.

\section{Caspase- 1 activity assay}

Caspase-1 activity was measured using a caspase-1 activity kit (R\&D) following the manufacturers' protocol. 


\section{ELISA}

The levels of IL-1 $\beta$ (Biolegend) and bFGF (Biolegend) in cell culture media were measured using human ELISA kits. HMGB1 was determined using a direct ELISA protocol. The primary antibody (ab18256 Abcam) was used at a 1/1000 dilution for 16 hours at $4^{\circ} \mathrm{C}$. The secondary antibody was a biotin-conjugated swine anti-rabbit used at a 1/1000 dilution. A blocking step was performed using 5\% BSA for 1 hour. Concentrations of IL- $1 \beta$, bFGF and HMGB1 were established via extrapolation from a standard curve of the appropriate recombinant protein.

\section{Proliferation assay}

BEAS-2B cells were treated with silica suspensions for $24 \mathrm{~h}$. Supernatants were collected and centrifuged to remove suspended particulates after which conditioned media was added to MRC- 5 cells at a 1 to 4 ratio. At indicated time points, MRC- 5 cells were fixed with ice cold $70 \%$ ethanol for $5 \mathrm{~min}$. After aspiration, $5 \mathrm{~N} \mathrm{NaOH}$ was added followed by DNA content measurement at $260 \mathrm{~nm}$.

\section{Statistical analyses}

All experiments were performed 3 times. Data were analyzed by one-way analysis of variance (ANOVA) using the Student Neuman-Keul's test to adjust for multiple pair-wise comparisons between treatment groups, or the Student's $t$ test where appropriate. Data from multiple experiments were averaged and expressed as mean values \pm SEM. Differences with $p$-values $<0.05$ were considered statistically significant.

\section{RESULTS}

Cristobalite silica particles induce increased mRNA levels and enzymatic activity of caspase-1 in primary human bronchial epithelial cells

Based on an available microarray dataset in the BEAS-2B cell line and primary human bronchial epithelial (NHBE) cells [18] we identified a group of genes related to the inflammasome as well as to the pro-inflammatory cytokine, IL-1 $\beta$ with significantly altered expression levels following $24 \mathrm{~h}$ exposure to cristobalite silica (Table 1). As described before, we worked with silica at $75 \times 10^{6} \mu \mathrm{m}^{2} / \mathrm{cm}^{2}$ on NHBE cells, a concentration with an approximate $25 \%$ cytotoxicity that allowed us to investigate the maximum inflammatory and possible fibrogenic effects of these mineral particles [18]. We next performed RT-PCR to investigate the quantitative mRNA expression levels of caspase-1 in NHBE cells. Figure 1A shows a significant increase of caspase-1 mRNA levels in primary human bronchial epithelial cells when exposed to $75 \times 10^{6} \mu \mathrm{m}^{2} / \mathrm{cm}^{2}$ (SIL75). NRLP3 mRNA levels were not enhanced in NHBE cells exposed to silica (data not shown). To investigate whether silica exposure in addition to increasing transcript levels of inflammasome-related genes would lead to the activation of pro-caspase-1, caspase-1 cleavage was determined by Western blotting. Figure 1B shows increases in protein levels of the functional p20 subunit of caspase-1 in NHBE whole cell lysates after treatment with SIL75 for $24 \mathrm{~h}$. Importantly, glass beads (GB, negative control), which are dense amorphous microspheres with decreased surface reactivity, did not 
induce increased levels of caspase- 1 cleaved subunits. A caspase- 1 activity assay confirmed its increased activation in NHBE cells exposed to SIL75 (Figure 1C).

Table 1: Selection of NLRP3 inflammasome and IL-1 family member related genes. For each cell type a selection of genes was collected from previously described microarray data set [18]. All transcripts are involved in inflammasome and IL-1 immune-cytokine responses.

\begin{tabular}{lll}
\hline BEAS-2B & & mRNA \\
Gene symbol & Gene name & Fold Change $\uparrow$ \\
\hline$I L 1 A$ & Interleukin 1, alpha & $6,2^{*}$ \\
$I L 1 R L 1$ & Interleukin 1 receptor-like 1 & 5,4 \\
$I L 1 B$ & Interleukin 1, beta & $3,6^{*}$ \\
$N L R P 1$ & NLR family, pyrin domain containing 1 & 3,4 \\
$N L R P 3$ & NLR family, pyrin domain containing 3 & $2,5^{*}$ \\
$I L 1 R L 2$ & Interleukin 1 receptor-like 2 & 2,3 \\
$I L 18 R 1$ & Interleukin 18 receptor 1 & 2,2 \\
$I L 1 R A P$ & Interleukin 1 receptor accessory protein & 2,2
\end{tabular}

\section{NHBE}

Gene symbol

IL1F9

TXNIP

ILIF5

IL32

ILIRL1

CASP1

$b F G F$

IL1A

\section{Gene name}

Interleukin 1 family, member 9

Thioredoxin interacting protein

Interleukin 1 family, member 5 (delta)

Interleukin 32

Interleukin 1 receptor-like 1

Caspase 1 (interleukin-1 beta convertase)

Fibroblast growth factor 2 (basic)

Interleukin 1, alpha
Fold Change $\uparrow$

14,0

9,7

6,0

5,5

3,5

$2,1 *$

2,1

2,1

* = confirmed by qRT-PCR 
A.

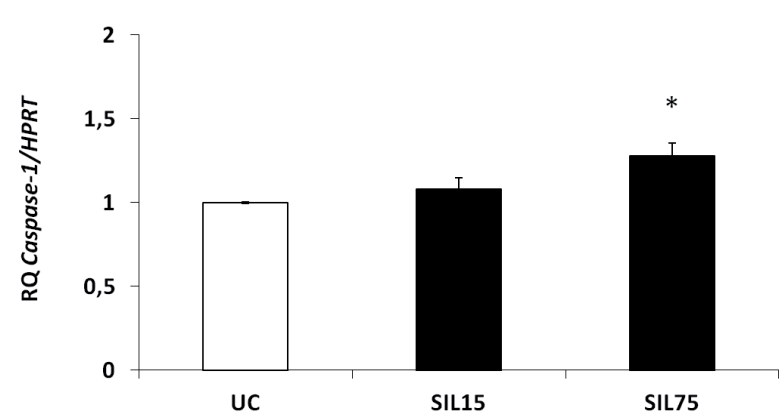

B.

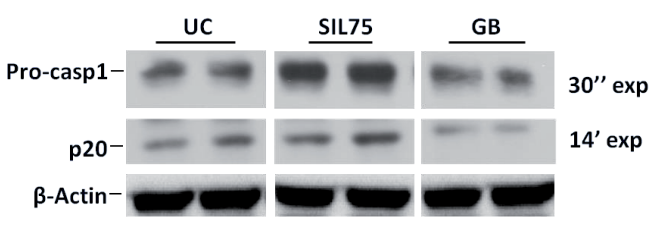

C.

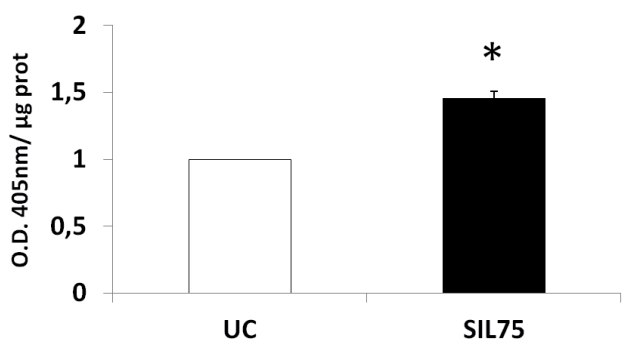

Figure 1: Cristobalite silica increases caspase-1 transcript levels and caspase -1 cleavage products in primary human bronchial epithelial cells. (A) Caspase-1 mRNA levels in primary normal human bronchial epithelial (NHBE) cells after $24 \mathrm{~h}$ exposure to 15 (SIL15) and $75 \mathrm{X} \mathrm{10}^{6} \mathrm{\mu m}^{2} / \mathrm{cm}^{2}$ (SIL75) cristobalite silica, expressed as mean fold change \pm SEM (indicated by error bars) with *p-value $<0.05$ compared to unexposed control cells (UC) and normalized to the housekeeping gene HPRT (N= 3). (B) Western blot analysis of caspase-1 cleaved products was conducted on whole cell lysates of NHBE cells after 24 h of exposure. p20 subunits were detected after a longer exposure compared to pro-caspase-1 (14 minutes versus 30 seconds). Glass beads (GB) and unexposed cells (UC) were included as controls, and $\beta$-actin was used as a loading control. (C) Caspase-1 enzymatic activity was assayed in cell lysates of NHBE cells exposed to the indicated silica concentrations for $24 \mathrm{~h}$ with. Caspase-1 activities are expressed as arbitrary units (O.D. $405 \mathrm{~nm} / \mu \mathrm{g}$ protein) with *p-value $<0.05$ compared to UC.

Cristobalite silica particles induce increased NLRP3 mRNA levels as well as elevated levels of cleaved caspase- 1 subunits

In BEAS-2B cells we first performed additional assays over a range of concentrations of cristobalite silica to determine toxicity (Supplemental figure 1). For further experiments, 
silica concentrations were used that were toxic, causing an acceptable maximum of $25 \%$ cell death. In contrast to NHBE cells, cristobalite silica was found to increase levels of NLRP3 mRNA dose dependently in BEAS-2B cells, after $24 \mathrm{~h}$ exposure (Figure 2A). In agreement with data in NHBE cells, we confirmed silica-induced caspase-1 activity in BEAS-2B cells following SIL150 exposure. Glass beads also did not affect caspase-1 activity significantly in these cells (Figure 2B).

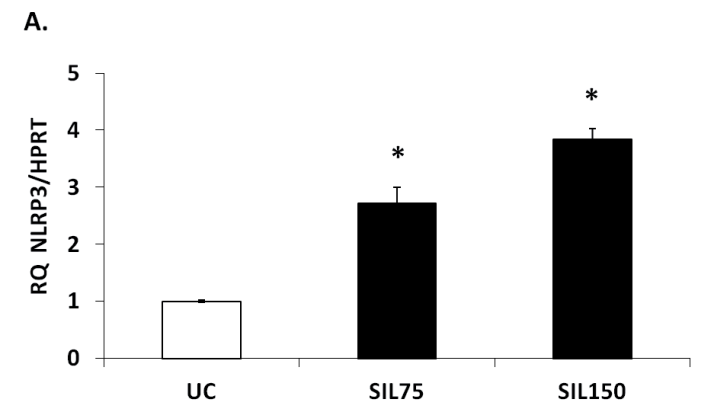

B.

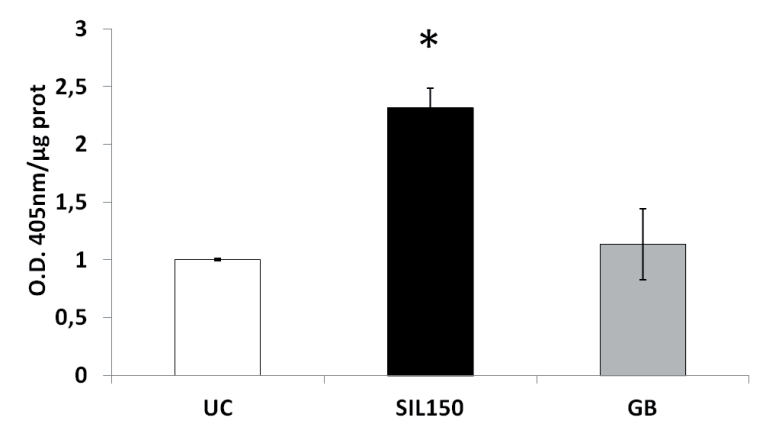

Figure 2: Crystalline silica increases NLRP3 mRNA levels and caspase-1 cleavage products in BEAS-2B cells. (A) Bar chart represents relative quantity of NLRP3 $m R N A$ levels after 24 h exposure to $75 \times 10^{6} \mathrm{\mu m}^{2} / \mathrm{cm}^{2}$ (SIL75) and $150 \times 10^{6} \mathrm{\mu m}^{2} / \mathrm{cm}^{2}$ (SIL150) of cristobalite silica in BEAS-2B cells. ${ }^{*} p$-value $<0.05$ compared to UC. (B) Caspase-1 enzymatic activity was assayed in cell lysates of $B E A S-2 B$ cells exposed to the indicated silica concentrations for $24 \mathrm{~h}$. Glass beads (GB) and unexposed cells (UC) were included as controls. Caspase-1 activities are expressed as arbitrary units (O.D. $405 \mathrm{~nm} / \mu \mathrm{g}$ total protein) with *p-value $<0.05$ compared to UC.

\section{Cytokine maturation and alarmin secretion in BEAS-2B cells in response to cristobalite silica}

Classically, inflammasome complex assembly, accompanied by caspase-1 activation leads to cleavage of pro-inflammatory IL- $1 \beta$ into its active form that is secreted. Interleukin (IL)-1 $\beta$ is produced by a variety of cell types in response to inflammatory stimuli such as lipopolysaccharide (LPS) [19]. In some studies it was shown that pro-IL1 $\beta$ mRNA levels first need to be elevated by a TLR-dependent priming stimulus, such as LPS before enhanced release of mature IL1 $\beta$ can be detected by an inflammasome stimulus. We therefore investigated whether silica-induced release of IL- $1 \beta$ requires LPS-priming in human bronchial 
epithelial cells, and whether LPS alone could trigger IL-1 $\beta$ maturation and release in BEAS2B cells. LPS administered to BEAS-2B cells showed only a moderate increase in detectable levels of the cleaved IL-1 $\beta$ subunits. Cells exposed to SIL150 demonstrated significantly increased intracellular maturation of pro-IL1 $\beta$. LPS priming, followed by silica exposure, did not further enhance silica-induced maturation of IL-1 $\beta$ (Figure 3A). We also investigated whether silica affected the release of alarmins linked to caspase-1 activation. A dosedependent increase in HMGB1 levels in culture medium supernatants $(\mathrm{SN})$ were detected following cristobalite exposure. Although LPS on its own induced a substantial increase of HMGB1 in media, LPS priming followed by silica exposure did not further augment HMGB1 levels in the medium compared to silica exposure alone (Figure 3B). We furthermore previously reported a significant dose-dependent release of bFGF from BEAS-2B cells following exposure to SIL150 for $24 \mathrm{~h}$ [18].

A.
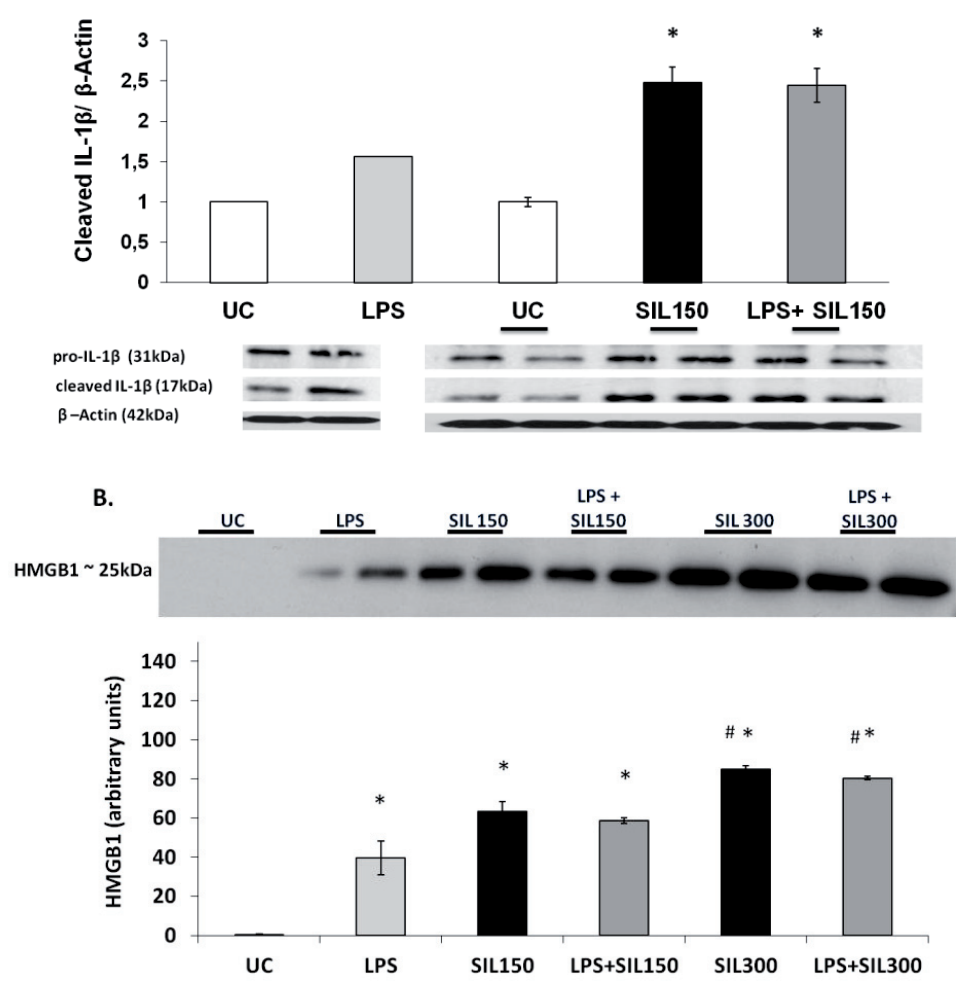

Figure 3: Silica augments processing and secretion of inflammatory mediators and DAMPs in bronchial epithelial cells. (A) Western blot analysis was conducted on whole cell lysates of BEAS- $2 B$ cells to detect cleaved IL-1 $\beta$ products, after $24 \mathrm{~h}$ of silica exposure with or without priming with $5 \mu \mathrm{g} / \mathrm{mL}$ LPS for $4 \mathrm{~h}$ ). Histogram represents results of densitometric analysis of cleaved IL-1 $\beta$ normalized to $\beta$-actin. (B) Western blot analysis of $H M G B 1$ release in the concentrated $S N$ from silica exposed BEAS-2B cells. Histogram represents results of semi-quantitative densitometric analysis of the HMGB1 signal expressed as arbitrary units. Data are presented as mean fold change \pm SEM with * p-value $<0.05$ compared to UC and \# p-value $<0.05$ compared to SIL150 or LPS +SIL150. 
The NLRP3 inflammasome mediates cristobalite silica-induced inflammatory danger signaling

In order to investigate whether cristobalite silica-induced release of IL1 $\beta$, HMGB1 and bFGF factors is mediated by the inflammasome, we performed RNAi-mediated knockdown of the NLRP3 gene. Transfection of NLRP3 siRNA in BEAS-2B cells was highly efficient in reducing NLRP3 mRNA levels $>50 \%$ as shown in Figure 4A. In parallel, the protein abundance of NLRP3 was reduced by transfection of NLRP3 siRNA (Figure 4B). As previously shown in Figure 3A, increased levels of IL-1 $\beta$ at SIL150 and an absence of a synergistic effect of LPS priming in the siRNA control group were found. Also, LPS priming alone did not induce significantly higher levels of IL-1 $\beta$ in the medium. The absence of effect of NLRP3 knock-down on basal $I L-1 \beta$ levels could be due to the activity of other enzymes including lysosomal enzymes and MMPs that can cleave $I L-1 \beta$ or the residual level of NLRP3. .Although priming with LPS still significantly enhanced IL-1 $\beta$ levels in response to silica in the NLRP3 siRNA transfected cells, these levels were significantly attenuated compared to the control siRNA transfected cells (Figure 4C). As alternative IL-1 $\beta$ processing enzymes mentioned above are known to be activated by silica and LPS, these alternative routes of processing could explain this attenuated, but not abrogated response in NRLP3 siRNA conditions. Increased levels of secreted bFGF by SIL150 could be further augmented by priming bronchial epithelial cells with LPS under control siRNA conditions, although not statistically significant. In all these conditions, bFGF levels were drastically attenuated after NLRP3 siRNA transfection, indicating a pivotal role for the inflammasome in bFGF release as seen in Figure 4D. The increased HMGB1 levels in medium caused by SIL150 alone, as well as after pre-treatment with LPS were also significantly reduced in the NLRP3 siRNA group (Figure 4E). 
A.

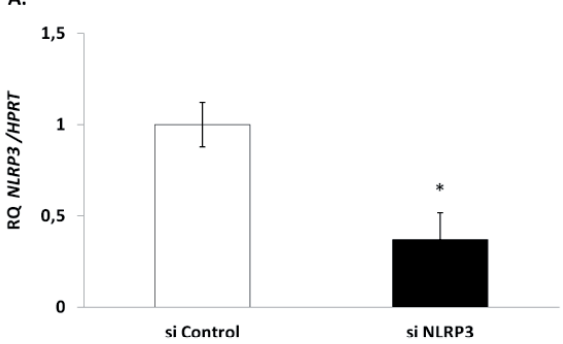

c.

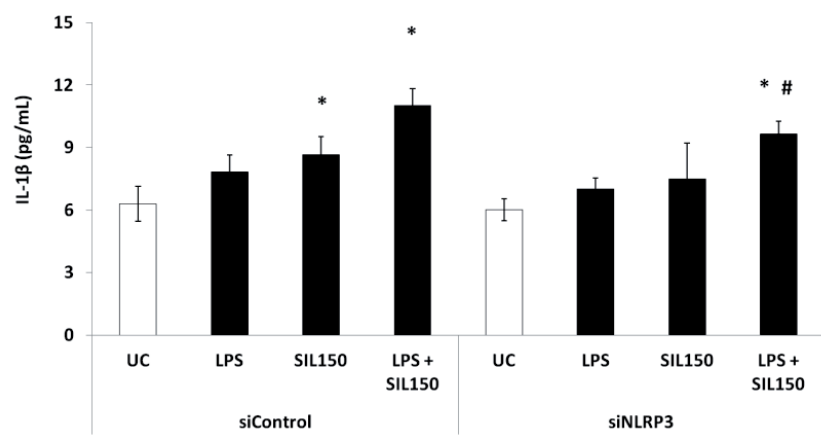

D.

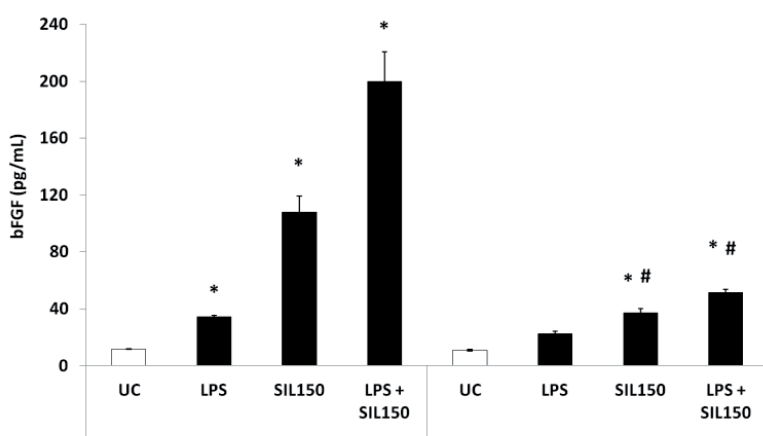

E.

si Control

Si NLRP3

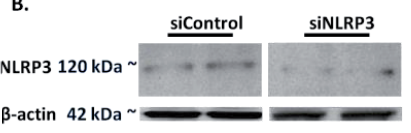

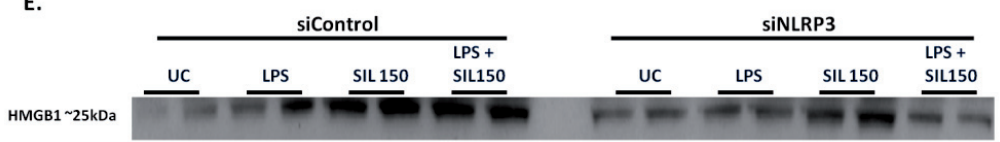

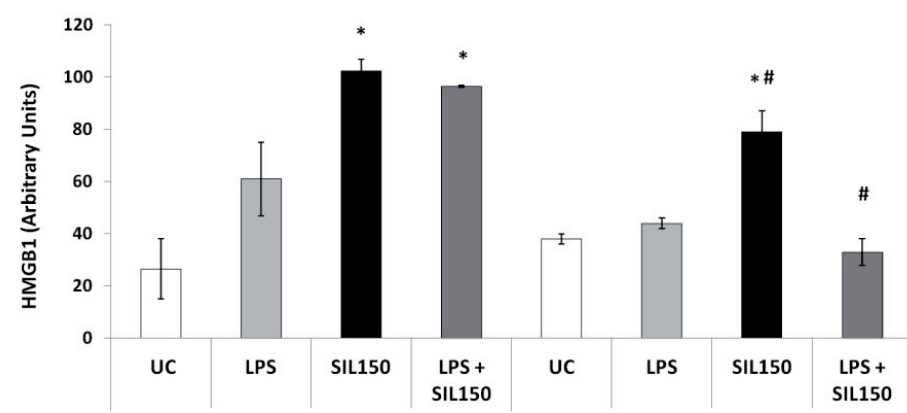


Figure 4: Canonical and non-canonical release of inflammatory mediators and DAMPs is regulated by NLRP3. BEAS- $2 B$ cells were transfected with control (siControl) or NLRP3 siRNA (siNLRP3). Experiments were performed 24 h after siRNA transfection. (A) Knockdown of NLRP3 $m R N A$ levels by siRNA. (B) Western blot analysis of whole cell lysates for the level of NLRP3 expression. Actin was used as a loading control. ELISA was performed on concentrated medium SN for detection of secreted $I L-1 \beta$ (C) and bFGF (D) 24 h post-transfection. The effect of LPS priming (5 $\mu \mathrm{g} / \mathrm{mL}$ ) was also investigated. (E) Western blot analysis, to detect influence of NLRP3 knockdown on HMGB1 levels, was conducted on concentrated medium supernatants. Histogram represents semiquantitative densitometric analysis of HMGB1 expressed as arbitrary units. Data are presented as mean fold change $\pm S E M$ with * p-value $<0.05$ compared to $U C$, and \# $p$-value $<0.05$ compared to the same treatment in the siControl setting.

\section{Comparison of inflammasome-dependent mediator release from bronchial epithelial cells versus macrophages in response to silica}

We conducted parallel experiments in BEAS-2B and THP-1 macrophages using the same dose of silica to investigate IL-1 $\beta$ levels and the effect of LPS priming on these cell types comparatively. Basal levels of secreted IL- $1 \beta$ are higher in THP-1 cells compared to BEAS2B $(28.3 \pm 2.7$ vs $6.29 \pm 0.5 \mathrm{pg} / \mathrm{ml}$ respectively). Silica induced IL-1 $\beta$ levels in BEAS-2B cells to $8.67 \pm 0.5 \mathrm{pg} / \mathrm{ml}$. In THP-1 cells silica alone also induced IL-1 $\beta$ release, to a level of $191.0 \pm 6.7 \mathrm{pg} / \mathrm{ml}$, about 22-fold higher compared to in BEAS-2B. LPS had no significant effect by itself on IL-1 $\beta$ levels in either cell type. In BEAS-2B there was no synergistic effect of priming with LPS prior to silica treatment, whereas this was the case in THP-1 cells. HMGB1 levels in culture medium were also approximately double in THP-1 cells compared to BEAS-2B cells, under baseline conditions as well as after silica stimulation. bFGF levels on the other hand were comparable in unstimulated cells, but induction was greater in bronchial epithelial cells (Supplemental table 1). The release of IL-1 $\beta$, HMGB1 and bFGF induced by silica was also NLRP3-dependent in THP-1 cells as indicated in Supplemental figure 2.

\section{Uptake dependence of inflammasome signaling}

To investigate if particle uptake was pivotal to silica-induced inflammasome activation and downstream readouts, actin polymerization was blocked using $0.5 \mu \mathrm{g} / \mathrm{mL}$ cytochalasin D for 1 h. Cytochalasin D administration for $1 \mathrm{~h}$ prior to silica exposure markedly attenuated increased bFGF and HMGB1 secretion (Figure 5A and 5B, respectively). Importantly, a loss of cell viability in the presence of cytochalasin D was not detected (data not shown). 

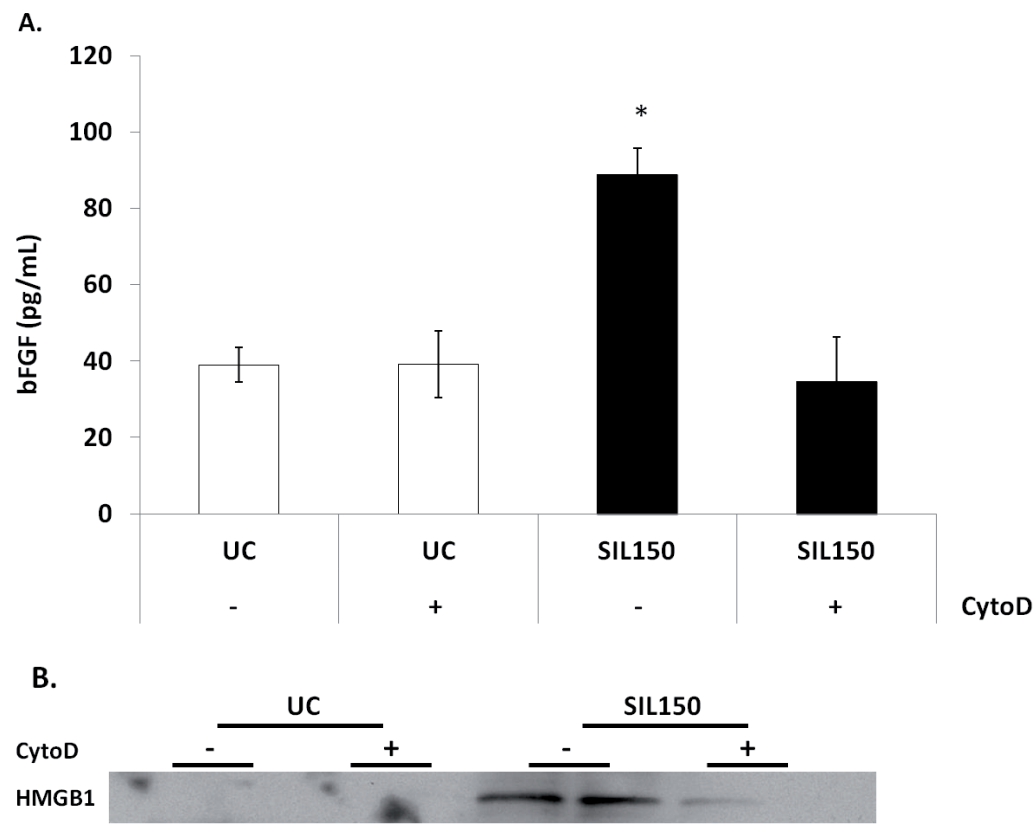

Figure 5: bFGF and HMGB1 release are particle uptake dependent. ELISA was performed on medium of BEAS-2B either or not pretreated with $0.5 \mu \mathrm{g} / \mathrm{mL}$ cytochalasin $D$ for $1 \mathrm{~h}$ measuring levels of $b F G F$ release (A). (B) Western blot analysis showing the effect of cytochalasin $D$ on the release of $H M G B 1$ in the medium following cristobalite silica exposure.

Inflammasome activity in bronchial cells is linked to proliferative effects on MRC-5 fibroblasts and is dependent on particle uptake

To elucidate whether the inflammasome mediated paracrine microenvironment signaling in our cell culture model, conditioned medium of BEAS-2B cells after exposure to silica was tested for fibroblast proliferative capacity. Indeed, the conditioned medium of silica-exposed epithelial cells drastically increased the DNA content of MRC-5 fibroblasts up to 4 days after administration compared to conditioned medium of untreated controls. This proliferative effect was dependent on silica uptake as media from epithelial cells with cytochalasin D administration prior to silica addition failed to induce fibroblast proliferation (Figure 6A). Likewise, conditioned medium from NLRP3 siRNA transfected epithelial cells blunted higher DNA content levels in MRC-5 fibroblasts to baseline values (Figure 6B), indicating a role for the inflammasome in the initiation of neighboring fibroblast proliferation in response to silica. Conditioned media of LPS treated BEAS-2B cells did not affect fibroblast proliferation (Supplemental figure 3). 
A.

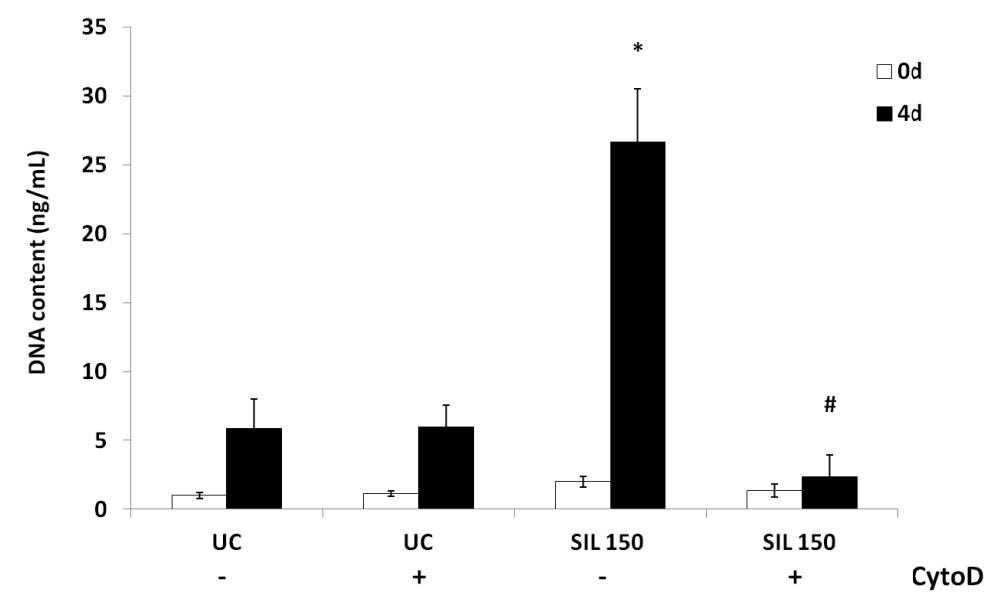

B.

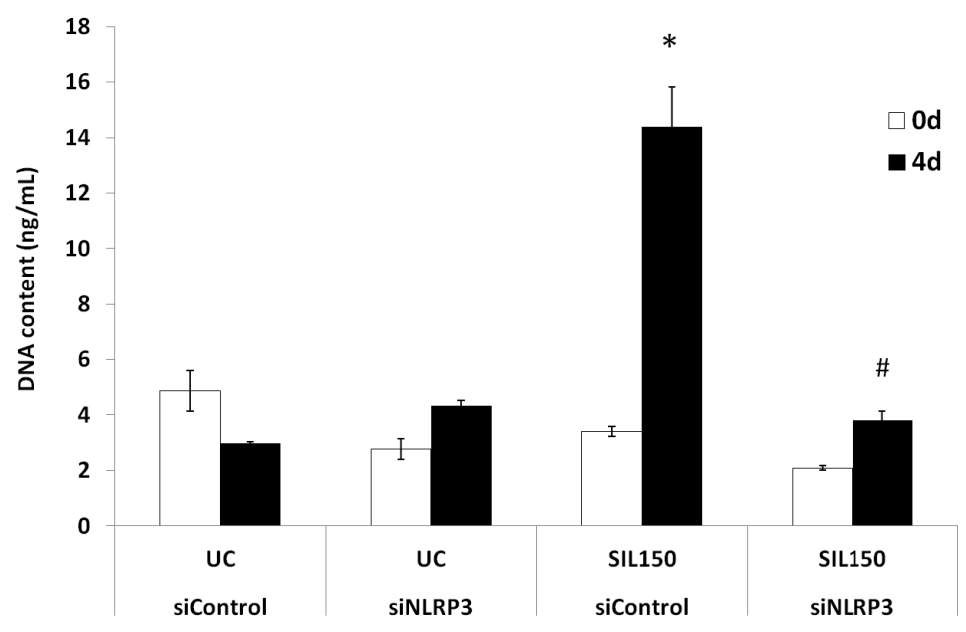

Figure 6: Fibroblast proliferation is affected by exposure to bronchial epithelial cell conditioned media. Conditioned BEAS-2B media was added to a monolayer of MRC-5 fibroblast cells and DNA content was measured up to 4 days to give an indication of proliferation. (A) MRC-5 proliferation is impacted by inhibition of particulate uptake in BEAS-2B medium. (B) Fibroblast proliferation after exposure to conditioned media of siRNA transfected BEAS-2B cells. *indicate a statistically significant difference in DNA content compared to UC at each time point, whereas \# $p<0.05$ indicates significant difference compared to the positive control. $N=3$ for each time point in each media condition. 


\section{DISCUSSION}

In myeloid cells, the inflammasome receptor complex is recognized as one of the cornerstones of the long-sought intracellular surveillance system [20]. The NLRP3 (NALP3) complex contributes to innate immune defenses against pathogen- and danger-associated moieties such as exogenous or endogenous crystalline molecules [5-7, 21].

Our present study assessed the importance of the bronchial epithelial cells as a target for cristobalite silica-induced inflammasome activation. Thereby, classical and non-canonical outputs of NLRP3 assembly and caspase-1 activation might contribute to the development of silicotic nodules and fibrosis. This study illuminated increases in transcription of components of the NLRP3 multiprotein platform which leads to caspase-1 activation in response to cristobalite silica in bronchial epithelial cells.

It has been postulated that the release of active IL-1 needs at first a signal provided by Tolllike receptor (TLR) engagement that results in gene transcription and pro-IL-1 $\beta$ accumulation, a process sometimes described as "priming"; and a second signal through NLR signaling, which results in caspase-1 activation and subsequently processing of this cytokine into mature bioactive IL-1 $\beta$. Nevertheless, it is still controversial whether there is a direct role for TLR signaling in the activation of the inflammasome because of contrasting results regarding the requirement of a "two-hit-model" in the assembly of the multiprotein complex. Epithelial cell monolayers, like some myeloid cells, do not always require LPS priming in contrast with the well documented 'two hit model' and therefore may provoke a constant phase of alarm when bombarded with cristobalite particles. It is therefore not surprising to find only modest but significantly increased levels of the most potent pyrogen IL-1 $\beta$, in whole cell lysates or medium of cell cultures after treatment with silica without priming with LPS. Although in the comparative experiments using THP-1 macrophages silica induced a 22 -fold higher IL-1 $\beta$ release compared to the response in BEAS-2B cells, many more epithelial cells are present in the lungs compared to macrophages. Together with the observations that ambient particulate matter $\mathrm{PM}_{10}$ alone could induce increases of detectable cleaved subunits of IL-1 $\beta$ in bronchial epithelial cells [17] and described IL-1 $\beta$ release from bronchial epithelial cells after cigarette smoke and rhinovirus without priming with LPS [22, 23], bronchial epithelial cells can be considered danger sensing cells contributing to small but relevant levels of biologically active IL-1 $\beta$.

The nuclear-damage-associated molecular pattern molecule (DAMP), HMGB1 was originally identified as one of the nuclear proteins mediating gene transcription as a chromatin binder [24]. In secreted form, it is thought to be a cytokine-like molecule derived from various cell types, and acting as an alarmin. Furthermore, it induces production of pro-inflammatory cytokines [25]. Activated inflammasomes are involved in the release of HMGB1 through unconventional protein secretion, or as a soluble molecule from cells during pyroptosis [15]. HMGB1 is involved in innate and specific immune responses and contributes to acute lung injury in bleomycin-induced fibrosis in mice [26]. The same study also demonstrated that HMGB1 stimulates proliferation of human fibroblasts without effects on apoptosis and directly participated in fibrogenesis. Additionally, HMGB1 seems to protect the host from pathogen-induced mortality [27] and has the ability to attract stem cells to areas inflammation, thus promoting their regeneration $[28,29]$. Here we show that inflammasome activation 
mediates unconventional HMGB1 release from BEAS-2B and THP-1 cells following early interactions with silica and as a consequence of further amplification of the reaction cascade. bFGF is released after tissue injury and during inflammation and is produced by lung epithelial cells, macrophages and endothelial cells [30]. It plays a crucial role during fibrosis as well as angiogenesis [31, 32]. We recently showed higher transcript levels and bFGF protein secretion in cristobalite silica treated BEAS-2B cells compared to untreated controls [18]. Here, we extended these findings by demonstrating inflammasome dependence of release of bFGF from epithelial cells and macrophages suggesting an early role for the inflammasome in initiating fibrogenesis.

In the previous study, we demonstrated uptake of cristobalite silica in bronchial epithelial cells [18]. Therefore, we investigated whether inflammasome activation was dependent on uptake of particles as shown by others in myeloid cells [5-7, 21]. Decreased levels of bFGF and HMGB1 release occurred in medium after cytochalasin D pre-treatment, indicating a pivotal role for internalization of silica in inflammasome activation and release of proinflammatory and fibrotic mediators. It remains to be elucidated how particulates are internalized and how this triggers inflammasome assembly and activation.

Finally, possible effects of inflammasome activation in epithelial cells on the lung microenvironment were examined. It was found that lung epithelial cells after internalization of silica secrete substances, including bFGF that induce fibroblast proliferation in vitro. The fact that the inhibition of particle uptake and inflammasome inactivation in bronchial epithelial cells dramatically blunted fibroblast proliferative response is a novel observation of direct relevance to fibrogenesis.

The role of IL-1 $\beta$ in fibrosis is widely studied because IL-1 $\beta$ can induce its own gene expression and chronic activation via IL-1 receptor signaling. This could result in the continual cleavage of IL-1 $\beta$ via a positive feedback mechanism that could conceivably maintain a profibrotic phenotype. Uptake-related and inflammasome-dependent HMGB1 and bFGF secretion from lung epithelial cells suggest that crystalline silica induces local cellular injury which intensifies both disturbed immune signaling and increased proliferative capacity of cells during fibrogenesis (Figure 7). 

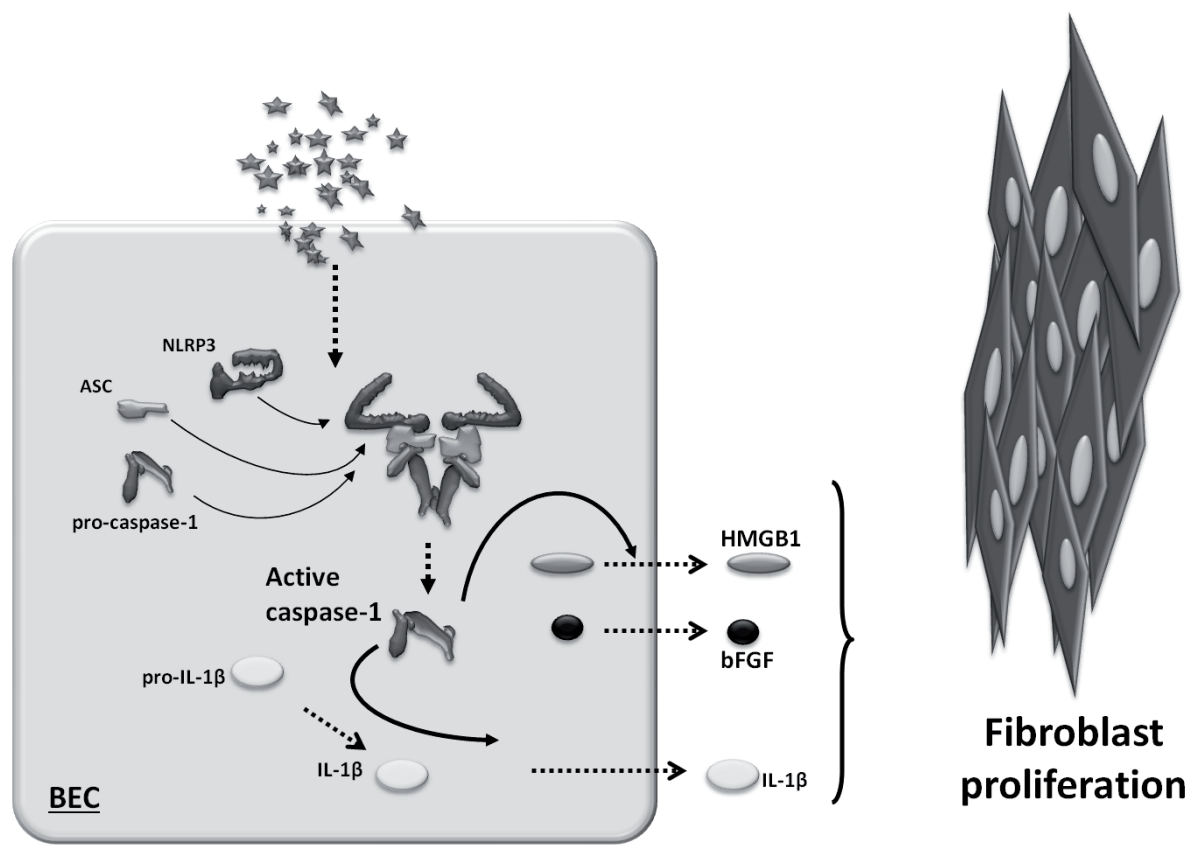

Figure 7: A schematic representation of our in vitro model. In bronchial epithelial cells (BEC), cristobalite silica particles initiate the production of inflammatory cytokines, alarmins and growth factors leading to fibroblast proliferation in a particle uptake and NLRP3 inflammasome-dependent manner.

Data presented here are the result of in vitro experiments and it remains to be investigated whether bronchial epithelial cells in vivo are a major site of silica-induced inflammasome activation and consequent signaling. Recently, it was published that human bronchial epithelial cells express functional NLRP3 [17] following $\mathrm{PM}_{10}$ exposure in a mouse model. Also, they demonstrated NLRP3-dependent airway neutrophilia and increases in dendritic cell numbers in intrathoracic lymph nodes, indicating the influence of NLRP3 on different aspects of immune cell signaling.

Collectively, our data indicate that bronchial epithelial cells are much more active than previously assumed. The NLRP3 inflammasome is activated in a one-step fashion, leading to downstream occurring events that are important in relaying the danger signal to the microenvironment contributing to the pathological condition of silicosis.

\section{CONCLUSION}

These novel data show the presence and functional activation of the NLRP3 inflammasome by cristobalite silica in human lung epithelial cells that mediates a cadre of lung diseases. Moreover, these findings show that inflammasome activation by silica in epithelial cells is linked to fibroblast proliferation. 


\section{REFERENCES}

1. BT Mossman, A Churg: Mechanisms in the pathogenesis of asbestosis and silicosis. Am J Respir Crit Care Med 1998, 157:1666-80.

2. F Martinon, K Burns, J Tschopp: The inflammasome: a molecular platform triggering activation of inflammatory caspases and processing of proIL-beta. Mol Cell 2002, 10:417-26.

3. K Schroder, J Tschopp: The inflammasomes. Cell 2010, 140:821-32.

4. BK Davis, H Wen, JP Ting: The inflammasome NLRs in immunity, inflammation, and associated diseases. Annual review of immunology 2011, 29:707-35.

5. SL Cassel, SC Eisenbarth, SS Iyer, JJ Sadler, OR Colegio, LA Tephly, AB Carter, PB Rothman, RA Flavell, FS Sutterwala: The Nalp3 inflammasome is essential for the development of silicosis. Proc Natl Acad Sci U S A 2008, 105:9035-40.

6. C Dostert, V Petrilli, R Van Bruggen, C Steele, BT Mossman, J Tschopp: Innate immune activation through Nalp3 inflammasome sensing of asbestos and silica. Science 2008, 320:6747.

7. V Hornung, F Bauernfeind, A Halle, EO Samstad, H Kono, KL Rock, KA Fitzgerald, E Latz: Silica crystals and aluminum salts activate the NALP3 inflammasome through phagosomal destabilization. Nat Immunol 2008, 9:847-56.

8. GS Davis, LM Pfeiffer, DR Hemenway: Persistent overexpression of interleukin-1beta and tumor necrosis factor-alpha in murine silicosis. Journal of environmental pathology, toxicology and oncology : official organ of the International Society for Environmental Toxicology and Cancer 1998, 17:99-114.

9. KE Driscoll, JK Maurer: Cytokine and growth factor release by alveolar macrophages: potential biomarkers of pulmonary toxicity. Toxicologic pathology 1991, 19:398-405.

10. B Rimal, AK Greenberg, WN Rom: Basic pathogenetic mechanisms in silicosis: current understanding. Current opinion in pulmonary medicine 2005, 11:169-73.

11. F Huaux: New developments in the understanding of immunology in silicosis. Current opinion in allergy and clinical immunology 2007, 7:168-73.

12. CA Dinarello: An IL-1 family member requires caspase-1 processing and signals through the ST2 receptor. Immunity 2005, 23:461-2.

13. J Schmitz, A Owyang, E Oldham, Y Song, E Murphy, TK McClanahan, G Zurawski, M Moshrefi, J Qin, X Li, et al: IL-33, an interleukin-1-like cytokine that signals via the IL-1 receptor-related protein ST2 and induces T helper type 2-associated cytokines. Immunity 2005, 23:479-90.

14. M Keller, A Ruegg, S Werner, HD Beer: Active caspase-1 is a regulator of unconventional protein secretion. Cell 2008, 132:818-31.

15. L Vande Walle, TD Kanneganti, M Lamkanfi: HMGB1 release by inflammasomes. Virulence 2011, 2:162-5.

16. AS Yazdi, G Guarda, N Riteau, SK Drexler, A Tardivel, I Couillin, J Tschopp: Nanoparticles activate the NLR pyrin domain containing 3 (Nlrp3) inflammasome and cause pulmonary 
inflammation through release of IL-1alpha and IL-1beta. Proc Natl Acad Sci U S A 2010, 107:19449-54.

17. JA Hirota, SA Hirota, SM Warner, D Stefanowicz, F Shaheen, PL Beck, JA Macdonald, TL Hackett, DD Sin, S Van Eeden, et al: The airway epithelium nucleotide-binding domain and leucine-rich repeat protein 3 inflammasome is activated by urban particulate matter. The Journal of allergy and clinical immunology 2012, 129:1116-25 e6.

18. TN Perkins, A Shukla, PM Peeters, JL Steinbacher, CC Landry, SA Lathrop, C Steele, NL Reynaert, EF Wouters, BT Mossman: Differences in gene expression and cytokine production by crystalline vs. amorphous silica in human lung epithelial cells. Particle and fibre toxicology 2012, 9:6.

19. CA Dinarello: Interleukin-1 and interleukin-1 antagonism. Blood 1991, 77:1627-52.

20. VA Rathinam, SK Vanaja, KA Fitzgerald: Regulation of inflammasome signaling. Nature immunology 2012, 13:333-2.

21. F Martinon, V Petrilli, A Mayor, A Tardivel, J Tschopp: Gout-associated uric acid crystals activate the NALP3 inflammasome. Nature 2006, 440:237-41.

22. C Rusznak, PR Mills, JL Devalia, RJ Sapsford, RJ Davies, S Lozewicz: Effect of cigarette smoke on the permeability and IL-1beta and sICAM-1 release from cultured human bronchial epithelial cells of never-smokers, smokers, and patients with chronic obstructive pulmonary disease. Am J Respir Cell Mol Biol 2000, 23:530-6.

23. L Shi, DM Manthei, AG Guadarrama, LY Lenertz, LC Denlinger: Rhinovirus-induced IL-1beta release from bronchial epithelial cells is independent of functional P2X7. Am J Respir Cell Mol Biol 2012, 47:363-71.

24. S Muller, L Ronfani, ME Bianchi: Regulated expression and subcellular localization of HMGB1, a chromatin protein with a cytokine function. Journal of internal medicine 2004, 255:332-43.

25. HE Harris, U Andersson, DS Pisetsky: HMGB1: a multifunctional alarmin driving autoimmune and inflammatory disease. Nature reviews. Rheumatology 2012, 8:195-202.

26. N Hamada, T Maeyama, T Kawaguchi, M Yoshimi, J Fukumoto, M Yamada, S Yamada, K Kuwano, Y Nakanishi: The role of high mobility group box1 in pulmonary fibrosis. American journal of respiratory cell and molecular biology 2008, 39:440-7.

27. SB Willingham, IC Allen, DT Bergstralh, WJ Brickey, MT Huang, DJ Taxman, JA Duncan, JP Ting: NLRP3 (NALP3, Cryopyrin) facilitates in vivo caspase-1 activation, necrosis, and HMGB1 release via inflammasome-dependent and -independent pathways. Journal of immunology 2009, 183:2008-15.

28. H Naglova, M Bucova: HMGB1 and its physiological and pathological roles. Bratislavske lekarske listy 2012, 113:163-71.

29. W Yan, Y Chang, X Liang, JS Cardinal, H Huang, SH Thorne, SP Monga, DA Geller, MT Lotze, A Tsung: High-mobility group box 1 activates caspase-1 and promotes hepatocellular carcinoma invasiveness and metastases. Hepatology 2012, 55:1863-75. 
30. JI Herseth, V Volden, PE Schwarze, M Lag, M Refsnes: IL-1beta differently involved in IL-8 and FGF-2 release in crystalline silica-treated lung cell co-cultures. Particle and fibre toxicology 2008, 5:16.

31. Y Inoue, TE King, Jr., SS Tinkle, K Dockstader, LS Newman: Human mast cell basic fibroblast growth factor in pulmonary fibrotic disorders. The American journal of pathology 1996, 149:2037-54.

32. M Przybylski: A review of the current research on the role of bFGF and VEGF in angiogenesis. Journal of wound care 2009, 18:516-9.

\section{SUPPLEMENTAL MATERIAL}

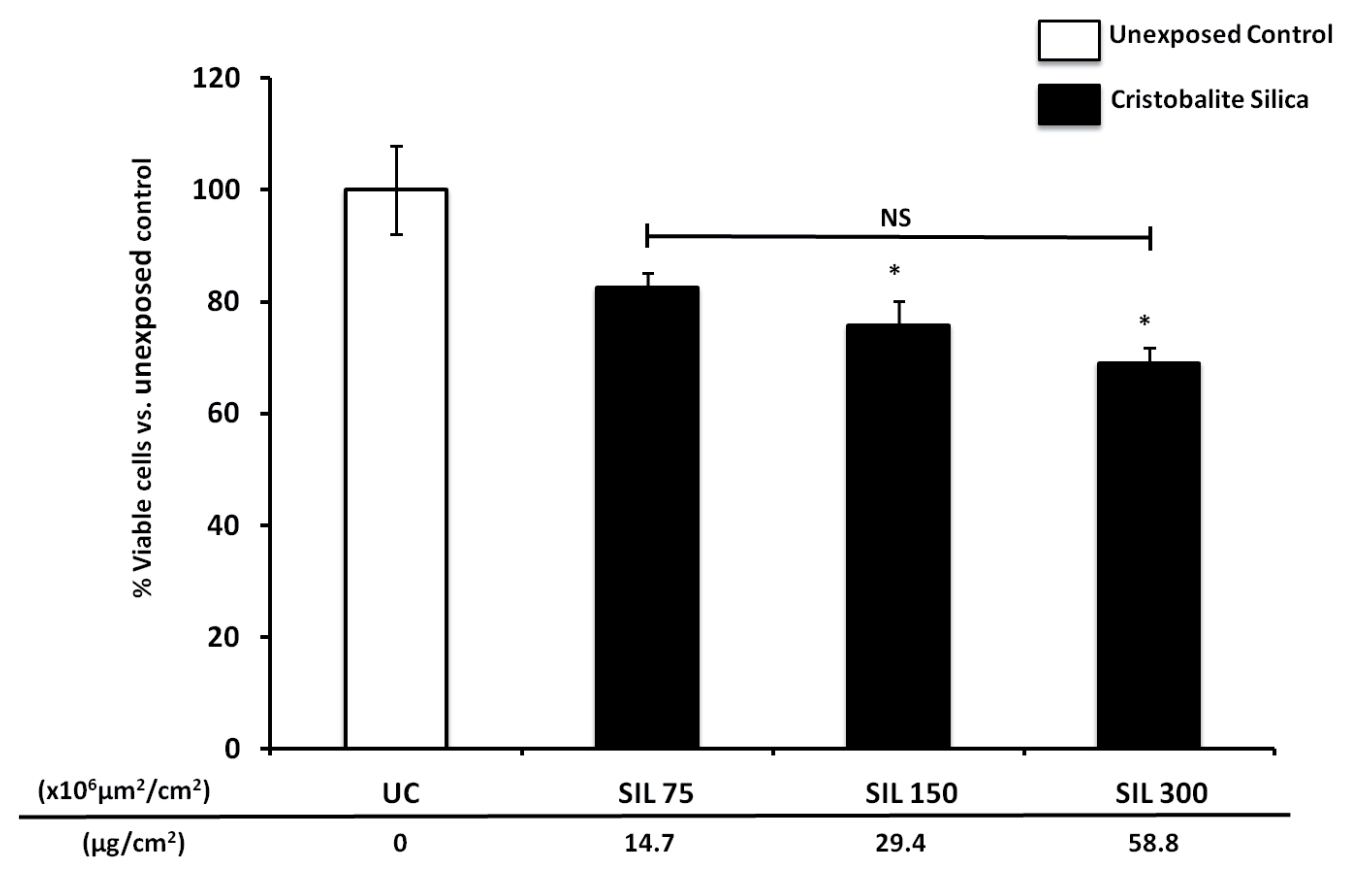

Supplemental figure 1: Assessment of BEAS-2B cell viability after exposure to silica particles for 24h. Cell viability was assessed by the trypan blue exclusion assay. Results are expressed as the mean percent viable cells \pm SEM compared to unexposed controls and are representative of 3 independent experiments ( $N=3$ in each experiment). Surface area concentrations and mass concentrations of particles are expressed as $x 10^{6} \mathrm{\mu m}^{2} / \mathrm{cm}^{2}$ and $\mu \mathrm{g} / \mathrm{cm}^{2}$ respectively. * represents $p<0.05$ compared to $U C$. 

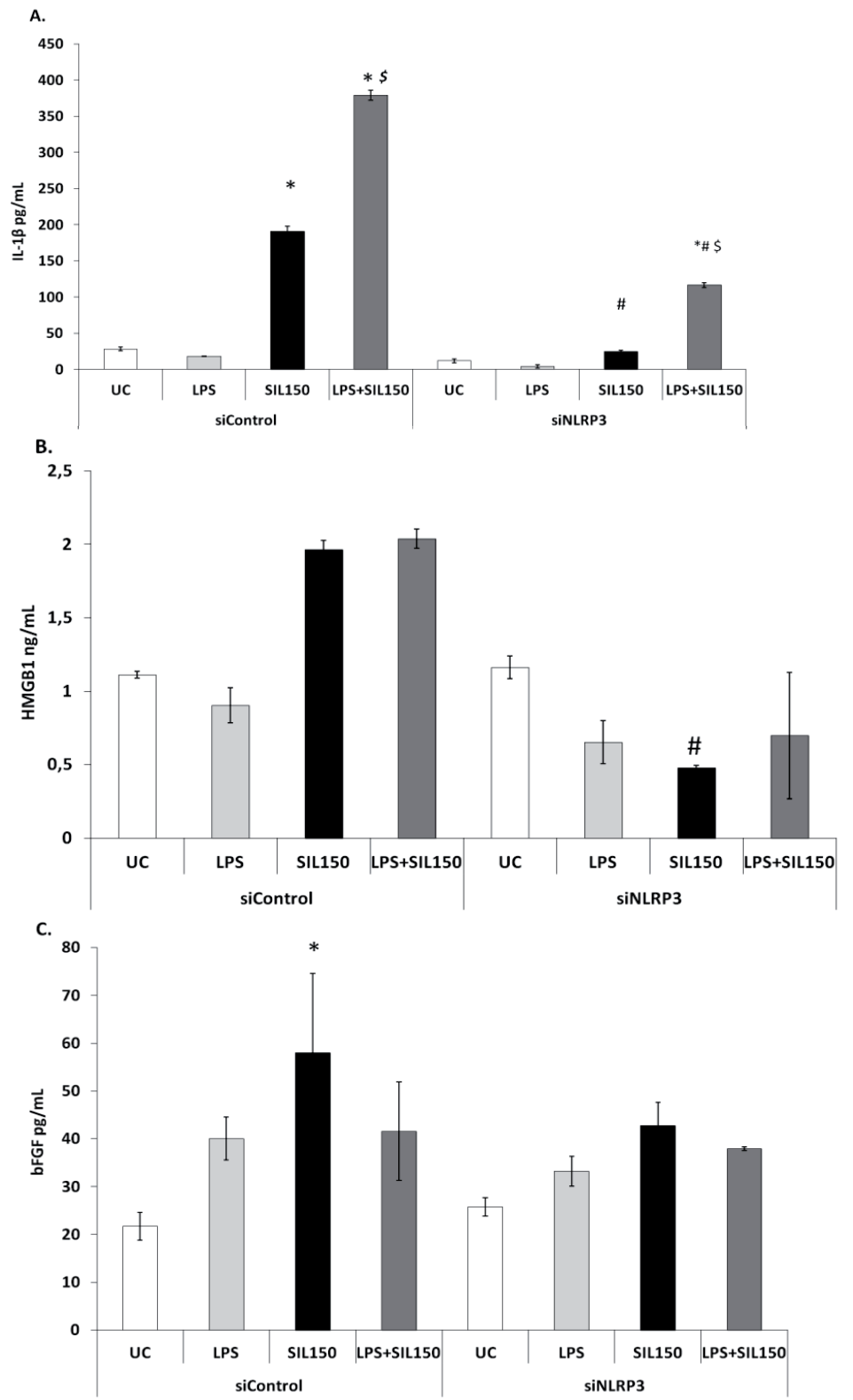

Supplemental figure 2: Silica-induced release of inflammatory mediators and DAMPs from THP-1 differentiated macrophages is NLRP3 dependent. ELISA performed on concentrated medium SN of $P M A$ differentiated macrophages for detection of secreted IL-1 $\beta$ (A), HMGBI (B) and bFGF (C) $24 \mathrm{~h}$ after silica treatment with or without priming with $5 \mu \mathrm{g} / \mathrm{mL}$ LPS for $4 \mathrm{hr}$. Data are presented as mean $\pm S E M$ with * p-value $<0.05$ compared to UC, \# p-value $<0.05$ compared to the siControl group and \$ p-value $<0,05$ compared to SIL150 alone. 


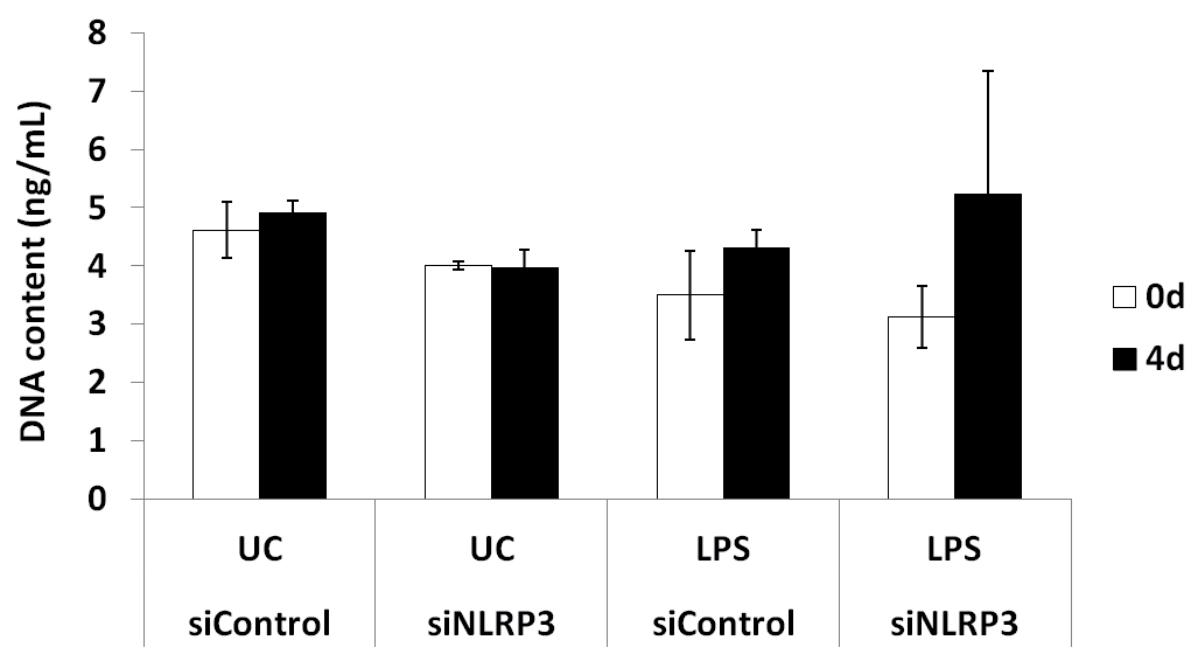

Supplemental figure 3: Fibroblast proliferation is not affected by exposure to LPS-treated BEAS$2 B$ conditioned medium.

Supplemental table 1: Secreted levels of IL-1ß, HMGB1 and bFGF from BEAS-2B and THP-1 cells in response to silica treatment measured by ELISA. Data represented show IL-1 $\beta, H M G B 1$ and bFGF levels (pg/mL) under baseline conditions and after exposure to $150 \times 10^{6} \mu \mathrm{m}^{2} / \mathrm{cm}^{2}$ cristobalite silica (SIL150) for 24h in BEAS-2B and THP-1 cells. * represents p-value <0,05 for the difference between UC and SIL150 in each cell type. \# represents p-value $<0,05$ for the difference in concentration between both cell types.

\begin{tabular}{|c|c|c|c|c|}
\hline & \multicolumn{2}{|c|}{ BEAS-2B } & \multicolumn{2}{|c|}{ THP-1 } \\
\hline & UC & SIL 150 & UC & SIL 150 \\
\hline IL-1 $\beta$ & $6,29 \pm 0,49$ & $8,67 \pm 0,49 *$ & $28,30 \pm 2,72^{\#}$ & $190,97 \pm 6,72 * \#$ \\
\hline HMGB1 & $283,58 \pm 70,84$ & $694,42 \pm 130,65$ & $1110 \pm 22,82^{\#}$ & $1962,36 \pm 63,75$ *\# \\
\hline bFGF & $11,65 \pm 0,11$ & $107,95 \pm 11,44^{*}$ & $21,67 \pm 2,93$ & $57,98 \pm 16,59$ \\
\hline
\end{tabular}





\title{
CHAPTER 7
}

\section{SILICA-INDUCED NLRP3 INFLAMMASOME ACTIVATION IN VITRO AND IN RAT LUNGS}

\author{
P.M.Peeters ${ }^{12}$, I.M.J. Eurlings ${ }^{1}$, T.N. Perkins ${ }^{1}$, E.F.Wouters ${ }^{1}$, R.P.F Schins ${ }^{2}$, P.J.A. Borm ${ }^{2}$, W. \\ Drommer $^{4}$, N.L.Reynaert ${ }^{1}$, C. Albrecht ${ }^{2}$ \\ 1. Department of Respiratory Medicine, Maastricht University Medical Centre+ (MUMC+), \\ Maastricht University, Maastricht, The Netherlands; \\ ${ }^{2}$. IUF - Leibniz Research Institute for Environmental Medicine, Düsseldorf, Düsseldorf, Germany \\ ${ }^{3}$. Bèta Sciences and Technology, Hogeschool Zuyd, Heerlen, The Netherlands \\ ${ }^{4}$. Histo-Path GmbH, Hannover, Germany
}

\section{Particle and Fibre Toxicology (In Press)}

\begin{abstract}
Rationale: Mineral particles in the lung cause inflammation and silicosis. In myeloid and bronchial epithelial cells the inflammasome plays a role in responses to crystalline silica. Thioredoxin (TRX) and its inhibitory protein TRX-interacting protein link oxidative stress with inflammasome activation. We investigated inflammasome activation by crystalline silica polymorphs and modulation by TRX in vitro, as well as its localization and the importance of silica surface reactivity in rats. Methods: We exposed bronchial epithelial cells and differentiated macrophages to silica polymorphs quartz and cristobalite and measured caspase-1 activity as well as the release of IL-1beta, bFGF and HMGB1; including after TRX overexpression or treatment with recombinant TRX. Rats were intratracheally instilled with vehicle control, Dörentruper quartz (DQ12) or DQ12 coated with polyvinylpyridine Noxide. At days 3, 7, 28, 90, 180 and 360 five animals per treatment group were sacrificed. Hallmarks of silicosis were assessed with Haematoxylin-eosin and Sirius Red stainings. Caspase-1 activity in the bronchoalveolar lavage and caspase-1 and IL-1 $\beta$ localization in lung tissue were determined using Western blot and immunohistochemistry (IHC). Results: Silica polymorphs triggered secretion of IL$1 \beta, \mathrm{bFGF}$ and HMGB1 in a surface reactivity dependent manner. Inflammasome readouts linked with caspase-1 enzymatic activity were attenuated by TRX overexpression or treatment. At day 3 and 7 increased caspase-1 activity was detected in BALF of the DQ12 group and increased levels of caspase1 and IL-1 $\beta$ were observed with IHC in the DQ12 group compared to controls. DQ12 exposure revealed silicotic nodules at 180 and 360 days. Particle surface modification markedly attenuated the grade of inflammation and lymphocyte influx and attenuated the level of inflammasome activation, indicating that the development of silicosis and inflammasome activation is determined by crystalline silica surface reactivity. Conclusion: Our novel data indicate the pivotal role of surface reactivity of crystalline silica to activate the inflammasome in cultures of both epithelial cells and macrophages. Inhibitory capacity of the antioxidant TRX to inflammasome activation was evidenced. DQ12 quartz exposure induced acute and chronic functional activation of the inflammasome in the heterogeneous cell populations of the lung in associated with its crystalline surface reactivity.
\end{abstract}





\section{INTRODUCTION}

Exposure to respirable crystalline silica dusts is very common in a multitude of industries and occupational settings. Inhalation of fine silica, including their most abundant naturally occurring polymorphs quartz and cristobalite, can lead to progressive pulmonary fibrosis and silicosis which continues to be a problem in developed and in third world countries [1]. The presence and severity of the disease depends on many factors such as individual susceptibility, and the level and duration of exposure [2]. Pathophysiologically, many molecular and micro-environmental reactions lead to sustained inflammation, prolonged tissue damage and remodeling, which over time often lead to the development of progressive interstitial fibrosis and silica-induced lung cancer [3,4].

In recent years the role of the inflammasome pathway in silica-induced lung inflammation and tissue remodeling has been emerging [5-7]. The nucleotide-binding oligomerization domain receptor, in short NOD-like receptor (NLR), pyrin domain-containing 3 (NLRP3) inflammasome belongs to the NLR subfamily of pattern-recognition receptors [8]. This multiprotein complex consists of the NLRP3 protein itself, the adapter molecule Apoptosisassociated Speck-like protein containing a CARD (ASC) and caspase-1. Assembly of these proteins leads to conversion of the pro-inflammatory cytokines IL- $1 \beta$ and IL-18 into their active forms by caspase- 1 and release of growth factors and alarmins including basic fibroblast growth factor (bFGF) and High mobility group protein B1 (HMGB1) into the extracellular space [9-11]. In murine models of silicosis ASC-, NLRP3- and IL-1 $\beta$-deficient mice are more resistant to the infiltration of inflammatory cells, the formation of granulomas, excess collagen deposition and the development of fibrosis in response to silica instillation [6]. Moreover, it has been demonstrated that treatment with an IL-1 receptor antagonist is effective in reducing established silica-induced pulmonary fibrosis and that neutralization of IL-1 $\beta$ could attenuate silica-induced lung inflammation and fibrosis in mice $[12,13]$. These data indicate that the activation of the NLRP3 inflammasome by silica is key in the development of silicosis in mice. These studies did not however examine inflammasome activation in the time course of silicosis development, nor the cell types in which it occurred. In vitro, crystalline silica-induced inflammasome activation has been investigated profoundly in effector cells of the immune system; for example macrophages treated with silica have been shown to secrete IL-1 $\beta$ and IL-18 in an NLRP3-dependent manner [5-7]. Furthermore, we previously demonstrated induction of inflammasome signaling in lung epithelial cells in vitro in response to exposure to cristobalite silica in association with inflammatory and profibrogenic mediator release in the epithelial microenvironment [14]. The exact mechanism(s) by which silica particles trigger the assembly of the inflammasome complex still remains to be elucidated. One of the proposed mechanisms links lysosomal rupture and increased intracellular reactive oxygen species (ROS) levels to the assembly of the NLRP3 multi protein complex [7,15]. Other studies have shown an association between mitochondrial ROS production and regulation of the TXNIP/thioredoxin (TRX) axis to inflammasome activation $[5,16,17]$.

The particle surface reactivity of crystalline silica has been long recognized as an important feature to their pathogenic hazard as reviewed by several authors $[18,19]$. Effects of silica quartz on human lung epithelial cells and rat lung epithelial cells and macrophages have been 
shown to be determined by its surface reactivity. Exemplary, surface modification using various compounds including aluminium or the polymer polyvinylpyridine N-oxide (PVNO) drastically affected their ability to generate ROS and the downstream activation of proinflammatory signaling, as well as the development of pulmonary inflammation, toxicity and fibrosis in vitro and in vivo [20-25].

The aim of this study was to comparatively investigate inflammasome activation by cristobalite silica and native quartz at equal surface area metrics in different target cells in vitro and to determine if surface reactivity is pivotal to this activation. In conjunction, it was investigated whether inflammasome activation could be prevented by enhancing cellular TRX levels. Secondly, although the importance of inflammasome activation to the initiation of inflammation and development of fibrosis has been shown in mice, it has not been elucidated in which cellular compartments activation occurs, whether it persists, and if surface reactivity of the particles is necessary. Therefore inflammasome activation was investigated in lungs of rats at multiple time points after exposure to quartz and the influence of the particle surface properties to this response was assessed by comparative evaluation of effects after PVNO polymer coated quartz instillation. 


\section{METHODS}

\section{In vitro experiments}

$B E A S-2 B$ cell culture

The non-tumorigenic Ad12-SV40 immortalized human bronchial epithelial cell line BEAS 2B (ATCC, Manassas, VA) was grown and maintained in Dulbecco's Minimal Essential Medium (DMEM)/F12 containing 10\% Fetal Bovine Serum (FBS) (CellGro ${ }^{\circledR}$ Mediatech inc, Manassas, VA), with penicillin (50 U/ml), streptomycin $(100 \mu \mathrm{g} / \mathrm{ml})$ (Invitrogen, Carlsbad, CA), hydrocortisone $(100 \mu \mathrm{g} / \mathrm{ml})$, insulin $(2.5 \mu \mathrm{g} / \mathrm{ml})$, transferrin $(2.5 \mu \mathrm{g} / \mathrm{ml})$ and selenium $(2.5 \mu \mathrm{g} / \mathrm{ml})$ (Sigma, St. Louis, MO). Culture flasks and plates (BD, Franklin Lakes, NJ) were pre-coated with a mixture of fibronectin (Sigma, St. Louis, MO) $(0.01 \mathrm{mg} / \mathrm{ml})$, bovine collagen type I $(0.03 \mathrm{mg} / \mathrm{ml})$ (Invitrogen, Carlsbad, CA) and bovine serum albumin (BSA, $0.01 \mathrm{mg} / \mathrm{ml}$, Sigma, St. Louis, MO) in DMEM/F12 media for $24 \mathrm{~h}$ at $37^{\circ} \mathrm{C}$. Prior to exposures, medium was replaced with medium containing $0.5 \%$ FBS for 4 hours.

\section{THP-1 cell culture}

The macrophage like cell line THP-1(ATCC) was grown in RPMI 1640 medium containing $10 \%$ FBS with penicillin $(50 \mathrm{U} / \mathrm{ml})$, streptomycin $(100 \mu \mathrm{g} / \mathrm{ml})$ and $2 \mathrm{mM} \mathrm{L}$-glutamine at $37^{\circ} \mathrm{C}$. Ten $\mathrm{ng} / \mathrm{mL}$ phorbol myristate acetate (PMA) was used to differentiate THP-1 cells for $72 \mathrm{~h}$ prior to experiments.

\section{Surface Modification of Quartz Particles and exposures}

To modify the surface properties, DQ12 quartz (Batch 6, IUF, Düsseldorf) particles were treated with PVNO (DQ12-PVNO) as described previous [24]. Briefly, quartz was suspended at a concentration of $5 \mathrm{mg} / \mathrm{mL}$ in $1 \%$ solutions of PVNO in distilled water, subsequently sonicated for $5 \mathrm{~min}$, and agitated for $3 \mathrm{~h}$ at room temperature. Quartz suspended in distilled water, sonicated and agitated for the same time intervals, was used as a control. The samples were then dried and stored in aliquots in the dark until use. The surface modification procedure did not affect the size distributions of the quartz sample as confirmed by electron microscopy analysis (shown in Albrecht et al. AJRCMB 2004). The in vitro experiments were performed with Cristobalite silica (C \& E Mineral Corp., King of Prussia, PA), the shamcoated DQ12 quartz and the PVNO coated DQ12 (DQ12-PVNO). All particles for in vitro experiments were UV-irradiated over night to inactivate possible contaminating endotoxin. Immediately before treatment of the cell cultures, silica particle suspensions $(1 \mathrm{mg} / \mathrm{mL})$ were sonicated for $15 \mathrm{~min}$, aspirated 5 times through a 23 gauge needle and added to cell cultures. Concentrations are shown in the figures as $\mu \mathrm{m}^{2}$ particle surface area per $\mathrm{cm}^{2}$ cell surface area on the basis of the BET surface area.

\section{Assessment of Cell Viability}

After $24 \mathrm{~h}$ of exposure, cells were collected by trypsinisation (Invitrogen, Carlsbad, CA) and non-viable cells were stained using trypan blue (MP Biomedicals, Solon, OH). The proportions of stained versus unstained viable cells were determined using a hemocytometer as described previously [26]. 
TRX overexpression and recombinant protein treatment in BEAS-2B and THP-1 cells

In BEAS-2B cells, transfections were performed using Extreme gene (Roche) and $1 \mu \mathrm{g}$ DNA $24 \mathrm{~h}$ prior to stimulations. Differentiated THP-1 cells were transfected with $1 \mu \mathrm{g}$ DNA using Lipofectamine 2000 (Invitrogen). Flag-tagged human thioredoxin overexpression plasmid was a kind gift of Dr. Haendeler, Heinrich-Heine University, Dusseldorf, Germany [27]. Recombinant human TRX protein was bought from Abfrontier (Korea).

\section{Caspase-1 activity assay}

Caspase-1 activity was measured using a commercially available assay (R\&D) following the manufacturer's protocol.

\section{ELISA}

Media samples were concentrated via aceton precipition with 2 volumes of ice cold acetone per volume of medium followed by 30 minutes at $-20^{\circ} \mathrm{C}$ and centrifugation for 10 minutes at $13.000 \mathrm{G}$. The obtained pellet was resuspended in $200 \mu$ d dilution buffer. The levels of IL-1 $\beta$ (Biolegend) and bFGF (Biolegend) in this concentrated cell culture media were measured using commercially available ELISAs. HMGB1 was determined using a direct ELISA protocol. In brief, after a blocking step using 5\% BSA for 1hour, the primary HMGB1 antibody (ab18256 Abcam) was incubated at a 1/1000 dilution for 16 hours at $4^{\circ} \mathrm{C}$. The secondary antibody was a biotin-conjugated swine anti-rabbit used at a 1/1000 dilution. Concentrations of IL-1 $\beta$, bFGF and HMGB1 were established via extrapolation from a standard curve of the appropriate recombinant protein.

\section{BALF concentration}

$500 \mu \mathrm{L}$ of BALF was concentrated by adding $500 \mu \mathrm{L}$ of methanol and $125 \mu \mathrm{L}$ of Chloroform followed by vortexing and 10 minutes centrifugation at 20,000xg. Next, the upper phase was removed and another $500 \mu \mathrm{L}$ of methanol was added to the sample followed by vortexing and 5 min centrifugation at $20,000 \mathrm{xg}$. The supernatants were discarded and the pellet was dried for $15 \mathrm{~min}$ at $55^{\circ} \mathrm{C}$ after which it was resuspended in $4 \mathrm{X}$ sample buffer and boiled for $5 \mathrm{~min}$ at $95^{\circ} \mathrm{C}$ for subsequent Western blotting.

\section{Western blot}

BEAS-2B cells were lysed in a buffer containing $20 \mathrm{mM}$ Tris, $150 \mathrm{mM} \mathrm{NaCl}, 1 \%$ [vol/vol] Nonidet P-40, $1 \mathrm{mM}$ DTT, $1 \%$ [vol/vol] Protease Inhibitor Cocktail, $1 \%$ [vol/vol] Phosphatase Inhibitor Cocktail. Total protein content was determined by the Bio-Rad DC Protein Assay kit (Bio-Rad, Hercules, CA), according to manufacturer's instructions. $20 \mu \mathrm{g}$ protein for WCL or $15 \mu \mathrm{L}$ of concentrated lavage or cell culture supernatants was loaded onto polyacrylamide gels. After transfer of proteins to a nitrocellulose membrane, primary antibodies against thioredoxin (ab16965, Abcam -kindly gifted by Dr. Haendeler) and caspase-1 (sc-56036, Santa Cruz) were applied at dilution of 1:1500 and 1:300 respectively, followed by HRP conjugated secondary antibodies. SuperSignal west femto maximum sensitivity ECL Substrate was used to visualize the proteins of interest (Thermo Scientific) and images were taken on the AIDA image analyzer. 


\section{In vivo experiments}

\section{Animals}

Female Wistar rats (8 weeks old, Janvier, Le Genest St Isle, France) were used for this study. The animals were housed and maintained in an accredited on-site testing facility, according to the guidelines of the Society for Laboratory Animals Science (GV-SOLAS). All animals were allowed food and water ad libitum. The animals were housed on hardwood bedding in plastic cages in an air-conditioned animal room $\left(23 \pm 2^{\circ} \mathrm{C}\right)$ with a regular $12 \mathrm{~h}$ light/dark cycle. The ethical approval for the animal study application was given by the North Rhine-Westphalia State Agency for Nature, Environment and Consumer Protection (NRW - LANUV, 23.05230-3-27/00). The intratracheal instillation procedures were performed after anesthetization of the animals using Isofluran (Essex Pharma GmbH, Munich, Germany). For to the instillation, aliquots of DQ12 or DQ12-PVNO were resuspended in PBS at a concentration of $5 \mathrm{mg} / \mathrm{ml}$. The suspensions were sonicated $(5 \mathrm{~min})$ and stirred until instillation by applying a total dose of $2 \mathrm{mg}$ in $0.4 \mathrm{ml}$ PBS. Control animals received $0.4 \mathrm{ml}$ PBS which was sonicated and stirred in the absence of particles.

\section{Animal treatment and histopathology}

Rats were sacrificed in deep anesthetisation by pentobarbital at day 3, 7, 28, 90, 180 and 360 after one single intratracheal instillation of PBS, DQ12 (2 mg) or DQ12-PVNO (2 mg) as previously published [21]. The lungs of five animals per treatment group were lavaged as described in detail elsewhere from independent rats that were not included for histopathology analysis [21,28]. The lavage fluid was spun at $500 \mathrm{xg}$ for $10 \mathrm{~min}\left(4^{\circ} \mathrm{C}\right)$ and cells were collected for enumeration of total and differential cell counts; the total recovery of the BALF was $90-95 \%$ of the instilled PBS volum. Supernatants were spun again $\left(900 \mathrm{xg}, 10 \mathrm{~min}, 4^{\circ} \mathrm{C}\right)$ and investigated for proteinand and inflammatory parameters. Thioredoxin and caspase-1 cleavage products have been measured in concentrated BALF. Lung fixation was performed in 5 independent animals per treatment group, for all treatment time intervals, by airway in situ perfusion of 4\% Paraformaldehyde/PBS pH 7.4 at $20 \mathrm{~cm} \mathrm{H}_{2} \mathrm{O}$ pressure. Lungs were embedded in paraffin and $5 \mu \mathrm{m}$ sections were cut for histopathological evaluation as well as immunohistochemistry.

Two sections of two lobes per animal - the left as well as the right cranial - were stained with hematoxylin \& eosin (H\&E) as well as Sirius red to investigate histopathological alterations. On H\&E stained slides the following alterations were evaluated according to severity and distribution, based on the approach used by Porter et al (28) as detailed in Additional file1 in the online supplement: broncho-alveolar hyperplasia, perivascular and peribronchiolar lymphocytic infiltration, alveolar histocytosis; lympho-histiocytosis, infiltration near hyperplasia, alveolar lipoproteinosis, cholesterol granulomas and mixed cell alveolar inflammation. Sirus red staining indicates collagen deposition and was used to score perivascular fibrosis, fibrosis in alveolar septa and granulomas.

\section{Immunohistochemistry}

Antigen retrieval was performed by using citrate buffer $\left(\mathrm{pH}\right.$ 6) for $10-15$ minutes at $100^{\circ} \mathrm{C}$ on deparaffinized and re 
hydrated sections. Blocking with 5\% BSA/TBS for $1 \mathrm{~h}$ at room temperature to prevent nonspecific binding was next performed. Following optimization, caspase-1 (Santa Cruz, SC56036), IL-1 $\beta$ (SC-7884) and HMGB1 primary rabbit anti-rat antibodies (Abcam, ab18256) were diluted in $0.5 \% \mathrm{BSA} / 0.1 \%$ tween/TBS to final concentrations of $2.5 \mu \mathrm{g} / \mathrm{ml}, 5.0 \mu \mathrm{g} / \mathrm{ml}$ and $0.5 \mu \mathrm{g} / \mathrm{ml}$ respectively. After 1 hour incubations with these antibodies, sections were washed $4 \mathrm{x}$ with $0.1 \% \mathrm{Tw} / \mathrm{TBS}$. The secondary antibody (swine anti-rabbit - biotin) was diluted $400 \mathrm{x}$ in $0.1 \% \mathrm{BSA} / \mathrm{TBS}$ and incubated for 30 minutes at room temperature. The washing step was repeated and $\mathrm{ABC} / \mathrm{AP}$ (200x diluted in $0.1 \% \mathrm{Tw} / \mathrm{TBS}$ ) was used for 30 minutes at room temperature. After washing, Vector Blue (50x diluted in $0.1 \mathrm{M}$ Tris $(\mathrm{pH}=8.2)$ ) was added to visualize the antibody complexes. The slides are washed for 5 minutes with tap water after which nuclei were stained with Nuclear Fast Red. Finally tissue sections were treated with histosafe (3x3 minutes) and mounted with vectamount. Images are taken at 100x and 200x using Leica Qwin Pro software. Additional experiment was performed including isotype IgG control raised in the secondary antibody host and images were taken at 200x and 640x magnification using Zen software on the Axiophot Zeiss microscope. Slides were reviewed blinded and independently by two observers for assessment of immunohistochemical staining. Per section 10 fields were scored using four predetermined categories (score $0=$ none, $1=$ mild, 2 = moderate, 3 = severe intensity). Four different compartments were chosen to evaluate intensity of staining: ${ }^{1)}$ proteinosis and intra-alveolar macrophages, ${ }^{2)}$ alveolar epithelial cells, ${ }^{3)}$ fibrosis in alveolar septa and ${ }^{4)}$ bronchial epithelium.

A Z-Score was calculated to score the relationship of each condition (DQ12 or DQ12-PVNO exposure) to the mean in a group of scores per time point. A Z -score of 0 means the score is the same as in the control (PBS) group. Z-scores per animal were calculated by averaging caspase-1 and IL-1 $\beta$ expression scores for all individually assessed compartments per time point and subsequently subtracting from each value the mean, $\mu$, of the respective PBS control value followed by dividing by the standard deviation $(\sigma)(\mathrm{Z}=(\mathrm{x}-\mu) / \sigma)$. Data from all animals were averaged and expressed as mean values $\pm \mathrm{SEM}$.

\section{Statistical analyses}

Data were analyzed by one-way analysis of variance (ANOVA) using the Student NeumanKeul's test to adjust for multiple pair-wise comparisons between treatment groups, or the Student's t- test where appropriate. The results from the histopathological scoring and IHC were evaluated using the nonparametric Mann-Whitney U-test. Differences with p-values < 0.05 were considered statistically significant. 


\section{RESULTS}

Crystalline silica polymorph-induced inflammasome readouts are associated with caspase-1 enzymatic activity in a surface reactivity dependent manner.

In order to compare inflammasome activation by different silica, we first determined comparative toxicity in BEAS-2B (Figure 1) and THP-1 cells (data not shown) using a range of equal surface area concentrations of DQ12 silica and cristobalite silica. Results in Figure 1 show that neither DQ12 nor cristobalite silica caused significant cell death at the lowest dose. At higher doses similar levels of cell death were observed for both polymorphs. Interestingly, PVNO-modified quartz at the highest dose did not elicit any toxicity. Comparative data were obtained in THP-1 cells. For further experiments silica concentrations of $150 \times 10^{6} \mu \mathrm{m}^{2} / \mathrm{cm}^{2}$ were used, allowing us to investigate the inflammatory and fibrogenic effects of these mineral particles as described previously [14,30].

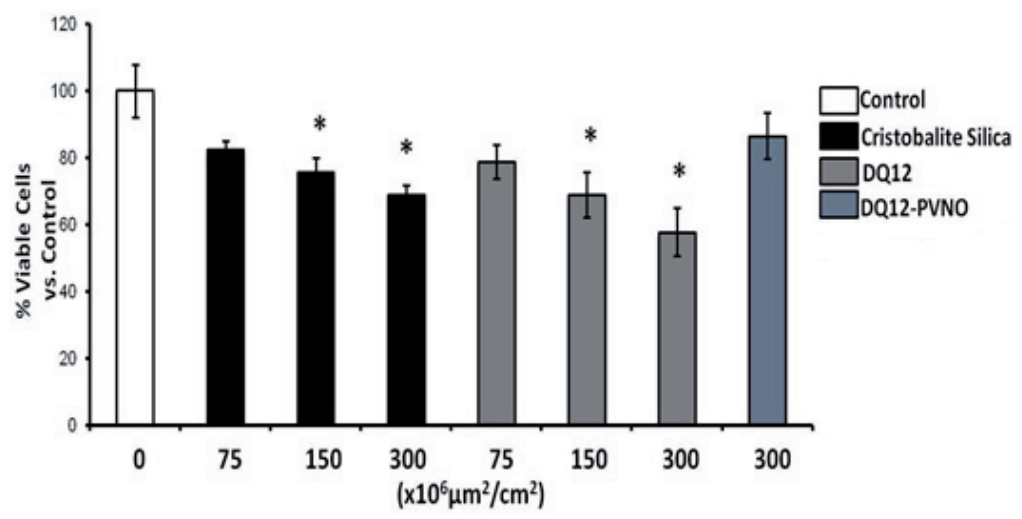

Figure 1: Assessment of BEAS-2B cell viability after exposure to different silica polymorphs. Cell viability assessed by the Trypan blue exclusion assay of cells exposed to indicated doses of cristobalite, DQ12 and DQ12-PVNO silica particles for $24 \mathrm{~h}$. Results are expressed as mean percent viable cells \pm SEM compared to unexposed controls (UC) and are representative of 3 independent experiments $(N=3$ for each treatment group in each experiment $) *$ denotes $p$-value $<0.05$ compared to controls.

We next assessed caspase-1 cleavage by both silica polymorphs comparatively at equal surface concentrations. Levels of the functional p20 subunit of caspase- 1 in BEAS-2B indeed similarly increased after treatment with cristobalite and DQ12 for $24 \mathrm{~h}$. Particle surface modification furthermore abrogated the potential of DQ12 to induce caspase-1 activation (Figure 2). 


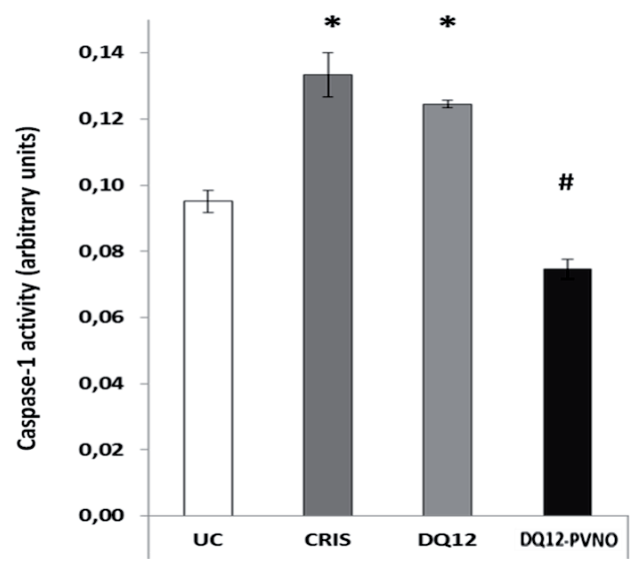

Figure 2: Silica polymorph-induced caspase-1 activity in human bronchial epithelial cells. Caspase1 enzymatic activity was assayed in lysates of BEAS-2B cells exposed to different silica polymorphs at a dose of $150 \times 10^{6} \mathrm{\mu m}^{2} / \mathrm{cm}^{2}$ for $24 \mathrm{~h}$. Caspase-1 activities are expressed as mean arbitrary units (O.D. $405 \mathrm{~nm} /$ total protein concentration $(\mathrm{mg} / \mathrm{mL})) \pm S E M$ with $* p$-value $<0.05$ compared to $U C$, and \# $p$ value $<0.05$ compared to DQ12.

We previously showed that release of not only IL-1 $\beta$, but also of bFGF and HMGB1 by BEAS-2B and THP-1 cells in response to cristobalite silica was dependent on NLRP3. Therefore we next assessed if DQ12 similarly caused the release of these inflammasomedependent mediators and if this then is dependent on surface reactivity as observed for caspase-1 activity in Figure 2. Indeed, results in Figure 3 demonstrate that cristobalite and DQ12 both act as inflammasome activators, causing significantly increased release of IL-1 $\beta$, bFGF and HMGB1 in both cell types. Levels of these mediators induced by both polymorphs were comparable in BEAS-2B cells. In THP-1 cells, bFGF and HMGB1 levels in response to cristobalite and DQ12 were similar as well, but DQ12 was found to be a more potent inducer of IL-1 $\beta$ release. The differential levels of these mediators observed between the two cell types under investigation, under baseline conditions as well as in response to silica treatment are in line with our previous study [14]. Interestingly, no significant increases in mediator levels in response to DQ12-PVNO compared to untreated controls were found. Also, compared to uncoated DQ12, the response was mostly significantly dampened by surface coating in both cell types. 

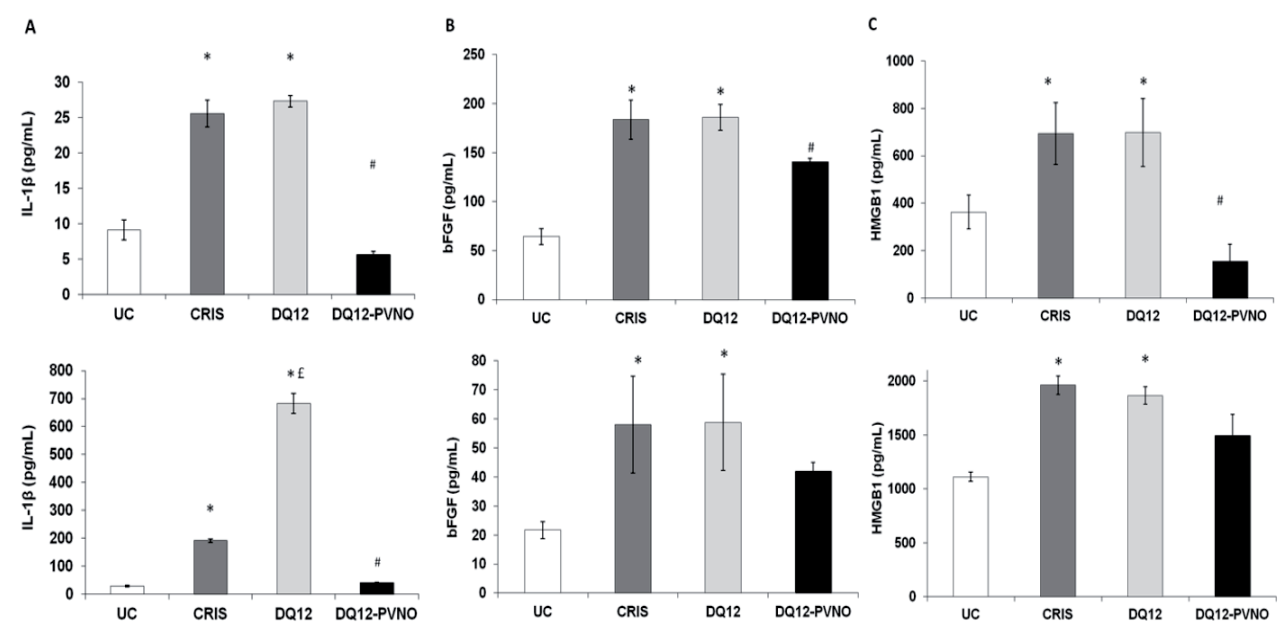

Figure 3: Silica polymorphs augment release of NLRP3-associated inflammatory and fibrotic mediators as well as alarmins from lung epithelial cells and differentiated macrophages, which is dependent on surface area reactivity. Investigation of markers for inflammasome activation in BEAS$2 B$ (upper panels) and THP-1 (lower panels) cells following exposure to cristobalite, native and PVNO coated DQ12 silica for $24 \mathrm{~h}$ at a dose of $150 \times 10^{6} \mathrm{\mu m}^{2} / \mathrm{cm}^{2}$. ELISAs were performed on concentrated medium for detection of secreted IL-1 $\beta$ (A), bFGF (B) and HMGB1 (C). Data are presented as mean \pm SEM with * p-value $<0.05$ compared to UC, $£$ p-value $<0.05$ compared to SIL150 and \# p-value $<0.05$ compared to DQ12 silica exposure.

Crystalline silica-induced caspase- 1 enzymatic activity and inflammasome readouts are attenuated by thioredoxin.

Intrinsic and extrinsic ROS formation is thought to be an essential step in the activation of the inflammasome in response to silica. Our data, with respect to dampening of the response by surface coating, support this hypothesis. One mechanism by which ROS are linked to inflammasome activation is by oxidation mediated release of TXNIP from TRX. We thus hypothesized that increasing TRX levels could prevent inflammasome activation in response to silica treatment. The presence of TRX was measured on WCL and concentrated SN of TRX plasmid transfected and TRX protein treated BEAS-2B cells by Western blotting (Additional file 2: Figure S1). In BEAS-2B (Figure 4A) as well as in THP-1 cells (Figure 4B), silica-induced caspase-1 activity levels were significantly lower in cells overexpressing TRX compared to pcDNA controls. In addition, pretreatment of BEAS-2B cells with recombinant human TRX protein similarly attenuated caspase-1 activation in response to silica treatment (data not shown). 

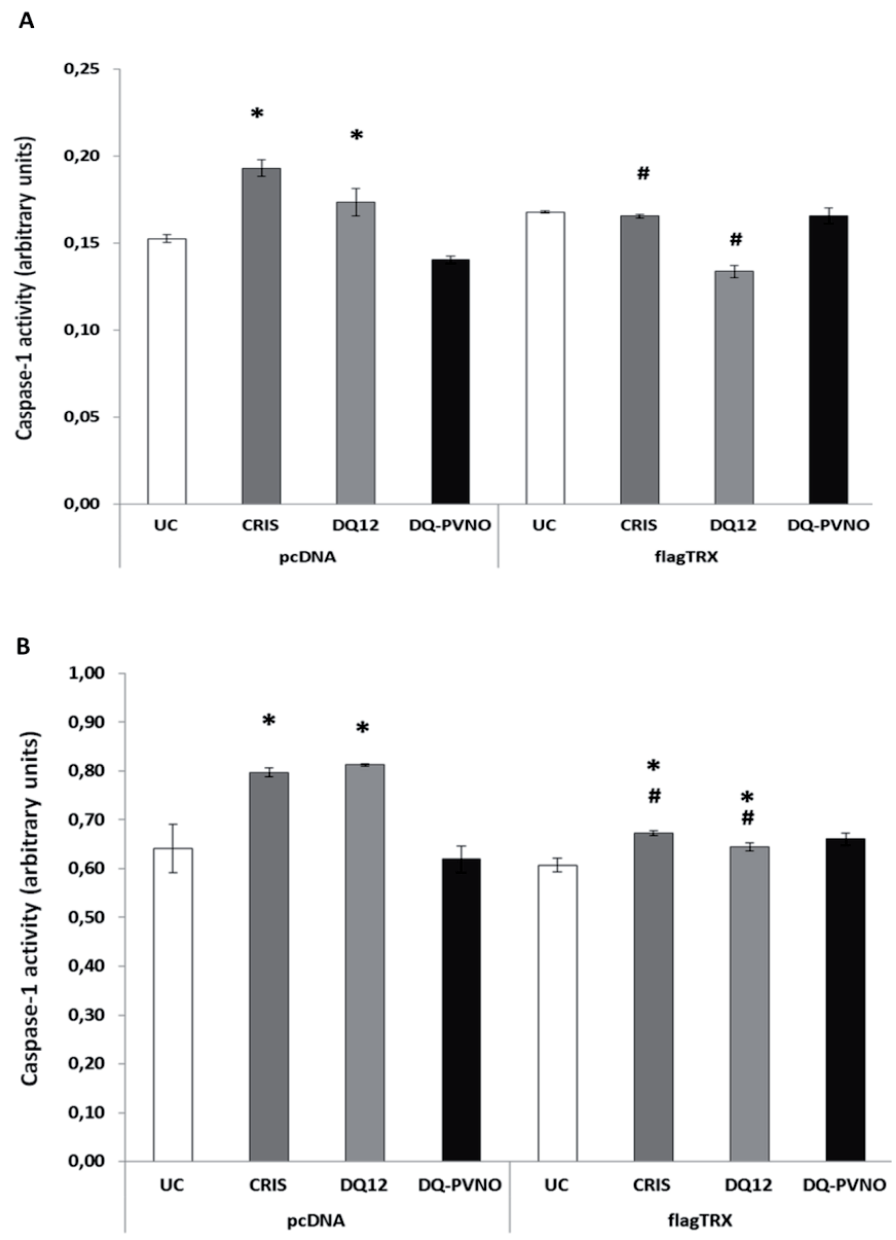

Figure 4: Silica-induced caspase-1 activity in human bronchial epithelial and macrophage-like cells is attenuated by thioredoxin. Caspase-1 enzymatic activity was assayed in cell lysates of BEAS$2 B$ (A) and THP-1 cells (B) exposed to different silica polymorphs for 24 at a dose of $150 x$ $10^{6} \mathrm{\mu m}^{2} / \mathrm{cm}^{2}$, transiently overexpressing flag-tagged thioredoxin (flagTRX) or pcDNA empty plasmid. Caspase-1 activities are expressed as mean arbitrary units (O.D. $405 \mathrm{~nm} /$ total protein concentration $(\mathrm{mg} / \mathrm{mL})) \pm S E M$ with * p-value $<0.05$ compared to appropriate controls $(U C)$, and \# $p$-value $<0.05$ compared to same treatment in pcDNA conditions.

To further investigate a protective role of TRX in crystalline silica-induced inflammasome signaling we determined IL-1 $\beta$ and HMGB1 levels in the medium of BEAS-2B cells overexpressing TRX or pretreated with recombinant human TRX protein (Figure 5). In agreement with effects observed on caspase-1 activity, both overexpression of TRX and pretreatment with recombinant TRX abrogated the release of IL-1 $\beta$ and HMGB1 by BEAS2B cells following cristobalite silica exposure. We also examined bFGF release in these experiments however these growth factor levels were not significantly affected by TRX (data 
not shown). Present results show that by enhancing TRX levels, either genetically or by treatment with the recombinant protein it is possible to attenuate inflammasome signaling.
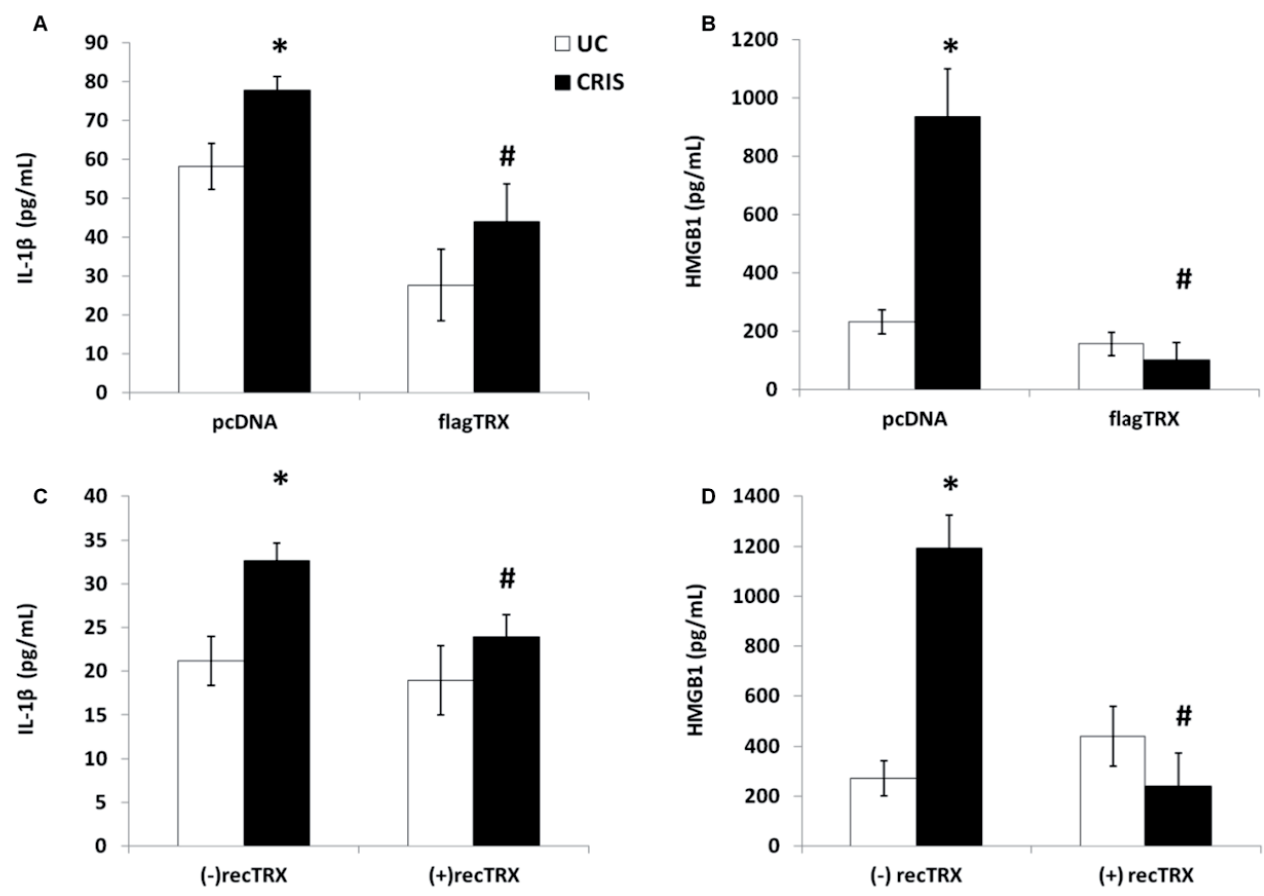

Figure 5: Thioredoxin overexpression or pretreatment attenuates the release of IL-1ß and HMGB1 in response to cristobalite silica. ELISAs were performed on concentrated medium of BEAS-2B cells transiently overexpressing flagTRX (top panel) or pre-treated with $250 \mathrm{ng} / \mathrm{ml}$ recombinant human $\operatorname{TRX}$ (lower panel) and exposed to $150 \times 10^{6} \mathrm{\mu m}^{2} / \mathrm{cm}^{2}$ of cristobalite silica for $24 \mathrm{~h}$ for the detection of secreted $I L-1 \beta$ ( $\boldsymbol{A}$ and $\boldsymbol{C}$ ) and HMGBI (B and D). Data are presented as mean $\pm S E M$ with * p-value $<0.05$ compared to $U C$, and \# p-value $<0.05$ compared to respective crystalline silica exposure in the pcDNA or non-TRX treated group.

Crystalline silica surface area reactivity is an important driver of lung damage, inflammation and histopathological hallmarks of silicosis in vivo.

As particle coating has been proven to affect in vivo responses as well with respect to inflammation and fibrosis, we examined how different cell types in rat lung tissue are affected over time when exposed to coated and uncoated crystalline minerals.

Representative lung tissue sections of rats exposed to PBS, coated and non-coated quartz for 90 days are shown in Figure 6. H\&E staining in the DQ12 exposed group demonstrated massive inflammation and particle-laden macrophages in the alveoli. Next to influx and accumulation of lymphocytes and neutrophils in the interstitium, alveolar histiocytosis accompanied with intra-alveolar proteinosis and hyperplasia was present in the native quartz group. Figure 6 also reveals the presence of fibrosis of the alveolar septa in lungs of rats exposed to DQ12 quartz. Quartz particles coated with PVNO did not cause disintegration of 
the alveolar structure. Moreover, DQ12-PVNO exposure revealed a decrease in the influx of inflammatory cells and an absence of fibrogenic histopathological characteristics in our silicosis model compared to uncoated quartz. The histopathological evaluations as evaluated by H\&E and Sirius Red staining and scoring thereof for all treatment times are listed in Table $\mathbf{1}$ and confirm the rapid pro-inflammatory effect of DQ12 as well as the progressive increases in pulmonary toxicity of this crystalline silica sample. By comparison, significantly less pronounced degrees of alveolar lipoproteinosis, alveolar histocytosis and bronchoalveolar hyperplasia were observed with the PVNO coated quartz with increasing time intervals. The surface modification of the DQ12 also led to lower fibrosis scores in the alveolar septa and perivascular regions.

\section{PBS}

DQ12

DQ12-PVNO

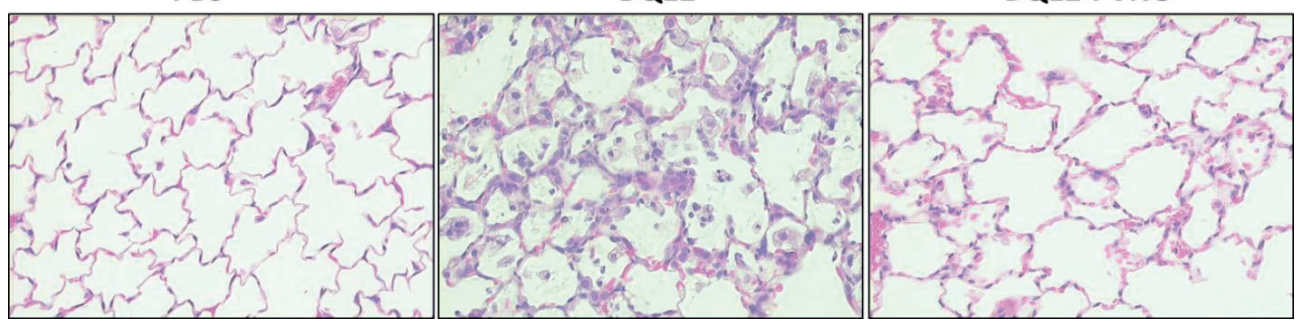

Figure 6: Particle surface modification attenuates the development of silicotic tissue morphology in rat lungs. Representative microphotographs of lung histopathology at 90 days after instillation of DQ12 or DQ12-PVNO by H\&E staining. Original magnification $\times 200$. 
Table 1: Summary of histopathological evaluation of lung tissue of rats exposed to DQ12, DQPVNO or PBS.

\begin{tabular}{clllllll}
\hline Alveolar lipoproteinosis & \multicolumn{5}{c}{ Mixed cell alveolar inflammation } \\
\hline & PBS & DQ12 & DQ12-PVNO & & PBS & DQ12 & DQ12-PVNO \\
\hline 3d & $0.0 \pm 0.0$ & $0.0 \pm 0.0$ & $0.0 \pm 0.0$ & 3d & $0.0 \pm 0.0$ & $1.2 \pm 0.7^{*}$ & $0.0 \pm 0.0^{\#}$ \\
$\mathbf{7 d}$ & $0.0 \pm 0.0$ & $0.2 \pm 0.4$ & $0.0 \pm 0.0$ & $\mathbf{7 d}$ & $0.0 \pm 0.0$ & $1.1 \pm 0.7^{*}$ & $0.5 \pm 0.6$ \\
$\mathbf{2 8 d}$ & $0.0 \pm 0.0$ & $1.0 \pm 0.3^{* *} 0.3 \pm 0.4^{\# \#}$ & $\mathbf{2 8 d}$ & $0.0 \pm 0.0$ & $0.8 \pm 1.1$ & $0.0 \pm 0.0$ \\
$\mathbf{9 0 d}$ & $0.0 \pm 0.0$ & $1.3 \pm 0.8^{*}$ & $0.0 \pm 0.0^{\#}$ & $\mathbf{9 0 d}$ & $0.0 \pm 0.0$ & $0.5 \pm 0.4$ & $0.1 \pm 0.2$ \\
$\mathbf{1 8 0 d}$ & $0.0 \pm 0.0$ & $2.0 \pm 0.4^{* *} 0.3 \pm 0.4^{\# \#}$ & $\mathbf{1 8 0 d}$ & $0.0 \pm 0.0$ & $0.0 \pm 0.0$ & $0.0 \pm 0.0$ \\
$\mathbf{3 6 0 d}$ & $0.0 \pm 0.0$ & $2.6 \pm 1.5^{* *} 2.9 \pm 1.5^{\$ \$}$ & $\mathbf{3 6 0 d}$ & $0.0 \pm 0.0$ & $0.6 \pm 1.0$ & $0.0 \pm 0.0$
\end{tabular}

Alveolar histocytosis

Brochoalveolar hyperplasia

$\begin{array}{clllclll} & \text { PBS } & \text { DQ12 } & \text { DQ12-PVNO } & & \text { PBS } & \text { DQ12 } & \text { DQ12-PVNO } \\ \text { 3d } & 0.0 \pm 0.0 & 0.8 \pm 0.7 & 0.1 \pm 0.3 & \text { 3d } & 0.0 \pm 0.0 & 0.7 \pm 0.9 & 0.3 \pm 0.7 \\ \text { 7d } & 0.3 \pm 0.7 & 1.5 \pm 0.7 & 0.5 \pm 0.4^{\#} & \mathbf{7 d} & 0.9 \pm 1.8 & 2.2 \pm 1.4 & 0.6 \pm 0.8 \\ \mathbf{2 8 d} & 1.0 \pm 0.4 & 3.4 \pm 1.4^{*} & 1.4 \pm 0.8^{\#} & \mathbf{2 8 d} & 1.0 \pm 0.4 & 3.1 \pm 1.8 & 1.4 \pm 1.2 \\ \mathbf{9 0 d} & 0.4 \pm 0.7 & 3.8 \pm 0.6^{* *} 1.0 \pm 0.7^{\# \#} & \mathbf{9 0 d} & 0.8 \pm 1.0 & 2.6 \pm 1.4 & 0.6 \pm 0.3 \\ \mathbf{1 8 0 d} & 0.8 \pm 0.4 & 2.4 \pm 0.6^{*} & 2.5 \pm 1.0^{\$} & \mathbf{1 8 0 d} & 0.1 \pm 0.3 & 2.3 \pm 1.0^{* *} & 2.3 \pm 0.7^{\$ S} \\ \mathbf{3 6 0 d} & 0.3 \pm 0.3 & 3.0 \pm 0.6^{* *} & 2.0 \pm 0.4^{\$ S, \#} & \mathbf{3 6 0 d} & 0.0 \pm 0.0 & 5.5 \pm 1.1^{* *} & 3.9 \pm 1.0^{\$ S, \#}\end{array}$

Fibrosis alveolar septa

Perivascular fibrosis

$\begin{array}{llllll}\text { PBS } & \text { DQ12 } & \text { DQ12-PVNO } & \text { PBS } & \text { DQ12 } & \text { DQ12-PVNO }\end{array}$

$\begin{array}{ccllclll}\mathbf{3 d} & 0.0 \pm 0.0 & 0.1 \pm 0.3 & 0.0 \pm 0.0 & \mathbf{3 d} & 1.0 \pm 0.2 & 1.0 \pm 0.0 & 0.9 \pm 0.2 \\ \mathbf{7 d} & 0.0 \pm 0.0 & 0.0 \pm 0.0 & 0.0 \pm 0.0 & \mathbf{7 d} & 0.6 \pm 0.2 & 1.0 \pm 0.0^{*} & 1.0 \pm 0.0 \\ \mathbf{2 8 d} & 0.0 \pm 0.0 & 0.4 \pm 0.6 & 0.0 \pm 0.0 & \mathbf{2 8 d} & 1.1 \pm 0.2 & 1.3 \pm 0.4 & 1.1 \pm 0.2 \\ \mathbf{9 0 d} & 0.0 \pm 0.0 & 1.0 \pm 0.8^{*} & 0.0 \pm 0.0^{\#} & \mathbf{9 0 d} & 1.0 \pm 0.0 & 1.4 \pm 0.4 & 1.0 \pm 0.0 \\ \mathbf{1 8 0 d} & 0.2 \pm 0.2 & 1.4 \pm 1.3 & 0.4 \pm 0.4 & \mathbf{1 8 0 d} & 0.8 \pm 0.2 & 1.6 \pm 0.2^{* *} & 1.5 \pm 0.4^{\S} \\ \mathbf{3 6 0 d} & 0.7 \pm 0.5 & 2.1 \pm 0.2^{* *} & 1.3 \pm 0.5^{\#} & \mathbf{3 6 0 d} & 1.1 \pm 0.4 & 2.3 \pm 0.2^{* *} & 1.9 \pm 0.4^{\$}\end{array}$

Histopathological evaluation of 2 lung lobes (left, right cranial) each stained with H\&E and Sirius red 3, 7, 28, 90, 180 and 360 days after one single intratracheal instillation of PBS, DQ12 or DQ12PVNO. For histopathological scoring, data were calculated as the sum of lesion severity and distribution. The mean of the two lobes was calculated and data expressed as mean \pm sd of 5 animals per treatment and time point. DQ12 vs. PBS: * $p<0.05 ; * * p<0.01 ; D Q 12-P V N O$ vs. PBS: ${ }^{\$} p<0.05$, ${ }^{s \$} p>0.01 ; D Q 12$ vs. DQ12-PVNO: \# $p<0.05$; \#\# $<<0.01$.

\section{Particle surface modification attenuates inflammasome activation in the BALF and rat lung tissue}

The contribution of inflammasome activation to the development of silicosis has been shown in a mouse model; however caspase-1 activity and its particle surface reactivity dependency in the BALF of rats was not demonstrated yet, neither has it been localized in an animal model of silicosis. In Figure 7 we showed increased levels of caspase-1 cleaved subunits in the BALF of DQ12 exposed rats. This effect was partly abrogated under conditions where rats were treated with the polymer-modified quartz. 


\section{3d}

$7 \mathbf{d}$

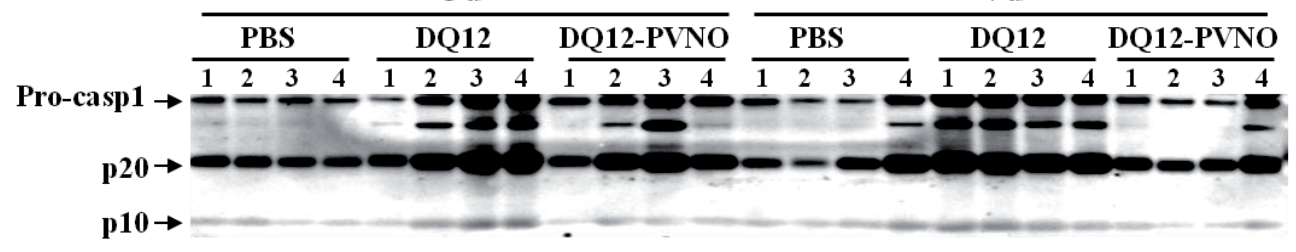

Figure 7: Detection of caspase-1 cleavage products in BALF of silica exposed rats. Cleaved caspase-1 20 and p10 subunits were detected in concentrated lavage fluid of rats exposed to PBS, DQ12 or DQ12-PVNO for 3 and 7 days by Western blotting (4 animals per group).

Another goal was to localize inflammasome activation in the lung tissue of the rat model used here and secondly to examine if this activation was dependent on surface reactivity. Immunohistochemistry for caspase-1 and IL- $1 \beta$ as pivotal readouts of inflammasome activation, to assess and localize activation of the inflammasome complex were performed. Clearly increased expression levels of caspase-1 and IL-1 $\beta$ in alveolar, bronchial epithelial, myeloid and endothelial cells in lungs of rats that were exposed to DQ12 for 180 days is evident in Figure 8. Additionally, exudates in alveolar proteinosis-characterized areas were significantly stained more intensely for these cleaved subunits and pro-forms of the protease and cytokine. A significant reduction in caspase- 1 and IL-1 $\beta$ staining was observed in lung tissue of the PVNO-coated rats. Immunohistochemical staining performed on lung tissue obtained at other time points under investigation reveal similar results (Additional file 3: Figure S2A-F). 

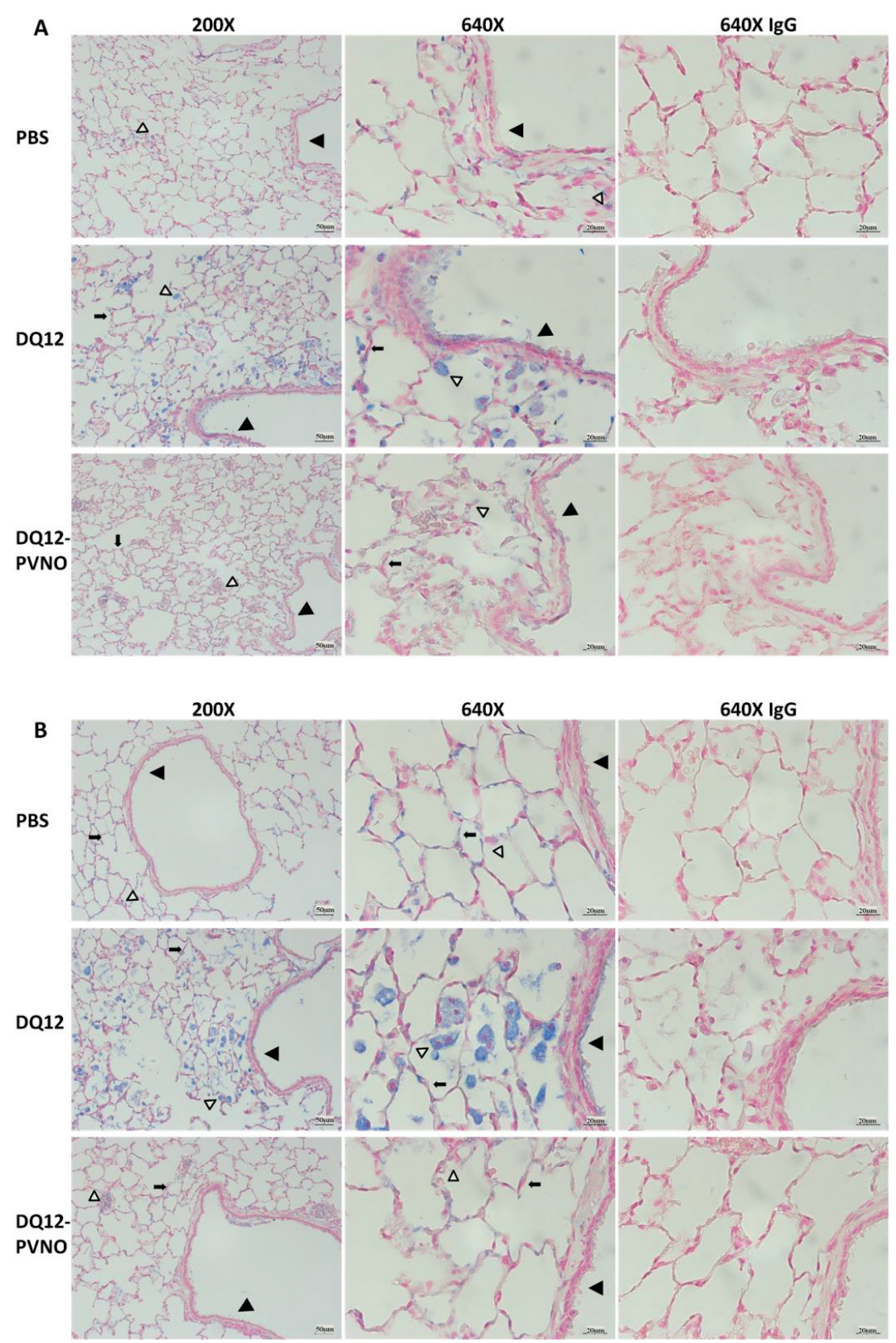

Figure 8: Particle surface modification modulates inflammasome activation in rat lungs. Immunohistochemical staining of caspase-1 (A) and IL-1B (B) in PBS control vs. DQ12 or DQ12-PVNO treated rats at 180 days after exposure along with corresponding $\operatorname{Ig} G$ control staining. Images are representative of 5 rats per group. Caspase- 1 and $I L-1 \beta$ are stained in blue. Slides were counterstained using Nuclear Fast Red. Arrows indicate specific cell types, i.e. bronchial epithelium (solid triangle), alveolar epithelial cell (arrow), macrophages (open triangle) 
Inflammasome activation, determined by caspase- 1 and IL-1 $\beta$ expression levels, was semiquantitatively scored, in different predefined compartments; ${ }^{1)}$ alveolar proteinosis and intraalveolar macrophages, ${ }^{2)}$ staining in alveolar epithelial cells, ${ }^{3)}$ inter-alveolar fibrosis and ${ }^{4)}$ bronchial epithelium. Figure 9A demonstrates that in each of the 4 compartments caspase-1 expression levels were significantly increased in the DQ12 treated group acutely and further increased at the later time points. The expression levels of caspase-1 were much lower at any time point in the polymer coated quartz exposed group compared to the native quartz exposed group. For day 3, 28, 180 similar data were obtained (not shown). Scoring of the expression of the pro-form and cleaved IL-1 $\beta$ subunits in these rat lung tissue sections provided data revealing similar patterns regarding inflammasome activation by crystalline silica including time-dependent increases and blunting by surface modified quartz exposure (Figure 9B). Finally, an overall score for inflammasome activation was calculated based on these two immunohistochemical stainings individually assessed in the 4 separate compartments per time point for each quartz treatment in relation to the PBS controls (Figure 9C). A Z-score of 0 indicates that the overall inflammasome score in that specific condition does not differ from the mean score of the control group. DQ12 exposure thus induced inflammasome activation at all time points, and this level of inflammasome activation was largely attenuated in the uncoated quartz group. 

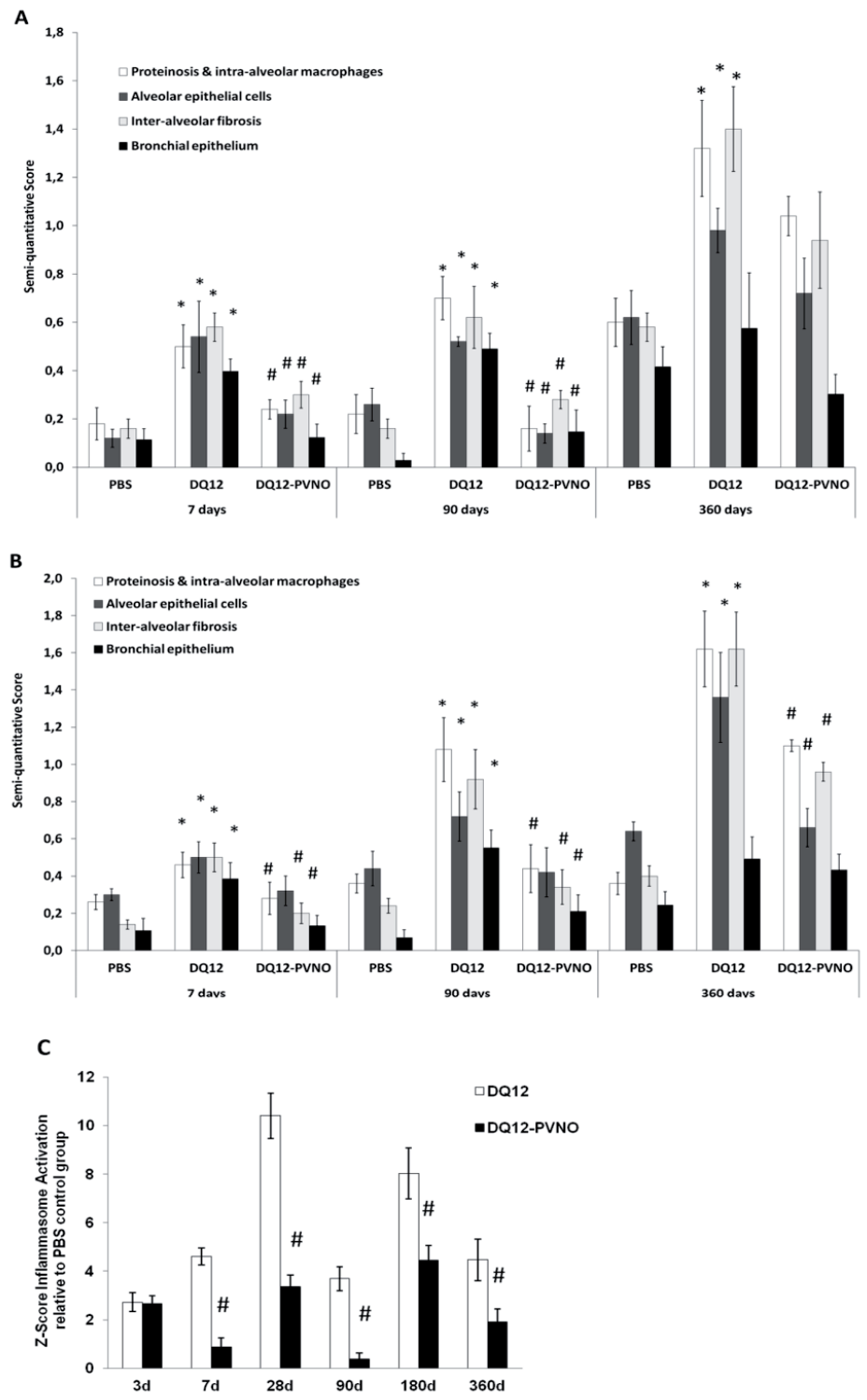

Figure 9: Native quartz exposure leads to sustained inflammasome activation in different cellular and interstitial compartments of the rat lung, which is attenuated by surface modification. Results represent semi-quantitative scoring of caspase-1 (A) and IL-1 $\beta$ (B) immunohistochemistry in different compartments of lungs at acute (7 days) and chronic inflammatory conditions (90 and 360 days) following a single silica or PBS challenge. Data are presented as mean $\pm S E M$ with * p-value $<0.05$ compared to PBS, and \# p-value $<0.05$ compared to DQ12 exposure. C, Z-scores for total inflammasome (of caspase-1 and IL-1 $\beta$ combined) staining intensities over all compartments in DQ12 and DQ12-PVNO groups normalized to the average inflammasome staining of the PBS group at each time point with \# p-value $<0.05$ comparing DQ12-PVNO vs UC with DQ12 vs UC. 


\section{DISCUSSION}

Prolonged exposure to silica can lead to the development of silicosis, an irreversible, fibrotic pulmonary disease. Inflammasome activation by silica has been reported in myeloid and epithelial cells of the lung in vitro and is crucial to development of silicosis in mice [6,13]. In this study we demonstrated that the inflammasome pathway is activated in inflammatory as well as structural cells in rat lungs up to 1 year after a single intratracheal instillation of crystalline silica in association with pulmonary inflammation and fibrogenesis. Further, we found these effects to be largely absent when PVNO -polymer modified quartz was instilled, indicating the importance of the surface modification in vivo to both the induction of inflammasome activation as well as associated inflammatory and fibrotic responses. It is of notice that the PVNO-coated material has lower effects although, especially at later time points post instillation, some effects are emerging (180 and 360 days). This could perhaps be due to the removal of some of the coating over time. In fact the data show that PVNO-coating blunts much of the toxic responses of the DQ12, however, it does not turn the DQ12 into an "inert" dust.

Secondly, in epithelial and myeloid cells inflammasome activation was demonstrated in vitro to a similar extent by different silica polymorphs, which was also found to be critically dependent on surface reactivity of the crystalline dust. Additionally, inflammasomedependent mediator release could be reduced not only by surface modification, but also by enhancing endogenous thioredoxin levels by overexpression or exogenous administration of this antioxidant.

In the in vivo study increased activation of caspase-1 and increased levels of IL-1 $\beta$ were observed in response to DQ12 treatment in different cellular compartments of the lung, at acute, as well as chronic timepoints. Here, pro- as well as cleaved/active subunits of these proteins, so the total amount of this protein is detected in the rat lung tissues. However, it has been shown that when activated, increased mRNA expression of both capase-1 and IL-1 $\beta$ occurs as a positive feedback mechanism to replenish protein levels and provide sufficient substrate. The increased protein levels of caspase-1 and IL-1 $\beta$ although not specific for activated forms, can be regarded as proof of activation of the inflammasome. These data indicate a synergistic contribution of alveolar and bronchial epithelial cells as well as myeloid cells, endothelial cells and fibroblasts to the total inflammasome activation which has been reported previously in mouse models of silicosis.

In vitro studies have revealed that, in response to silica and other crystals, immune cells deficient in components of the NLRP3 inflammasome are incapable of secreting the proinflammatory cytokines IL-1 $\beta$ and IL-18 [7,14,31]. Silica-induced inflammasome activation in non-myeloid cells is emphasized in recent publications where interestingly human keratinocytes released mature, cleaved IL-1 $\beta$ only upon exposure to $\mathrm{SiO} 2$ nanoparticles, although it has not been fully characterized to what extent nano-SiO2 is crystalline [32]. Our group furthermore demonstrated the presence and functional activation of the NLRP3 inflammasome in human lung epithelial cells in response to cristobalite silica [14]. By the studies represented in this manuscript we add to these data that inflammasome activation in bronchial epithelial cells and differentiated macrophages can be induced to a similar degree by the two most common polymorphs of crystalline silica, i.e. quartz and 
cristobalite, to secrete IL-1 $\beta$, bFGF and HMGB1 from human lung cells in vitro. Accordingly, from a review of in vivo experimental and epidemiological studies, Mossman and Glenn recently synthesized no evidence for differences between quartz and cristobalite in being proinflammatory and possibly fibrogenic agents [33]. Besides silica, also asbestos fibers (hydrated silicates) can prime and activate the NLRP3 inflammasome [5,34] in for instance human mesothelial cells [35]. The importance of the NLRP3 inflammasome and HMGB1 in asbestos-induced inflammation has in addition been linked to mesothelioma [36].

The polymer PVNO has been investigated for its potential prophylactic and therapeutic use in silica and silica-containing dust induced fibrosis, albeit with limited success [37-39]. Next to addition, adsorption of PVNO onto the crystalline silica surface has been shown to blunt its reactivity via a mechanism that is considered to involve H-bonding of its NO groups with the reactive silanol groups at the quartz surface. A direct coating approach of quartz with the polymer has been used by us in various investigations to address the contribution of the surface properties in silica toxicity and pathogenesis [24,25]. First, it could be demonstrated by electron spin resonance spectroscopy that PVNO coating inhibits the intrinsic ROS properties of DQ12 quartz. Subsequently, it was shown that such modification abrogates ROS-mediated induction of oxidative DNA damage responses in lung epithelial cells in vitro $[25,40]$ and rat lungs in vivo [24]. PVNO coating has also specifically been shown to abrogate the formation of ROS by phagocytic cells and ROS levels in lavage fluid of exposed rats $[20,24]$. In association with this, PVNO modification also had profound effects on the induction and persistence of pulmonary inflammation [21] as well as the cellular uptake of silica particles and their clearance from rat lung [41]. This is further evidenced by our additional proof for in vivo surface reactivity dependent inflammasome activation by means of caspase-1 activity in the BALF. Our present data in addition reveal an important role for the crystalline surface of silica polymorphs in the activation of caspase- 1 and in the ability to induce secretion of IL-1 $\beta$, bFGF and HMGB1 from human lung epithelial and macrophage like cells in vitro and in inflammasome activation in vivo.

Activation of the NLRP3 inflammasome by silica requires both an efflux of intracellular potassium and the generation of reactive oxygen species in myeloid cells [6]. Hornung et al. added evidence that particle uptake in peripheral blood mononuclear cells subsequently leads to lysosomal damage and rupture, and that inhibition of either phagosomal acidification or cathepsin B activity impaired NLRP3 activation. Their work indicates that the NLRP3 inflammasome senses lysosomal damage as an endogenous 'danger' signal [7]. We previously demonstrated impaired inflammasome activation upon inhibition of uptake by cytochalasin D pretreatment in epithelial cells [14]. Uptake of silica polymorphs, NADPH oxidase activity and ROS formation has also been linked to NLRP3 inflammasome activation in THP-1 macrophages and bone marrow-derived dendritic cells [5,31]. Shifts in the intracellular redox status resulting from an imbalance between ROS formation and antioxidant defense pathways are known to modulate innate immunity at various levels in myeloid and epithelial cells. Therefore, as expected, abrogated inflammasome signals were detected when BEAS-2B or THP-1 cells were exposed to aforementioned surface modified quartz. This was evidenced by attenuated release of IL-1 $\beta$, bFGF and HMGB1, in addition to lower induction of caspase-1 activity by PVNO surface modification of DQ12 and supported by our in vivo findings. These 
data indicate that oxidant imbalances arising from particle-surface interactions may be responsible for the earlier described inflammasome activation. Also in relation to the involvement of oxidant imbalances in inflammasome activation, a key role for the thioredoxin-interacting protein (TXNIP)/TRX axis, recently coined as the "redoxisome" [42] has been evidenced [16]. Thioredoxins (TRX) reduce oxidized proteins and regulate the function of several proteins by acting as a binding partner. TRX has been for instance been described as potent ROS detoxifying protein in retinal pigment epithelial cells and has a direct effect on mitochondria by preventing oxidative stress [43]. Dostert et al. devoted a role to TRX in regulating inflammasome activation in THP1-macrophages in response to asbestos and uric acid crystals [5]. More recent work by Zhou et al. furthermore showed that lysosomal damage or increased oxidant production as a consequence of particle-cell interactions and uptake, would lead to increased dissociation of TXNIP from TRX, which was essential to inflammasome activation. Additionally, it was shown that asbestos modulates TRX/TXNIP interaction to regulate inflammasome activation [17]. We thus hypothesized that the described absence of inflammasome activation in TXNIP deficient conditions could be mimicked by increasing TRX levels. Indeed, overexpression or pretreatment with TRX1 significantly attenuated caspase- 1 activation and release of IL-1 $\beta$ and HMGB1 in response to silica treatment. Interestingly, in previous studies we had observed significantly increased mRNA levels of TXNIP in primary normal human bronchial epithelial cells after cristobalite silica treatment [30] and confirmed cristobalite-induced increased protein levels in BEAS-2B by Western blot analysis (data not shown). These novel data suggest that the inflammasome readouts we observed are associated with caspase-1 enzyme activity in a thioredoxin dependent fashion in vitro. In conjunction with reported protection to induction of inflammation and airway hyperresponsiveness in a mouse model of asthma and development of emphysema and ongoing inflammation in a model of COPD by intraperitoneal injection of recombinant TRX $[44,45]$, further investigations towards the possible therapeutic potential of TRX in silicosis and diseases characterized by inflammasome activation in general are warranted.

Taken together, these results suggest that quartz and cristobalite silica can induce inflammasome activation in vitro and further that exposure to most abundant crystalline polymorph, quartz, in vivo is associated with inflammasome-dependent inflammatory and fibrotic remodelling of lung tissue in a surface reactivity dependent manner. Although the current study provides evidence for inflammasome activation in structural cells in addition to myeloid cells, the relative importance of inflammasome activation in these different cell types in relation to the development and progression of silicosis needs to be further investigated. 


\section{REFERENCES}

1. Laney AS, Petsonk EL, Hale JM, Wolfe AL, Attfield MD: Potential determinants of coal workers' pneumoconiosis, advanced pneumoconiosis, and progressive massive fibrosis among underground coal miners in the United States, 2005-2009. Am J Public Health 2012, 102 Suppl 2:S279-283.

2. Adverse effects of crystalline silica exposure. American Thoracic Society Committee of the Scientific Assembly on Environmental and Occupational Health. American journal of respiratory and critical care medicine 1997, 155:761-768.

3. Mossman BT, Churg A: Mechanisms in the pathogenesis of asbestosis and silicosis. American journal of respiratory and critical care medicine 1998, 157:1666-1680.

4. Hessel PA, Gamble JF, Gee JB, Gibbs G, Green FH, Morgan WK, Mossman BT: Silica, silicosis, and lung cancer: a response to a recent working group report. Journal of occupational and environmental medicine / American College of Occupational and Environmental Medicine 2000, 42:704-720.

5. Dostert C, Petrilli V, Van Bruggen R, Steele C, Mossman BT, Tschopp J: Innate immune activation through Nalp3 inflammasome sensing of asbestos and silica. Science 2008, 320:674677.

6. Cassel SL, Eisenbarth SC, Iyer SS, Sadler JJ, Colegio OR, Tephly LA, Carter AB, Rothman PB, Flavell RA, Sutterwala FS: The Nalp3 inflammasome is essential for the development of silicosis. Proceedings of the National Academy of Sciences of the United States of America 2008, 105:9035-9040.

7. Hornung V, Bauernfeind F, Halle A, Samstad EO, Kono H, Rock KL, Fitzgerald KA, Latz E: Silica crystals and aluminum salts activate the NALP3 inflammasome through phagosomal destabilization. Nature immunology 2008, 9:847-856.

8. Martinon F, Burns K, Tschopp J: The inflammasome: a molecular platform triggering activation of inflammatory caspases and processing of proIL-beta. Mol Cell 2002, 10:417-426.

9. Keller M, Ruegg A, Werner S, Beer HD: Active caspase-1 is a regulator of unconventional protein secretion. Cell 2008, 132:818-831.

10. Lamkanfi M, Sarkar A, Vande Walle L, Vitari AC, Amer AO, Wewers MD, Tracey KJ, Kanneganti TD, Dixit VM: Inflammasome-dependent release of the alarmin HMGB1 in endotoxemia. J Immunol 2010, 185:4385-4392.

11. Rubartelli A: Redox control of NLRP3 inflammasome activation in health and disease. $J$ Leukoc Biol 2012, 92:951-958.

12. Piguet PF, Vesin C, Grau GE, Thompson RC: Interleukin 1 receptor antagonist (IL-1ra) prevents or cures pulmonary fibrosis elicited in mice by bleomycin or silica. Cytokine 1993, 5:57-61.

13. Guo J, Gu N, Chen J, Shi T, Zhou Y, Rong Y, Zhou T, Yang W, Cui X, Chen W: Neutralization of interleukin-1 beta attenuates silica-induced lung inflammation and fibrosis in C57BL/6 mice. Arch Toxicol 2013, 87:1963-1973.

14. Peeters PM, Perkins TN, Wouters EF, Mossman BT, Reynaert NL: Silica induces NLRP3 inflammasome activation in human lung epithelial cells. Particle and fibre toxicology 2013, 10:3. 
15. Halle A, Hornung V, Petzold GC, Stewart CR, Monks BG, Reinheckel T, Fitzgerald KA, Latz E, Moore KJ, Golenbock DT: The NALP3 inflammasome is involved in the innate immune response to amyloid-beta. Nat Immunol 2008, 9:857-865.

16. Zhou R, Tardivel A, Thorens B, Choi I, Tschopp J: Thioredoxin-interacting protein links oxidative stress to inflammasome activation. Nat Immunol 2010, 11:136-140.

17. Thompson JK, Westbom CM, MacPherson MB, Mossman BT, Heintz NH, Spiess P, Shukla A: Asbestos modulates thioredoxin-thioredoxin interacting protein interaction to regulate inflammasome activation. Part Fibre Toxicol 2014, 11:24.

18. Donaldson K, Borm PJ: The quartz hazard: a variable entity. Ann Occup Hyg 1998, 42:287-294.

19. Fubini B: Surface reactivity in the pathogenic response to particulates. Environ Health Perspect 1997, 105 Suppl 5:1013-1020.

20. Albrecht C, Knaapen AM, Becker A, Hohr D, Haberzettl P, van Schooten FJ, Borm PJ, Schins RP: The crucial role of particle surface reactivity in respirable quartz-induced reactive oxygen/nitrogen species formation and APE/Ref-1 induction in rat lung. Respir Res 2005, 6:129.

21. Albrecht C, Schins RP, Hohr D, Becker A, Shi T, Knaapen AM, Borm PJ: Inflammatory time course after quartz instillation: role of tumor necrosis factor-alpha and particle surface. $\mathrm{Am} J$ Respir Cell Mol Biol 2004, 31:292-301.

22. Donaldson K, Stone V, Duffin R, Clouter A, Schins R, Borm P: The quartz hazard: effects of surface and matrix on inflammogenic activity. J Environ Pathol Toxicol Oncol 2001, 20 Suppl 1:109-118.

23. Duffin R, Gilmour PS, Schins RP, Clouter A, Guy K, Brown DM, MacNee W, Borm PJ, Donaldson K, Stone V: Aluminium lactate treatment of DQ12 quartz inhibits its ability to cause inflammation, chemokine expression, and nuclear factor-kappaB activation. Toxicol Appl Pharmacol 2001, 176:10-17.

24. Knaapen AM, Albrecht C, Becker A, Hohr D, Winzer A, Haenen GR, Borm PJ, Schins RP: DNA damage in lung epithelial cells isolated from rats exposed to quartz: role of surface reactivity and neutrophilic inflammation. Carcinogenesis 2002, 23:1111-1120.

25. Schins RP, Duffin R, Hohr D, Knaapen AM, Shi T, Weishaupt C, Stone V, Donaldson K, Borm PJ: Surface modification of quartz inhibits toxicity, particle uptake, and oxidative DNA damage in human lung epithelial cells. Chem Res Toxicol 2002, 15:1166-1173.

26. Hillegass JM, Shukla A, Lathrop SA, MacPherson MB, Fukagawa NK, Mossman BT: Assessing nanotoxicity in cells in vitro. Wiley Interdiscip Rev Nanomed Nanobiotechnol 2010, 2:219-231.

27. Haendeler J, Hoffmann J, Tischler V, Berk BC, Zeiher AM, Dimmeler S: Redox regulatory and anti-apoptotic functions of thioredoxin depend on S-nitrosylation at cysteine 69. Nat Cell Biol 2002, 4:743-749.

28. Hohr D, Steinfartz Y, Schins RP, Knaapen AM, Martra G, Fubini B, Borm PJ: The surface area rather than the surface coating determines the acute inflammatory response after instillation of fine and ultrafine $\mathrm{TiO} 2$ in the rat. Int J Hyg Environ Health 2002, 205:239-244.

29. Porter DW, Ramsey D, Hubbs AF, Battelli L, Ma J, Barger M, Landsittel D, Robinson VA, McLaurin J, Khan A, Jones W, Teass A, Castranova V: Time course of pulmonary response of 
rats to inhalation of crystalline silica: histological results and biochemical indices of damage, lipidosis, and fibrosis. J Environ Pathol Toxicol Oncol 2001, 20 Suppl 1:1-14.

30. Perkins TN, Shukla A, Peeters PM, Steinbacher JL, Landry CC, Lathrop SA, Steele C, Reynaert NL, Wouters EF, Mossman BT: Differences in gene expression and cytokine production by crystalline vs. amorphous silica in human lung epithelial cells. Particle and fibre toxicology 2012, 9:6.

31. Winter M, Beer HD, Hornung V, Kramer U, Schins RP, Forster I: Activation of the inflammasome by amorphous silica and $\mathrm{TiO} 2$ nanoparticles in murine dendritic cells. Nanotoxicology 2011, 5:326-340.

32. Yazdi AS, Guarda G, Riteau N, Drexler SK, Tardivel A, Couillin I, Tschopp J: Nanoparticles activate the NLR pyrin domain containing 3 (Nlrp3) inflammasome and cause pulmonary inflammation through release of IL-1alpha and IL-1beta. Proceedings of the National Academy of Sciences of the United States of America 2010, 107:19449-19454.

33. Mossman BT, Glenn RE: Bioreactivity of the crystalline silica polymorphs, quartz and cristobalite, and implications for occupational exposure limits (OELs). Critical reviews in toxicology 2013, 43:632-660.

34. Li M, Gunter ME, Fukagawa NK: Differential activation of the inflammasome in THP-1 cells exposed to chrysotile asbestos and Libby "six-mix" amphiboles and subsequent activation of BEAS-2B cells. Cytokine 2012, 60:718-730.

35. Hillegass JM, Miller JM, MacPherson MB, Westbom CM, Sayan M, Thompson JK, Macura SL, Perkins TN, Beuschel SL, Alexeeva V, Pass HI, Steele C, Mossman BT, Shukla A: Asbestos and erionite prime and activate the NLRP3 inflammasome that stimulates autocrine cytokine release in human mesothelial cells. Part Fibre Toxicol 2013, 10:39.

36. Carbone M, Yang H: Molecular pathways: targeting mechanisms of asbestos and erionite carcinogenesis in mesothelioma. Clinical cancer research: an official journal of the American Association for Cancer Research 2012, 18:598-604.

37. Goldstein B, Rendall RE: The prophylactic use of polyvinylpyridine-N-oxide (PVNO) in baboons exposed to quartz dust. Environ Res 1987, 42:469-481.

38. Hilscher W, Schlipkoter HW: Antagonistic factors in the pathogenesis of coal workers' pneumoconiosis. Ann N Y Acad Sci 1972, 200:166-181.

39. Weller W: Long-term test on rhesus monkeys for the PVNO therapy of anthracosilicosis. Inhaled Part 1975, 4 Pt 1:379-387.

40. Schins RP, Knaapen AM, Cakmak GD, Shi T, Weishaupt C, Borm PJ: Oxidant-induced DNA damage by quartz in alveolar epithelial cells. Mutat Res 2002, 517:77-86.

41. Albrecht C, Hohr D, Haberzettl P, Becker A, Borm PJ, Schins RP: Surface-dependent quartz uptake by macrophages: potential role in pulmonary inflammation and lung clearance. Inhalation toxicology 2007, 19 Suppl 1:39-48.

42. Yoshihara E, Masaki S, Matsuo Y, Chen Z, Tian H, Yodoi J: Thioredoxin/Txnip: Redoxisome, as a Redox Switch for the Pathogenesis of Diseases. Front Immunol 2014, 4:514. 
43. Sugano E, Isago $\mathrm{H}$, Murayama $\mathrm{N}$, Tamai $\mathrm{M}$, Tomita $\mathrm{H}$ : Different anti-oxidant effects of thioredoxin 1 and thioredoxin 2 in retinal epithelial cells. Cell structure and function 2013, 38:8188 .

44. Kinoshita T, Hoshino T, Imaoka H, Ichiki H, Okamoto M, Kawayama T, Yodoi J, Kato S, Aizawa H: Thioredoxin prevents the development and progression of elastase-induced emphysema. Biochem Biophys Res Commun 2007, 354:712-719.

45. Nakamura H, Hoshino Y, Okuyama H, Matsuo Y, Yodoi J: Thioredoxin 1 delivery as new therapeutics. Adv Drug Deliv Rev 2009, 61:303-309. 


\section{SUPPLEMENTAL MATERIAL}

Additional file 1: Table S1 Histopathological scoring was performed according to schematic represented in Additional file 3: Table S1 [29].

\begin{tabular}{|l|l|l|l|l|}
\hline Severity & No & Focal & Multifocal & Locally extensive \\
\hline 1 & 1.0 & 0.25 & 0.5 & 0.75 \\
\hline 2 & 2.0 & 1.25 & 1.5 & 1.75 \\
\hline 3 & 3.0 & 2.25 & 2.5 & 2.75 \\
\hline 4 & 4.0 & 3.25 & 3.5 & 3.75 \\
\hline
\end{tabular}

Additional file 2: Figure S1 Determination of TRX in BEAS-2B cells that were transferred with TRX plasmid or treated with recombinant TRX protein. BEAS-2B cells were transfected or treated with TRX plasmid or TRX protein, respectively. On whole cell lysates (WCL) and concentrated supernatants (SN) the presence of TRX was measured by western blot. GAPDH (1:20000) was used as an house keeping control.

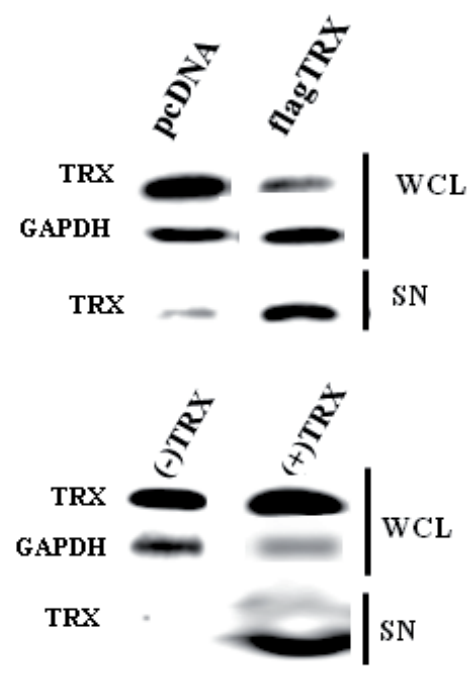

Additional file 3: Figure S2A-F IHC images of caspase-1 and IL-1 $\beta$ at given time points. These supplemental figures represent immunohistochemical staining for caspase-1 on lung tissue obtained at 3 and 7 days (2A), at 28 and 90 days (2B) as well as at 180 and 360 days (2C). Representative images for IL-1 $\beta$ staining are indicated for different timepoints: 3 and 7 days (2D), at 28 and 90 days (2E) as well as at 180 and 360 days (2F). 
2A
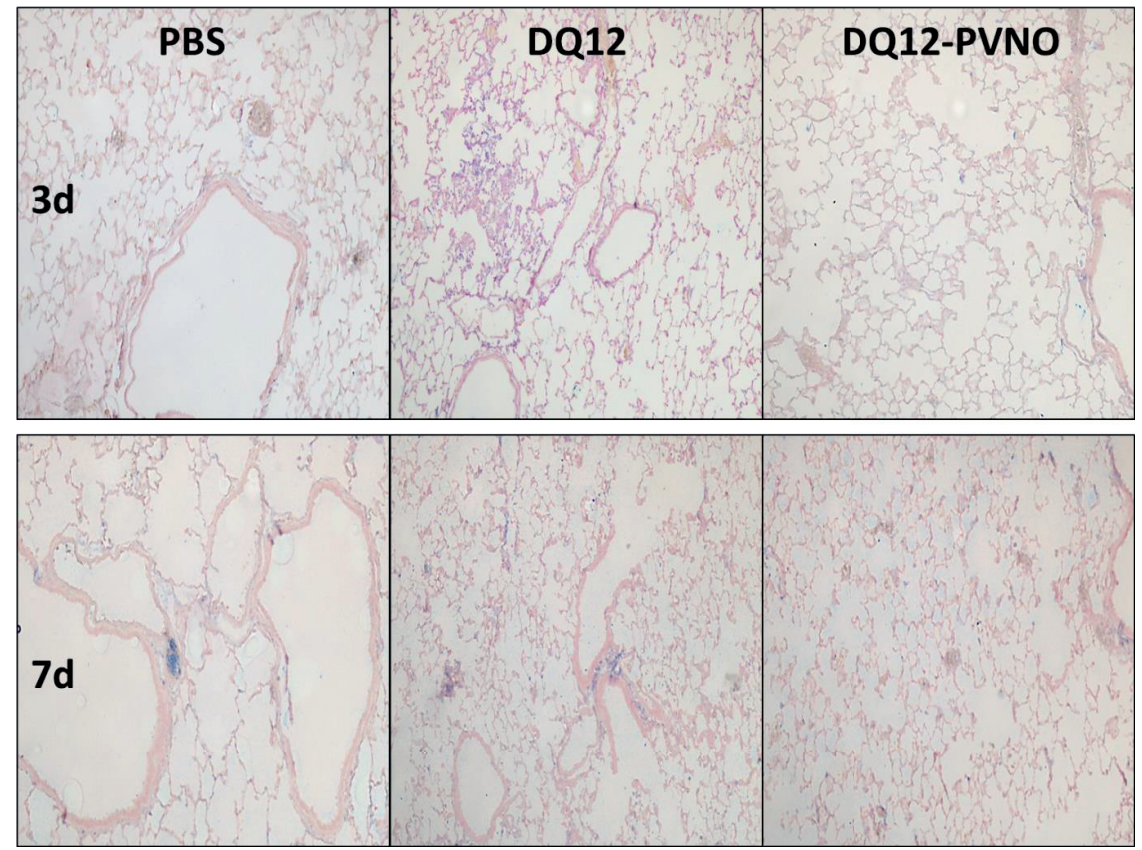

2B
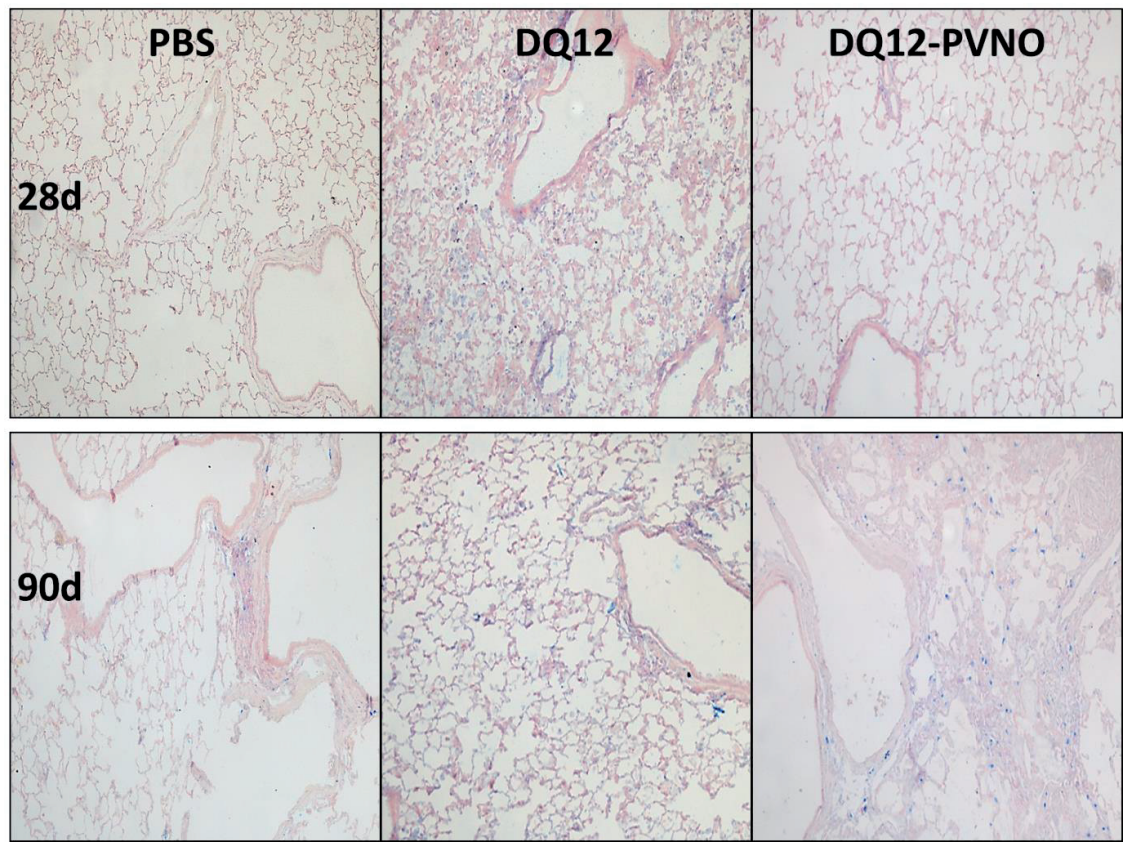
2C
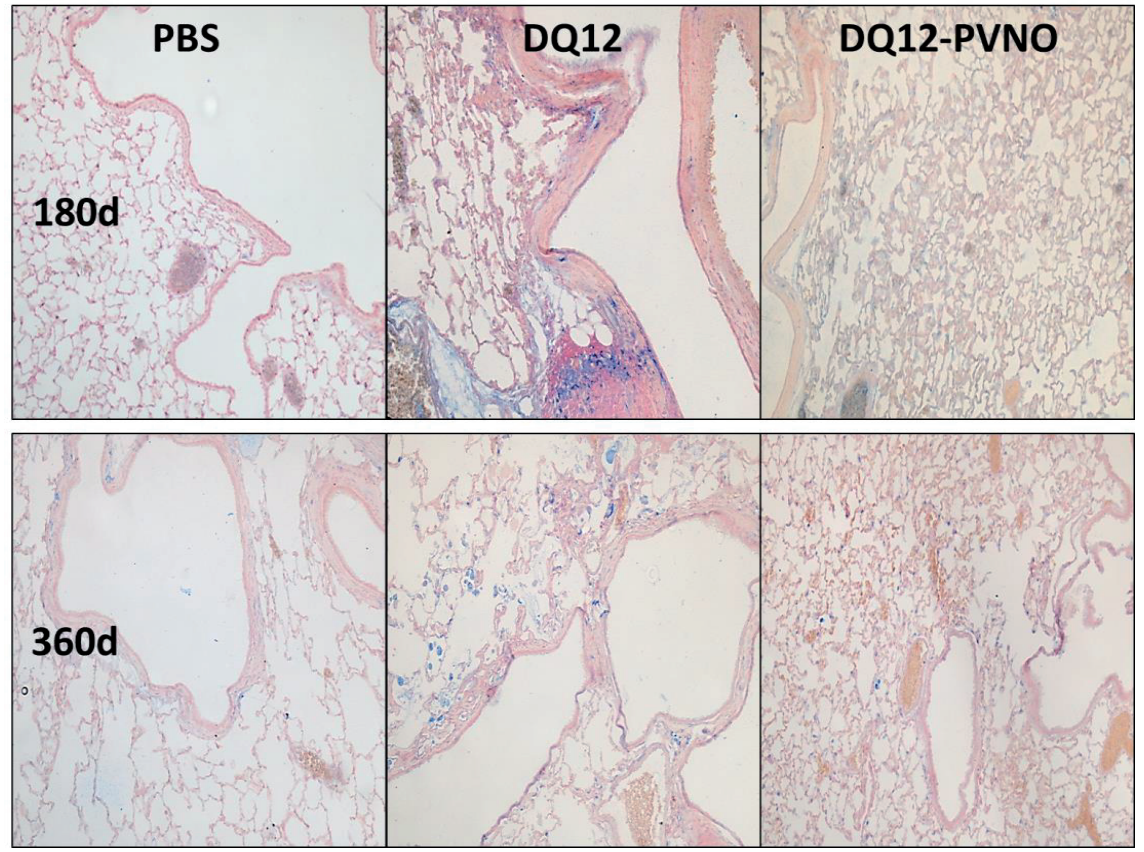

2D
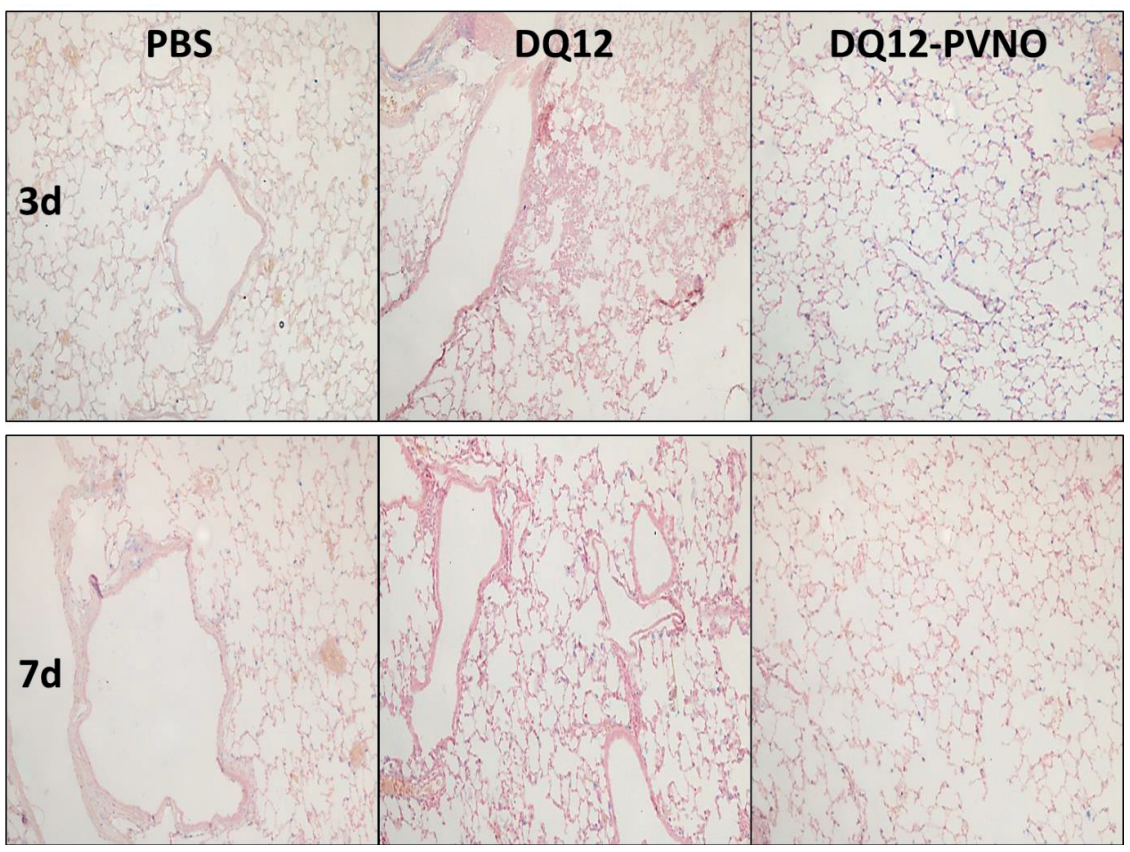
2E
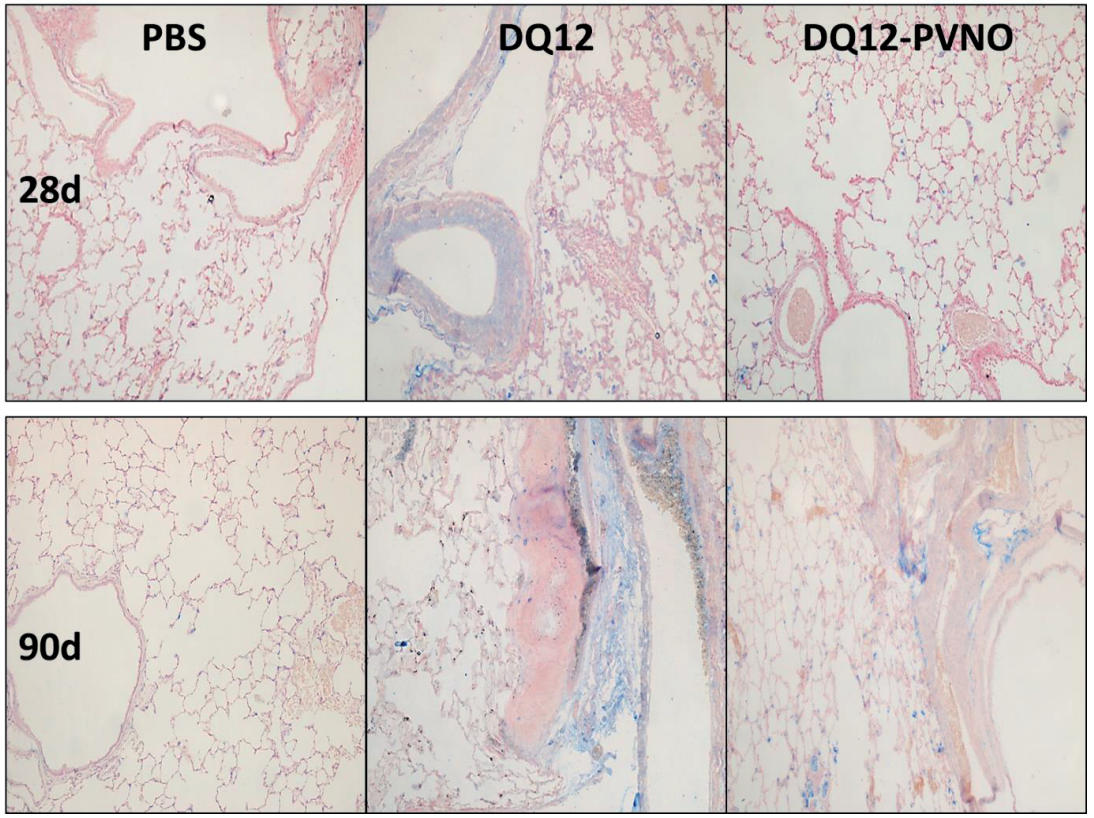

2F
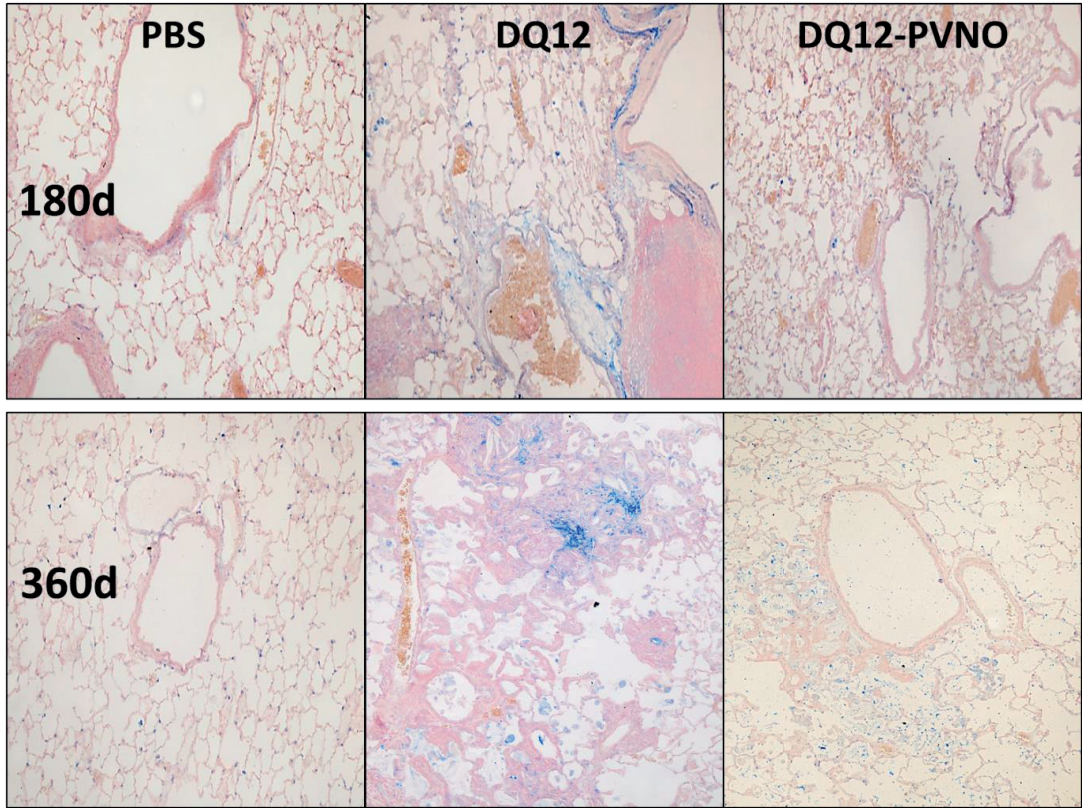


\title{
CHAPTER 8
}

\section{INFLAMMASOME ACTIVATION IN HUMAN SILICOSIS}

Paul M. Peeters ${ }^{1}$, Dirk Taeger ${ }^{2}$, Karin Sanders ${ }^{1}$, Gernot Rohde ${ }^{1}$, Georg Johnen ${ }^{2}$, Emiel F.M. Wouters ${ }^{1}$, Niki L. Reynaert ${ }^{1}$, Beate Pesch ${ }^{2}$.

${ }^{1}$ Department of Respiratory Medicine, Maastricht University Medical Centre+ (MUMC+), Maastricht University, Maastricht, The Netherlands

${ }^{2}$ Institute for Prevention and Occupational Medicine of the German Social Accident Insurance, Institute of the Ruhr-Universität Bochum (IPA), Buerkle-de-la-Camp-Platz 1, 44789, Bochum, Germany

\section{In preparation}

\begin{abstract}
Pneumoconiosis is a form of interstitial lung disease that is characterized by massive fibrosis and inflammation, which occurs after long-term inhalation of particulate matter. Silicosis is refractory to treatment and an important health problem. Inflammasome activation in vitro and in animal models of silicosis has been shown to be important in the disease process. Earlier, we demonstrated that silica is taken up by and initiates inflammasome activation in bronchial epithelial cells in vitro. Here we wanted to investigate if inflammasome activation occurs in epithelial lung tissue of miners who were diagnosed with silicosis versus nonsilicosis, how silica particles are taken up in bronchial epithelial in vitro and by which mechanism. Lung tissue was available of 40 miners who were diagnosed with silicosis by pathologists were matched to 40 miners, where there was no visible silicotic fibrosis. They were matched on the basis of birth ( $+/-5$ years), number of years ( $+/-5$ years) and the cumulative quartz exposure $(+/-5 \mathrm{mg} / \mathrm{m} 3 /$ years $)$. By immunohistochemistry, lung tissue slides were stained for Caspase- 1 and Interleukin-1 $\beta$ and scored semiquantitatively. Further statistical analysis revealed significantly elevated inflammasome activation in silicotic miners compared to non-silicotics in predefined compartments of the lung.
\end{abstract}





\section{INTRODUCTION}

Silicosis is a fibrotic pulmonary disease caused by the inhalation and accumulation of inorganic crystalline silicon dioxide or silica (SiO2). It is one of the oldest occupational diseases worldwide and the Occupational Safety and Health Administration (OSHA) estimates that 2.2 million people in the U.S. are still exposed to silica at the work place even today, including 1.85 million construction workers [1]. The prevalence of silicosis patients is in China, with 500,000 estimated cases of silicosis diagnosed between 1991 and 1995, with 6,000 new diagnosed incidents and over 24,000 deaths reported each year [2]. Although disease incidence has declined in developed countries, it is still prevalent and especially of health concern in many poorly surveyed countries with less compliance towards regulatory standards as well as protective and preventative measures of exposure to workers. The actual total figure might be much higher since under-diagnosis and under-reporting are quite common. No curative treatment exists currently against silicosis.

The impaction of crystalline silica particles on myeloid and epithelial cells in the lung has been described to lead to the assembly and activation of the multi-protein danger sensor, the inflammasome [3-5]. Its importance in the development of silicosis was demonstrated in mouse models as NLRP3 inflammasome deficient mice failed to develop inflammation and displayed attenuated fibrosis [6,7]. Inflammasome activation by silica thus seems to play an important role in the initiation and fueling of the inflammatory response, which is thought to underlie the process of fibrosis. Assembly of this well described multi-protein complex leads to caspase-1 enzymatic activation which in turn instigates the release of cytokines, alarmins and growth factors such as interleukin-1 $\beta$ (IL-1 $\beta$ ), IL-18, basic fibroblast growth factor (bFGF) and high-mobility group protein B1 (HMGB1) into the microenvironment $[8,9]$.

Despite this evidence for a critical role of the NLRP3 inflammasome and derived mediators in the development of silicosis in vitro and in mice, the presence and functional activation of this inflammasome has not been shown in clinical samples of silicosis. Therefore, the aim of this study is to investigate if the expression levels of inflammasome-related proteins, namely caspase- 1 and IL-1 $\beta$ are enhanced in lung tissue of miners that developed silicosis compared to miners matched for age and dust exposure without silicosis. 


\section{METHODS}

\section{Study population/ selection of cases}

From 1946 to 1990, about 400,000 miners have worked in the uranium mines of the former East German WISMUT Corporation. Since 1957, the Institute of Pathology in Stollberg, as part of the health service of the WISMUT Corporation, conducted autopsies of deceased miners with a focus on occupational lung diseases such as silicosis. After the German reunification this autopsy repository was opened to research. For each patient with a working history at the Sowjetisch-Deutsche Aktiengesellschaft/Sowjetische Aktiengesellschaft (SDAG/SAG) WISMUT, pathologists reviewed the corresponding tissue slides for assessment of silicosis in the lung $[10,11]$. Silicosis of the lung was defined as presence of fibrotic nodules with concentric "onion-skinned" arrangement of collagen fibers, central hyalinization, and a cellular peripheral zone, with lightly bi-refringent particles seen under polarized light in at least one of the tissue slides. For this study, which was authorized by the German government, 40 cases with silicosis and 40 cases without pathological silicosis were pair-matched for age and exposure data and further selected based on autopsy reports.

\section{Histopathology and Immunohistochemistry}

Tissue sections were stained with hematoxylin \& eosin (H\&E) and were reviewed by two pathologists at the Maastricht University Medical Centre (MUMC+, Maastricht, The Netherlands), to confirm the documented silicosis status based on the same criteria originally adopted. On H\&E stained slides the following alterations were evaluated according to severity and distribution: antracose-pigment sedimentation, broncho-alveolar hyperplasia, perivascular and peribronchiolar lymphocytic infiltration, alveolar histocytosis, alveolar lipoproteinosis, mixed cell alveolar inflammation and hyperplasia as well as perivascular fibrosis, fibrosis in alveolar septa, granulomas and silicotic nodules.

The lung tissue sections were deparaffinized and rehydrated. Heat-induced antigen retrieval using 1mM EDTA ( $\mathrm{pH} 8$ ) was performed with microwave technology (700 W or high for 10 min). After cooling down and immersing in cold TBS, the slides were incubated with 5\% bovine serum albumin (BSA, Sigma, St. Louis, MO) dissolved in Phosphate buffered saline (PBS) to block nonspecific antibody reaction. The following primary antibodies were diluted to final primary antibody concentrations of $2.5 \mu \mathrm{g} / \mathrm{mL}$ in $0.1 \%$ BSA (Sigma, St. Louis, MO) and incubated overnight at $4^{\circ} \mathrm{C}$ : mouse monoclonal caspase-1 (Santa Cruz Biotechnology sc56036) and IL-1 $\beta$ (Santa Cruz Biotechnology sc-7884). After washing with Tris-base buffered saline (TBS), tissue sections were incubated with rabbit anti-mouse or swine anti-rabbit (DAKO, Carpinteria, CA, USA) for 30 minutes at room temperature. To visualize proteins of interest, the biotin coupled $\mathrm{ABC}$ complex method was used with vector blue and counterstaining with nuclear fast red. Finally, tissue sections were treated with histosafe $(3 \mathrm{x} 3$ minutes) and mounted using vectamount. Control stainings were performed using an isotype control antibody, which revealed no staining.

\section{Semi-quantitative scoring}

Images taken at 25x, 100x, 200x and 640x using Zen Software were taken with the Axiophot Zeiss microscope. The slides were reviewed independently by two observers for assessment 
of immunohistochemical staining. Per section 4 fields were chosen and per field staining for six different compartments were evaluated: ${ }^{1)}$ alveolar epithelial cells, ${ }^{2)}$ bronchial epithelial cells, ${ }^{3)}$ endothelial cells, ${ }^{4)}$ macrophages, ${ }^{5)}$ proteinosis and ${ }^{6)}$ fibrosis. Next, the intensity of the staining was characterized using five predetermined scores $(0=$ none, $1=$ mild, $2=$ moderate, $3=$ above average, $4=$ high).

\section{Statistical methods}

To assess normality of distribution the Kolmogorov-Smirnov test was performed in SPSS. A p-value $>0.05$ indicated normal distribution and was represented with means and standard deviations whereas non-normal distributions were indicated with median and interquartile range as shown in Table 1. A Mann-Whitney $U$ test was performed with SAS (®) software to examine a significant difference between two groups. Univariate and bivariate analyses were used to examine correlations between the scores of staining of the proteins under investigation and quartz exposure data (Spearman's rho correlation coefficient, $\rho$ ). A p-value of $<0.05$ was considered significant. 


\section{RESULTS}

Evaluation of H\&E stained lung sections selected and matched for age, duration of quartz exposure (YOE, years of quartz exposure) and cumulative quartz exposure ( $\mathrm{mg} / \mathrm{m}^{3}$ * years) by 2 pathologists at the MUMC revealed that four cases in the group of silicotics and 2 cases in the non-silicotic group were misclassified according the original autopsy protocol. These cases were excluded from the study. Subject characteristics of the 36 miners with silicosis and 38 miners without silicosis of which the lung tissue was used in this study are summarized in Table 1. Both groups were well matched.

Table 1: Subject characteristics.

\begin{tabular}{|c|c|c|c|c|}
\hline \multirow{3}{*}{ Age at death (years) } & \multicolumn{2}{|c|}{ Silicotics $(\mathrm{n}=36)$} & \multicolumn{2}{|c|}{ Non-silicotics $(\mathrm{n}=38)$} \\
\hline & \multicolumn{2}{|c|}{$65(41-81)$} & \multicolumn{2}{|c|}{$69(48-82)$} \\
\hline & Mean & $\mathrm{SD}$ & Mean & $\mathrm{SD}$ \\
\hline \multirow[t]{2}{*}{ Cumulative quartz exposure $\left(\mathrm{mg} / \mathrm{m}^{3} \times\right.$ years $)$} & 14.5 & 9.7 & 14.9 & 8.4 \\
\hline & Median & Interquartile Range & Median & Interquartile Range \\
\hline Duration of quartz exposure (years) & 8.6 & 15.1 & 10.3 & 13.5 \\
\hline Duration working in Wismut mine (years) & 3.0 & 4.0 & 4.0 & 4.0 \\
\hline
\end{tabular}

In Figure 1, representative images of H\&E stained microscopic lung sections of one subject per group are shown. A typical example of pulmonary scarring in form of focal nodular lesions with particle deposition and inflammation, the concentric (onion skin) arrangement of collagen fibers with clear characteristics of hyalinization and peripheral cellularity, and massive fibrosis is shown for the silicotic group. In the non-silicotic group these characteristic silicotic nodules could not be observed, although other pathological effects of particle exposure such as mixed alveolar lipoproteinosis, neutrophylic granulocyte infiltration, mixed cell inflammation and fibrosis of alveolar septa are clearly detectable.
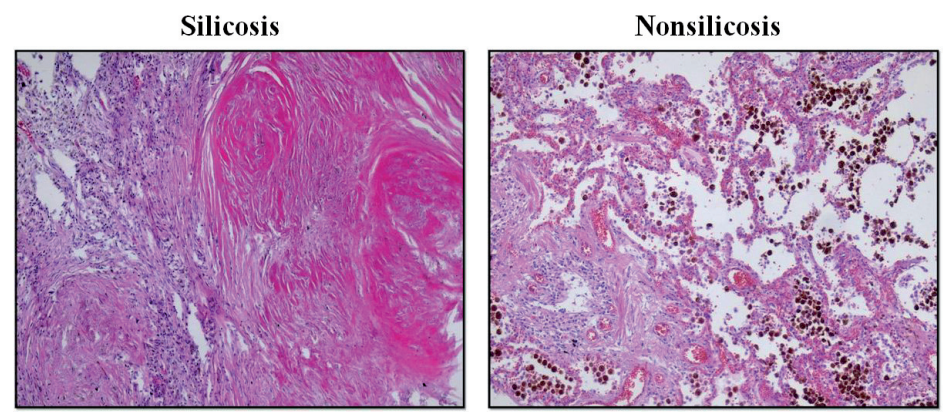

Figure 1: Histological features of samples from miners with or without silicosis Representative image of $H \& E$ stained lung tissue of one subject in the silicosis group, showing pulmonary scarring in form of nodular lesions with particles and inflammation+ and nonsilicosis group. with pathologically confirmed absence of silicotic nodules. 
We next investigated the presence of inflammasome-related proteins and possible differences in expression patterns between both groups. Caspase-1 was detected in alveolar cells $(\rightarrow)$, bronchial epithelium $(4)$, in regions with proteinosis exudates $(\mathrm{P})$, in fibrotic regions $(\mathrm{F})$ as well as in macrophages $(\Delta)$ and endothelial cells $(\varepsilon)$ (Figure 2). We semiquantitatively assessed the staining in each of these compartments individually and observed increased staining of caspase- 1 in each compartment in lung tissue of silicotic miners when compared to non-silicotics (Figure 3).

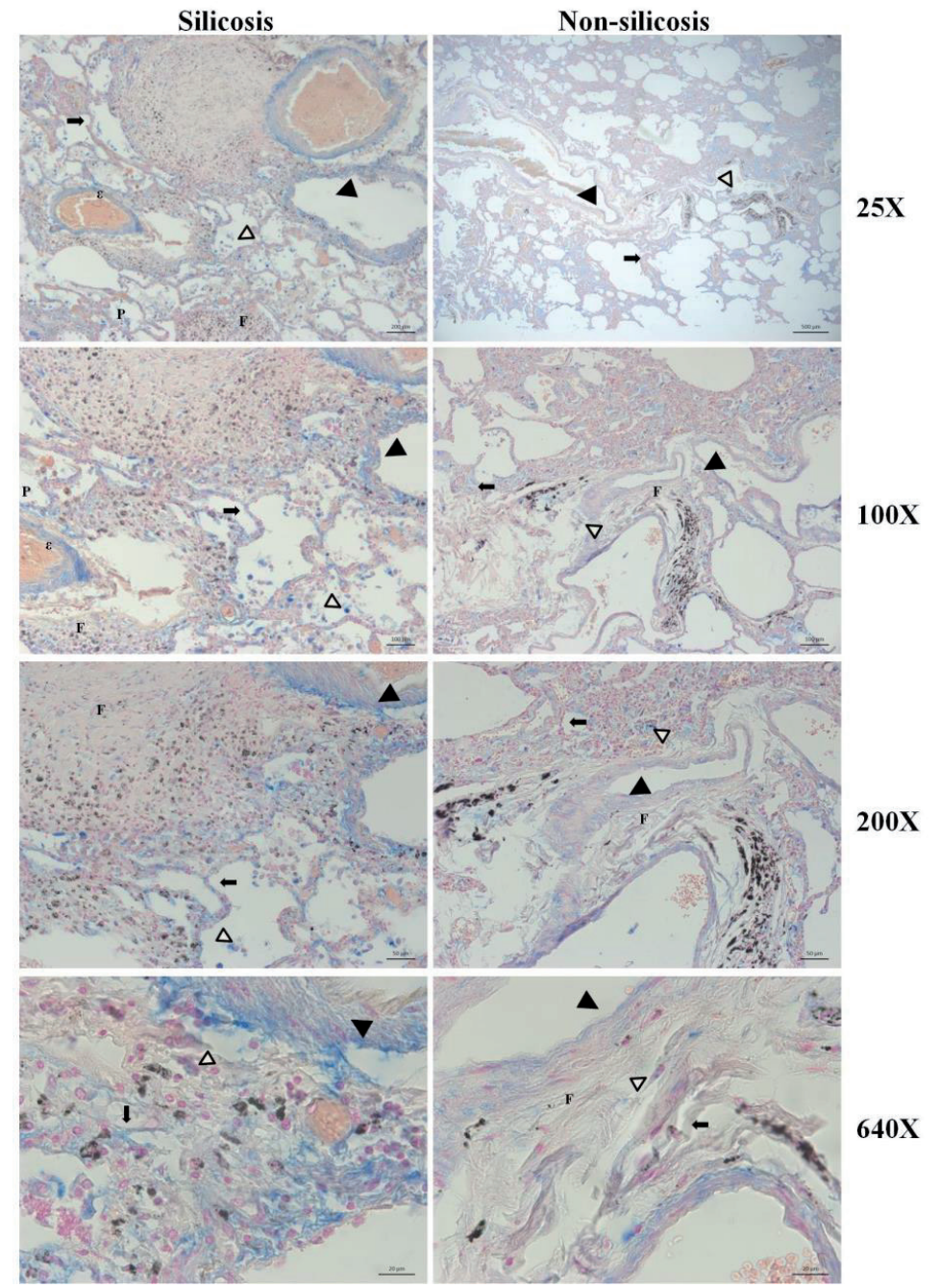

Figure 2: Representative images of caspase-1 immunohistochemistry. Caspase-1 expression was analyzed in human lung tissue by immunohistochemistry as a measure of inflammasome activation. Pro-form and active forms of the enzyme are detected by vector blue staining, tissues were counter stained using nuclear fast red. A representative image of one subject per group is shown. Symbols indicate caspase-1 expression in the alveolar compartment $(\rightarrow)$,

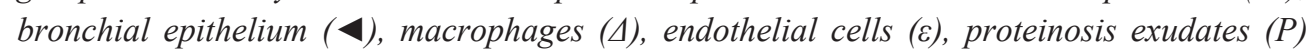
and fibrotic regions $(F)$. 
AL

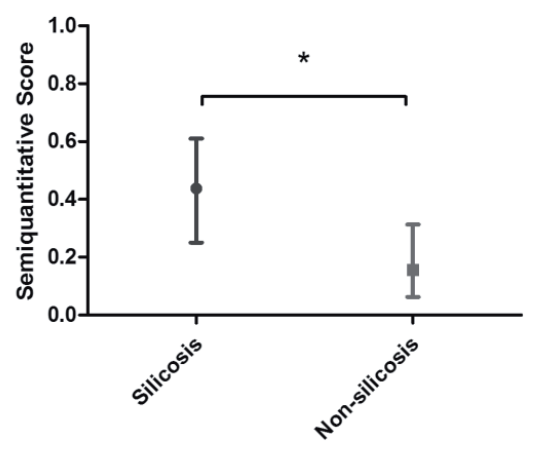

EN

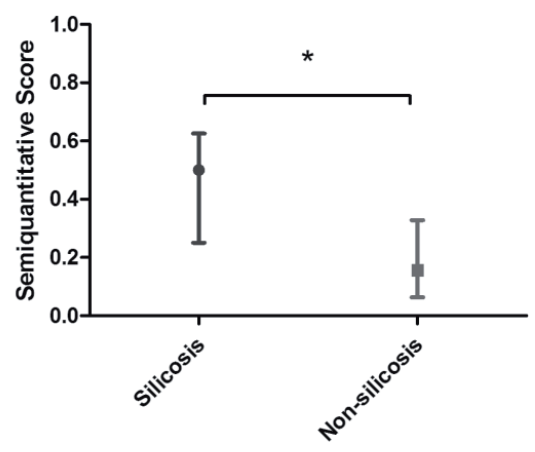

MA

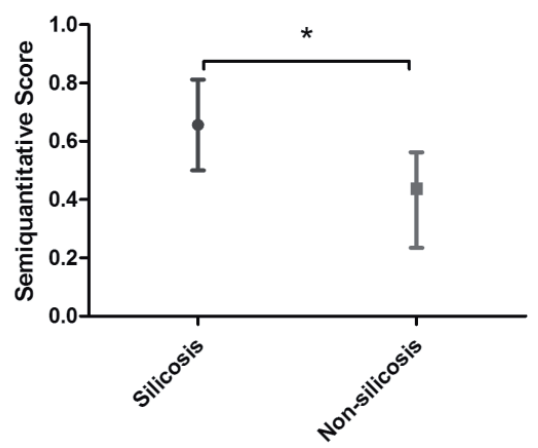

BR

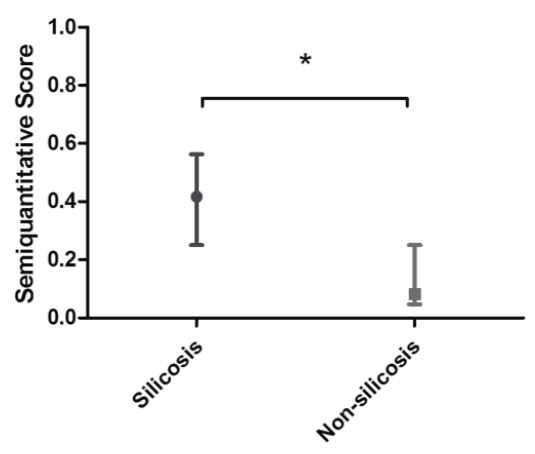

FI

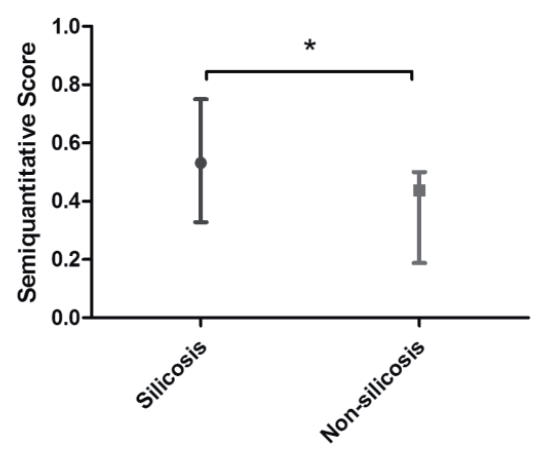

PR

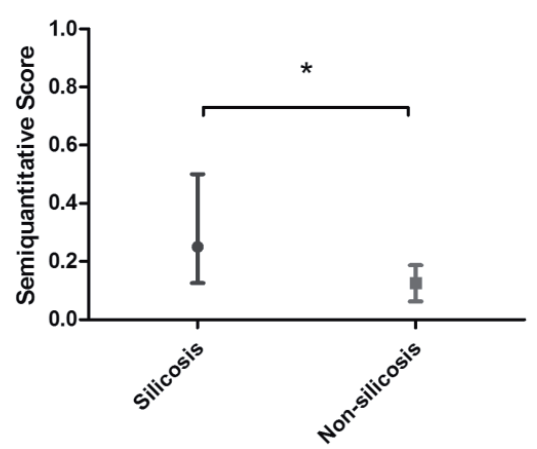

Figure 3: Semiquantitative caspase-1 expression levels in predefined compartments of lung tissue of miners who developed silicosis versus miners without silicosis. Compartments are indicated as AL (alveolar), BR (bronchial), EN (endothelial), FI (fibrotic), MA (macrophage) and PR (proteinosis). For all vertical line plots the mid-point indicates the median and the whiskers indicate the interquartile range. Significant differences are noted as * when $p<0.05$ 
IL-1 $\beta$ was detected also in alveolar cells $(\rightarrow)$, bronchial epithelium $(\varangle)$, in regions with proteinosis exudates $(\mathrm{P})$, in fibrotic regions $(\mathrm{F})$, as well as in macrophages $(\Delta)$ and endothelial cells $(\varepsilon)$ as shown in Figure 4. Based on semiquantitative analysis, statistically significant increased staining of caspase- 1 was observed in each compartment of silicotic miners when compared to non-silicotic subjects except in the endothelium and in exudate zones (Figure 5).

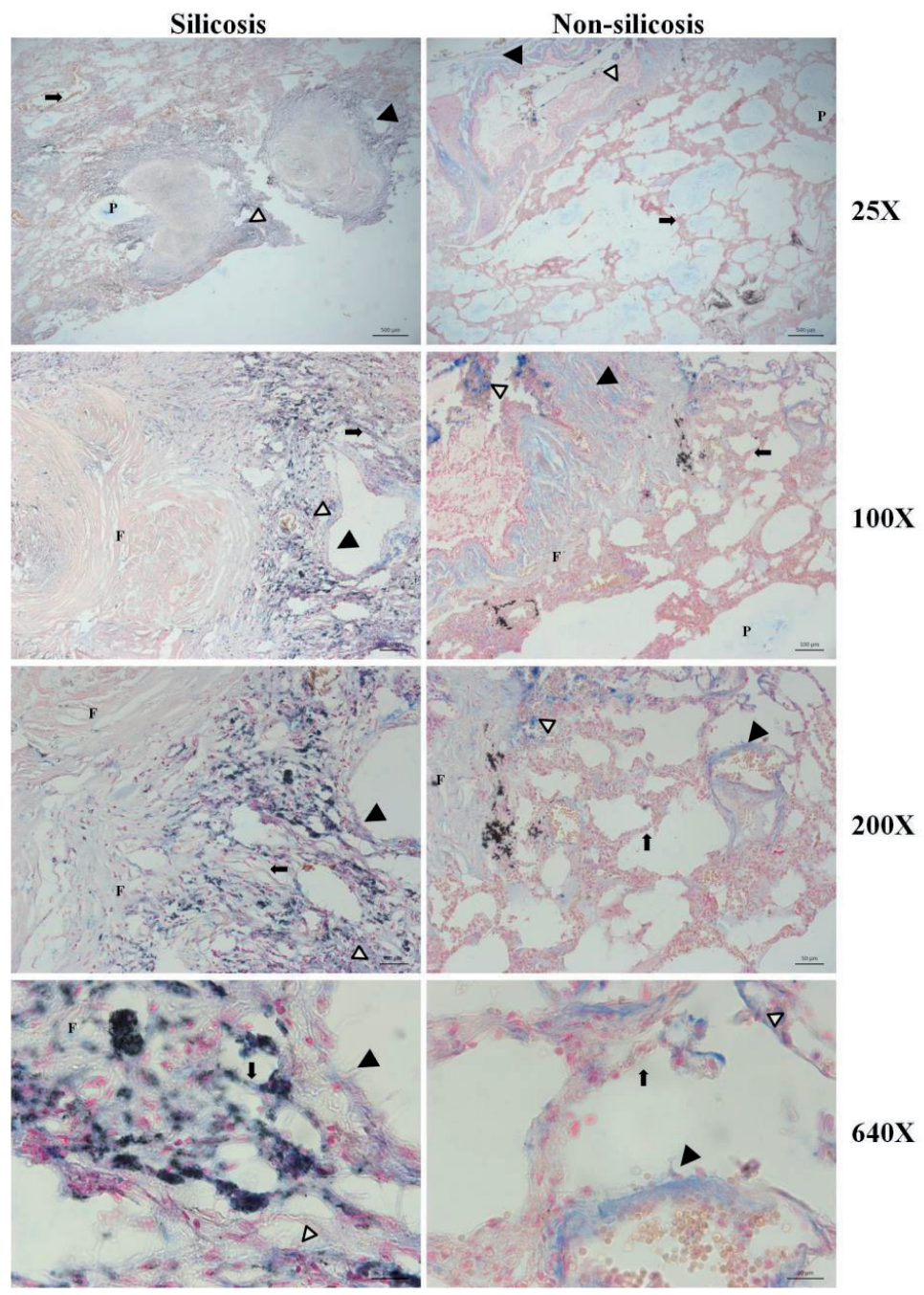

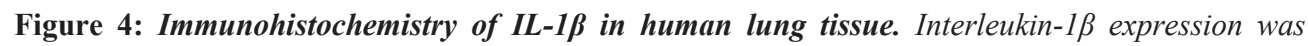
analyzed in human lung tissue by immunohistochemistry as a measure of inflammasome activation. Pro-form and active forms of the inflammatory cytokine are detected by vector blue staining, tissue was counter stained using nuclear fast red. A representative image of one subject per group is shown. Expression was detected in the alveolar compartment $(\rightarrow)$, bronchial epithelium $(\mathbf{4})$, macrophages $(4)$, proteinosis exudates $(P)$ and fibrotic regions $(F)$. 
AL

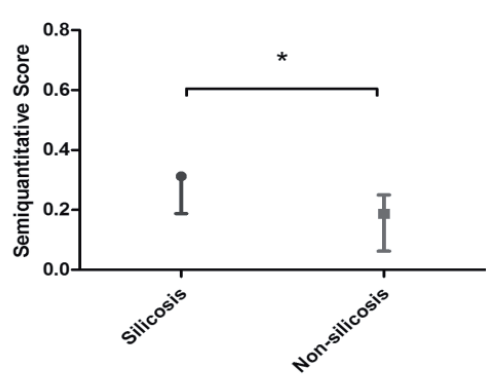

EN

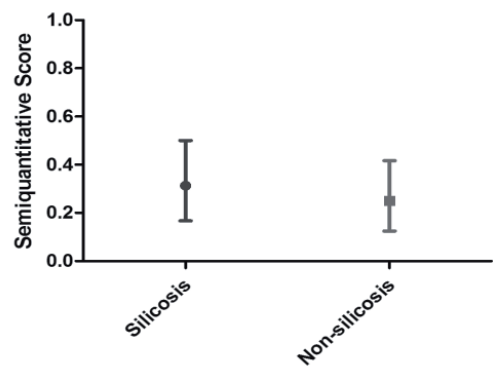

MA

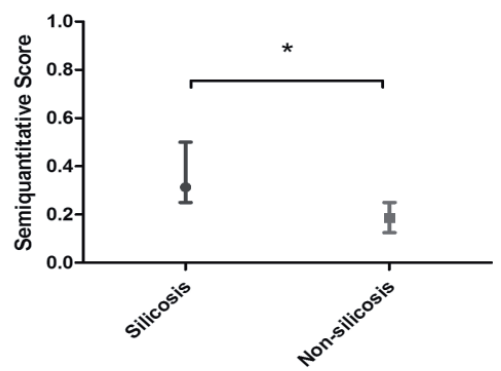

BR

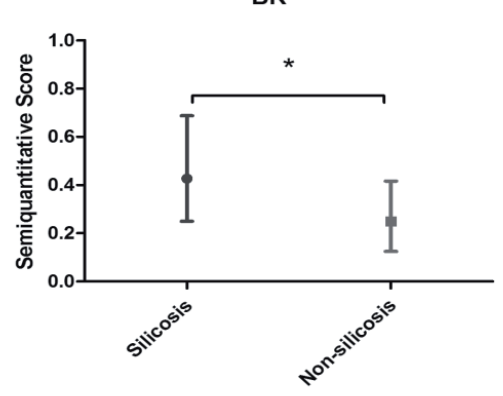

FI

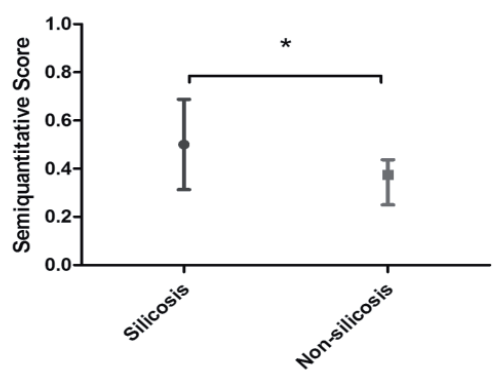

PR

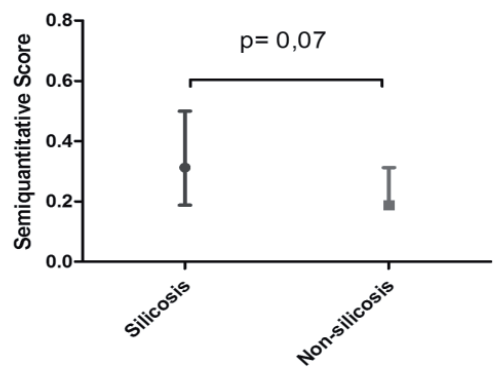

Figure 5: Semiquantitative interleukin-1 $\beta$ expression levels in predefined compartments of lung tissue of miners who developed silicosis versus miners without silicosis. Compartments are indicated as AL (alveolar), BR (bronchial), EN (endothelial), FI (fibrotic), MA (macrophage) and PR (proteinosis). For all vertical line plots the point indicates the median and the whiskers indicate the interquartile range. The asterix symbol above the connecting lines between plots represents significance $(p<0.05)$ after a pairwise comparison.

To further examine whether the amount and duration of quartz exposure was related to the expression levels of caspase-1 and IL-1 $\beta$, correlations between these parameters were examined. Within the group of silicotic miners, a statistically significant positive association was found between endothelial caspase- 1 expression and years of exposure $\left(R^{2}=329\right.$; $p$-value 0.038). IL-1 $\beta$ expression in the endothelium furthermore positively correlated with cumulative quartz exposure $\left(\mathrm{R}^{2}=0.327\right.$; $\mathrm{p}$-value 0.042$)$ in silicotics whereas no correlation was observed in non-silicotics. 


\section{DISCUSSION}

In this study, protein levels of caspase- 1 and IL-1 $\beta$ were found to be increased in lung tissue of miners that developed pathological silicosis compared to age and quartz exposure matched miners that did not develop silicosis. Since caspase-1 is the most appreciated read-out for inflammasome assembly and activation, and IL-1 $\beta$ the quintessential mediator released upon activation, these observations indicate significantly enhanced inflammasome activation in miners developing silicosis. Moreover, this activation was shown in a multitude of cellular compartments, including in macrophages, epithelial and endothelial cells.

To our knowledge, localization of inflammasome activation to different cellular compartments has not been investigated in humans before. Inflammasome activation by silica was initially characterized in macrophages in vitro or in macrophages isolated from dust exposed animals $[3,4,6,7]$. This study confirms that staining was observed in macrophages and that this was enhanced in the silicosis group. Statistically significant increased presence of both caspase- 1 and IL-1 $\beta$ in bronchial epithelial cells as well as in alveolar regions of silicotic miners was observed. In vitro, inflammasome activation in bronchial epithelial cells in response to silica treatment has been shown [5]. Furthermore, in an animal model of environmental exposure inflammasome activation in these cells was demonstrated in vivo as well [12].

The lung vascular endothelium is a critical component of the lungs that contributes to the pathogenesis of acute lung injury $[13,14]$. Endothelial cells are postulated to be amplifiers of inflammation through the sensing and release of IL-1 $[15,16]$. Post earthquake investigations in Japan revealed a higher silica concentration in BALF from diffuse alveolar hemorrhage $(\mathrm{DAH})$ patients indicating a link between DAH and the inhalation of earth quake dust [17]. Hemorrhagic shock has been shown to lead to the association of TRX/TXNIP with NLRP3, leading to inflammasome activation and IL-1 $\beta$ secretion [18]. Interestingly, our recent data revealed that TRX could prevent silica-induced inflammasome activation in bronchial epithelial cells (In Press) demonstrating its function in multiple cell types. Together, these data indicate that not only silica in endothelial cells but also hemorrhage itself, which is more general in silicotics than would be expected, activates the inflammasome and therefore could explain the link between mine dust exposure and features of hemorrhagic conditions that involve increased caspase-1 activity in lung endothelial cells.

Interestingly, inflammasome upregulation was shown in silicotics in particular. However, we did not find much staining in the hyalinized nodules and therefore, since the data are based on intensity scoring of caspase-1 and IL-1 $\beta$ in overall fibrous tissue, our significant differences could even be underestimated. Whether silicotic fibroblasts require the activation of the NLRP3 inflammasome only or other inflammasome subtypes are involved remains to be investigated. In line with the involvement of inflammasome in silicosis, it was already demonstrated that NLRP3 knockout mice develop less fibrosis in after exposure to silica [6]. This protection could be explained by the reduced levels of inflammation, but a direct effect of limiting inflammasome activation in fibroblasts could also underlie the phenotype. In support of the latter possibility, a study in systemic sclerosis (SSc), a disease characterized by fibrosis of the skin and visceral organs revealed increased expression of 40 genes associated with the inflammasome or downstream signaling molecules in fibroblasts derived from 
patients versus controls. Inhibition of caspase-1 in primary skin and lung fibroblasts abrogated the secretion of collagens, IL-1 $\beta$, and IL-18 [19]. Others reported caspase-1 expression in bronchiolar and alveolar epithelial cells, lymphocytes, neutrophils and macrophages in this bleomycin model [20]. These data suggest a key role for inflammasome activation in systemic fibrotic diseases.

This study has demonstrated that an enhanced inflammasome response is associated with the development of silicotic nodules and massive fibrosis in human silicosis. Inflammasome activation thus occurs in many cellular compartments and it is likely that it participates in disease pathogenesis through various autocrine and paracrine signaling loops. Indeed, nodule formation, the deposition of ECM in and destruction of parenchyma are the result of synergistic effects derived from multiple cellular compartments. The underlying processes are furthermore not discontinued because of positive feedback loops of which the persistence of inflammasome activation is an example.

Environmental materials are the major contributory factors to disease pathogenesis but not all individuals exposed to similar levels of exposure develop similar disease patterns which has led to the hypothesis of a genetic predisposition [21]. A substantial number of functional polymorphisms in inflammasome-related genes have been shown recently to influence individual susceptibility and are associated with the risk of development of pneumoconiosis. For example, polymorphisms in the gene coding for the interleukin-1 receptor antagonist, IL1RA, were associated with the risk of silicosis in Chinese workers exposed to silica particles [22]. In heat-shock proteins (HSPs), described as possible cofactors in inflammasome activation [23], a relationship between the genetic variation in constitutive and inducible HSP70 genes and the risk for coal workers' pneumoconiosis (CWP) among miners in southwestern China was also demonstrated [24]. Additionally, presence of genetic polymorphisms in NLRP3 itself conferred an increased risk for the development of CWP [25]. The differential inflammasome expression reported in this study supports a possible host predisposition rather than differences in expression levels fitting with the paradigm of a differential susceptibility to develop silicosis in humans. Moreover, given the positive correlations observed between indexes of exposure and inflammasome activation within the group of silicotic miners, this activation seems dose-related.

The tissue samples of the German autopsy repository were very well conserved over time and the paraffin lung tissue samples could still be evaluated for typical hallmarks of silicosis by means of H\&E staining, as described earlier [11]. In order to correctly classify the biopsies in silicotic and non-silicotic individuals, all slides were reviewed independently by 2 pathologists. Findings in 6 miners were discordant from the original classification and were excluded from the study.

Understanding the pathogenic processes promoting ECM deposition and hyalinization of collapsing bronchi and vessels in silicosis is of great importance to develop future therapeutic interventions in these and other fibrotic occupational disorders. Besides inflammasome activation in miners developing silicosis, this study demonstrated the association of inflammasome activation to exposure data in this group as well as involvement of different cellular compartments in disease development. 


\section{REFERENCES}

1. Steenland K, Ward E: Silica: a lung carcinogen. CA Cancer J Clin 2014, 64:63-69.

2. Leung CC, Yu IT, Chen W: Silicosis. Lancet 2012, 379:2008-2018.

3. Dostert C, Petrilli V, Van Bruggen R, Steele C, Mossman BT, Tschopp J: Innate immune activation through Nalp3 inflammasome sensing of asbestos and silica. Science 2008, 320:674-677.

4. Rada B, Park JJ, Sil P, Geiszt M, Leto TL: NLRP3 inflammasome activation and interleukin-1beta release in macrophages require calcium but are independent of calcium-activated NADPH oxidases. Inflamm Res 2014, 63:821-830.

5. Peeters PM, Perkins TN, Wouters EF, Mossman BT, Reynaert NL: Silica induces NLRP3 inflammasome activation in human lung epithelial cells. Part Fibre Toxicol 2013, 10:3.

6. Cassel SL, Eisenbarth SC, Iyer SS, Sadler JJ, Colegio OR, Tephly LA, Carter AB, Rothman PB, Flavell RA, Sutterwala FS: The Nalp3 inflammasome is essential for the development of silicosis. Proc Natl Acad Sci U S A 2008, 105:9035-9040.

7. Hornung V, Bauernfeind F, Halle A, Samstad EO, Kono H, Rock KL, Fitzgerald KA, Latz E: Silica crystals and aluminum salts activate the NALP3 inflammasome through phagosomal destabilization. Nat Immunol 2008, 9:847-856.

8. Martinon F, Burns K, Tschopp J: The inflammasome: a molecular platform triggering activation of inflammatory caspases and processing of proIL-beta. Mol Cell 2002, 10:417-426.

9. Keller M, Ruegg A, Werner S, Beer HD: Active caspase-1 is a regulator of unconventional protein secretion. Cell 2008, 132:818-831.

10.Taeger D, Bruning T, Pesch B, Muller KM, Wiethege T, Johnen G, Wesch H, Dahmann D, Hoffmann W: Association between lymph node silicosis and lung silicosis in 4,384 German uranium miners with lung cancer. Arch Environ Occup Health 2011, 66:34-42.

11.Wesch H EA, Mueller KM, Wiethege Th Radiologische Erfassung, Untersuchung und Bewertung bergbaulicher Altlasten - Gesundheitliche Bewertung. Schriftenreihe Reaktorsicherheit und Strahlenschutz Ergebnisberichte, Untersuchungen, Studien, Gutachten; 2005; 346 p 2005.

12.Hirota JA, Hirota SA, Warner SM, Stefanowicz D, Shaheen F, Beck PL, Macdonald JA, Hackett TL, Sin DD, Van Eeden S, Knight DA: The airway epithelium nucleotide-binding domain and leucine-rich repeat protein 3 inflammasome is activated by urban particulate matter. J Allergy Clin Immunol 2012, 129:1116-1125 e1116.

13. Matthay MA, Zimmerman GA, Esmon C, Bhattacharya J, Coller B, Doerschuk CM, Floros J, Gimbrone MA, Jr., Hoffman E, Hubmayr RD, et al: Future research directions in acute lung injury: summary of a National Heart, Lung, and Blood Institute working group. Am J Respir Crit Care Med 2003, 167:1027-1035.

14. Orfanos SE, Mavrommati I, Korovesi I, Roussos C: Pulmonary endothelium in acute lung injury: from basic science to the critically ill. Intensive Care Med 2004, 30:1702-1714.

15.Rao DA, Tracey KJ, Pober JS: IL-1alpha and IL-1beta are endogenous mediators linking cell injury to the adaptive alloimmune response. J Immunol 2007, 179:6536-6546. 
16. Shenkar R, Coulson WF, Abraham E: Hemorrhage and resuscitation induce alterations in cytokine expression and the development of acute lung injury. Am J Respir Cell Mol Biol 1994, 10:290-297.

17.Ebisawa K, Yamada N, Kobayashi M, Katahira M, Konno H, Okada S: Cluster of diffuse alveolar hemorrhage cases after the 2011 Tohoku Region Pacific Coast Earthquake. Respir Investig 2013, $51: 2-8$.

18.Xiang M, Shi X, Li Y, Xu J, Yin L, Xiao G, Scott MJ, Billiar TR, Wilson MA, Fan J: Hemorrhagic shock activation of NLRP3 inflammasome in lung endothelial cells. J Immunol 2011, 187:48094817.

19.Artlett CM, Sassi-Gaha S, Rieger JL, Boesteanu AC, Feghali-Bostwick CA, Katsikis PD: The inflammasome activating caspase 1 mediates fibrosis and myofibroblast differentiation in systemic sclerosis. Arthritis Rheum 2011, 63:3563-3574.

20.Kuwano K, Kunitake R, Maeyama T, Hagimoto N, Kawasaki M, Matsuba T, Yoshimi M, Inoshima I, Yoshida K, Hara N: Attenuation of bleomycin-induced pneumopathy in mice by a caspase inhibitor. Am J Physiol Lung Cell Mol Physiol 2001, 280:L316-325.

21.Katsnelson BA, Polzik EV, Privalova LI: Some aspects of the problem of individual predisposition to silicosis. Environ Health Perspect 1986, 68:175-185.

22. Wang YW, Lan JY, Yang LY, Wang De J, Kuang J: TNF-alpha and IL-1RA polymorphisms and silicosis susceptibility in Chinese workers exposed to silica particles: a case-control study. Biomed Environ Sci 2012, 25:517-525.

23. Mayor A, Martinon F, De Smedt T, Petrilli V, Tschopp J: A crucial function of SGT1 and HSP90 in inflammasome activity links mammalian and plant innate immune responses. Nat Immunol 2007, 8:497-503.

24.Zhang H, Jin T, Zhang G, Chen L, Zou W, Li QQ: Polymorphisms in heat-shock protein 70 genes are associated with coal workers' pneumoconiosis in southwestern China. In Vivo 2011, 25:251257.

25.Ji X, Hou Z, Wang T, Jin K, Fan J, Luo C, Chen M, Han R, Ni C: Polymorphisms in inflammasome genes and risk of coal workers' pneumoconiosis in a Chinese population. PLoS One 2012, 7:e47949. 


\section{CHAPTER 9}

DISCUSSION AND FUTURE DIRECTIONS 



\section{DISCUSSION}

\section{Distinct gene expression patterns by asbestos and silica in epithelial cells related to differential phenotypic outcomes}

The pathological presentation of asbestosis vs. silicosis is complex and unique. The severity and extent of lesions may reflect durability of particulates, host susceptibility factors such as impaired clearance by smoking or infection, and immune responses governing lung repair as elaborated on in Chapter 2. The intensity and duration of exposure as well as sites of accumulation of particulates also appear to play roles in governing susceptibility, development, and progression of these lung diseases [1,2]. Alternatively, in this thesis it was hypothesized that the differential pathological presentation of asbestosis vs. silicosis may be related to initial responses of lung epithelial cells and could be dissected using modern techniques of molecular and protein biology. Results in Chapter 3 and 4 expand the notion that gene expression studies can predict and link acute responses of respiratory epithelial cells, the first cell type to encounter asbestos fibers or silica particles after inhalation, to biofunctional pathways leading to lung disease. Overall, the data demonstrate for the first time early alterations in both common gene expression that can be linked to inflammation and fibrosis by both pathogenic minerals and unique changes by asbestos or silica that may be intrinsically linked to either the development of lung cancers and mesothelioma or to massive inflammation and progressive nodular fibrosis, respectively. Importantly it was shown that one challenge of mineral exposure to epithelial cells led to alterations in genes that have been demonstrated earlier in clinical samples and could be proposed as biomarker candidates. Moreover studies in Chapter $\mathbf{3}$ and $\mathbf{4}$ also reveal altered expression of novel molecules in response to cristobalite silica and crocidolite asbestos that can be pursued in functional studies, thus permitting their evaluation as new targets in prevention and therapy of fibroproliferative diseases and cancers. These novel data are in line with recent findings suggesting that the hazards of fibered dust mainly include the induction of direct toxicity by altering signaling pathways involved in carcinogenesis and proliferation, while granular dust shows indirect toxicity by altering signaling pathways involved in inflammatory processes [3]. Additionally, this work indicates that toxicological testing of particulates by surveying viability and/or metabolic activity is insufficient to predict their pathogenicity. Moreover, they show that acute responses of the lung epithelium can be indicative of pathologic potential using either immortalized lines or primary cells. Classical toxicity tests are still mandatory for many new and old 'controversial' particulates. Therefore assessment of the degree and magnitude of these responses by microarrays in vitro could be predictive in determining the pathogenicity of potentially harmful molecules. Crystalline silica was stated to be a Class I carcinogen by the IARC in 1997, despite inconsistent epidemiological data and the fact that its carcinogenic potential in non-smokers remains controversial. Our studies in Chapter 3 and 4 , lend further support to the classification by IARC, as carcinogenic pathways were shown to be induced by cristobalite silica in lung epithelial cells. Lastly, it should be noted that lung cancer induction by silica was demonstrated earlier in rats, but not in mice and hamsters, indicating species-dependent differences in epithelial and granulomatous reactions. 


\section{Inflammasome in epithelial cells}

A group of genes related to the inflammasome as well as to caspase-1 signalling and the proinflammatory cytokine IL-1 $\beta$ was identified in the available microarray dataset in the BEAS2B cell line and primary human bronchial epithelial (NHBE) cells exposed to silica and asbestos described in Chapter $\mathbf{3}$ and $\mathbf{4}$. The further aim of this thesis was to deliver a better understanding of inflammatory responses and mucosal immunity initiated by first line barrier epithelium in the lung in response to harmful particulates and fibers. Because of the emerging role of the inflammasome in a cadre of diseases, this danger sensing multiprotein complex became the main focus of investigation. As demonstrated by a first general review of inflammasome expression in epithelial cells in Chapter 5, these cells in many organs should be considered as highly important mediators of innate immune signaling. Further laboratory research to dissect out the role of epithelial inflammasomes to the mechanisms of inflammation in various organs is encouraged. Epithelial-specific conditional knockout models and transgenic animal studies will be a necessary approach to determine the importance of this contribution more profoundly. Next in Chapter 6, the presence and functional activation of the NLRP3 inflammasome by cristobalite silica in human lung epithelial cells was revealed. Cross-talk between epithelial cells and fibroblasts is a pivotal event contributing to the development of lung fibrosis. Basic fibroblast growth factor (bFGF) promotes the proliferation of fibroblasts and is indicated in the development of IPF. Our findings showed that inflammasome activation by silica in epithelial cells leads to enhanced bFGF release and is linked to fibroblast proliferation [4]. Interestingly, since our data were published others also demonstrated that conditioned medium from multiwall carbon nanotubes-treated bronchial epithelial cells induced inflammasome activation and significantly increased mRNA expression of pro-fibrotic markers (TIMP-1, tenascin-C, procollagen 1, and osteopontin) human lung fibroblasts. Furthermore, this study demonstrated reduced induction of pro-fibrotic markers when IL-1 $\beta$, IL-18 and IL-8 neutralizing antibodies were added to the conditioned medium or when conditioned medium from NLRP3 siRNA transfected cells was used [5]. These data thus paralleled and expanded upon our findings of epithelial cell directed and NLRP3-dependent fibroblast proliferation. These findings open new avenues in the development of more effective therapies for the numerous debilitating diseases with an acute and chronic inflammatory as well as fibrotic component.

\section{Role of crystallinity and polymorphs in inflammasome activation in vitro and in vivo}

During crushing and grinding of silica-containing materials, the reactive sianols $(\mathrm{SiOH})$ and dangling bonds $(\mathrm{SiO} \cdot)$ generated at the surface in cleavage planes may interact with molecular oxygen to produce surface radicals as well as particle-derived ROS such as hydrogen peroxide $(\mathrm{H} 2 \mathrm{O} 2)$ and damaging hydroxyl radicals $(\mathrm{HO} \bullet)$ as well as RNS. Inhaled particles are factors that disturb homeostasis and induce an inflammatory response. It has been established that freshly fractured silica dust has an increased pathogenic potential compared to aged silica dust because surface reactivity diminishes over time as reactive surface molecules dissipate [6]. These data suggest that oxidants are intrinsic to the damaging effects of these pathogenic particles after inhalation. Amorphous silica, as opposed to respirable crystalline silica particles, is less associated with lung inflammation and pulmonary fibrosis (silicosis) in experimental animal models and man. In order to further link the extent of changes in gene 
expression and cytokine production to silica crystallinity and pathogenicity in silicosis, amorphous particles and/or glass beads were used as controls in the experiments described in Chapters 4 and 6. Here it was demonstrated that gene expression changes as well as release of cytokines and chemokines from epithelial cells after asbestos or silica exposure, are less striking or absent after exposures to amorphous silica. The particle surface reactivity of crystalline silica has been long recognized as an important feature to their pathogenic hazard as reviewed by several authors [7, 8]. Effects of silica quartz on human lung epithelial cells and rat lung epithelial cells and macrophages have been shown to be determined by its surface reactivity [9-11]. Exemplary, surface modification using various compounds including aluminium or the polymer polyvinylpyridine $\mathrm{N}$-oxide (PVNO) drastically affected the ability of modified particles to generate ROS, to induced oxidative stress and downstream activation of pro-inflammatory signaling, as well as the development of pulmonary inflammation, toxicity and fibrosis in vitro and in vivo [12, 13]. In line with these findings, results in Chapter 7 indicate the pivotal role of surface reactivity of crystalline silica to activate the inflammasome in cultures of both epithelial cells and macrophages in vitro. Crystalline silica polymorphs, cristobalite silica and $\alpha$-quartz, increased inflammasome readout levels at equal surface-area characteristics and were demonstrated to be associated with caspase- 1 enzymatic activity in a surface reactivity dependent manner. In addition, DQ12 quartz exposure in rats induced acute and chronic functional activation of the inflammasome in the heterogeneous cell populations of the lung in association with its crystalline surface reactivity. In order to start addressing the etiological link between cell-particle surface interactions and subsequent inflammasome activation, it would first need to be established whether uptake in the cell is pivotal for subsequent reactions and imbalances of signaling that could lead to inflammasome activation. In Chapter 4 this uptake was demonstrated from 2 hours post exposure and in Chapter 6 uptake dependency of inflammasome activation was revealed. With respect to silica crystal induced carcinogenicity in rats the influence of PVNO coating on crystalline particle-induced inflammasome activation in relation to silica induced carcinogenesis suspensions could be examined further.

\section{Upstream mechanism leading to inflammasome activation}

Both cristobalite and amorphous silica particles interacted with cells at $2 \mathrm{~h}$ and were internalized by $24 \mathrm{~h}$ (Chapter 4). Studies with PVNO-coated particles revealed similar but delayed internalization and protective effects with respect to toxicity, DNA damage, and inflammation and fibrosis development $[11,12]$. In Chapter 6, the necessity of silica uptake to the induction of inflammasome activation was assessed by blocking active filament rearrangements in the cells. These experiments further demonstrated that silica induced inflammasome activation in epithelial cells was able to induce secondary fibroblast proliferation. ROS generation is essential for cell signaling and several important physiological responses, whereas excessive accumulation of ROS can lead to cellular damage and death. Furthermore, ROS production has been shown to be an inducer of inflammasome activation in myeloid cells [14-16] and in in vivo animal models [17, 18]. Uptake of silica polymorphs, NADPH oxidase activity and ROS formation has also been linked to NLRP3 inflammasome activation in THP-1 macrophages and bone marrow-derived dendritic cells 
$[17,19]$. In addition, exposure to silica is known to result in ROS generation in bone marrowderived macrophages from both wild type and Nlrp $3^{-/-}$mice, suggesting that ROS generation is upstream of NLRP3 activation [20]. Although we did not measure ROS levels, we have indications of ROS involvement in silica-induced inflammasome activation. First, it was demonstrated by others that PVNO coating inhibits the intrinsic ROS properties of DQ12 quartz $[11,13]$. Subsequently, it was shown that such modification abrogates the induction of oxidative DNA damage responses in lung epithelial cells in vitro $[11,21]$ and in rat lungs in vivo [13]. PVNO coating has also specifically been shown to abrogate the formation of ROS by phagocytic cells and lead to lower ROS levels in lavage fluid of exposed rats $[12,13]$. In association with this, PVNO modification also had profound effects on the induction and persistence of pulmonary inflammation [22], as well as the cellular uptake of silica particles and their clearance from rat lung [9]. Together with our additional proof for in vivo surface reactivity-dependent inflammasome activation by means of caspase-1 activity in the BALF we conclude that PVNO coating is associated with reduced ROS formation and reduced inflammasome activation.

In myeloid cells, inflammasome-activating ROS is predicted to be generated by a NADPH oxidase which is known to be assembled and activated upon phagocytosis of microbes and, importantly, inflammasome activation was abrogated by a NADPH oxidase inhibitor [15, 17]. The ability of TRX to reduce a variety of protein disulfides, suggests that it also possibly catalyzes the formation of correct disulfides during protein folding. TXNIP has been identified as a binding partner of reduced thioredoxin. TXNIP directly interacts with the redox-active domain of TRX, and is thought to function as a negative regulator of the TRX reductase activity. In normoxic homeostasis TXNIP is bound to TRX and after oxidative stress, the TXNIP-dependent repression of TRX is released. The dissociation of TRX from its inhibitory protein TXNIP is associated with increased levels of TXNIP in the cytosol. Zhou et al. demonstrated that after dissociation of the TXNIP-TRX complex, the unbound TXNIP positively regulated the NLRP3 inflammasome induced by uric acid crystals [15]. Accordingly, the TRX/TXNIP axis has been described as a redox switch, the redoxisome with the rational that increasing TRX or decreasing TXNIP expression, can reduce the possibility for unbound TXNIP to activate the inflammasome. Additionally, TXNIP is demonstrated to be a vital signaling connection linking ER stress and inflammation [23] and modulation of the TRX/TXNIP axis is reported to be beneficial for preventing hyper inflammation [24]. Extracellular thioredoxin was demonstrated to be incorporated into cells with intracellular effects [25]. Our findings of increased TXNIP mRNA levels upon exposure to silica fuelled the idea that the TRX/TXNIP axis could have an important regulatory function in epithelial inflammasome activation. In Chapter 7 the inhibitory capacity of the antioxidant TRX to inflammasome activation by overexpression as well as by exogenous administration was indeed demonstrated. A recent publication with experiments using asbestos fibers and their interaction with mesothelial cells accordingly associated a role for TRX as they showed that TXNIP downregulation decreased inflammasome activation and TRX overexpression prevented pyroptosis, proving again that this axis is not restricted to myeloid or epithelial cells [26]. 
Other hypothesized mechanisms, however not the main focus here, have been described to contribute to inflammasome activation [27-29]. Different damage-associated molecular patterns, such as silica crystals, aluminium salts, uric acid, cholesterol crystals, double-walled carbon nanotubes (DWCNTs) etc., can induce lysosomal destabilization when phagocytized or internalized [30-32]. This lysosomal damage or leakage, associated with cathepsin B activity, is linked to inflammasome activation [30-33]. Others report endogenous danger signals such as silica and uric acid crystals that conjecture a requirement for potassium efflux through the ATP-gated channel P2X7. Consistently phagosomal acidification, inhibition of cathepsin B's activity, antagonism of $\mathrm{P} 2 \mathrm{X} 7$ or blockade of potassium efflux were shown to blunt the activity of the inflammasome [34-36].

\section{Inflammasome activation in human silicosis}

Pneumoconiosis takes several years to develop depending on the exposure level of silica [1, 37]. In Chapter 8 data describe inflammasome activation in lung autopsy material of miners by means of its most appreciated activity readout caspase- 1 and its released mediator IL- $1 \beta$. In the analysis differential inflammasome expression between silicotic and non-silicotic miners was demonstrated. In addition this study provides insight into the relation between the development of typical silicotic nodules and inflammasome activation. Animal knock out studies demonstrated inflammasome dependence of the development of fibrotic hallmarks in mice $[17,20,38]$ and some inflammasome activation has been described in other inhalational diseases such as COPD [39-42]. However, for pneumoconiosis no other reports have described inflammasome activation in multiple cellular compartments of human lung samples. Environmental materials are the major contributory factors to disease pathogenesis but not all individuals exposed to similar levels of exposure develop similar disease patterns, which has led to the hypothesis of a genetic predisposition [43, 44]. Several reports have linked various single nucleotide polymorphisms in different genetic components of inflammasome-forming NLRs to the risk of development of pneumoconiosis and autoimmunity. For example, polymorphisms in the gene coding for NLRP3, heat-shock proteins (possible cofactors in inflammasome activation [45] as well as the interleukin-1 receptor antagonist were associated with the risk for coal workers' pneumoconiosis (CWP) and silicosis workers exposed to silica particles [46-49].

\section{FUTURE DIRECTIONS}

The challenge that lies ahead in this field of research is to establish mechanisms underlying toxicological responses to inhaled particles leading to interstitial fibrosis. For instance, by further studies on the effect of crystalline silica surface modifications in which we will employ polyvinylpyridine N-oxide (PVNO) coated silica. In parallel, the relative response of myeloid versus epithelial cells from wild-type and knock-out mice following silica exposure, next to bone marrow transplantation experiments and tissue specific knockout models, should be addressed.

Both silicosis and asbestosis can be induced by intratracheal instillation, by nasal or oropharyngeal aspiration, or by inhalation of mineral particles. A well-characterized silicabased model of inflammation and fibrogenesis can facilitate to gain insight into the role of age 
and aging in the development of pulmonary fibrosis in general. Given the data obtained and literature on the inflammaging theory of ageing possibly underlying a multitude of age-related diseases, it can be of particular interest to examine the involvement of the NLRP3 inflammasome in the processes of aging as well as fibrosis. Since physiological alterations occur with age in the immune and pulmonary systems, and silica activates NLRP3 in both, the focus should be on aging and senescence of both systems as well.

\section{CONCLUDING REMARKS}

Common and repeated cycles of fiber/particle uptake, inflammation, and deposition within the lung interstitium may perpetuate the development of asbestosis and silicosis. However, the pathologic presentation of both diseases is distinct and may progress in the absence of further exposures to minerals. Disease incidence has declined in the mining industry of developed countries but is still prevalent and especially of health concern in many poorly surveyed countries with less compliance towards regulatory standards as well protective measures and prevention of exposure to workers. No curative treatment exists currently against silicosis of which new outbreaks still occur occasionally. There are no effective regimens for treatment or cure of these diseases although new agents may be indicated based upon novel observations such as inflammasome activation and gene profiling that may be linked to early disease. As the number of new substances used in industry proliferates, the challenge to identify pathogenic substances increases. Many known pathogenic substances are being substituted by new materials, but the possibility that these new substances may be pathogenic requires vigilance. 


\section{REFERENCES}

1. Mossman BT, Churg A: Mechanisms in the pathogenesis of asbestosis and silicosis. Am J Respir Crit Care Med 1998, 157:1666-1680.

2. Cullinan P, Reid P: Pneumoconiosis. Prim Care Respir J 2013, 22:249-252.

3. Helmig S, Dopp E, Wenzel S, Walter D, Schneider J: Induction of altered mRNA expression profiles caused by fibrous and granular dust. Mol Med Rep 2014, 9:217-228.

4. Peeters PM, Perkins TN, Wouters EF, Mossman BT, Reynaert NL: Silica induces NLRP3 inflammasome activation in human lung epithelial cells. Part Fibre Toxicol 2013, 10:3.

5. Hussain S, Sangtian S, Anderson SM, Snyder RJ, Marshburn JD, Rice AB, Bonner JC, Garantziotis S: Inflammasome activation in airway epithelial cells after multi-walled carbon nanotube exposure mediates a profibrotic response in lung fibroblasts. Part Fibre Toxicol 2014, 11:28.

6. Dalal NS, Shi XL, Vallyathan V: ESR spin trapping and cytotoxicity investigations of freshly fractured quartz: mechanism of acute silicosis. Free Radic Res Commun 1990, 9:259-266.

7. Fubini B: Surface reactivity in the pathogenic response to particulates. Environ Health Perspect 1997, 105 Suppl 5:1013-1020.

8. Mossman BT, Glenn RE: Bioreactivity of the crystalline silica polymorphs, quartz and cristobalite, and implications for occupational exposure limits (OELs). Critical reviews in toxicology 2013, 43:632-660.

9. Albrecht C, Hohr D, Haberzettl P, Becker A, Borm PJ, Schins RP: Surface-dependent quartz uptake by macrophages: potential role in pulmonary inflammation and lung clearance. Inhalation toxicology 2007, 19 Suppl 1:39-48.

10. Hohr D, Steinfartz Y, Schins RP, Knaapen AM, Martra G, Fubini B, Borm PJ: The surface area rather than the surface coating determines the acute inflammatory response after instillation of fine and ultrafine $\mathrm{TiO} 2$ in the rat. Int J Hyg Environ Health 2002, 205:239-244.

11. Schins RP, Duffin R, Hohr D, Knaapen AM, Shi T, Weishaupt C, Stone V, Donaldson K, Borm PJ: Surface modification of quartz inhibits toxicity, particle uptake, and oxidative DNA damage in human lung epithelial cells. Chem Res Toxicol 2002, 15:1166-1173.

12. Albrecht C, Knaapen AM, Becker A, Hohr D, Haberzettl P, van Schooten FJ, Borm PJ, Schins RP: The crucial role of particle surface reactivity in respirable quartz-induced reactive oxygen/nitrogen species formation and APE/Ref-1 induction in rat lung. Respir Res 2005, 6:129.

13. Knaapen AM, Albrecht C, Becker A, Hohr D, Winzer A, Haenen GR, Borm PJ, Schins RP: DNA damage in lung epithelial cells isolated from rats exposed to quartz: role of surface reactivity and neutrophilic inflammation. Carcinogenesis 2002, 23:1111-1120. 
14. Cruz CM, Rinna A, Forman HJ, Ventura AL, Persechini PM, Ojcius DM: ATP activates a reactive oxygen species-dependent oxidative stress response and secretion of proinflammatory cytokines in macrophages. J Biol Chem 2007, 282:2871-2879.

15. Zhou R, Tardivel A, Thorens B, Choi I, Tschopp J: Thioredoxin-interacting protein links oxidative stress to inflammasome activation. Nat Immunol 2010, 11:136-140.

16. Schmidt RL, Lenz LL: Distinct licensing of IL-18 and IL-1beta secretion in response to NLRP3 inflammasome activation. PLoS One 2012, 7:e45186.

17. Dostert C, Petrilli V, Van Bruggen R, Steele C, Mossman BT, Tschopp J: Innate immune activation through Nalp3 inflammasome sensing of asbestos and silica. Science 2008, 320:674-677.

18. Franchi L, Eigenbrod T, Munoz-Planillo R, Nunez G: The inflammasome: a caspase-1activation platform that regulates immune responses and disease pathogenesis. Nat Immunol 2009, 10:241-247.

19. Winter M, Beer HD, Hornung V, Kramer U, Schins RP, Forster I: Activation of the inflammasome by amorphous silica and $\mathrm{TiO} 2$ nanoparticles in murine dendritic cells. Nanotoxicology 2011, 5:326-340.

20. Cassel SL, Eisenbarth SC, Iyer SS, Sadler JJ, Colegio OR, Tephly LA, Carter AB, Rothman PB, Flavell RA, Sutterwala FS: The Nalp3 inflammasome is essential for the development of silicosis. Proc Natl Acad Sci U S A 2008, 105:9035-9040.

21. Schins RP, Knaapen AM, Cakmak GD, Shi T, Weishaupt C, Borm PJ: Oxidant-induced DNA damage by quartz in alveolar epithelial cells. Mutat Res 2002, 517:77-86.

22. Albrecht C, Schins RP, Hohr D, Becker A, Shi T, Knaapen AM, Borm PJ: Inflammatory time course after quartz instillation: role of tumor necrosis factor-alpha and particle surface. Am J Respir Cell Mol Biol 2004, 31:292-301.

23. Oslowski CM, Hara T, O'Sullivan-Murphy B, Kanekura K, Lu S, Hara M, Ishigaki S, Zhu LJ, Hayashi E, Hui ST, et al: Thioredoxin-interacting protein mediates ER stressinduced beta cell death through initiation of the inflammasome. Cell Metab 2012, 16:265273.

24. Yoshihara E, Masaki S, Matsuo Y, Chen Z, Tian H, Yodoi J: Thioredoxin/Txnip: redoxisome, as a redox switch for the pathogenesis of diseases. Front Immunol 2014, $4: 514$.

25. Matsuo Y, Yodoi J: Extracellular thioredoxin: a therapeutic tool to combat inflammation. Cytokine Growth Factor Rev 2013, 24:345-353.

26. Thompson JK, Westbom CM, MacPherson MB, Mossman BT, Heintz NH, Spiess P, Shukla A: Asbestos modulates thioredoxin-thioredoxin interacting protein interaction to regulate inflammasome activation. Part Fibre Toxicol 2014, 11:24. 
27. Mariathasan S, Monack DM: Inflammasome adaptors and sensors: intracellular regulators of infection and inflammation. Nat Rev Immunol 2007, 7:31-40.

28. Schroder K, Tschopp J: The inflammasomes. Cell 2010, 140:821-832.

29. Sutterwala FS, Ogura Y, Zamboni DS, Roy CR, Flavell RA: NALP3: a key player in caspase-1 activation. J Endotoxin Res 2006, 12:251-256.

30. Meunier E, Coste A, Olagnier D, Authier H, Lefevre L, Dardenne C, Bernad J, Beraud M, Flahaut E, Pipy B: Double-walled carbon nanotubes trigger IL-1beta release in human monocytes through Nlrp3 inflammasome activation. Nanomedicine 2012, 8:987-995.

31. Biswas R, Hamilton RF, Jr., Holian A: Role of lysosomes in silica-induced inflammasome activation and inflammation in absence of MARCO. J Immunol Res 2014, 2014:304180.

32. An LL, Mehta P, Xu L, Turman S, Reimer T, Naiman B, Connor J, Sanjuan M, Kolbeck R, Fung M: Complement C5a potentiates uric acid crystal-induced IL-1beta production. Eur J Immunol 2014.

33. Hornung V, Bauernfeind F, Halle A, Samstad EO, Kono H, Rock KL, Fitzgerald KA, Latz E: Silica crystals and aluminum salts activate the NALP3 inflammasome through phagosomal destabilization. Nat Immunol 2008, 9:847-856.

34. Perregaux D, Gabel CA: Interleukin-1 beta maturation and release in response to ATP and nigericin. Evidence that potassium depletion mediated by these agents is a necessary and common feature of their activity. J Biol Chem 1994, 269:15195-15203.

35. Petrilli V, Papin S, Dostert C, Mayor A, Martinon F, Tschopp J: Activation of the NALP3 inflammasome is triggered by low intracellular potassium concentration. Cell Death Differ 2007, 14:1583-1589.

36. Qu Y, Franchi L, Nunez G, Dubyak GR: Nonclassical IL-1 beta secretion stimulated by $\mathrm{P} 2 \mathrm{X} 7$ receptors is dependent on inflammasome activation and correlated with exosome release in murine macrophages. J Immunol 2007, 179:1913-1925.

37. Leung CC, Yu IT, Chen W: Silicosis. Lancet 2012, 379:2008-2018.

38. Guo J, Gu N, Chen J, Shi T, Zhou Y, Rong Y, Zhou T, Yang W, Cui X, Chen W: Neutralization of interleukin-1 beta attenuates silica-induced lung inflammation and fibrosis in C57BL/6 mice. Arch Toxicol 2013, 87:1963-1973.

39. Birrell MA, Eltom S: The role of the NLRP3 inflammasome in the pathogenesis of airway disease. Pharmacol Ther 2011, 130:364-370.

40. Wanderer AA: Interleukin-1beta targeted therapy in severe persistent asthma (SPA) and chronic obstructive pulmonary disease (COPD): proposed similarities between biphasic pathobiology of SPA/COPD and ischemia-reperfusion injury. Isr Med Assoc J 2008, $10: 837-842$. 
41. Brusselle GG, Provoost S, Bracke KR, Kuchmiy A, Lamkanfi M: Inflammasomes in respiratory disease: from bench to bedside. Chest 2014, 145:1121-1133.

42. Eltom S, Stevenson CS, Rastrick J, Dale N, Raemdonck K, Wong S, Catley MC, Belvisi MG, Birrell MA: P2X7 receptor and caspase 1 activation are central to airway inflammation observed after exposure to tobacco smoke. PLoS One 2011, 6:e24097.

43. Katsnelson BA, Polzik EV, Privalova LI: Some aspects of the problem of individual predisposition to silicosis. Environ Health Perspect 1986, 68:175-185.

44. Yucesoy B, Luster MI: Genetic susceptibility in pneumoconiosis. Toxicol Lett 2007, 168:249-254.

45. Mayor A, Martinon F, De Smedt T, Petrilli V, Tschopp J: A crucial function of SGT1 and HSP90 in inflammasome activity links mammalian and plant innate immune responses. Nat Immunol 2007, 8:497-503.

46. Ji X, Hou Z, Wang T, Jin K, Fan J, Luo C, Chen M, Han R, Ni C: Polymorphisms in inflammasome genes and risk of coal workers' pneumoconiosis in a Chinese population. PLoS One 2012, 7:e47949.

47. Zhang H, Jin T, Zhang G, Chen L, Zou W, Li QQ: Polymorphisms in heat-shock protein 70 genes are associated with coal workers' pneumoconiosis in southwestern China. In Vivo 2011, 25:251-257.

48. Wang YW, Lan JY, Yang LY, Wang De J, Kuang J: TNF-alpha and IL-1RA polymorphisms and silicosis susceptibility in Chinese workers exposed to silica particles: a case-control study. Biomed Environ Sci 2012, 25:517-525.

49. Zhou Y, Kang Y, Zhang Z, Liu J: IL-1RA +2018 polymorphism and the susceptivity to pneumoconiosis: a Meta-analysis. Int J Clin Exp Med 2014, 7:2204-2208. 


\section{CHAPTER 10}

SUMMARY/SAMENVATTING 



\section{SUMMARY}

The aim in the first part of this thesis was to provide more insight into the pathobiology of occupational lung diseases by investigating how exposure to different crystalline and amorphous mineral particles and fibers can give rise to several changes in gene expression patterns in bronchial epithelial cells.

Already in the 16th century it was described that exposure to mineral particles is associated with pulmonary disorders including fibrotic lung diseases. Pneumoconiosis refers to a group of non- neoplastic lung diseases that result from the inhalation of organic and non-organic particles which are unable to be removed by the natural defense mechanisms in the lung tissue. Asbestosis and silicosis are the two most studied diseases associated with the accidental or occupational inhalation of different silica polymorphs.

In Chapter $\mathbf{2}$ of this thesis, we provide a literature study that bundles both physicochemical aspects and molecular mechanisms and experimental pathology within environmental toxicology and more specifically in the context of asbestosis and silicosis. In Chapter 3, an example is given of how gene expression analysis in an in vitro model can provide a prediction for the acute response of lung epithelial cells, the first cells that come in contact with asbestos fibers or silica particles. In addition, these reactions could be linked to biofunctional signal transduction cascades that can lead to specific forms of these lung diseases. Accordingly, many of these changes can be linked to clinical biomarkers in patient samples exposed to asbestos fibers or silica particles. In summary, our data indicate early changes in both common gene expression, which can be coupled to both pathogenic fibrosis caused by minerals, as well as unique gene expression changes by silica that promote connective tissue formation, and by asbestos which are inherent in the development of lung cancer and mesothelioma. Our analysis identified new target molecules which could contribute to innovations in the diagnosis and therapy of fibroproliferative diseases and cancer. Furthermore, in Chapter 4, we demonstrate that surface characteristics of crystalline silica are decisive for the acute response of lung epithelial cells. As a result, this in vitro model is an alternative tool instead of expensive and time-consuming in vivo toxicological animal studies in order to study the potential adverse effects of environmental particles.

For the second part of this thesis we investigated the response to exposure to harmful particles and fibers in epithelial cells of the lungs, with a focus on the inflammatory responses and the mucosal immunity. Given our findings in in vitro studies and the emerging role of the dangerdetecting multi-protein, called the inflammasome, this molecule was the main focus of research in this section.

In Chapter 5 we demonstrated, based on a review about inflammasome expression, a present variable set of inflammasome subtypes in epithelial cells of different organs, possibly working cooperatively to strengthen the immune response and distinguish themselves with respect to the release of several mediators. As a result, it appears that inflammasome activation and subsequent release of "alarming" proteins is not limited to macrophages, indicating that epithelial cells can be considered as important cells of the immune system.

With regard to the lungs, the inflammasome hitherto was mainly described in cells of the myeloid compartment. The presence and functional activation of the NLRP3 inflammasome in lung epithelial cells was therefore examined in Chapter 6. It was proved that cristobalite 
silica in human lung epithelial cells in vitro increases the processing and secretion of inflammatory and alarmin proteins in bronchial epithelial cells. Further, we demonstrated that the release of these proteins, such as interleukin- $1 \beta$, is regulated by the NLRP3 inflammasome and that secretion of bFGF and HMGB1 is dependent on silica particle uptake. An important observation was that fibroblast proliferation is affected by exposure to bronchial epithelial cell conditioned medium, which indicates a complex crosstalk between the various cell types described above and a possible role for the inflammasome in the development of lung fibrosis.

Subsequently in Chapter 7, it was confirmed that inflammasome activation also occurs after exposure to crystalline polymorphs. If the surface reactivity of these particles was modified, the release of NLRP3-associated inflammatory and fibrotic mediators and alarm signals from both lung epithelial cells and differentiated macrophages was affected. Further, thioredoxin had the potential to modulate silica-induced caspase-1 activity in both cell types. In the last part of this chapter, inflammasome activation was measured by means of the detection of caspase-1 cleavage products in the lavage fluid of quartz exposed rats at different time points. This pointed towards persistent inflammasome activation in different cellular and interstitial compartments of the rat lung, which could be reduced by surface modification of the quartz particles.

Using immunohistochemical experiments with human autopsy samples, it was possible in Chapter $\mathbf{8}$ to integrate the results of basic fundamental research and extrapolating them into clinical research. Here, we demonstrated semiquantitatively increased caspase-1 and interleukin- $1 \beta$ expression levels in multiple predetermined cellular compartments of the lung tissue of miners that have silicosis versus miners without silicosis. Moreover, the fact that both groups were exposed to similar amounts of dust suggests that exposure is not the only root of inflammasome activation. In addition to a possible role for inflammasome activation in silicosis development of miners, this study demonstrated the involvement of different cellular compartments in the development of the disease.

In summary, the studies described in this manuscript reveal possible alternative ways to diagnose interstitial lung diseases. As we were also able to show a significant role for inflammasome activation in lung epithelial cells and consequently on fibroblasts, two major cell types involved in the underlying inflammation and fibrotic aspects of silicosis, this multiprotein could be a very interesting therapeutic target. Especially since the possible extrapolation of the data into human experiments, the clinical relevance of these findings is increased. Our review regarding inflammasome expression and functional activation in multiple epithelial barriers of different organs further underlines the importance of continued research which in turn can create possible routes for innovations in the therapeutic field of a variety of inflammatory diseases. Overall, this research may thus give rise to the possible development of new diagnostic and therapeutic agents to monitor and combat the effects of the dust exposure. 


\section{SAMENVATTING}

Sinds de $16^{\text {de }}$ eeuw werd beschreven dat blootstelling aan minerale deeltjes geassocieerd is met longstoornissen waaronder fibrotische longziekten. Pneumoconiosis refereert naar een groep niet-neoplastische longziekten die ontstaan door het inhaleren van organische en nietorganische deeltjes die niet verwijderd kunnen worden door het natuurlijke afweersystem in het longweefsel. Asbestose en silicose zijn de twee meest bestudeerde longaandoeningen veroorzaakt door het inhaleren van verschillende silica polymorfen. Dit werd In hoofdstuk 1 beschreven als algemene inleiding.

De doelstelling van dit proefschrift is meer inzicht krijgen in de pathogenese van silicosis and asbestosis. In het eerste deel van dit proefschrift hebben we bestudeerd hoe blootstelling aan verschillende kristallijne en amorfe minerale deeltjes alsook vezels aanleiding kan geven tot diverse veranderingen in genexpressie patronen in bronchiale epitheliale cellen, en hierdoor meer inzicht te krijgen in de pathobiologie van interstitiële longaandoeningen.

Hoofdstuk 2 omvat een literatuurstudie die zowel de fysico-chemische aspecten als de moleculaire mechanismen en de experimentele pathologie binnen de omgevings toxicologie beschrijft, met focus op asbestose en silicose. Tot nu toe bestaan er geen effectieve behandeling voor het genezen van beide ziektebeelden die een uiteenlopende histopathologisch beeld hebben. Omdat asbestose en silicose zo distincte histologische karakteristieken vertonen werd in hoofstuk 3, in een in vitro model, een voorbeeld gegeven hoe genexpressie analyse een predictie kan verschaffen wat betreft de acute reactie van long epitheel cellen, die als eerste in contact komen met asbest vezels of silica deeltjes. Bovendien konden deze reacties gelinkt worden aan biofunctionele signaaltransductie cascades die geassocieerd zijn met specifieke vormen van deze longziekten. Veel van deze veranderingen kunnen worden gekoppeld aan biomarkers in klinische patiënten materiaal blootgesteld aan asbestvezels of silica deeltjes. Kortom, onze data geven vroege veranderingen aan in zowel de gemeenschappelijke genexpressie, die kan worden gekoppeld aan fibrose veroorzaakt door beide pathogene mineralen, alsook aan unieke genexpressie veranderingen door silica die bindweefselvorming bevorderen en door asbest die inherent zijn aan de ontwikkeling van longkanker en mesothelioom. Onze analyses brachten ook vele nieuwe moleculen aan het licht die in functionele studies kunnen worden getest als mogelijk doelwit wat zou kunnen bijdragen aan innovatie in detectie en therapie van fibroproliferatieve ziekten en kanker. Verder werd in hoofdstuk 4 onderbouwd op basis van de vergelijking met amorfe silica dat de oppervlakte karakteristieken van kristallijn silica bepalend zijn voor de acute reactie van longepitheelcellen. Hierdoor is er met dit in vitro model een alternatieve methode voor handen ten opzichte van duurdere en tijdrovende in vivo toxicologische dierstudies om mogelijke predicties te doen wat betreft de potentiële schadelijke effecten van omgevingsdeeltjes.

Het tweede deel van dit proefschrift had het doel om een beter begrip te krijgen wat betreft ontstekingsreacties en mucosale immuun reacties die ontstaan als reactie op schadelijke deeltjes in long epitheel cellen. Gezien bevindingen in onze genexpressie studies en vanwege de opkomende rol van het gevaar detecterend multiproteine, het inflammasoom genaamd, 
werd dit complex in het tweede deel van het proefschrift de belangrijkste focus van het onderzoek.

In hoofdstuk 5 werd door middel van een overzicht van inflammasoom expressie in epitheliale cellen van verschillende organen een set aan inflammasoom subtypes benoemd, die mogelijk coöperatief de immuun respons kunnen versterken en zich onderscheiden in de vrijstelling van verschillende mediatoren. Hiermee tonen we aan dat inflammasoom activatie en daaropvolgende vrijzetting van "alarmerende" eiwitten niet beperkt is tot macrofagen en dat epitheelcellen beschouwd kunnen worden als zeer belangrijke cellen van het immuunsysteem.

Een belangrijke rol voor het inflammasoom in silicose werd eerder aangetoond, maar dit gaat voornamelijk uit van een rol in professionele immuuncellen. In hoofdstuk 6 was de aanwezigheid en functionele activering van het NLRP3 inflammasoom door cristobaliet silica in humane long epitheelcellen in vitro onderzocht. Er werd bewezen dat silica de verwerking en secretie vergroot van ontstekings- en alarm eitwitten in bronchiale epitheelcellen. Verdere aanwijzingen dat vrijzetting van deze eiwitten, zoals interleukin-1beta, wordt gereguleerd door het NLRP3 inflammasoom en dat de secretie van bFGF en HMGB1 afhankelijk is van de opname van de silica deeltjes. Een belangrijke observatie was ook dat fibroblast proliferatie beïnvloed wordt door blootstelling aan bronchiale epitheliale cellen geconditioneerd medium wat duidt op een belangrijke communicatie tussen de verschillende celtypen hierboven beschreven en een mogelijke rol voor het inflammasoom in de ontwikkeling van longfibrose.

Aansluitend hierop en met de kennis van hoofstuk 4 waarin we het belang van oppervlakte eigenschappen van silica deeltjes aantoonden, werd in hoofdstuk 7 bewezen dat deze inflammasoom activatie ook optreedt na blootstelling aan kristallijne polymorfen en dat de oppervlakte reactiviteit van deze deeltjes de vrijzetting van NLRP3-geassocieerde inflammatoire en fibrotische mediatoren en alarmsignalen van zowel longepitheelcellen als gedifferentieerde macrofagen kon beïnvloeden. Verder kon het antioxidant eiwit thioredoxine de silica-geïnduceerde caspase-1 activiteit in beide celtypes dempen. Bovendien werd in dit hoofdstuk inflammasoom activatie, aan de hand van de detectie van gekliefde caspase-1 producten in lavage vocht van silica blootgestelde ratten gestaafd op verschillende tijdstippen na blootstelling aan kwarts. Hierbij toonden we met immunohistochemie aanhoudende inflammasoom activering, die wordt verminderd door oppervlaktemodificatie van de kwartsdeeltjes, aan in verschillende cellulaire en interstitiële compartimenten van de rat long. Met behulp van immunohistochemische experimenten op humaan autopsie materiaal was het in hoofdstuk 8 mogelijk om de resultaten van het hoger beschreven fundamenteel onderzoek te extrapoleren naar de kliniek. Hier laten we verhoogde expressie niveaus zien van caspase-1 en interleukine-1 $\beta$ in meerdere cellulaire compartimenten van het longweefsel van mijnwerkers met silicose in vergelijking tot mijnwerkers zonder silicose. Daarenboven is de blootstelling gelijk tussen beide groepen en kan dus gezegd worden dat blootstelling alleen niet aan de basis ligt van inflammasoom activatie. Naast een mogelijke rol voor inflammasoom activatie in silicose ontwikkeling van mijnwerkers, toonde deze studie de betrokkenheid aan van verschillende cellulaire compartimenten in de ontwikkeling van de ziekte. 
Samengevat beschrijven de studies uitgevoerd in dit proefschrift een mogelijk alternatief voor de diagnose van interstitiele aandoeningen. Aangezien we in staat waren om een belangrijke rol toe te schrijven aan inflammasoom activatie in epitheelcellen en vervolgens een interessant effect zagen op fibroblasten, twee belangrijke celtypes betrokken bij de onderliggende ontsteking en fibrotische kenmerken van silicose, kan dit multiprotein een aanmerkelijk therapeutisch doelwit zijn. In het bijzonder door de extrapolatie van onze data in humane experimenten, is de klinische relevantie van deze bevindingen en dus de aandacht tot focus op dit therapeutisch doelwit molecule nog sterker toegenomen. Het belang van de inflammasoom in de epitheliale barrières van verschillende organen geeft aanleiding tot verder onderzoek en mogelijke routes voor innovaties in het therapeutische gebied van diverse ontstekingsziekten Kortom, dit onderzoek kan derhalve leiden tot de mogelijke ontwikkeling van nieuwe diagnostische en therapeutische middelen tegen de effecten van de stofblootstelling. 



\section{VALORISATION PARAGRAPH}





\section{VALORISATION PARAGRAPH}

By definition, pneumoconiosis refers to a group of diseases that are caused by the inhalation of organic and inorganic dusts that remain in the lung. It is one of the most important occupational lung diseases especially in under developed countries. Many fibrotic diseases nowadays are furthermore becoming leading causes of morbidity and mortality in the aging population of western society. Each year circa 450.000 persons die because of the consequences of pneumoconiosis. Although disease incidence has declined in developed countries, it is still prevalent and especially of health concern in many poorly surveyed countries with less compliance towards regulatory standards as well as protective and preventative measures of exposure to workers. The actual total figure of prevalence and incidence of death might be much higher since under-diagnosis and under-reporting are quite common. No curative treatment exists currently against silicosis, stressing the urgency of the problem. While significant progress has been made in our understanding of the biology of inflammation, fibrosis and aging in recent years, their interrelations remain largely speculative. Further investigation of the real etiopathology of the disease is therefore still very important. In this thesis, it is reported that crystalline particles and fibers activate an important multiprotein named the inflammasome (and possibly also other subtypes) which is involved in the progress of the disease symptoms and its development. The observation that it was possible to extrapolate in vitro and animal work data of visualized inflammasome activation into the clinical patients with pneumoconiosis has, in addition to its scientific evidence, a huge social relevance for many reasons.

In addition to the academic community, these research results can be important for a multitude of persons with a broad spectrum of varying interests. First of all, miners or others that are occupationally or accidentally exposed, but also their family members or civilians with much interest in medical facts or environmental research can benefit from the important new information. Dissemination of awareness about the irreversibility of the disease will lead to better preventive strategies for miners as well as mining companies to reduce the risk of exposure. Occupationally exposed workers will be more informed than before and everywhere it can be expected to have better compliance to reduce risk standardized protocols. This has a big impact on general safety and will lead to improved patient management. Unfortunately, a paradigm shift with respect to worldwide preventive approaches is still mandatory.

The results could immediately lead to adapting standard operating protocols by including immunological staining in biopsy material from persons with a high risk factor for disease development patients. Implementing additional methodology to enrich diagnostic medical imaging procedures to screen for disease pathology in humans will be innovative. As a result and for obvious reasons insurance companies that are occupied with single or familial autopsy cases will also benefit from the exposure to these research results. With no huge risks involved, new standard operating procedures for workers either or not related to occupational medicine can be implemented consisting of biomarker measurements in lavage fluid of 
patients or by staining resection material, all in parallel with existing medical imaging procedures.

With indications of new avenues in silicosis diagnostics supporting ongoing medical imaging it is clear that research and development departments of pharmaceutical companies will be very interested to start and evaluate the processing of new diagnostic kits that will facilitate early diagnosis of the disease. In the field of biopharmaceutical developments, early detection via the development of disease with diagnostic kits could improve quality of life of hundreds of thousands of workers annually worldwide. Moreover, there could be an impact economically as further substantiated results will lead to attract funding, spin-off collaborations and the creation of more jobs. With respect to pharmaceutical diagnostic tools, product research \& development and public relations as well as media we see largely enhanced market opportunities. With optimized communication tools and collaborators in taskforces the cost can remain very low. Development of innovative kits by spin-off or pharmaceutical companies will be very costly depending on size of production and types of specific antibodies, however the return of investment is gigantic and societal impact on job creation could also be improved. Aside from the existing product developing companies, it could also be interesting for startup entrepreneurs that have the idea to think globally on mask fabricating industry in line with offering optimized tools to people that may help decrease the risk of exposure to harmful particulates. Lastly, animals and animal activists profit since the findings will help to refine, reduce and replace the animal experiments in the future. All together, these results will have a not negligible societal impact. 


\section{ACKNOWLEDGEMENTS/ DANKWOORD}





\section{ACKNOWLEDGEMENTS/ DANKWOORD}

The past five years I had the fortune to work with wonderful people in the fascinating period of my PhD project. Now, I can finally thank everyone that helped me to fulfill this project and contributed to maximize my learning curve.

Beste Professor Wouters, u ligt aan de basis van het tot stand komen van deze thesis. Van in het begin hebt $\mathrm{u}$ me volledig vertrouwen en vrijheid gegeven in mijn experimenten. Jouw duidelijke visie en verwachtingen ondersteunden de gezonde druk om productief en efficient te zijn in het bereiken van onze output. De kans die $\mathrm{u}$, als mijn promotor, aangeboden hebt om initieel twee jaar te werken aan het departement Pathologie van de University of Vermont zal me altijd bijblijven. Deze levensles heeft ontzettend veel bagage opgeleverd om verder mijn weg te plaveien naar een interessante carriere. Hoewel niet altijd alles van een leien dakje liep, heb ik me ginds uitermate kunnen focussen op het onderzoek, netwerken en zelfontplooiing als individu en academicus. Mede omdat u steeds bereid was om ook vanop afstand het juiste wetenschappelijke en diplomatieke advies te geven. Ik ben enorm dankbaar dat $\mathrm{u}$ me steeds oog deed hebben om mijn onderzoekstopic topic mede vanuit een klinisch perspectief te benaderen. Hierdoor is dit proefschrift een mooi bench-to-bedside verhaal geworden. Bedankt voor uw input bij de verschillende internationale samenwerkingen en om bij te dragen aan het stand komen van mijn ERS fellowship. Ik kijk uit naar verdere projecten samen in de toekomst.

Beste Niki, je zult het wel met me eens zijn dat naast dit proefschrift er nog een boekje zou kunnen geschreven worden over de voorbije 5 jaar. Het was mijns inziens fantastisch! We hebben goed gelachen en tegelijkertijd kon ik ook steeds bij je terecht als het ging over bepaalde extra-professionele onderwerpen of issues. Ik dank je geduld met me! Ik ben er me van bewust dat je vaak zult gevloekt hebben bij verbeteringen van bepaalde stukken tekst. Uw gevatte commentaren en snelle evaluaties hebben ervoor gezorgd dat dit boekje is geworden tot wat het is en dat ik naadloos heb kunnen overschakelen in mijn huidige fellowship. Onvoorstelbaar knap vind ik het hoe je steeds vanuit jouw invalshoek meer klaarheid kan brengen in de complexe onderwerpen van mijn thesis en het werk van collegas uit de long-, spierlijn en andere departementen. Ik heb ontzettend veel geleerd van je wat betreft wetenschap maar ook wat betreft omgaan met mensen en je stempel drukken op de zaak. Ik zal altijd dankbaar zijn voor uw steun en ik wens u nog een prachtige verdere carriere en privéleven. Thanks, ik had me geen betere project begeleidster kunnen wensen.

Other people in Vermont have also been of great importance in the fulfilling of my work and achieving my goal. Off course I need to start with Professor Mossman, who introduced me in the field of particle and fiber toxicology. Thank you for your input in my work and supervising me as good as possible. Also thanks to everybody from the Shukla Lab for the help with finding my way everywhere in and outside the university and with experimental design of the studies. I have much appreciation for everyone from the group of Yvonne Janssen - Heininger as well. I remember a lot of our talks and meetings with your team, Yvonne. Thank you for having the doors always open and supporting in difficult moments. 
Prema and Jos, we will definitely meet further and I wish you and your family all the best together.

Ik wil IEDEREEN van de LONG-, SPIERLIJN en het secretariaat van het departement pulmonologie oprecht bedanken om altijd positief en behulpzaam te zijn indien ik iets vergeten was of advies wilde. Bedankt Mieke, Juanita, Harry, Ramon dat jullie deur altijd open stond voor advies of een interessante babbel. Thanks Koen, Alex en Nicky for the good party vibes, veel succes! Tim, the fun and serious moments back in Vermont and the last years in Maastricht have been very interesting. Thank you for your collaboration in our scientific output. Congrats with your work and lots of success in the future. Alle andere $\mathrm{PhD}$ collegas: Poornima, Ilse, Ana, Karin, Rosanne, Judith, Coby, Jules, Anita, Pieter... i wish you all the best. Natuurlijk wil ik alle technicians die een heleboel vragen kregen wanneer experimenten geoptimaliseerd dienden te worden ook de verf zetten; Renske, Claudia, Chiel, Marco, Frank en Anon bedankt voor jullie hulp, geduld, inzichten en voor de fijne momenten. De etentjes, borrels en labuitjes hebben altijd deugd gedaan. Wat een fijne groep zijn jullie en hopelijk zien we elkaar nog genoeg terug in de toekomst. Tot in mijn kleinste long- en spiervezeltjes, bedankt en veel succes aan jullie allen!

Roel and Catrin and all the colleagues at the IUF: thanks for the warm welcome in Düsseldorf and the ongoing joint efforts. The opportunity to work at your institute in the interesting field of environmental medicine is amazing.

Roommate Woebie, twas altijd leuk om gedachten te wisselen wat betreft ons onderzoek en privéleven. Knap hoe je vaak moeilijke situaties weet om te buigen tot een goed einde. Je constructieve houding en harde werk zullen je zeker brengen waar je wil, veel succes in Vermont en hopelijk tot snel.

Irene, ik heb het altijd fijn gevonden dat ik bij je terecht kon voor een praatje of wetenschappelijke issues. Je overzicht en kennis zijn fantastisch. Op momenten dat het even moeilijk was kon je altijd rust brengen. " Relax Pollie!“ klinkt nog steeds als muziek in de oren. Bedankt voor al je hulp bij verschillende experimenten en vooral je luisterende oor telkens wanneer ik het maar nodig had. Super dat je m'n paranymf wil zijn. Ik wens jou en je man een zalige toekomst!

Er is ook nog meer leven naast het werk, daarom wil ik alle vrienden en vriendinnen bedanken voor het plezier en de gezonde ontspanning. Thanks Mathias voor je interesse en positieve steun de voorbije 22 jaar. Veel succes in Vietnam and let's talk soon captain.

Mijn ganse familie draag ik hoog in het vaandel. Bedankt voor jullie interesse en steun tijdens mijn ganse leven. Vooral mijn zus Julie met haar partner Pieter en kinderen wil ik bedanken; Ondanks het feit dat jullie nooit helemaal volledig op de hoogte waren van wat ik deed, weet ik dat jullie fier waren. Liefste Yvonne je doet me steeds lachen als ik aan je denk, dikke kus! 
Bedankt ook aan de meters en de peters van onze kleintjes, jullie hulp de voorbije jaren was fantastisch. Verder wil ik ook het gezin van Ingrid bedanken. Annemie en Ivo, bedankt voor de nietaflatende hulp met alles. Ik voel me erg thuis bij jullie en kan voor alles bij jullie terecht. Dankjewel. Jullie zijn erg belangrijk voor mij!

Papa, we hebben vele mooie maar ook moeilijke momenten achter de rug. Ik ben blij dat alles beter gaat! Ik kijk uit naar een fijne toekomst samen en ik wens je een goede gezondheid.

Mama, zonder jou stond ik hier niet, c'est simple comme 'bonjour'! Je weet dat ik het fantastisch vind dat ik zoveel waarden van je heb meegekregen en van jongs af aan kon genieten van een enorme vrijheid. Dit heeft me geleerd om op alle niveaus alles uit de kan te halen. Altijd heb je voor me klaar gestaan en gezorgd dat ik en Julie op de eerste plaats kwamen. Ontzettend bedankt, je bent de beste mama ter wereld en daarenboven een super Mammie voor alle kleinkindjes! Ik hoop dat je nog een heerlijke en lange toekomst kan uitbouwen met Jo. Bedankt Jo om zo goed voor m'n mama te zorgen en voor je steun en het nalezen van dit werk. MERCI $\mathrm{x}$.

Bollie, ik ben ontzettend fier op jou en hoe je alles wat je weet te combineren. Wat een leven leiden we toch $(;)$, multi-tasking op zijn toppunt. Knap hoe je steeds constructief kan blijven en mee te zorgen dat we als team alles gesmeerd laten lopen. Het is fantastisch dat je er bent in mijn leven en hoe je altijd klaar staat met woord, daad en de vele kleine attenties. Ik wil je vanzelfsprekend ook prijzen voor al je geduld met me. Bedankt om ons onze parels, Jackie \& Pauline, te schenken. Wat een onwaarschijnlijk parcours hebben we doorzwommen de voorbije 3 jaar. Het was heel zwaar voor ons beide maar tegelijkertijd zou ik het voor geen geld ter wereld willen ruilen. Bedankt voor de prachtige voorbije 12 jaren. Ik zie je enorm graag! Nu kunnen we uitkijken naar het opgroeien van onze spruitjes en het verder plannen van onze toekomst samen. Jackie \& Pauline, jullie hebben onze harten gestolen vanaf de eerste minuut. Liefs, Pollie. 



\section{LIST OF PUBLICATIONS}





\section{LIST OF PUBLICATIONS}

Silica induces NLRP3 inflammasome activation in human lung epithelial cells.

Peeters PM, Perkins TN, Wouters EF, Mossman BT, Reynaert NL.

Part Fibre Toxicol. 2013 Feb 12;10:3. doi: 10.1186/1743-8977-10-3.

Differences in gene expression and cytokine production by crystalline vs. amorphous silica in human lung epithelial cells.

Perkins TN, Shukla A, Peeters PM, Steinbacher JL, Landry CC, Lathrop SA, Steele C, Reynaert NL, Wouters EF, Mossman BT. Part Fibre Toxicol. 2012 Feb 2;9(1):6. doi: 10.1186/1743-8977-9-6.

Silica-Induced NLRP3 Inflammasome Activation In vitro and in Rat Lungs

Peeters PM, Eurlings IM, Perkins TN, Wouters EF, Schins RP, Borm PJ, Drommer W, Reynaert NL, Albrecht C. Part Fibre Toxicol. 2014 Nov 19; 11(1):58.

Indications for distinct pathogenic mechanisms of asbestos and silica through gene expression profiling of the response of lung epithelial cells.

Perkins TN*, Peeters PM*, Shukla A, Arijs I, Dragon J, Wouters EF, Reynaert NL, Mossman BT. Hum Mol Genet. 2014 Oct 27. * = equally contributing 1st authors

Immune homeostasis in epithelial cells: evidence and role of inflammasome signaling reviewed Peeters PM, Wouters EFM, Reynaert NL. Journal of Immunology Research (In Revision)

Pathogenesis and mechanisms of asbestosis and silicosis. Peeters PM, Perkins TN, Wouters EFM, Reynaert NL, Mossman BT. Pathobiology of Human Disease. San Diego: Elsevier; 2014. p. 2654-2664.

Inflammasome activation in human silicosis: Peeters PM, Taeger D, Sanders K, Rohde G, Johnen G, Wouters EFM, Reynaert NL, Pesch B. (In preparation).

Strong upregulation of AIM2 and IFI16 inflammasomes in the mucosa of patients with active inflammatory bowel disease. Wiebe Vanhove*, Paul M. Peeters*, Dominiek Staelens*, Anica Schraenen, Jan Van der Goten, Isabelle Cleynen, Sebastiaan De Schepper, Leentje Van Lommel, Niki L. Reynaert, Frans Schuit, Gert Van Assche, Marc Ferrante, Gert De Hertogh, Emiel F. M. Wouters, Paul Rutgeerts, Séverine Vermeire, Kris Nys \& Ingrid Arijs. (in revision) *=equally contributing $1^{\text {st }}$ authors 



\section{CURRICULUM VITAE}





\section{CURRICULUM VITAE}

Paul Peeters was born on February $1^{\text {st }} 1981$ in Sint-Niklaas, Belgium. He has received his Master degree in Biomedical Sciences at the KU Leuven (BE) in 2005. His Master thesis at the Department of Experimental Cardiac Surgery was entitled: "Contractility characteristics of isolated ventricular cardiomyocytes in animal models of obesity and metabolic syndrome". In 2007 he received his Master degree in Biomedical and Clinical Engineering Techniques with his thesis entitled: "Circadian hearth rhythm variability and autonomic control in astronauts" at the Department of Experimental Cardiology (KU Leuven, BE). After working in a clinical immunological company for about 2 years he joined the Department of Respiratory Medicine at Maastricht University to start his PhD project in December 2009. The first 2 years of his project were performed in the laboratory of Professor Brooke Mossman at the Department of Pathology at the University of Vermont, U.S.A. He returned to Maastricht University to complete his project in December 2014. He was granted with the Long Term Research fellowship 2014-2015 by the European Respiratory Society to be fulfilled at Leibniz Research Institute for Environmental Medicine, Düsseldorf (GE). Here he focuses on "inflammaging" and the inflammasome in the silica model of fibrosis. 



